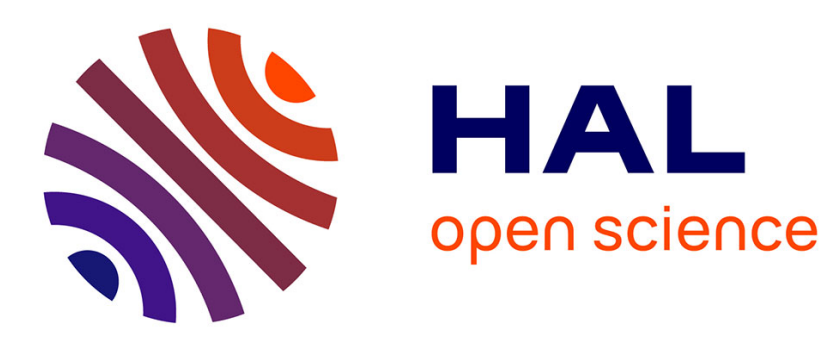

\title{
Statistical analysis of large scale surveys for constraining the Galaxy evolution
}

Andre Machado Murtinheiras Martins

\section{To cite this version:}

Andre Machado Murtinheiras Martins. Statistical analysis of large scale surveys for constraining the Galaxy evolution. Astrophysics [astro-ph]. Université de Franche-Comté, 2014. English. NNT: 2014BESA2026 . tel-01649424

\section{HAL Id: tel-01649424 \\ https://theses.hal.science/tel-01649424}

Submitted on 27 Nov 2017

HAL is a multi-disciplinary open access archive for the deposit and dissemination of scientific research documents, whether they are published or not. The documents may come from teaching and research institutions in France or abroad, or from public or private research centers.
L'archive ouverte pluridisciplinaire HAL, est destinée au dépôt et à la diffusion de documents scientifiques de niveau recherche, publiés ou non, émanant des établissements d'enseignement et de recherche français ou étrangers, des laboratoires publics ou privés. 


\section{'U FC}

Université de Franche-Comté

École Doctorale Carnot-Pasteur

(ED CP n ${ }^{\circ} 554$ )

\section{PhD Thesis}

Specialization: Astrophysics

presented by

\title{
André Machado Murtinheira Martins
}

\section{Statistical analysis of large scale surveys for constraining the Galaxy evolution}

\author{
Directed by Annie Robin \\ Thèse dirigée par Annie Robin \\ soutenue le 9 Décembre 2014
}

Jury :

Dr. Céline Reylé (Président)

Dr. Carine Babusiaux (Rapporteur)

Dr. Francesca Figueras (Rapporteur)

Dr. Olivier Bienaymé (examinateur)

Dr. Annie Robin (Directrice de thèse) 
Front Cover: Astronomy Picture of the Day (2008 January 4) S.Brunier Back cover: ESA/ATG medialab; background: ESO/S. Brunier 


\section{Acknowledgements}

First and foremost, I would like to thank to my $\mathrm{PhD}$ supervisor Dr. Annie Robin, for the knowledge she passed to me, her patience, time and support during these three years of work. It has been an honor to be her Ph.D student. I thank Dr. Francesca Figueras, Dr. Carine Babusiaux, Dr. Olivier Bienaymé and Dr. Céline Reylé which accepted to be the jury of my PhD thesis. I want to thank Céline Reylé for sharing her knowledge and experience. My thanks go also to her husband who is a very good doctor. Thanks go also to the people I encountered at Besançon observatory during my stay in particular Arvind, Ashok, Maria, Esko, Ricardo, Julien and José for productive discussions. The great technical support staff was very during the $\mathrm{PhD}$. I want to thank all of them who helped me with their competences, in particular F. Gazelle, Kevin, Sékou and Bernard Debray. I want to thank to all people in the observatory who helped me integrating in France.

I want to thank the GREAT-ITN that offered me a fellowship and allowed me to be inserted in a large group of professors and $\mathrm{PhD}$ students in this scientific domain. Big thanks to the group and in particular to Sergi, Carmen, Lisa and Cheng for sharing their knowlodge and skills with me. Big thanks to all my former teachers, from Portugal, in particular Dr. João, Dr. Paulo and Dr. Rui for my early formation in this field. A big thanks to Dr. Daniele Galli with whom I learned so much in Arcetri.

A big thanks to all my family for their support and love. In particular my mom, dad, grandmother and sister who have been always at my side. A big thanks to my closest friends in Portugal in particular Antonio Marçal, Antonio, Nuno, Henrique and João. You have always be with me. I want to thanks my closest friends, in the university, in particular Arvind, Ashok, Alia, Leila, Khaoula, Timothée, Mohamad, Alexis, Gaël, Eric Michoulier, Cory and Batoul with whom I shared special moments. Thanks all my friends in particular Yesica, Claudia, Elisa, Ekaterina, Marina, Nicolas and Gustavo with whom I shared both happiness and sadness in my first year in France. I want to thank my friends from Doubs You Play in particular Eric, Jin, Aymeric, Olivier, Hung and Nicolas. I also want to thank Christian and Annick who always had friendly words to tell me.

I want to dedicate this work to all people who were present in my life but in special I dedicate it to the person who has been always in my thoughts and more Ana Maria Vaz Rodrigues. 


\section{Abstract}

The formation and evolution of the thick disc of the Milky Way remain controversial. We made use of a population synthesis model of the Galaxy, the Besançon Galaxy Model (Robin et al. 2003), which can be used for data interpretation, study the Galactic structure and test different scenarios of Galaxy formation and evolution. We examined these questions by studying the shape and the metallicity distribution of the thin and thick disc using the population synthesis approach. We imposed on simulations observational errors and biases to make them directly comparable to observations. We corrected magnitudes and colors of stars, from the simulation, using an extinction model. The available extinction models do not always reproduce the exact quantity of extinction along the line of sight. A code to correct the distribution of extinction in distance along these lines have been developed and the corrected extinctions have been applied on model simulations. We studied the shape of the thin disc using photometric data at low latitudes from the SDSS-SEGUE survey. We compared qualitatively and quantitatively observations and simulations and try to constrain the Initial Mass Function. Using the spectroscopic survey SEGUE we selected Main Sequence Turnoff (MSTO) stars (Cheng et al. 2012b) and K giants to study the metallicity distribution of the thin and thick discs. We computed a distance for each star from the relation between effective temperatures and absolute magnitudes for the observed and simulated catalogs. These two catalogues have the same biases in distances, therefore are comparable. We developed a tool based on a MCMC-ABC method to determine the metallicity distribution and study the correlations between the fitted parameters. We confirmed a radial metallicity gradient of $-0.079 \pm 0.015 \mathrm{dex} \mathrm{kpc}^{-1}$ for the thin disc. We obtained a solar neighborhood metallicity of the thick disc of $-0.47 \pm 0.03$ dex similar to previous studies and the thick disc shows no gradient but the data are compatible with an inner positive gradient followed by a outer negative one. Furthermore, we have applied the developed tools to the Gaia-ESO spectroscopic survey and computed the metallicity distribution of $\mathrm{F} / \mathrm{G} / \mathrm{K}$ stars in the thin and thick disc assuming a two epoch formation for the thick disc of the Milky Way. We obtained a local metallicity in the thick disc of $-0.23 \pm 0.04$ dex slightly higher than the one obtained with SEGUE but in agreement with Adibekyan et al. (2013) and a radial metallicity gradient for the thick disc in agreement with our previous analysis of SEGUE data and the literature. The local metallicity is in fair agreement with literature at the $3 \sigma$ level but because the GES data is an internal release under testing further analysis with more data and better calibrations have to be done. The existence of a flat gradient in the thick disc can be a consequence of an early formation from a highly turbulent homogeneous well mixed gas, unless it has suffered heavy radial mixing later on.

keywords: Galaxy: structure - Galaxy: evolution - Galaxy: formation - Galaxy: disk Galaxy: stellar content - Astronomical data bases: Surveys 


\section{Résumé}

La formation et l'évolution du disque épais de la Voie Lactée restent controversées. Nous avons utilisé un modèle de synthèse de la population de la Galaxie, le Modèle de la Galaxie de Besançon (Robin et al. 2003), qui peut être utilisé pour l'interprétation des données, étudier la structure galactique et tester différents scénario de formation et évolution Galactique. Nous avons examiné ces questions en étudiant la forme et la distribution de métallicité du disque mince et du disque épais en utilisant l'approche de synthèse de la population. Nous avons imposé sur des simulations les erreurs d'observation et les biais afin de les rendre directement comparables aux observations. Nous avons corrigé les magnitudes et les couleurs des étoiles de la simulation, en utilisant un modèle d'extinction. Les modèles d'extinction disponibles ne reproduisent pas toujours la quantité exacte d'extinction le long de la ligne de visée. Un programme a été développé pour corriger la distribution de l'extinction en fonction de la distance le long de ces lignes. Les extinctions correctes ont ensuite été appliquées sur les simulations du modèle. Nous avons étudié la forme du disque mince en utilisant des données photométriques aux basses latitudes du sondage SDSS-SEGUE. Nous avons comparé qualitativement et quantitativement les observations et les simulations et nous avons essayé de contraindre la fonction de masse initiale. En utilisant la spectroscopie du relevé SEGUE, nous avons sélectionné les étoiles du turn-off de la séquence principale (MSTO) (Cheng et al. 2012b) et des géantes K pour étudier la distribution de métallicité du disque mince et du disque épais. Nous avons calculé une estimation de distance pour chaque étoile à partir de la relation entre les températures effectives et magnitudes absolues pour les catalogues observés et simulés. Ces deux catalogues ont les mêmes biais sur les distances, elles sont donc comparables.

Nous avons développé un outil basé sur une méthode MCMC-ABC pour déterminer la distribution de la métallicité et étudier les corrélations entre les paramètres ajustés. Nous avons confirmé la présence d'un gradient de métallicité radiale de $-0.079 \pm 0.015 \mathrm{dex} \mathrm{kpc}^{-1}$ pour le disque mince. Nous avons obtenu une métallicité du disque épais au voisinage solaire de $-0.47 \pm 0.03$ dex, compatible avec les résultats obtenus par les études précédentes. De plus, le disque épais ne montre pas de gradient, mais les données sont compatibles avec un gradient positif intérieur suivi d'un négatif extérieur. Nous avons ensuite appliqué les outils développés au relevé spectroscopique Gaia-ESO et calculé la distribution de métallicité des étoiles $\mathrm{F} / \mathrm{G} / \mathrm{K}$ dans le disque mince et épais en supposant une formation en deux époques du disque épais de la Voie Lactée. Nous avons obtenu une métallicité locale dans le disque épais de $-0.23 \pm 0.04$ dex légèrement plus élevée que celle obtenue avec SEGUE mais en accord avec Adibekyan et al. (2013) et un gradient de métallicité radiale du disque épais en accord avec notre analyse précédente des données de SEGUE et la littérature. La métallicité locale est en accord avec la littérature au niveau de $3 \sigma$ mais parce que les données GES sont préliminaires, une analyse plus approfondie avec plus de données et de meilleurs calibrations doit être faite. L'existence d'un 
gradient plat dans le disque épais peut être une conséquence d'une formation à partir d'un gaz turbulent et bien homogène, ou bien un fort mélange radial a brassé après coup les étoiles.

Mots clés: Galaxy: structure - Galaxy: evolution - Galaxy: formation - Galaxy: disk Galaxy: stellar content - Astronomical data bases: Surveys 


\section{Contents}

1. Motivation 1

2. Introduction 3

2.1. Astronomy in the antiquity ................... 3

2.2. Galaxy formation in a $\Lambda$ cold dark matter scenario . . . . . . . . . . 5

2.3. Structure of the Milky Way . . . . . . . . . . . . . . . . . . . 7

2.4. Galactic populations . . . . . . . . . . . . . . . 10

2.5. Disc of the Milky Way . . . . . . . . . . . . . . . . . 11

2.5.1. Thin disc . . . . . . . . . . . . . . . . 12

2.5.2. Thick disc . . . . . . . . . . . . . . . . . 13

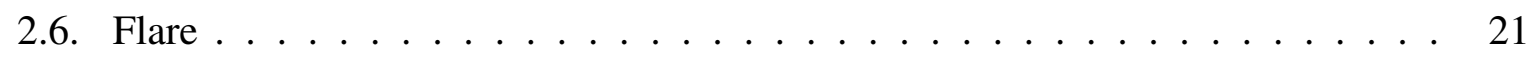

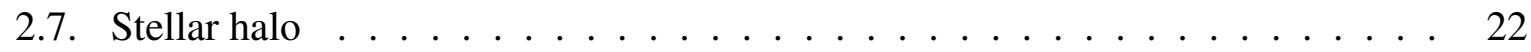

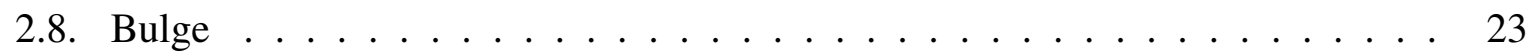

3. The Besançon Galaxy Model 25

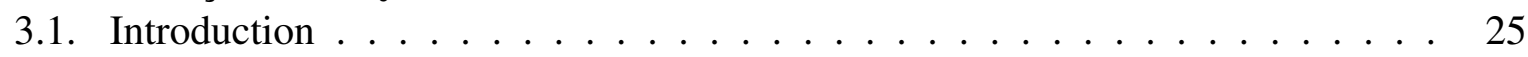

3.2. The overall structure . . . . . . . . . . . . . . 26

3.2.1. The luminosity function and Hess diagram . . . . . . . . . . 26

3.2.2. Density laws . . . . . . . . . . . . . . . . 28

3.2.3. Model kinematics . . . . . . . . . . . . . . . . . . . . . . . . 30

3.2.4. The dynamical self-consistency . . . . . . . . . . . . 31

3.3. The metallicity distribution . . . . . . . . . . . . . . . 31

3.4. A revised model for the thin disc . . . . . . . . . . . . . . . . 32

3.4.1. Atmosphere models ... . . . . . . . . . . . . . . . 33

3.4.2. Evolutionary tracks . . . . . . . . . . . . . . 33

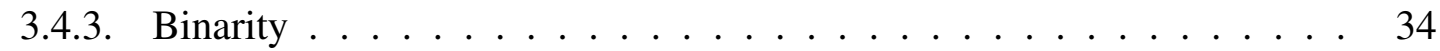

3.4.4. Age-metallicity relation . . . . . . . . . . . . . 34

3.4.5. Dynamical mass . . . . . . . . . . . . . . . . . . . 35

3.5. A new thick disc in the BGM . . . . . . . . . . . . . 36

$\begin{array}{ll}\text { 4. Surveys } & 37\end{array}$

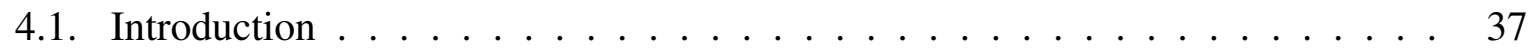

4.2. Geneva-Copenhagen survey . . . . . . . . . . . . . . 37

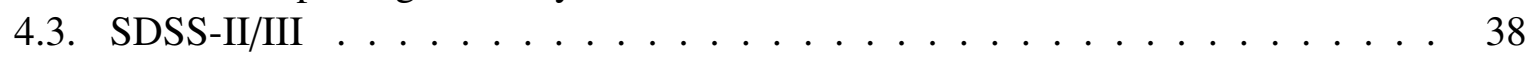

4.3.1. SEGUE/SEGUE2 . . . . . . . . . . . . . . . . . . 38 


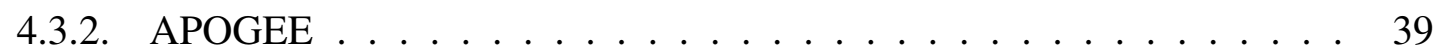

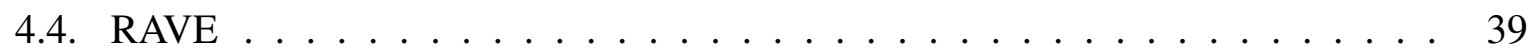

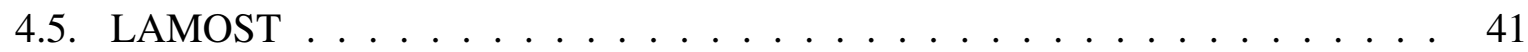

4.6. Gaia .............................. 41

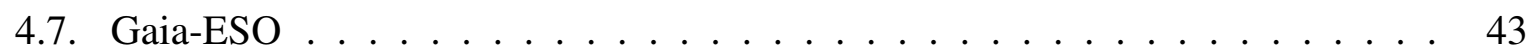

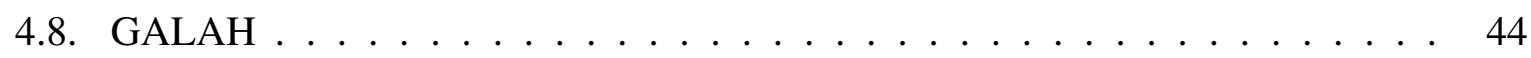

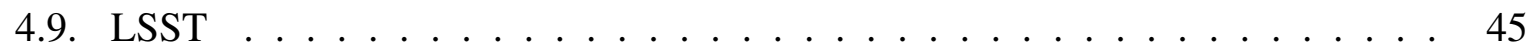

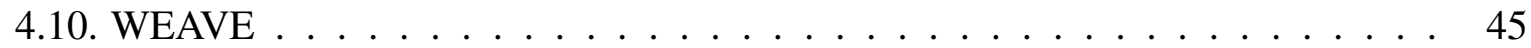

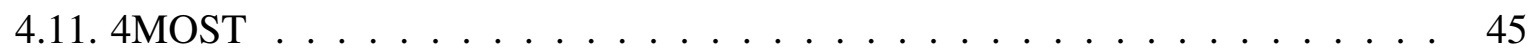

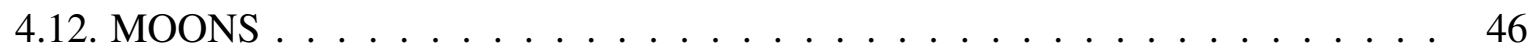

5. Photometric and spectroscopic sample 47

5.1. Selecting the photometric sample from SEGUE . . . . . . . . . . . . . . . 47

5.2. Selecting the spectroscopic sample from SEGUE . . . . . . . . . . . . . . . 49

6. Simulations $\quad 51$

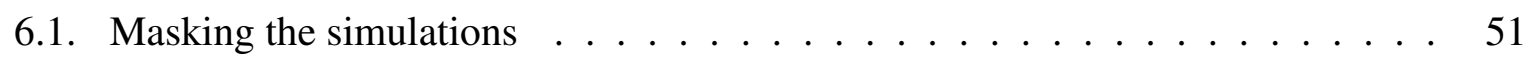

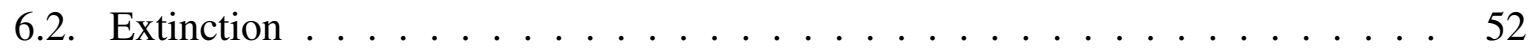

6.3. The $\mathrm{S} / \mathrm{N}$, proper motions and spectral parameter errors $\ldots \ldots \ldots \ldots$

6.4. Selection Sample . . . . . . . . . . . . . . . . . . . 73

6.4.1. Main Sequence turnoff selection . . . . . . . . . . . . . . . . . . 73

6.4.2. K giants selection . . . . . . . . . . . . . . . . . . 74

7. Photometric results $\quad \mathbf{7 5}$

7.1. Comparison between observations and simulations _ . . . . . . . . . 75

7.1.1. Fields 2534 and $2536 \ldots \ldots \ldots$. . . . . . . . . . . 75

7.1.2. Fields 2537 and $2538 \ldots \ldots \ldots$. . . . . . . . . . . . . . . . . . . . . . . . . . . . .

7.1.3. Fields 2554,2555 and $2556 \ldots \ldots \ldots$. . . . . . . . . . 90

7.1.4. Fields 2668, 2678 and $2681 \ldots \ldots \ldots$. . . . . . . . . . . 104

7.1.5. Proper motions . . . . . . . . . . . . . . . . . 117

7.2. Constraining the Initial Mass Function . . . . . . . . . . . . . . . . . . 119

7.2.1. Method . . . . . . . . . . . . . . . . 119

7.2.2. Results . . . . . . . . . . . . . . . . . 120

7.3. Comparison with a new version of the Besançon galaxy model . . . . . . . . 121

8. Spectroscopic results from the SEGUE low latitude data 125

8.1. Main Sequence Turn off stars . . . . . . . . . . . . . . . . . 125

8.1.1. Comparison between observations and simulations . . . . . . . . . 125

8.1.2. Preliminary comparison with the standard model . . . . . . . . . . 125

8.1.3. Metallicity variation with longitudes and latitudes . . . . . . . . . . . . . 128

8.1.4. Distances . . . . . . . . . . . . . . . . . . . . . 129

8.1.5. Fitting method . . . . . . . . . . . . . . 131

8.1.6. Case 1 . . . . . . . . . . . . . . . . . 133

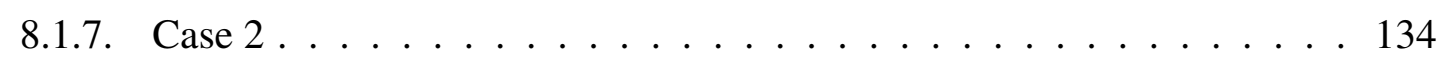

8.1.8. Case $3 \ldots \ldots \ldots \ldots \ldots \ldots$. . . . . . . . . . . . . . . . . . . . . . 
8.1.9. Revised model . . . . . . . . . . . . . . . . . . . . . . 138

8.1.10. Fitting two slopes in the thick disc . . . . . . . . . . . . . . 141

8.1.11. The age of the thick disc . . . . . . . . . . . . . . . 143

8.2. The metallicity distribution in the model B revised version . . . . . . . . . . 143

8.3. K giants sample . . . . . . . . . . . . . . . . . . . . . . . . . 144

8.4. Comparison with previous works . . . . . . . . . . . . . . . . 144

8.4.1. Thin disc . . . . . . . . . . . . . . . . . . . 144

8.4.2. Thick disc . . . . . . . . . . . . . . . . . 146

9. Spectroscopic results from the GES data 149

9.1. Photometric errors . . . . . . . . . . . . . . . . . . . . . . 149

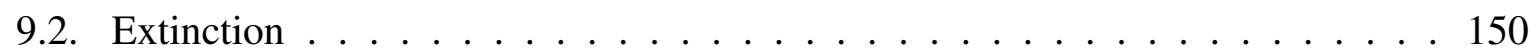

9.3. Spectroscopic errors . . . . . . . . . . . . . . . . . . . 157

9.4. The selection sample . . . . . . . . . . . . . . . . . . . 157

9.5. Distances . . . . . . . . . . . . . . . . . . . 161

9.6. The Metallicity Distribution . . . . . . . . . . . . . . . . . 166

9.6.1. The observed metallicity distribution . . . . . . . . . . . . 166

9.6.2. Preliminary comparison with the BGM . . . . . . . . . . . . . . 168

9.6.3. ABC/MCMC analysis for the GES sample . . . . . . . . . . . . . . . . 174

9.7. Discussion . . . . . . . . . . . . . . . . 176

9.7.1. The thin disc . . . . . . . . . . . . . . 176

9.7.2. The thick disc . . . . . . . . . . . . . . . . . 178

10. Conclusions and perspectives 179

10.1. The photometric sample . . . . . . . . . . . . . . . . . . 179

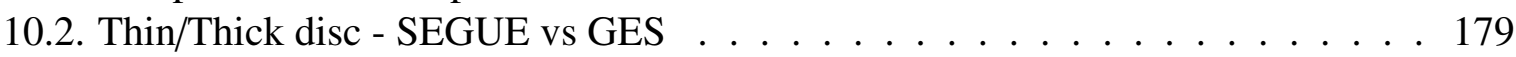

10.3. If the thick disc has no radial gradient? . . . . . . . . . . . . . . . . . 182

10.3.1. If the thick disc has a positive/negative gradient? . . . . . . . . . 183

10.3.2. Perspectives . . . . . . . . . . . . . . . . 183

$\begin{array}{ll}\text { Publications } & 185\end{array}$

$\begin{array}{ll}\text { Bibliographie } & 185\end{array}$ 


\section{List of Figures}

2.1. Herschel map of the Milky Way. The darker black point near the center of the map is the Sun position. Source: On the Construction of the Heavens. By William Herschel, Esq. F. R. S. Philosophical Transactions of the Royal Society of London, Vol. 75. (1785), pp. 213-266. . . . . . . . . . . . . . . . 5

2.2. Kapteyn's map of the Galaxy. Source: Kapteyn (1922) . . . . . . . . . . . . .

2.3. An illustration of the concept of baryon acoustic oscillations, which are imprinted in the early universe and can still be seen today in Galaxy surveys like the Baryon Oscillation Spectroscopic Survey (BOSS; Schlegel et al. 2009). Image Credit: Chris Blake and Sam Moorfield in https://www.sdss3.org/surveys/boss.php

2.4. The Milky Way edge-on view showing the different components of the Milky Way with the Sun position indicated. Image Credit: R. Buser. Hurt . . . . . . . 8

2.5. The Milky Way face-on view with the Sun position indicated. Artist's impression of the Milky Way. Image Credit: NASA/JPL-Caltech/ESO/R. . . . . . . .

2.6. The observed and predicted rotation curve of the Galaxy M33. Image credit: University of Sheffield. . . . . . . . . . . . . . . . . . . .

2.7. The predicted rotation curve from the self-consistent model of the Milky Way Robin et al. (2003). The contributions from each component of the Milky Way is also visible. The observations are from Caldwell \& Ostriker (1981) . . . . .

2.8. A scheme showing the orbits of population I and II.Image credit: Nick Strobel at www.astronomynotes.com.

2.9. Schematic representation of a disc structure. Artist's impression of the Milky Way. Image Credit: Amanda Smith, IoA graphics officer . . . . . . . . . . . .

2.10. The NGC 4762 Galaxy, in different filters (B and V filters), showing a thin and a thick disc (Tsikoudi 1980). The image is taken from http://arxiv.org/ps/astroph/0208106v1 figure 3 in Freeman \& Bland-Hawthorn (2002) . . . . . . . . .

2.11. Warp of the spiral Galaxy ESO 510-13. Image Credit: NASA's Hubble Space Telescope . . . . . . . . . . . . . . . . . . .

2.12. The dust warp, in galactic coordinates, as traced by extinction from figure 8 of Marshall et al. (2006). Each panel describes the extinction at $1 \mathrm{kpc}$ intervals from the nearest (top panel) to the further (bottom panel) distance. The black line indicates the position of the mid plane as given by the stellar warp formula in Robin et al. (2003). . . . . . . . . . . . . . . . . . . . . . . . .

2.13. Schematic representation of the galactic structure. The flare is represented by the discontinuous line representing the thick disc which increases scale height as a function of the galactocentric radius. Image Credit: Gaia-ESO Survey . . . 
2.14. Figure from Minniti \& Zoccali (2008). The velocity dispersion and mean velocity as a function of the galactic longitude in the bulge component. Blue squares are K giants measured by Minniti (1996) and red crosses are M-giants measured by Rich et al. (2007) and which were corrected for the solar motion around the Galaxy. . . . . . . . . . . . . . . . . . . .

3.1. Figure 1 of Robin \& Crézé (1986). Flowchart of the algorithm.

3.2. Scheme of the algorithm to compute the dynamical self-consistency. Ingredients for the equations are shown in italic, equations are shown in bold and results from equations are underlined. . . . . . . . . . . .

4.1. The SDSS sky coverage in the southern and northern Galactic cap. Image credit: http://www.sdss.org/sdss-surveys/ . . . . . . . . . . . . . . .

4.2. The SEGUE in blue and SEGUE-2 in red sky coverage in Galactic coordinates. Image credit: M. Strauss in http://www.sdss3.org/surveys/segue2.php . . . . .

4.3. The APOGEE sky coverage, from DR10, in Galactic coordinates. Image credit: in http://www.sdss3.org/dr10/ . . . . . . . . . . . . . . . .

4.4. The RAVE sky coverage where color are the stellar heliocentric radial velocities. Image credit: Axel Mellinger at http://www.rave-survey.aip.de/rave/pages/project/index.jsp

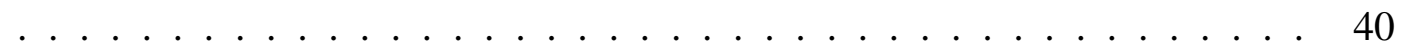

4.5. Simulation of the Gaia Galactic coverage. Image credit: X. Luri \& the DPAC-

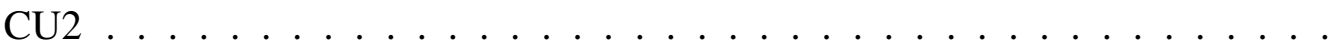

4.6. The Gaia Galactic coverage where the smaller and larger circles indicate the radius at which distances are accurate to $10 \%$ and the tangential velocities accurate to $1 \mathrm{~km} \mathrm{~s}^{-1}$. Image credit: ESA/Lund . . . . . . . . . . . . . .

4.7. The Gaia parallax errors. Top left panel: The G0V stars parallax error distribution without extinction. Top right panel: The G0V stars parallax error distribution with extinction. Bottom left panel: The K5III stars parallax error distribution with extinction and no extinction. Bottom right panel: The Cepheids stars parallax error distribution with extinction and no extinction Image credit: Lennart Lindegren . . . . . . . . . . . . . . . . . .

4.8. Sky coverage of the observed targets in DR1 data release. Color indicates the different target groups. MW: Milky Way fields;CL: Clusters; SD: Standard Image credit: provided by Cambridge Astronomy Survey Unit (CASU) http://casu.ast.cam.ac.uk/gaiaeso/overview . . . . . . . . . .

5.1. Difference between the SSPP temperature estimates and the corrected values as a function of the SSPP temperature estimates for plate $2536(1, \mathrm{~b})=\left(70^{\circ}, 14^{\circ}\right)$. . .

5.2. Difference between the SSPP temperature estimates and the corrected values as a function of the extinction $\mathrm{E}(\mathrm{B}-\mathrm{V})$ for plate $2555(1, \mathrm{~b})=\left(94^{\circ}, 8^{\circ}\right) \ldots \ldots \ldots$

6.1. Distribution in $(1, b)$ of the simulated stars (red points) and observed ones (blue points) for the field 2537. . . . . . . . . . . . . . . . . .

6.2. The green points are the masked simulation and the red points are the full simulation for the field $2537 \ldots \ldots \ldots \ldots$ 
6.3. Map of the Schlegel et al. (1998) extinction in the g band for the field 2536. We can see in this figure regions where the extinction is homogeneous and low and regions where the extinction is higher. . . . . . . . . . . .

6.4. Map of the Schlegel et al. (1998) extinction in the $g$ band for the field 2555 . We can see in this figure regions where the extinction in $\mathrm{g}$ band can reach 8 magnitudes. . . . . . . . . . . . . . . . .

6.5. Map of the Drimmel \& Spergel (2001) extinction model in the g band for the field 2536. . . . . . . . . . . . . . . . . . . . . .

6.6. Color magnitude diagram for the field $2536(1, \mathrm{~b})=\left(70^{\circ}, 14^{\circ}\right)$. Density map along with grey contours are observations. Black contours are simulations.

6.7. Color magnitude diagram for the field $2555(1, b)=\left(94^{\circ}, 8^{\circ}\right)$. Density map along with grey contours are observations. Black contours are simulations. . . . . . . .

6.8. Top panel: Total $\mathrm{A}_{v}$ as a function of the distance from the Sun for field 2534 $(1, b)=\left(50^{\circ}, 14^{\circ}\right)$. Density contours are the total $\mathrm{A}_{v}$ after correction and black contours are before the correction. Bottom panel: Total $\mathrm{A}_{v}$ distribution before (red dashed line) and after correction (solid black line). . . . . . . . . . . .

6.9. Total $\mathrm{A}_{v}$ as a function of the distance from the Sun for field $2536(1, \mathrm{~b})=\left(70^{\circ}, 14^{\circ}\right)$. Density contours are the total $\mathrm{A}_{v}$ after correction and black contours are before the correction. Bottom panel: Total $\mathrm{A}_{v}$ distribution before (red dashed line) and after correction (solid black line). . . . . . . . . . . . . . . . . 57

6.10. Total $\mathrm{A}_{v}$ as a function of the distance from the Sun for field $2537(1, b)=\left(110^{\circ}, 10.5^{\circ}\right)$. Density contours are the total $\mathrm{A}_{v}$ after correction and black contours are before the correction. Bottom panel: Total $\mathrm{A}_{v}$ distribution before (red dashed line) and after correction (solid black line) . . . . . . . . . . . . . . . . .

6.11. Total $\mathrm{A}_{v}$ as a function of the distance from the Sun for field $2538(1, \mathrm{~b})=\left(110^{\circ}, 16^{\circ}\right)$. Density contours are the total $\mathrm{A}_{v}$ after correction and black contours are before the correction. Bottom panel: Total $\mathrm{A}_{v}$ distribution before (red dashed line) and after correction (solid black line). . . . . . . . . . . . . .

6.12. Total $\mathrm{A}_{v}$ as a function of the distance from the Sun for field $2554(1, \mathrm{~b})=\left(94^{\circ}, 14^{\circ}\right)$. Density contours are the total $\mathrm{A}_{v}$ after correction and black contours are before the correction. Bottom panel: Total $\mathrm{A}_{v}$ distribution before (red dashed line) and after correction (solid black line). . . . . . . . . . . . . .

6.13. Total $A_{v}$ as a function of the distance from the Sun for field $2555(1, b)=\left(94^{\circ}, 8^{\circ}\right)$. Density contours are the total $\mathrm{A}_{v}$ after correction and black contours are before the correction. Bottom panel: Total $\mathrm{A}_{v}$ distribution before (red dashed line) and after correction (solid black line). . . . . . . . . . . . . .

6.14. Total $A_{v}$ as a function of the distance from the Sun for field $2556(1, b)=\left(94^{\circ}\right.$,$\left.8^{\circ}\right)$. Density contours are the total $\mathrm{A}_{v}$ after correction and black contours are before the correction. Bottom panel: Total $\mathrm{A}_{v}$ distribution before (red dashed line) and after correction (solid black line). . . . . . . . . . . . . . .

6.15. Total $\mathrm{A}_{v}$ as a function of the distance from the Sun for field $2668(1, \mathrm{~b})=\left(187^{\circ}\right.$,$12^{\circ}$ ). Density contours are the total $\mathrm{A}_{v}$ after correction and black contours are before the correction. Bottom panel: Total $\mathrm{A}_{v}$ distribution before (red dashed line) and after correction (solid black line) . . . . . . . . . . . . 
6.16. Total $\mathrm{A}_{v}$ as a function of the distance from the Sun for field $2678(1, \mathrm{~b})=\left(187^{\circ}\right.$,$8^{\circ}$ ). Density contours are the total $\mathrm{A}_{v}$ after correction and black contours are before the correction. Bottom panel: Total $\mathrm{A}_{v}$ distribution before (red dashed line) and after correction (solid black line). . . . . . . . . . . . . . .

6.17. Total $\mathrm{A}_{v}$ as a function of the distance from the Sun for field $2681(1, b)=\left(178^{\circ},-\right.$ $15^{\circ}$ ). Density contours are the total $\mathrm{A}_{v}$ after correction and black contours are before the correction. Bottom panel: Total $\mathrm{A}_{v}$ distribution before (red dashed line) and after correction (solid black line). . . . . . . . . . . . . . .

6.18. Color-magnitude diagram for region one of field $2555(1, b)=\left(94^{\circ}, 8^{\circ}\right)$. Density map along with grey contours are observations. Black contours are simulations.

6.19. S/N as a function of the $\mathrm{r}$ magnitude for the plate 2668. Black points are observations and red points are simulations after applying the fit. . . . . . . . .

6.20. Parameter errors as a function of the $\mathrm{S} / \mathrm{N}$ for plate 2699. Top left panel shows the metallicity error, bottom left: effective temperature, top right: gravity, bottom right: radial velocity. . . . . . . . . . . . . . . .

7.1. Magnitude distribution for each individual region of the field 2536. The black histograms are observations and the red histograms are simulations. . . . . . .

7.2. Color distribution for each individual region of the field 2536. The black histograms are observations and the red histograms are simulations. . . . . . . . .

7.3. Magnitude, color (top panels) and proper motion distributions (middle and bottom panels) for field 2536. Black histograms are observations and the red histograms are simulations.

7.4. Simulated magnitude, color (top panels) and proper motion distributions (bottom panels) separated by populations for field 2536. Thin disc stars: Black solid line; Thick disc stars: Red dotted line; Halo stars: Blue dashed line.

7.5. Magnitude distribution for each individual region of the field 2537. The black histograms are observations and the red histograms are simulations.

7.6. Color distributions for each individual region of the field 2537. The black histograms are observations and the red histograms are simulations.

7.7. Magnitude, color (top panels) and proper motion distributions (middle and bottom panels) for field 2537. Black histograms are observations and the red histograms are simulations.

7.8. Magnitude, color (top panels) and proper motion distributions (bottom panels) separated by populations for field 2537. Thin disc stars: Black solid line; Thick disc stars: Red dotted line; Halo stars: Blue dashed line.

7.9. Magnitude distribution for each individual region of the field 2537. The black histograms are observations and the red histograms are simulations. . . . . . .

7.10. Color distributions for each individual region of the field 2537. The black histograms are observations and the red histograms are simulations.

7.11. Magnitude, color (top panels) and proper motion distributions (middle and bottom panels) for field 2538. Black histograms are observations and the red histograms are simulations. 
7.12. Magnitude, color (top panels) and proper motion distributions (bottom panels) separated by populations for field 2538. Thin disc stars: Black solid line; Thick disc stars: Red dotted line; Halo stars: Blue dashed line. . . . . . . . . . . . .

7.13. Magnitude distribution for each individual region of the field 2554. The black histograms are observations and the red histograms are simulations. . . . . . .

7.14. Magnitude distribution for each individual region of the field 2555. The black histograms are observations and the red histograms are simulations. . . . . . .

7.15. Magnitude distribution for each individual region of the field 2556. The black histograms are observations and the red histograms are simulations. . . . . . .

7.16. Color distribution for each individual region of the field 2554. The black histograms are observations and the red histograms are simulations. . . . . . . .

7.17. Color distribution for each individual region of the field 2555. The black histograms are observations and the red histograms are simulations. . . . . . . .

7.18. Color distribution for each individual region of the field 2556. The black histograms are observations and the red histograms are simulations. . . . . . . .

7.19. Magnitude, color (top panels) and proper motion distributions (middle and bottom panels) for field 2554. Black histograms are observations and the red histograms are simulations. . . . . . . . . . . . . . .

7.20. Magnitude, color (top panels) and proper motion distributions (bottom panels) separated by populations for field 2554. Thin disc stars: Black solid line; Thick disc stars: Red dotted line; Halo stars: Blue dashed line.

7.21. Magnitude, color (top panels) and proper motion distributions (middle and bottom panels) for field 2555. Black histograms are observations and the red histograms are simulations.

7.22. Magnitude, color (top panels) and proper motion distributions (bottom panels) separated by populations for field 2555. Thin disc stars: Black solid line; Thick disc stars: Red dotted line; Halo stars: Blue dashed line.

7.23. Magnitude, color (top panels) and proper motion distributions (middle and bottom panels) for field 2556. Black histograms are observations and the red histograms are simulations.

7.24. Magnitude, color (top panels) and proper motion distributions (bottom panels) separated by populations for field 2556. Thin disc stars: Black solid line; Thick disc stars: Red dotted line; Halo stars: Blue dashed line.

7.25. Magnitude distribution for each individual region of the field 2668. The black histograms are observations and the red histograms are simulations. . . . . . .

7.26. Magnitude distribution for each individual region of the field 2678. The black histograms are observations and the red histograms are simulations. . . . . . 106

7.27. Magnitude distribution for each individual region of the field 2681. The black histograms are observations and the red histograms are simulations. . . . . . .

7.28. Color distribution for each individual region of the field 2668. The black histograms are observations and the red histograms are simulations. . . . . . . . 108

7.29. Color distribution for each individual region of the field 2678. The black histograms are observations and the red histograms are simulations. . . . . . . . . 109

7.30. Color distribution for each individual region of the field 2681. The black histograms are observations and the red histograms are simulations. . . . . . . . 110 
7.31. Magnitude, color (top panels) and proper motion distributions (middle and bottom panels) for field 2668. Black histograms are observations and the red histograms are simulations.

7.32. Magnitude, color (top panels) and proper motion distributions (bottom panels) separated by populations for field 2668. Thin disc stars: Black solid line; Thick disc stars: Red dotted line; Halo stars: Blue dashed line.

7.33. Magnitude, color (top panels) and proper motion distributions (middle and bottom panels) for field 2678. Black histograms are observations and the red histograms are simulations . . . . . . . . . . . . .

7.34. Magnitude, color (top panels) and proper motion distributions (bottom panels) separated by populations for field 2678. Thin disc stars: Black solid line; Thick disc stars: Red dotted line; Halo stars: Blue dashed line.

7.35. Magnitude, color (top panels) and proper motion distributions (middle and bottom panels) for field 2681. Black histograms are observations and the red histograms are simulations.

7.36. Magnitude, color (top panels) and proper motion distributions (bottom panels) separated by populations for field 2681. Thin disc stars: Black solid line; Thick disc stars: Red dotted line; Halo stars: Blue dashed line.

7.37. Proper motion (in mas $y r^{-1}$ ) measures of central tendency (Mean and Mode) and of dispersion (Standard deviation) as a function of the galactic coordinates. black filled circles: mean of the observations; black open circles: mode of the observations; red filled triangles: mean of the simulations; red open triangles: mode of the simulations. Top panel: 1 and $b$ components of the proper motion as a function of the galactic longitude. Bottom panel: 1 and $b$ components of the proper motion as a function of the galactic latitude. . . . . . . . . . . 118

7.38. Mass distribution for the fields used to fit the IMF as given by the standard model.120

7.39. $\chi^{2}$ as a function of the IMF slopes. . . . . . . . . . . . . . . 121

7.40. $\chi^{2}$ for the magnitude distribution as a function of galactic longitude. The color coding is galactic latitude. Circles are the original simulation and squares are model $\mathrm{B}$ of the revised version of the BGM.

7.41. $\chi^{2}$ for the color distribution as a function of galactic longitude. The color coding is galactic latitude. Circles are the original simulation and squares are model B of the revised version of the BGM.

7.42. $\chi^{2}$ for the color magnitude distribution as a function of galactic longitude. The color coding is galactic latitude. Circles are the original simulation and squares are model B of the revised version of the BGM.

8.1. Distance from the plane as a function of the galactocentric distance for the MSTO stars sample as given by the simulations. . . . . . . . . . . . . 126

8.2. Comparison of the spectrocopic observations and simulations for the bright plate 2537, $l=110^{\circ}$ and $b=10.5^{\circ}$. Black points and lines are observations. Simulations are in red.

8.3. Comparison of the spectrocopic observations and simulations for the faint plate $2545, l=110^{\circ}$ and $b=10.5^{\circ}$. Black points and lines are observations. Simulations are in red. 
8.4. Mode of metallicities at different longitudes for the data and the standard model. left panel are the bright plates and right panel are the faint plates. The observations are in black and the simulations in red. The standard deviation is represented by the small bars. Squares are latitudes higher or equal to $14^{\circ}$; Circles are latitudes between $8^{\circ}$ and $10.5^{\circ}$; Triangles are latitudes equal to $-8^{\circ}$; Diamonds correspond to latitudes $-12^{\circ}$ and $-15^{\circ}$.

8.5. Relation between effective temperature and absolute magnitude for the different metallicity ranges. Top panel: $[\mathrm{Fe} / \mathrm{H}] \leq-0.5$. Middle panel: $-0.5<[\mathrm{Fe} / \mathrm{H}]<$ 0.0. Bottom panel: $[\mathrm{Fe} / \mathrm{H}] \geq 0.0$. Black points are simulations and the red line is the fit.

8.6. Difference between the new computed distance and the distance given by the model as a function of the distance given by the model. The red line is an horizontal line that crosses the $\mathrm{Y}$ axis at zero.

8.7. Difference between distance estimate and the distance given by the model as a function of the $\log$ (distance) given by the model. Density map and grey contours refer to our method and black contours to Ivezić et al. (2008) method.

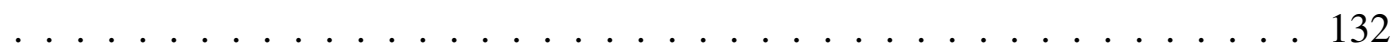

8.8. Correlations between parameters of the thick disc and old thin disc for case 1. . 135

8.9. Correlations between parameters for the thick disc and old thin disc fitting for

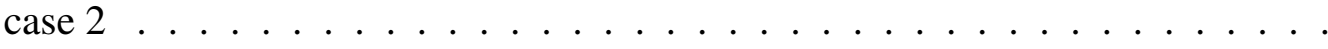

8.10. Mode of metallicities at different longitudes for the revised model (case 1). Left panel are the bright plates and right panel are the faint plates. The observations are in black and the simulations in red. The standard deviation is represented by the small bars. Squares are latitudes higher or equal to $14^{\circ}$; Circles are latitudes between $8^{\circ}$ and $10.5^{\circ}$; Triangles are latitudes equal to $-8^{\circ}$; Diamonds are latitudes lower than $-8^{\circ}$.

8.11. Effective temperature distributions for the original model (top panel) and revised model (bottom plot) for plate 2538. Black lines are observations and red lines simulations.

8.12. Top panel: total extinction applied to the simulations as a function of the distance to the Sun. Density plot refers to the original simulation and black contours to the revised version. Bottom panel: Total extinction distribution applied to the original model (black lines) and to the revised model (red lines).

8.13. Comparison of the spectrocopic observations and simulations for the bright plate 2536. . . . . . . . . . . . . . . . . .

8.14. Comparison of the spectrocopic observations and simulations for the faint plate 2544.

9.1. Reddening from SFD maps and color distributions from GES data (black lines) and simulations (red lines) for field 074500423000 (top panel) and field 154224 441200 (bottom panel).

9.2. Reddening from SFD maps and color distributions from GES data (black lines) and simulations (red lines) for field 155400410000 (top panel) and field 160312 455359 (bottom panel). 
9.3. Reddening from SFD maps and color distributions from GES data (black lines) and simulations (red lines) for field 170024051200 (top panel) and field 173359 430000 (bottom panel). . . . . . . . . . . . . . . . 156

9.4. Aitoff projection for the iDR2 fields selected for this analysis. . . . . . . . . . 161

9.5. Spatial distribution for the $\mathrm{F} / \mathrm{G} / \mathrm{K}$ stars sample from $\mathrm{GES}$ as given by the simulations. . . . . . . . . . . . . . . . . . . . 161

9.6. Absolute magnitude as a function of the effective temperature for dwarf stars in the three different metallicity ranges. From top to bottom the metallicity ranges are $[\mathrm{Fe} / \mathrm{H}]<-0.5,-0.5 \leq[\mathrm{Fe} / \mathrm{H}] \leq 0.0$ and $[\mathrm{Fe} / \mathrm{H}]>0.0$ respectively. . . .

9.7. Absolute magnitude as a function of the effective temperature for giants stars in the three different metallicity ranges. From top to bottom the metallicity ranges are $[\mathrm{Fe} / \mathrm{H}]<-0.5,-0.5 \leq[\mathrm{Fe} / \mathrm{H}] \leq 0.0$ and $[\mathrm{Fe} / \mathrm{H}]>0.0$ respectively. . . .

9.8. Absolute magnitude as a function of the $\log g$ for subgiants stars in the two different metallicity ranges. From top to bottom the metallicity ranges are $[\mathrm{Fe} / \mathrm{H}] \leq-0.5$ and $[\mathrm{Fe} / \mathrm{H}]>-0.5$ respectively. . . . . . . . . . .

9.9. Difference between distance estimate and the distance given by the model as a function of the $\log$ (distance) (natural logarithm) given by the model. Density map and grey contours refer to our method. . . . . . . . . . . . . .

9.10. Distribution of the differences between distance estimate and the distance given by the model. . . . . . . . . . . . . . . . .

9.11. Metallicity distribution of the total GES sample from observations (grey lines) and as given by simulations (Green lines). The different components as given by the model are: black lines: Thin disc; red lines: Young thick disc; blue lines: Old thick disc; orange lines: Halo. . . . . . . . . . . . . . . . . .

9.12. Comparison of the spectrocopic observations and simulations for a field at $1=229^{\circ}$ and $b=18^{\circ}$. Left panels: Black lines are observations and simulations are in red. The Poisson noise is represented by the small vertical bars. Right panels: Black lines are thin disc; red lines are young thick disc; blue lines are old thick disc; orange lines are halo. . . . . . . . . . . . . . . .

9.13. Comparison of the spectrocopic observations and simulations for a field at $1=97^{\circ}$ and $b=-61^{\circ}$. Left panels: Black lines are observations and simulations are in red. The Poisson noise is represented by the small vertical bars. Right panels: Black lines are thin disc; red lines are young thick disc; blue lines are old thick disc; orange lines are halo. . . . . . . . . . . . . . . .

9.14. Effective temperature as a function of the $\mathrm{J}-\mathrm{K}$ color for a field at $1=97^{\circ}$ and $\mathrm{b}=-61^{\circ}$. Black points represent observations and red points simulations.

9.15. Distribution in $(1, b)$ for all fields in GES sample. The color code represents the difference between the mean observation and mean simulation for each field.

9.16. Distribution in $(1, b)$ for all fields in GES sample. The color code represents the difference between mode of the observed distribution in metallicity and the mode of the simulated distribution. . . . . . . . . . . . . .

9.17. Correlations between parameters for the young thick disc and old thin disc fitting for the GES sample analysis. 
10.1. Galactocentric radius distribution for the $\mathrm{F} / \mathrm{G} / \mathrm{K}$ stars sample from GES (red line) and MSTO from SEGUE (blue line) as given by the simulations. . . . . . 180

10.2. Distance from the plane distribution for the $\mathrm{F} / \mathrm{G} / \mathrm{K}$ stars sample from GES (red line) and MSTO from SEGUE (blue line) as given by the simulations. . . . . . 180

10.3. Metallicity as a function of the galactocentric radius for the case 1 SEGUE results (red lines) and GES SN metallicity and gradient results for the old thin disc. The continuous lines are the best values obtained and the dashed lines are the combination of the different results taking into account the maximum and minimum values for the errors. . . . . . . . . . . . . . . . . . . 181

10.4. Metallicity as a function of the galactocentric radius for the SEGUE results (red lines) and GES SN metallicity and gradient results for the thick disc. The continuous lines are the best values obtained and the dashed lines are the combination of the different results taking into account the maximum and minimum values for the errors 


\section{List of Tables}

2.1. Thin disc scale length and scale height values from the literature. . . . . . . . . 13

2.2. Thick disc scale length/height values from literature . . . . . . . . . . . . 15

3.1. Table 1 from Robin et al. (2003). Defined initial mass function (IMF) and star formation rate (SFR) for all the model components . . . . . . . . . . . . 28

3.2. Table 4 from Robin et al. (2003). Age-velocity relations for the different components of the model. . . . . . . . . . . . . . . . . 30

3.3. Table 1 from Robin et al. (2003). Metallicity distributions assumed in original simulations: Age, mean metallicity, radial metallicity gradient and dispersion for each population. . . . . . . . . . . . . . . . . 32

3.4. SFR and IMF parameters in the standard and revised versions of the BGM. . . 33

3.5. Table 2 of Czekaj et al. (2014). Evolutionary tracks for the standard and revised versions of the BGM . . . . . . . . . . . . . . . . 34

3.6. Table 5 from Czekaj et al. (2014). Ingredients of the standard and revised sim-

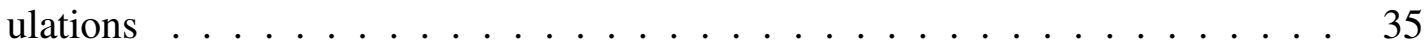

5.1. SEGUE survey plates used for the present analysis _ . . . . . . . . . . 48

5.2. Photometric flags used . . . . . . . . . . . . . . . . . . . . . . . . . . . . . . . . 48

5.3. Spectroscopic flags used . . . . . . . . . . . . . . . . . 49

6.2. Coefficients from the equation 6.2 for the correction to the DS extinction model. The different lines in each field are the different regions in the field where the program has been implemented. The regions were selected, from 1 to 9 , firstly from decreasing galactic latitude and then from increasing galactic longitude.

6.1. Mean, mode, and dispersion of the distribution of the total extinction in $\mathrm{V}$ band $\mathrm{A}_{V}$ before (B) and after $(\mathrm{A})$ correction for each field $\ldots \ldots 66$

6.3. Coefficients from the equation 6.2 for the correction to the Marshall extinction model. The different lines in each field are the different regions in the field were the program has been implemented. The regions were selected as in table 6.2 . 69

6.4. Regression laws for $\mathrm{S} / \mathrm{N}$ as a function of $\mathrm{r}$ magnitude. . . . . . . . . . . . . 72

6.5. Regression laws for radial velocity errors as function of $g$ magnitude for each plates. . . . . . . . . . . . . . . . . 73

7.1. Proper motions measures of central tendency (Mean and Mode) and of dispersion (Standard deviation) for the l-component . . . . . . . . . . . . 117 
7.2. Proper motions measures of central tendency (Mean and Mode) and of dispersion (Standard deviation) for the b-component . . . . . . . . . . . . . . . . 119

7.3. $\chi^{2}$ values (magnitude(m), color(c) and color-magnitude (c-m)) along with dispersion (for the standard model). Last three lines show the sum of $\chi^{2}$ values for all fields. . . . . . . . . . . . . . . . . . . . 122

7.4. Deegres of freedom (df) for magnitude(m), color(c) and color-magnitude(c-m).

8.1. The number of stars of the thick disc/thin disc in the standard model in each plate for the bright (b) and faint (f) plates after applying the selection sample. The number of stars in each plate and the mode of the total extinction $A_{V}$ for each distribution are also indicated. . . . . . . . . . . . . . . . . 126

8.2. Likelihood values for the spectroscopic parameters (MSTO stars) - original model128

8.3. Set of parameter range. The thick disc parameters have the subscript 'Thick', the old thin disc parameters have the subscript 'Old thin' and the thin disc parameters have the subscript 'Thin disc'.

8.4. Thick disc and thin disc metallicity mean values, when fitting the thick disc and old thin disc.The SN subscript refers to solar neighbourhood value. . . . . . . . 134

8.5. Thick disc metallicity mean values, when fitting the thick disc alone for case 2 . 137

8.6. Thick disc and thin disc metallicity mean set of parameters when fitting the thick disc along with all thin disc - for case $2 \ldots$. . . . . . . . . . 137

8.7. Likelihood values for the spectroscopic parameters with the new sets of parameters 139

8.8. Sum of the log likelihood values for all fields for the spectroscopic parameters (MSTO stars) with the new set of parameters.

8.9. Sum of the likelihood values, for groups of fields of different longitudes, for the spectroscopic parameters (MSTO stars) with the new set of parameters.

8.10. Thick disc and thin disc metallicity mean values, when fitting the thick disc (two slopes) and old thin disc. $R_{\text {change }}$ is the galactocentric radius at which the gradient of the thick disc changes.

8.11. Sum of the likelihood values, for different ages of the thick disc, for the spectroscopic parameters (MSTO stars) with the fitted parameters.

8.12. Thick disc and thin disc metallicity mean set of parameters - Fitting the old thin disc for the model $\mathrm{B}$ of the revised version.

8.13. Thin disc metallicity distribution from the literature . . . . . . . . . . . . . 146

8.14. Thick disc metallicity distribution from the literature . . . . . . . . . . . . 147

9.1. The value of the parameters used to compute the photometric errors in the simulation

9.2. Mean reddening for each of the observed disc fields in GES . . . . . . . . . . 150

9.3. Total extinction in each filter . . . . . . . . . . . . . . . 153

9.4. Number of stars, in the model, for the thin, young thick, old thick disc for each field after applying the selection sample. The total number of stars in each field is given in the last column 
9.5. Set of parameters used to compute absolute magnitude from the relation with effective temperature for dwarfs $(\operatorname{logg}>4.5 \mathrm{dex})$ and giants $(\operatorname{logg}<4.0 \mathrm{dex})$ and with $\log \mathrm{g}$ for subgiants ( $4.0 \mathrm{dex} \leq \operatorname{logg} \leq 4.5 \mathrm{dex})$. The selection in metallicity and $\log g$ is given in the first two columns respectively. . . . . . . . . . . . 162

9.6. GES metallicity distribution . . . . . . . . . . . . . . . . . 171

9.7. Set of parameter range. The thick disc parameters have the subscript 'Thick' and refer to the young thick disc. The subscript 'Old Thin' refers to the older ages of the thin disc as explained in section 8.1.5 . . . . . . . . . . . 175

9.8. Young thick disc and old thin disc metallicity mean values, when fitting the young thick disc and old thin disc with the GES survey . . . . . . . . . . . . 176 


\section{Chapter 1}

\section{Motivation}

The structure, formation and evolution of the Milky Way is still today under debate. Since Herschel and Kapteyn realized the importance of star counts the scientific community tried to create new and better observational surveys, but only in the last decades with the emergence of wide-field mosaic of CCD cameras it has been possible to create large complete catalogs. This large catalogs allowed the analysis and study of bright and faint magnitudes in vast areas of sky. This new large surveys create huge databases which allow a better exploration, star by star, of the Milky Way, as never done before and possibly not accessible for external galaxies in the near future. In this sense the Milky Way is a huge experimental laboratory from where we can obtain important information.

From the side of models and in particular stellar population synthesis models great effort has been done to make possible to understand data and test different scenarios of formation and evolution of the Milky Way. The existence of different models in the literature (Besançon Galaxy Model (Robin et al. 2003), Trilegal (Girardi et al. 2005), GALFAST (Jurić et al. 2008), Galaxia (Sharma et al. 2011)) is a proof of this work. The combination of the information taken from the comparison of data with different models is a further step to understand the Milky Way and realize our "place" in the Universe.

The goal of this study is to use a stellar population synthesis model the Besançon Galaxy Model (Robin et al. 2003) to understand data and selection bias, study the stucture of the Milky Way and test scenarios of formation and evolution of our Galaxy. In particular this work uses photometric data to study the shape and spectrocopic data to study the metallicity, surface gravity and effective temperature distributions in the different components of the Milky Way.

We organized the $\mathrm{PhD}$ manuscript in the following way: In the following chapter we begin to describe how important was astronomy in the ancient world and the efforts done, by different people, to have a better knowlodge of our place in the universe. We then depict the most accepted scenario of the formation of the universe and we briefly describe the different components of the Milky Way. In chapter 3 we introduce the BGM and describe the different ingredients used for each component. An overview of few surveys from the past, present and 
future is presented in chapter 4. Chapter 5 describes the photometric and spectroscopic sample used for this work. Chapter 6 explains how we proceed to obtain simulations, which we can compare with real observations, by masking, correcting extinction and applying errors. We then explain the selection applied to obtain Main Sequence Turnoff Stars and K giants from the SEGUE sample. Results for the photometric sample are given in chapter 7. We show qualitative and quantitative comparisons for magnitude, color, color-magnitude and proper motion distributions. We describe how we tried to constrain the IMF and the comparisons with the revised version of the Besançon Galaxy Model. Chapter 8 present the spectroscopic comparisons between simulations and observations, discuss the metallicity distribution and describe how we compute distances. This chapter also describes the ABC/MCMC method used to constrain the radial metallicity gradients and metallicity distributions from the thin and thick disc and the results. Chapter 9 describes the Gaia-ESO sample and $\mathrm{F} / \mathrm{G} / \mathrm{K}$ selection function, discuss the metallicity distributions and describes how we computed distances along with the results from the analysis. In the last chapter we discuss and present conclusions from our results and give some perspectives for future work. 


\section{Chapter 2}

\section{Introduction}

\subsection{Astronomy in the antiquity}

Astronomy dates to the antiquity, possibly to the pre-history and in the early times it was related to religion, mythology, calendars and general beliefs. The area of the science that studies these relations is called Archaeoastronomy, which is defined as the "anthropology of astronomy". Objects in the sky were first identified as gods or spirits in early cultures. The early development of science can be studied by the analysis of these surviving indigenous traditions and from the structures that early civilizations left. There are few ancient astronomical sites around the world (e.g., France (Carnac), Germany, China, Scotland, Turkey, Malte, Egypt) that have been discovered. The ones in Malte, called Hagar Qim and Mnajdra, are among the oldest ones (3600 and 2500 BC) following a period of development (Cox 2008). The temple in Egypts Sahara desert (6500 - 6000 BC) is known as Nabta and it consists of five megalithic alignments constructed by nomads ${ }^{1}$. The Gobeklitepe site in Turkey is probably the oldest known structure (c. 9600-7300 $\mathrm{BC})^{2}$. Why and by who it was constructed is still a mistery but its probable that it was a consequence of the development of that society and the need to understand the hereafter ${ }^{3}$. One of the most popular structures (around $2800 \mathrm{BC}$ ), probably a astronomical observatory with religious functions, is Stonehenge in which one can follow in the aligned rocks, motions of the Sun and Moon (Lang 2006). Meanwhile, in Mesopotamia, the first lunar calendar has been developed and culminated in a continuous record of solar and lunar eclipses (Lang 2006). In China, as in other regions of the earth, the sky was also source of wonder and mystery, which lead to an interest in observations. The Chinese, as the Babylonian, Egyptians, Hindus, Mayas, Celts among others, were among the first to record the sighting of a comet, the eclipse of the Moon, eclipse

\footnotetext{
${ }^{1}$ URL: http://www.colorado.edu/news/releases/1998/03/31/oldest-astronomicalmegalith-alignment-discovered-southern-egypt-science

${ }^{2}$ URL: http://www.gobeklitepe.info/

${ }^{3}$ David Lewis-Williams et David Pearce, « An Accidental revolution? Early Neolithic religion and economic change », Minerva, 174 (July/August, 2006), pp. 29-31
} 
of the Sun and create a calendar with 12 months of 30 days each ${ }^{4}$. First predictions were made in ancient Greece when Thales of Miletus (624 - 547 BC) estimated correctly a solar eclipse (around 585 BC) (Lang 2006). In this epoch the Greek civilization contributed for countless developments in astronomy as the planets constitution, shape, movement and placement. In 530 BC Pythagoras (572-479 BC) proposes a spherical shape for the earth, planets and Sun later modelized by Eudoxus of Cnidus (408-355 BC) which constructed a geocentric model of the solar system and stated that movements of stars in the sky where due to the Earth's rotation ${ }^{6}$. Later, Aristarchos of Samos (320-250) proposed that the Sun instead of the Earth is in the center of the solar system. The Heliocentric model was later celebrated by Nicolaus Copernicus (1473-1543) in the text De Revolutionibus and Johannes Kepler (1617-1621) text Astronomia nova. In 350 BC Aristotle (384-322 BC) proved that the Earth as a spherical shape as already proposed by Pythagoras. Among one of the important contributions, due to the Greeks, is the first known star catalog of 850 stars (129 BC), made by the Greek astronomer Hipparchus (190120 BC), later celebrated by Ptolemy (87-165). Meanwhile, astronomers began to understand that the universe is an hierarchical composition of different structures also called islands. In particular a milky patch of sky that rings the Earth, known in latin by Via Lactea which translates to road of milk, was of particular interest. This name was given due to the pale band of light formed by stars, gas and dust in the galactic plane as seen from Earth.

Eighteen-century astronomers were the first to realize that we make part of an enormous disk of stars, gas and dust which appears as a band around the sky due to the edge-on view from inside this disc. The Sun location in this structure was one of the first questions that arose. The first attempt to deduce the Sun location has been made by William Herschel (17381822). Around 1785 the first systematic survey of the sky was made by Herschel and his sister Caroline (1750-1848) in an attempt to deduce the structure of the Milky Way. The technique of star counts was being used for the first time and it was done for 683 regions of the sky. He reasoned that towards the galactic center the density of stars, compared with the average density, should be higher and towards the edge of the Galaxy should be lower. Figure 2.1 shows the map constructed by William and Caroline Herschel. They noticed that the lower density regions were outside the Milky Way which means that the shape is a disc. Because the density of stars was the same in all directions he concluded wrongly that the Sun is in the center of the Galaxy.

Later Jacobus Kapteyn (1851-1922) used observations fairly well represented, at least up to galactic lat. $70^{\circ}$ (Kapteyn 1922) that he called 'Selected Areas' to produce a catalog which he used to construct a higher precision map of the Milky Way than Herschel. Kapteyn introduced, in the catalog, motion, density, and luminosity to the Herschel's star counts map. Figure 2.2 shows the Kapteyn's map of the Milky Way.

The map shows that the Milky Way has a flattened disc with a radius of $8.0 \mathrm{kpc}$. The Sun is represented by a large circle around $2.0 \mathrm{kpc}$. Both, Herschel and Kapteyn, agree that the Milky Way is a flat structure with the Sun placed near the center but nevertheless the enormous

\footnotetext{
${ }^{4}$ URL: http://casswww.ucsd.edu/archive/public/tutorial/History.html

${ }^{5}$ URL: http://image.gsfc.nasa.gov/poetry/ask/a11846.html

${ }^{6}$ URL:http://casswww.ucsd.edu/archive/public/tutorial/History.html
} 


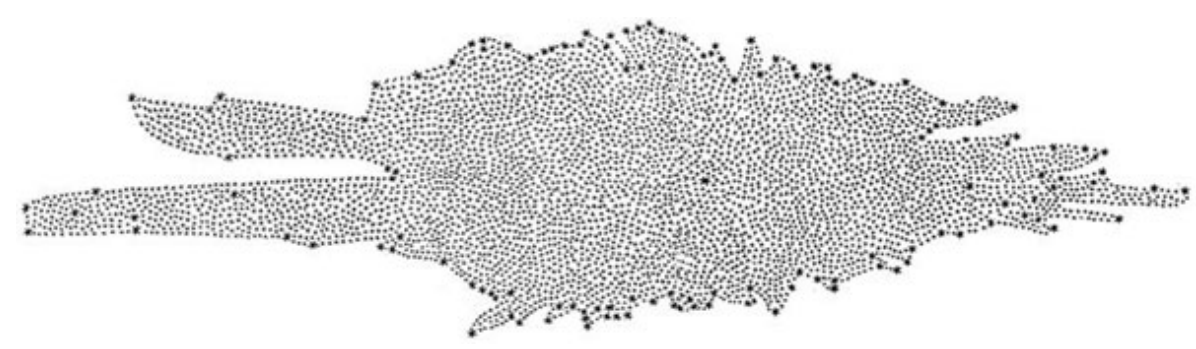

Figure 2.1: Herschel map of the Milky Way. The darker black point near the center of the map is the Sun position. Source: On the Construction of the Heavens. By William Herschel, Esq. F. R. S. Philosophical Transactions of the Royal Society of London, Vol. 75. (1785), pp. 213-266.

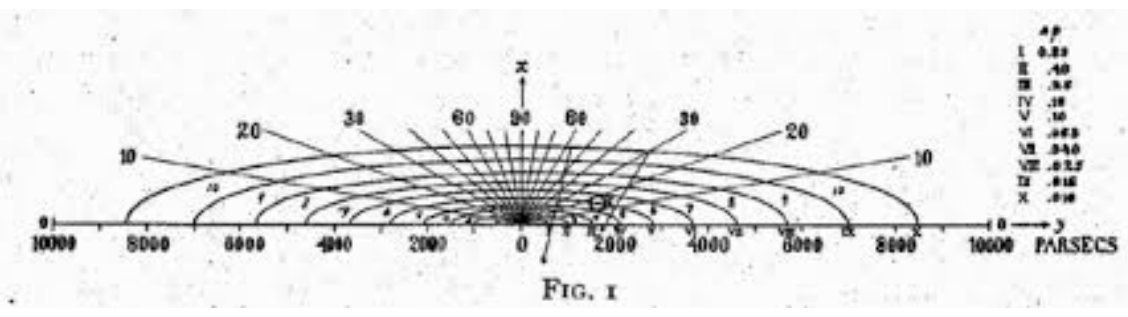

Figure 2.2: Kapteyn's map of the Galaxy. Source: Kapteyn (1922)

effort made by both the results were skewed because the authors were not aware of the strong extinction effect. In effect they were looking only to the nearest stars and had no idea about the actual size of the Galaxy. Later Harlow Shapley (1885-1972) analysing globular clusters RR Lyrae variables, computed distances and charted the distribution of globular clusters. $\mathrm{He}$ discovered that the Milky Way was at least two times larger than supposed by Kapteyn and that the globular clusters and stars in general orbited a common center far from the Sun in the direction of the Sagittarius constellation. In 1924, Edwin Hubble (1889-1953) at Mount Wilson made observations of Cepheids in the Andromeda Nebula and realized that it was too distant to be part of the Milky Way. It was demonstrated that the Milky Way is just one Galaxy among others in a large scale structure. This epoch defines the beginning of cosmology as a scientific area and of particular interest the formation of Galaxy's in a large scale structure as the Universe.

\subsection{Galaxy formation in a $\Lambda$ cold dark matter scenario}

The $\Lambda$ cold dark matter scenario (hereafter $\Lambda$ CDM) (e.g. Blumenthal et al. (1984), Springel et al. (2006), and references therein) is the most accepted scenario for the formation of the Universe. The haloes of CDM were formed from the density fluctuations after de Big Bang and its imprints remain visible in the Cosmic Microwave Background (hereafter CMB). Figure 2.3 show how the imprints from the early universe and specially the Baryon Acoustic Oscillations (BAO) are related with the large scale structure (Boughn \& Crittenden 2004; Springel et al. 2006). 


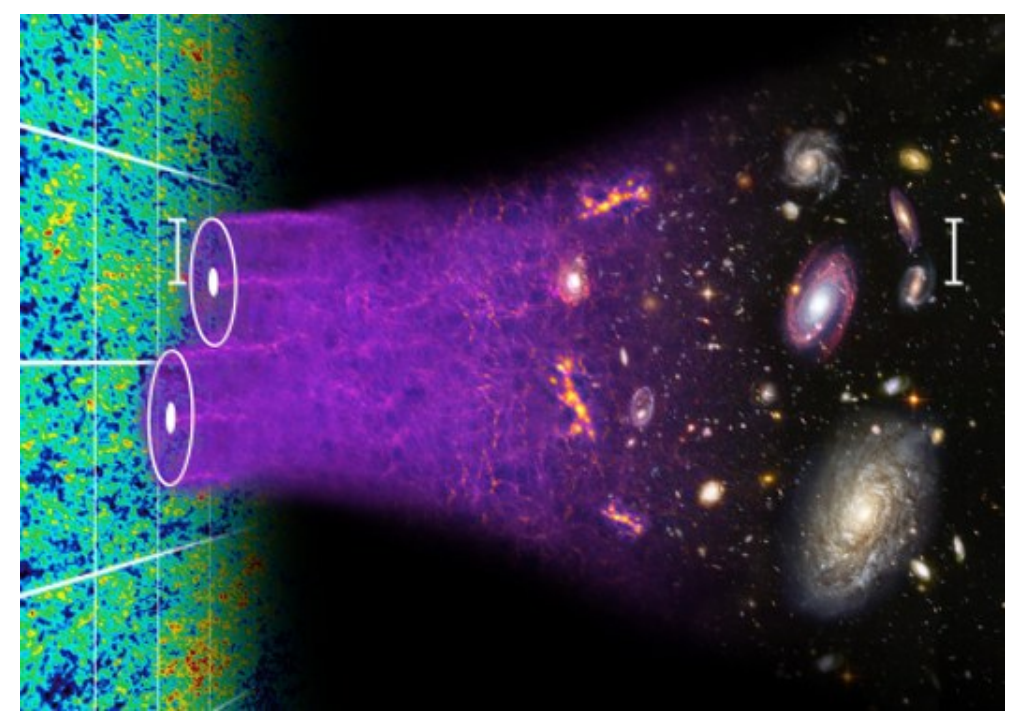

Figure 2.3: An illustration of the concept of baryon acoustic oscillations, which are imprinted in the early universe and can still be seen today in Galaxy surveys like the Baryon Oscillation

Spectroscopic Survey (BOSS; Schlegel et al. 2009). Image Credit: Chris Blake and Sam Moorfield in https://www.sdss3.org/surveys/boss.php

The visible structures of the universe are made of baryonic mass accreted in these haloes. At around $Z \sim 10$ star formation begins which drives gas out from the protogalactic dark matter mini-halos (e.g., Springel \& Hernquist 2003; Schultz et al. 2014). These first stars will belong to the stellar halo and are among the oldest stars in galaxies. As the time goes the hot gas, around the Galaxy, that is cooling and falling into the plane, gives rise to a rotating disc which at the beginning probably increases the Star Formation Rate. This scenario is well in agreement with large scale observations as the CMB provided by WMAP (Wilkinson Microwave Anisotropy Probe) (Spergel et al. 2007) but there are numerous predictions, at the scale of the Milky, which are not observed. We will briefly review few of them.

- Structure of the inner dark halo - A core or cusp shape?: The cuspy-halo problem arose in the early 1990s, when the first N-body simulations became available (Dubinski \& Carlberg 1991; Navarro et al. 1996, 1997). The simulations were better described by a steep power-law mass density distribution, like a cuspy distribution (Dubinski \& Carlberg 1991), instead of the observed core like behavior (see; de Blok 2010, for a general review).

- Number of predicted satellites (MSP): From predictions, a Galaxy as the Milky Way, should have around 500 satellites with masses higher than $10^{8} M_{\odot}$ (Moore et al. 1999). These objects were not still found in large numbers either in HI or visible studies. Lately there are some very faint satellite galaxies and dwarf spheroidal satellite galaxies which are dominated by dark matter being discovered (Mateo 1998; The Fermi-LAT Collaboration et al. 2013) but they are unlikely to arrive to the hundred. The large predicted number of satellites should leave a large number of debris of accreted satellites in the stellar disk and halo. An example of the active ongoing accretion history are stellar streams (Newberg et al. 2002; Belokurov et al. 2007) which were minor satellites disrupted by tidal forces. 
Several studies (Toth \& Ostriker 1992; Kormendy \& Fisher 2005; Kautsch et al. 2006; Stewart et al. 2008) showed that a large accretion activity, in particular the possibility of the interaction with a large merger, may be in disagreement with the presence of a strong disc as the Milky Way disc while Bournaud \& Elmegreen (2009) and Dekel et al. (2009) predict the stabilization (we mean in terms of its existence) of the disc after $z \sim 1$.

- Forming disks with small bulges in $\Lambda \mathrm{CDM}$ : In the $\Lambda \mathrm{CDM}$ scenario the formation of bulges is critical because its very difficult to generate galaxies without or with a very small bulge (van den Bosch 2001). Some recent advances have been made in this field. Brook et al. (2011) showed that in order to generate bulgeless disc galaxies the Galaxy has to expell large amounts of low angular momentum gas during merger events at early epochs which modifies the shape of the angular momentum distribution of baryons, from the original one given by the parent dark matter haloes, allowing the creation of smaller bulges.

- Angular momentum catastrophe (AMC): The lack of angular momentum of baryons (around 10\%), for SPH simulations of galaxies, in comparison with real galaxies and the predicted smaller disc sizes in comparison with real discs is called "angular momentum catastrophe" (Navarro \& Benz 1991; Navarro \& Steinmetz 1997; Sommer-Larsen et al. 1999; Navarro \& Steinmetz 2000).

- The Too Big to Fail problem: The Too Big to Fail problem (hereafter TBTF) is a particular case of the MSP. In simulations, where just Dark Matter is taken in account the central densities in subhalos are larger than the ones observed (Del Popolo et al. 2014).

Several individual solutions have been proposed for the listed problems. More recently Del Popolo et al. (2014), using the Del Popolo (2009) model, showed that the jointly small scale problems (cusp/core problem, MSP, TBTF problem, and the AMC) of the CDM scenario may be reconciled with observations by taking a semi-analytical model which takes in account, for the first time, the effect of ordered and random angular momentum, adiabatic contraction, dynamical friction, and the exchange of angular momentum between baryons and Dark Matter Del Popolo et al. (2014).

\subsection{Structure of the Milky Way}

The Milky Way is a barred spiral Galaxy composed of a disc (thin and thick disc), a bulge and a halo (stellar and dark matter halo) as showed in figure 2.4 (Buser 2000). The formation of the protogalaxy is still under debate but the most accepted scenario is one where the formation is hierarchical i.e. the halo is formed by satellite Galaxy accretion (Blumenthal et al. 1984; Springel et al. 2006), and references therein) (see section 2.2). The Milky Way has of the order of $1.0-2.0 \times 10^{11}$ stars and the dark matter halo can extend at least to $100 \mathrm{kpc}$ possibly being in contact with other galaxies dark matter haloes like Andromeda.Last measurements of the virial mass indicate values around $(1.26 \pm 0.24) \times 10^{12} M_{\odot}$ and total stellar mass around 


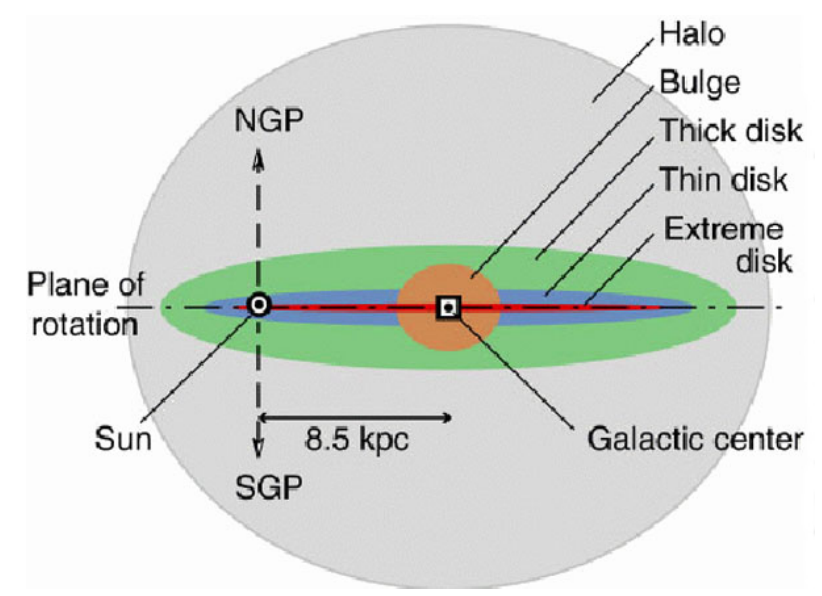

Figure 2.4: The Milky Way edge-on view showing the different components of the Milky Way with the Sun position indicated. Image Credit: R. Buser. Hurt

$(6.43 \pm 0.63) \times 10^{10} M_{\odot}($ McMillan 2011). The disc is not axisymmetric and the spiral arms nature, long-lived or transient, is still under debate. In recent past the disc was thought to be composed by two main arms (Perseus and Scutum-Centaurus arms) (Grabelsky et al. 1988), but the quantity of recent works indicating that the Milky Way have instead 4 arms increased to around $80 \%$ of the published papers on this question (Vallée 2014). The possible existence of 4 arms was first given from the analysis of HII regions (Georgelin \& Georgelin 1976) and more recently Vallee (2014) from a meta-analysis. The Sun is located in the range of $8.0 \mathrm{kpc}$ (Reid 1993; Brunthaler et al. 2011) to $8.5 \mathrm{kpc}$ (IAU value) from the galactic center and $15 \mathrm{pc}$ above the galactic plane (Hammersley et al. 1995; Freudenreich 1998; Drimmel \& Spergel 2001) as showed in figure 2.5. The artist also places the Galactic bar and a hypothetical second bar.

The edge of the disc is detected at a galactocentric distance of about $14 \mathrm{kpc}$ in some analysis (Robin et al. 1992; Ruphy et al. 1996; Minniti et al. 2011) but it is a scenario still under debate (Carraro 2014).

The nature and formation of the bulge of the Milky Way are still under debate. The bulge is elongated as a bar (Stanek et al. 1994; Dwek et al. 1995) and is inclined by an angle $\left(\sim 25^{\circ}\right)$ with respect to the line of sight. The side nearest to us is at positive galactic longitudes and the boxy bulges, as in our Galaxy, are associated with bars formed from bar-buckling instability of the disc (Saha \& Gerhard 2013).

Galaxys have also a large content of dark matter which was first proposed due to the Galaxy rotation problem. The discrepancy between theoretical predictions, assuming that the total mass is the sum of all the luminous components masses, and observations at large galactocentric radius lead to conclude the existence of a not luminous mass which was called dark matter (Rubin et al. 1978, 1980). Figure 2.6 shows the predicted and observed rotation curve for the M33 Galaxy. The discrepancy between the expected rotation from the visible matter and the observed one appears at larger radii.

The inclusion of a dark matter halo solves the question has seen in figure 2.7. 


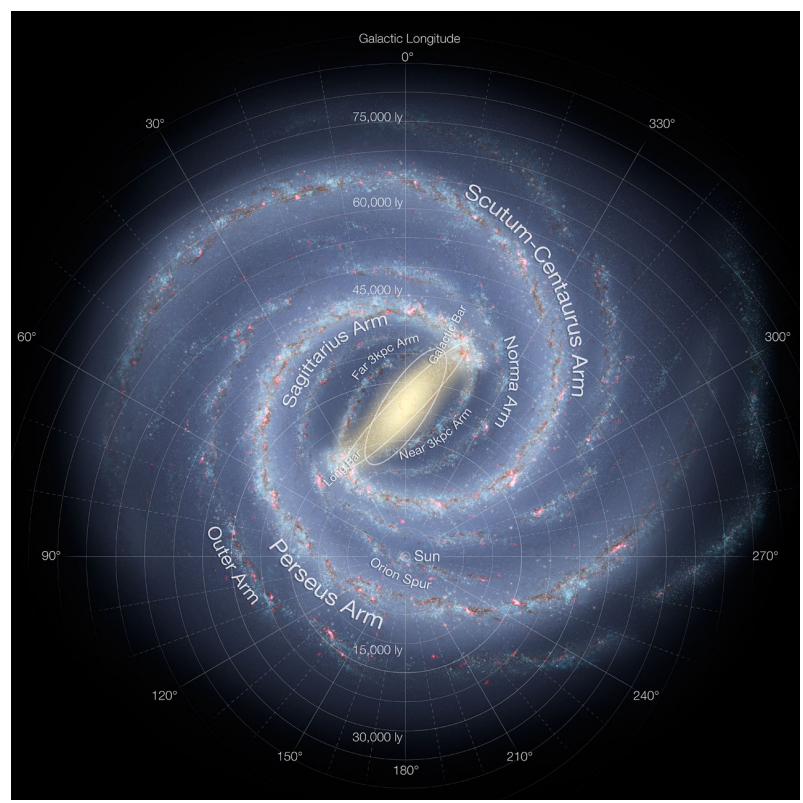

Figure 2.5: The Milky Way face-on view with the Sun position indicated. Artist's impression of the Milky Way. Image Credit: NASA/JPL-Caltech/ESO/R.

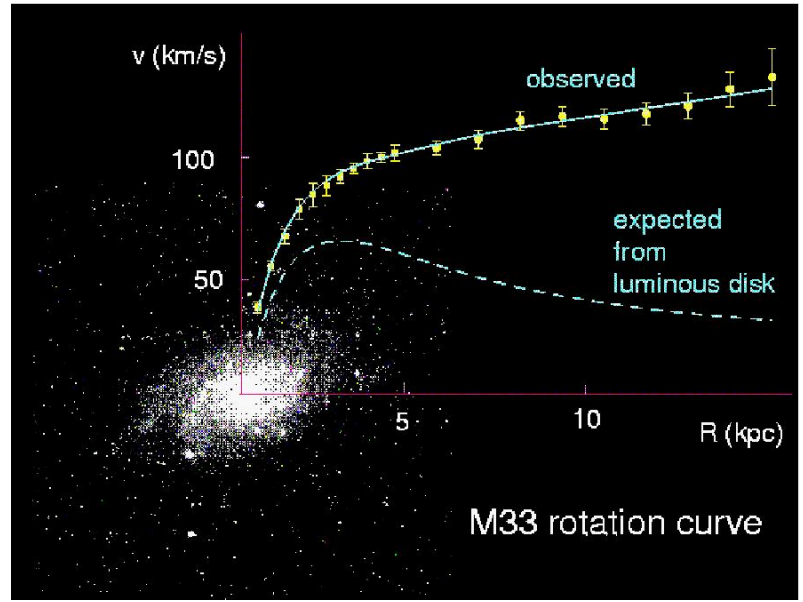

Figure 2.6: The observed and predicted rotation curve of the Galaxy M33. Image credit: University of Sheffield. 


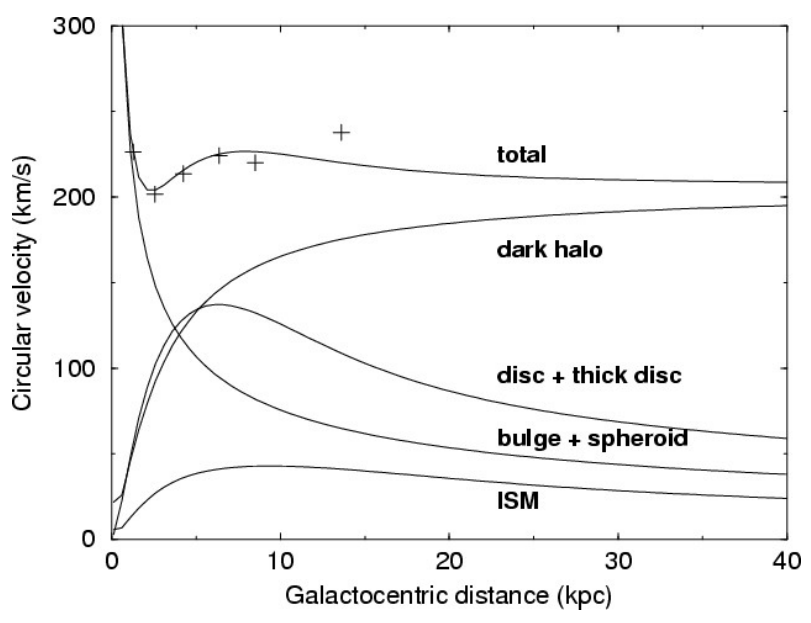

Figure 2.7: The predicted rotation curve from the self-consistent model of the Milky Way Robin et al. (2003). The contributions from each component of the Milky Way is also visible. The observations are from Caldwell \& Ostriker (1981)

\subsection{Galactic populations}

Working at Mount Wilson Observatory outside of Los Angeles and taking advantage of the Los Angeles blackouts during the second world war Walter Baade (1893-1960) resolved for the first time Andromeda Galaxy and both companions Messier 32 and NGC 205 (Baade 1944). Baade found that the stars fall into two different groups which he called population I and population II and concluded that population I stars, which are found in the solar neighbourhood, are mainly younger stars and can be found in sub-structures as open clusters while population II are found mainly in globular clusters. It is in 1957 in the Vatican conference that the definition of the two population was set. Stars from population I are younger more metal rich stars and seem to be constrained into the disc. Population II is composed of old metal poor stars that are homogeneously distributed in a sphere. The dynamics from both populations is also different as population I have circular orbits with small eccentricity and confined to the disc while population II stars have highly eccentric and inclined orbits (Figure 2.8). Later a population III was proposed for even older stars probably connected with the reionization epoch. The term stellar populations has evolved with time and nowadays, instead of classify stellar classes as decreasing metallicity and increase age (Ivezić et al. 2012), the term stellar population means a collection of stars which has a common spatial, kinematic, chemical, luminosity, and/or age distributions (Ivezić et al. 2012). 


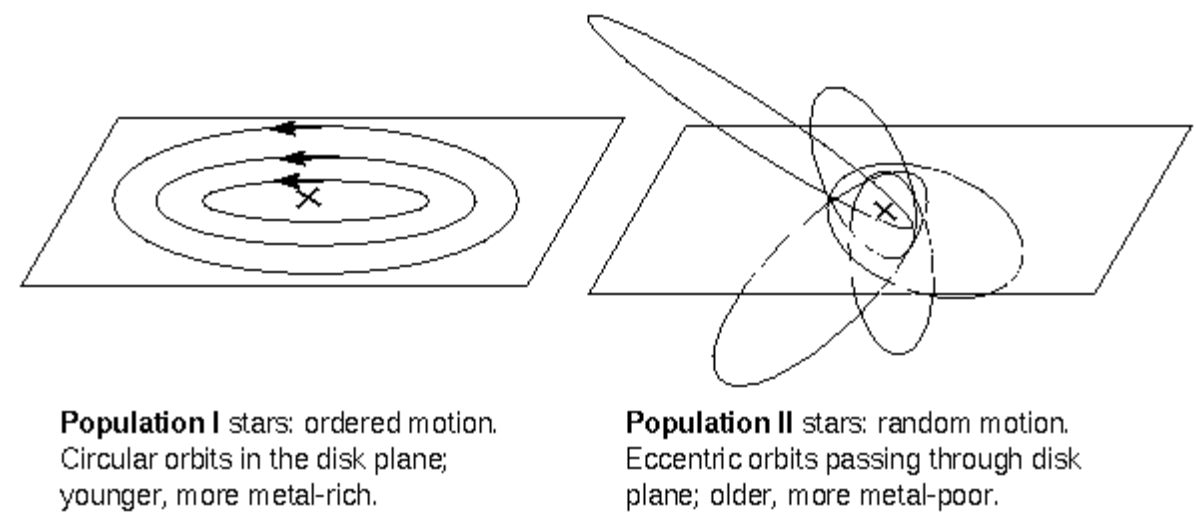

Figure 2.8: A scheme showing the orbits of population I and II.Image credit: Nick Strobel at www.astronomynotes.com.

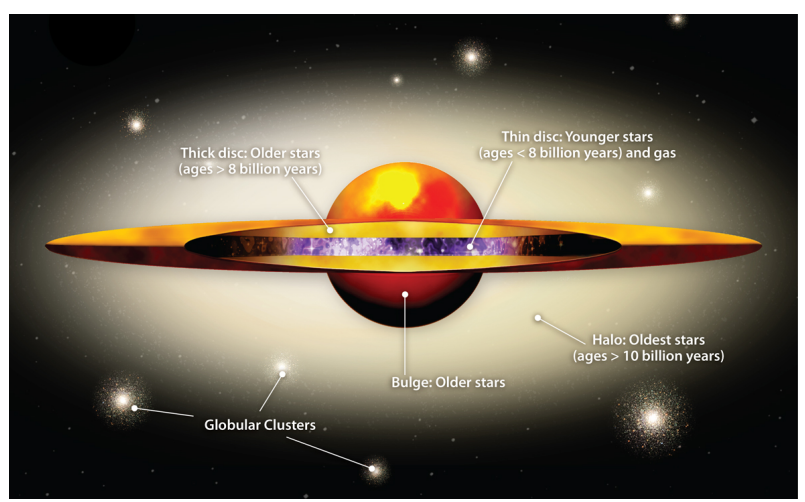

Figure 2.9: Schematic representation of a disc structure. Artist's impression of the Milky Way. Image Credit: Amanda Smith, IoA graphics officer

\subsection{Disc of the Milky Way}

The disc contains most of the dust, gas and stars of the Milky Way. The disc is flat, supported by rotation and shows a spiral structure delineated by gas and young stars. The stellar, gas and dust orbits lie in the plane of the Galaxy defined by the disc. Stars in the disc show a large range of ages and the mean peculiar motions of gas, dust and stars are small. The disc is the component of the Milky Way where the density of stars is higher. The stellar disc has a diameter of around $30 \mathrm{kpc}$ and the Sun is around $8 \mathrm{kpc}$ from the galactic center and $15 \mathrm{pc}$ above the galactic plane. The total mass of the disc is around $6.5 \times 10^{10} M_{\odot}$ (e.g., Flynn et al. 2006; Sofue et al. 2009; McMillan 2011). The local dark matter density is around $0.40 \pm 0.04 \mathrm{GeV} \mathrm{cm}^{-3}$ (McMillan 2011). The existence of two components in the disc is still under debate although it is generally accepted that there is a younger component formed essencially of population I stars called the thin disc and an older component, the thick disc, composed essencially of an intermediate population between population I and II stars. Figure 2.9 shows the decomposition of the disc into two different components as explained above. This decomposition is also seen in disc galaxies as showed in figure 2.10. 

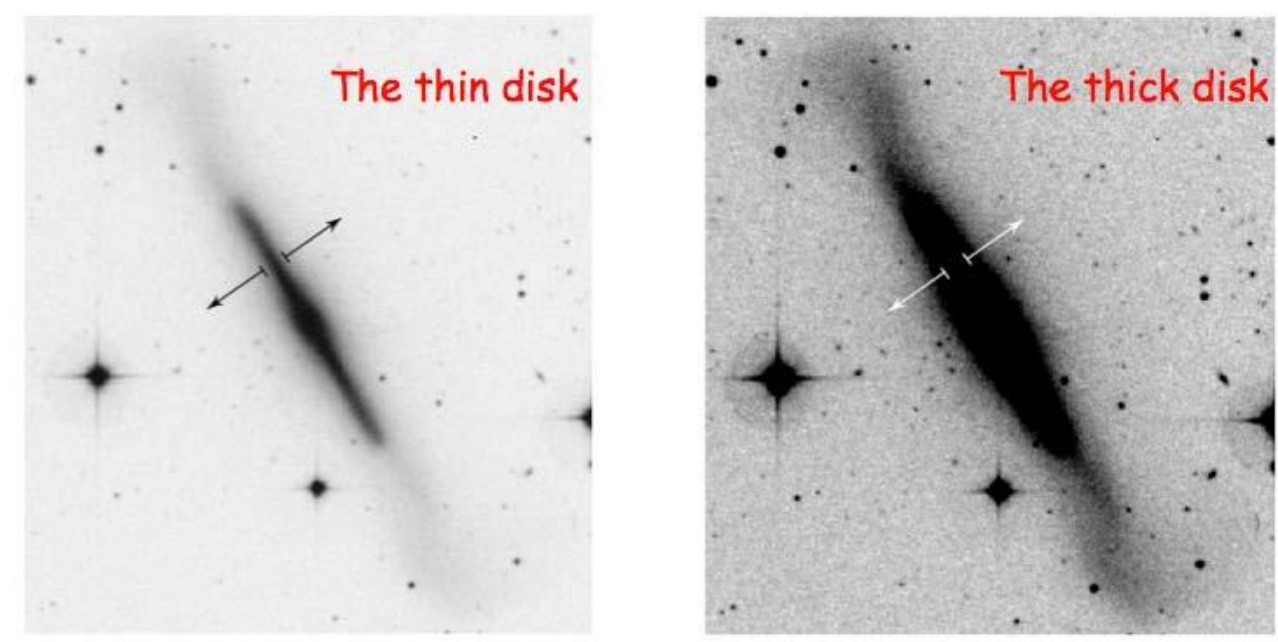

Figure 2.10: The NGC 4762 Galaxy, in different filters (B and V filters), showing a thin and a thick disc (Tsikoudi 1980). The image is taken from http://arxiv.org/ps/astro-ph/0208106v1 figure 3 in Freeman \& Bland-Hawthorn (2002)

\subsubsection{Thin disc}

The thin disc, contrary to the thick disc, has in its composition, apart of stars, large quantities of gas and dust. Stars in the thin disc have a wide range of ages from 0 Gyr to 10 Gyr either in open clusters or field stars (Bensby et al. 2003; Schuster et al. 2006; Haywood 2006a). The range of metallicities is $-1<[\mathrm{Fe} / \mathrm{H}]<0.4$ dex and the distribution peaks at the solar metallicity or slightly subsolar position ([Fe/H] -0.05 dex) (Haywood 2001, 2002; Taylor \& Croxall 2005; Luck \& Heiter 2006; Fuhrmann 2008; Ivezić et al. 2008; Casagrande et al. 2011). In contrast other analysis obtained a lower metallicity peak in the range -0.2 to -0.1 (Wyse \& Gilmore 1995; Rocha-Pinto \& Maciel 1996; Allende Prieto et al. 2004; Nordström et al. 2004; Holmberg et al. 2007). This component has currently the highest SFR of the Galaxy, specially in the arms of the Galaxy which triggered star formation in molecular clouds and open clusters. Therefore gas and young stars are very usefull tracers of the younger populations of the thin disc contrary to older stars that suffered perturbations and moved away from their original circular orbit. These young tracers are confined to 100 parsec above and below the plane and in particular OB spectral type stars can be found normally in open clusters or associations due to the larger mass needed to form these spectral types and smaller life-time (Carraro 2014). It is in the outer disc that we can find the older clusters $(\sim 5 \mathrm{Gyr})$ of the thin disc due to the larger stability and less interactions possible within that regions (Carraro et al. 2013, and references therein).

The thin disc scale length is still matter of debate with published values between 2 and 4 kpc. The scale height is around 250 pc (Kent et al. 1991; López-Corredoira et al. 2002; Jurić et al. 2008; McMillan 2011) which makes it a flattened structure. Table 2.1 resumes some values from the literature. 
Table 2.1:

Thin disc scale length and scale height values from the literature.

\begin{tabular}{|c|c|c|c|}
\hline Author & $\begin{array}{l}\text { scale length } \\
\mathrm{kpc}\end{array}$ & $\begin{array}{l}\text { scale height } \\
\text { pc }\end{array}$ & Comments \\
\hline Kent et al. (1991) & $3.0 \pm 0.5$ & $\begin{array}{l}\sim 247 \text { at } R_{\odot} \mathrm{kpc} \\
\sim 165 \text { at } R_{G C}=5\end{array}$ & $\begin{array}{l}\text { The Infrared Telescope (IRT) } \\
\text { (Koch et al. 1982; Melnick et al. 1987) }\end{array}$ \\
\hline (Ojha et al. 1994) & $2-2.5$ & & star-count samples at low-galactic latitude \\
\hline Chen et al. (1999) & $2.250 \pm 0.05$ & & 4 star-count samples at low-galactic latitude \\
\hline Ojha et al. (1999) & & $240 \pm 20$ & 14 star-count samples at low-galactic latitude \\
\hline Siegel et al. (2002) & $2-2.5$ & $\sim 280$ & 70000 stars in seven Kapteyn selected areas \\
\hline López-Corredoira et al. (2002) & $3.3 \pm 0.5$ & $285_{-12}^{+8}$ & low galactic latitudes from 2 MASS survey \\
\hline Bilir et al. (2006) & 1.9 & $\sim 221$ & intermediate galactic latitudes from the SDSS survey \\
\hline Jurić et al. (2008) & $2.6 \pm 0.52$ & $300 \pm 60$ & High galactic latitudes $\left(|b|>25^{\circ}\right)$ from the SDSS survey \\
\hline McMillan (2011) & $3.0 \pm 0.22$ & & MASER observations \\
\hline Bensby et al. (2011) & 3.8 & & 20 giants of the Magellan telescopes \\
\hline Chang et al. (2011) & $3.7 \pm 1.0$ & $360 \pm 10$ & High galactic latitudes $\left(|b|>30^{\circ}\right)$ from 2MASS survey \\
\hline Robin et al. (2012) & $2.2 \pm 0.165$ & & 2MASS data \\
\hline Bovy et al. (2012b) & $2.0-4.5$ & $200-1000$ & SEGUE G-dwarf sample \\
\hline Cheng et al. (2012a) & $3.4_{-0}^{+2.8}$ & & MSTO stars low latitudes from the SDSS survey \\
\hline Bovy \& Rix (2013) & $2.15 \pm 0.14$ & & SEGUE G-dwarf sample \\
\hline Polido et al. (2013) & $2.120 \pm 0.2$ & $205 \pm 40$ & All sky from the 2MASS survey \\
\hline Lopez-Corredoira \& Molgo (2014) & $2.0_{-0.3}^{+0.3}$ & $240_{-0.01}^{+0.12}$ & $|l|>50^{\circ}|b|<23^{\circ}$ from the SEGUE SDSS survey \\
\hline
\end{tabular}

Stars in the thin disc have high rotational velocities, low vertical velocities and quasi-circular orbits. The circular velocity of the group of stars, moving with the Sun, at the solar position is defined to be the Local Standard of Rest velocity $\left(V_{L S R}\right)$. This is computed by taking the mean of the group of stars and is about $220 \mathrm{kms}^{-1}$ (e.g. Kerr \& Lynden-Bell 1986; Dehnen \& Binney 1998) with small dispersion velocities of around $25 \mathrm{kms}^{-1}$ (Gomez et al. 1997). The asymmetric drift is the tendency, for a group of stars, with a mean rotation velocity around the galactic center, to lag behind stars from the local standard of rest. The asymmetric drift equations have been derived by Mihalas \& Binney (1981); Binney \& Tremaine (2008a). See Rix \& Bovy (2013) for a general review about the thin disc.

\subsubsection{Thick disc}

The question about the existence of an intermediate population (between pop II (the halo) and pop I (thin disc)) arose in the 40's with Baade (1944) and further Baade (1958), using disc globular clusters. Later, Gilmore \& Reid (1983) and Robin \& Crézé (1986) showed evidences for the existence of this intermediate population in contrast with Bahcall \& Soneira (1984) who argued that observations could be explained without the need of a thick disc population. Since this epoch a number of evidences in favor of the thick disc have been found. Several works noticed that the galactic disc is the sum of two components with different chemical compositions, structure and kinematics (e.g., Gilmore et al. (1995), Chiba \& Beers (2000), Bensby et al. (2003, 2004a,b,c), Ivezić et al. (2008)). 


\section{Shape}

The shape of the thick disc depends on the density decrease as a function of the distance from the galactic center and perpendicular distance to the galactic plane. The shape of the thick disc may be modelled by an exponential or a $\operatorname{sech}^{2}$ law. The value of the scale length is still under debate with the existence of a large range of values in the literature but the latest results point to a scale length comparable with the old thin disc one, about $2 \mathrm{kpc}$, while the younger thin disc has a larger scale length as visible in table 2.2. The scale height is less than half the scale length and the dispersion in the cited values is also large with values ranging between $500 \mathrm{pc}$ to $1500 \mathrm{pc}$ (Table 2.2). It has been claimed in the past that the thick disc is a separate population from the thin disc with a different formation scenario. In a more recent paper Bovy et al. (2012a) claimed that there is a continuity between thin and thick discs. They found a continuous distribution of scale heights for the disc which indicates a smooth transition between the two populations and disfavour a bi-modal composition of the disc. The source of the disagreement between works can be due to the difficult separation between thin and thick disc on one side and thick disc and halo on the other side.

\section{Age}

In the literature the formation of the thick disc has values in the range of 8 Gyr (Ibukiyama \& Arimoto 2002) to 13 Gyr (Pettinger et al. 2001). The duration of the thick disc formation may have been short (Mashonkina \& Gehren 2001) (not longer than 1 Gyr) but several recent results (Bovy et al. 2012b; Haywood et al. 2013, among others) claim a longer thick disc formation period (4-5 Gyr). Therefore the thick disc has an age that is intermediate between of the older halo and the younger thin disc.

\section{Kinematics}

Stars of the thick disc have more eccentric inclined orbits and lower rotational velocities as compared to the thin disc. Ojha et al. $(1996,1999)$ derived velocities for the thick disc component from a UBV photo-astrometric survey in three different directions (center, anticenter and rotation) at intermediate latitudes. The derived velocity dispersions were higher compared with the ones from the thin disc. The high rotational lag of about $53 \mathrm{kms}^{-1}$, found in this work as in more recent analysis (Soubiran et al. 2003) is a proof of the kinematical difference between thick and thin disc components. In the past the thick disc was tentatively distinguished by its kinematics (e.g. Bensby et al. (2003), Soubiran et al. (2003)), but it appears most recently, thanks to high resolution spectroscopy, that the thick disc can be better distinguished from the thin disc by its $\alpha$ abundance (e.g. Bensby et al. (2011), Adibekyan et al. (2013)). 
Table 2.2:

Thick disc scale length/height values from literature

\begin{tabular}{lcc}
\hline \hline Author & $\begin{array}{c}H_{R} \\
(\mathrm{kpc})\end{array}$ & $\begin{array}{c}H_{z} \\
(\mathrm{pc})\end{array}$ \\
\hline Gilmore \& Reid (1983) & & 1350 \\
Reid (1993) & & 1500 \\
Soubiran (1994) & & 700 \\
Robin et al. (1996) & 2.8 & 760 \\
Spagna et al. (1996) & 3.0 & 1137 \\
Buser et al. (1999) & 4.5 & \\
Chiba \& Beers (2000) & 3.7 & 860 \\
Ojha (2001) & 2.5 & 900 \\
Vallenari et al. (2006) & & $600-1000$ \\
Du et al. (2006) & & 1500 \\
Girard et al. (2006) & & 1200 \\
Robin et al. (2007) & 3.6 & 900 \\
Jurić et al. (2008) & & 1048 \\
Veltz et al. (2008) & 2.2 & 510 \\
Carollo et al. (2010) & 4.1 & 750 \\
de Jong et al. (2010) & 3.4 & 694 \\
Kordopatis et al. (2011) & 1.96 & 655 \\
Bovy et al. (2012b) & 1.8 & \\
Cheng et al. (2012a) & 2.5 & 710 \\
Lopez-Corredoira \& Molgo (2014) & 2.3 & 535 \\
Robin et al. (2014) (12 Gyr) & 3.0 & 800 \\
Robin et al. (2014) (Old thick disc - 12Gyr) & \\
Robin et al. (2014) (Young thick disc - 10Gyr) & 2.0 & 350 \\
\hline \hline
\end{tabular}




\section{Metallicity and Chemical abundances}

The metallicity of the thick disc has been studied is different works and there is indication of a mean metallicity value around $-0.5 \mathrm{dex}$ and the non existence of a radial metallicity gradient. The metallicity dispersion is also large, around 0.30 dex and there are stars than may have a very low metallicities around -1.5 dex. Table 8.14 shows mean metallicity and radial gradients in the thick disc from the literature. The chemical abundances are also different from the ones of the thin disc. $\alpha$ elements are elements with $\mathrm{Z} \leq 22(\mathrm{C}, \mathrm{N}, \mathrm{O}, \mathrm{Ne}, \mathrm{Mg}, \mathrm{Si}, \mathrm{S}, \mathrm{Ar}$, $\mathrm{Ca}, \mathrm{Ti}$ ) which are synthesized by triple-alpha process (produce carbon and some oxygen) or alpha capture. For a determined metallicity stars that belong to the thick disc will have a larger alpha over iron value compared to thin disc stars (e.g. Bensby et al. 2011; Adibekyan et al. 2013) which suggest a older and fast formation of the thick disc (not much longer than $1 \mathrm{Gyr}$ from the $[\mathrm{Eu} / \mathrm{Mg}]$ values (Mashonkina \& Gehren 2001)). The thick disc, compared with the thin disc, follows a distinct sequence in most $\alpha$ elements (Oxygen, magnesium, calcium among others). The different chemical abundances for these two components are linked with different star formation histories because the star chemical composition is equal to the interstellar matter chemical composition from which stars are born. In this sense the chemical composition in old low-mass stars is a good tracer of the early interstellar medium chemical composition. Moreover, the chemical composition of the interstellar medium changes with time due to the release to the interstellar medium of different chemical elements produced in stars with different masses. The iron is mainly and in large quantities produced in supernovae type Ia, which in general is the explosion in a binary system composed by a white dwarf and a giant (can be other type of star) (For a review: Nomoto et al. 1994). The white dwarf may accrete material from the companion until the Chandrasekhar limit is reached giving rise to an explosion which releases iron in the interstellar medium. This type of supernovae expels iron in the interstellar medium at longer time scales (Nomoto et al. 1997) than the supernovae type II which inject $\alpha$ elements at shorter time scales because this elements are produced in very massive stars with short life-times (Arnett 1996) changing the chemical composition of the interstellar medium with time. There are also the asymptotic giant branch stars (AGB) which produce and inject elements in another time scale (Simmerer et al. 2004). Therefore, in the early times, the interstellar medium is enriched with $\alpha$ elements and less with iron which results on a large $\alpha$ over iron ratio and a low metallicity for the born stars. As the age of the Milky Way increases the quantity of expelled iron increases and the ratio between $\alpha$ elements and iron becomes smaller and the metallicity increases. Therefore, older stars have lower metallicities and higher $[\alpha / \mathrm{Fe}]$ ratios and younger stars with higher metallicities and lower $[\alpha / F e]$ ratios. Therefore, the $[\alpha / \mathrm{Fe}]$ vs $[\mathrm{Fe} / \mathrm{H}]$ sequence gives informations about the $\mathrm{SFH}$, and give constraints on to differentiation of the thin and thick discs which means that the sample selection by elemental abundance e.g. $[\alpha / \mathrm{Fe}]$ and $[\mathrm{Fe} / \mathrm{H}]$ select stars independently from there kinematical or positional properties. This new criteria can help us to understand better the formation and evolution processes of the Milky Way. 


\section{Formation Scenarios}

If the thick disc is identified to be a distinct population from the thin disc, it implies different formation scenarios for both components. There are several scenarios to explain the formation and evolution of the thick disc, here we refer to the five most accepted. Two of these scenarios require the presence of extragalactic mergers. (1) The first of these scenarios is direct accretion of stars from satellites (e.g., Statler 1988; Toth \& Ostriker 1992; Quinn et al. 1993; Velazquez \& White 1999; Abadi et al. 2003). (2) The second is the vertical heating, of a pre-existing thin disc, by minor mergers (e.g., Kazantzidis et al. 2008; Read et al. 2008; Villalobos \& Helmi 2008; Purcell et al. 2009; Bird et al. 2012). Cosmological simulations show that the mergers necessary to support these scenarios are very common. Observational evidences for the possibility of mergers in the Milky Way are given by the detection of streams in the halo (e.g., Newberg et al. 2002; Belokurov et al. 2007). (3) One scenario that does not require the presence of extragalactic mergers is in situ formation of the thick disc from homogeneous gas. High gas accretion rates can create an early turbulent disc phase. As a result a well mixed primordial gas disc can rapidly form stars at an efficient rate at early times forming a chemically homogeneous thick disc (e.g., Brook et al. 2004; Bournaud et al. 2009). (4) A scenario that has been extensively proposed in the literature is the thin disc thickening by bar and/or spiral instabilities. Spitzer \& Schwarzschild (1951, 1953); Eggen et al. (1962); Dennis (1966); Wielen (1977) observed the age-velocity dispersion correlation in solar neighbourhood stars originated by the scattering of stars from their initial circular orbits into more eccentric and inclined orbits. To explain the vertical heating of the thin disc some mechanisms were proposed like molecular clouds (e.g., Mihalas \& Binney 1981; Jenkins \& Binney 1990; Jenkins 1992), along with a transient spiral structure (e.g., Barbanis \& Woltjer 1967; Sellwood \& Carlberg 1984) or along with halo black holes (e.g., Hänninen \& Flynn 2002, 2004; Carlberg \& Sellwood 1985), black holes (Lacey \& Ostriker 1985), dark clusters (Carr \& Lacey 1987), infall of satellite galaxies (Velazquez \& White 1999), through 'popping' star clusters (Kroupa 2002), interaction of multiple spiral density waves (Minchev \& Quillen 2006) and spirals along with a bar (Minchev \& Famaey 2010). (5) Sellwood \& Binney (2002) suggest that radial migration can redistribute stars in the disc. In this case the thick disc formation is the result of a secular process related to the spiral arms and the bar (Schönrich \& Binney 2009a,b) (hereafter SB09a,b). Some observational works invoked radial migration in order to explain stellar properties at the solar neighbourhood (e.g., Haywood 2008; Loebman et al. 2011).

Chemical and kinematic properties are true witnesses of galactic evolution. Chemodynamical models (e.g., Schönrich \& Binney 2009a,b; Minchev et al. 2011, 2012, 2013) are efficient tools to understand radial migration. However various approaches give contradictory results. For example in Minchev series of articles, in contradiction with SB09a,b, it is demonstrated that radial migration is inefficient to promote disc thickening. It contributes only for the formation of a flared disc. According to the latter authors there are two oversimplifications made in SB09a,b which affected their results and conclusions: The not inclusion of a central bar (1) and the conservation of the vertical energy for migrating objects (2). The former oversimplification results in a lack of momentum changes at short scale-lengths. Nevertheless, in their model, the radial migration efficiency extends to short galactocentric distances $(\mathrm{R}<2 \mathrm{kpc})$ which mimics the effect of a bar. The latter affects one of their main conclusions, the thick disc can emerge in 


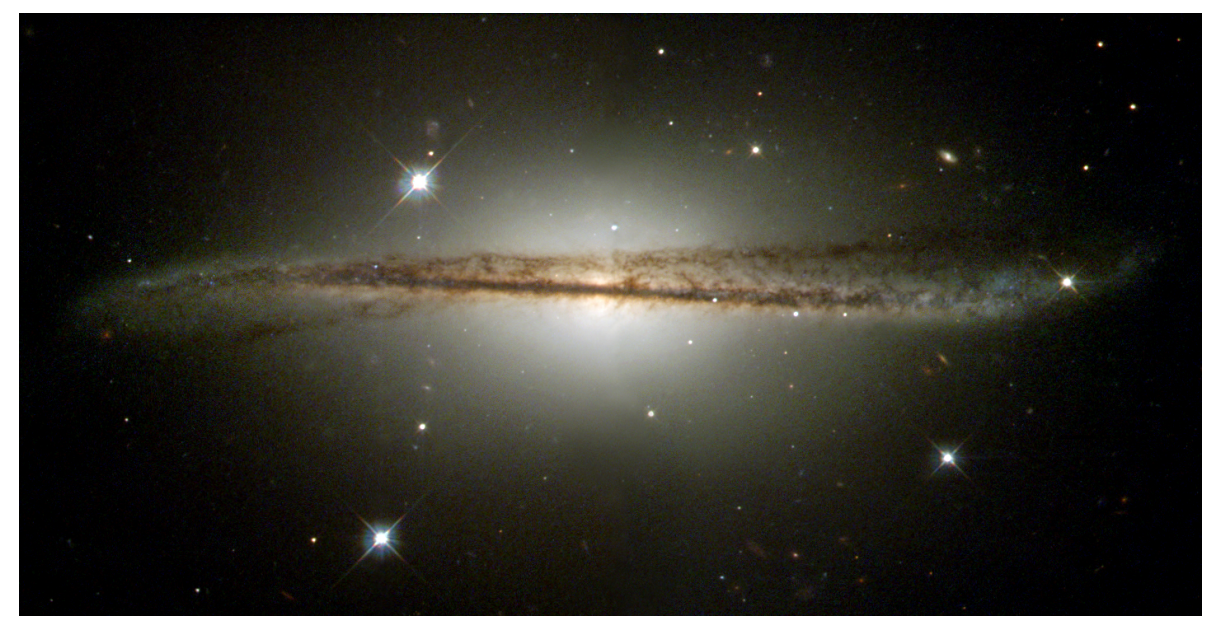

Figure 2.11: Warp of the spiral Galaxy ESO 510-13. Image Credit: NASA's Hubble Space Telescope

an isolated disc.

\section{Warp of the Milky Way}

The warp of the disc is a distortion in the external disc of the Milky Way, present in many external galaxies (Bosma 1991), as showed in figure 2.11. The warp has been studied by diverse authors and evidences point for a warp, as a structure, existent in all Milky Way components, dust, gas and stars.

The gas (atomic hydrogen) warp was first reported in works from Henderson et al. (1982), Burton \& te Lintel Hekkert (1986) and Diplas \& Savage (1991) and more recently discussed in Nakanishi \& Sofue (2003) and Levine et al. (2006) using radio observations. The method consisted in observing the $21 \mathrm{~cm}$ line of atomic hydrogen in order to project in the galactic plane the surface density of $\mathrm{HI}$ gas and compute the HI distribution as a function of the distance above the plane. Results showed the lack of symmetry in the surface density which is a consequence of a warping disc. Nakanishi \& Sofue (2003) found that the HI warp is asymmetric, because it goes higher above the galactic plane in the northern hemisphere. Levine et al. (2006) described the gas warp using a superposition of Fourier modes. For galactocentric distances smaller than $15 \mathrm{kpc}$ the first mode has more power. The mode equals to zero and two rule for galactocentric distances superiors to $15 \mathrm{kpc}$ which results in an asymmetric warp.

The dust warp was first detected by Freudenreich et al. (1994) using the Diffuse Background Experiment (DIRBE) of the cosmic Background Explorer (COBE) (Hauser et al. (1991), Boggess et al. (1992), Silverberg et al. (1993)). Later, Drimmel \& Spergel (2001) used the same experiment to model the dust distribution and concluded the presence of a warp in dust and stellar components which starts at the solar circle. In Drimmel \& Spergel (2001) the author assumes that the warp is well described by a quadratic function. Marshall et al. (2006) used The Two Micron All Sky Survey (2MASS: Skrutskie et al. (2006)) along with the Besançon Galaxy Model 

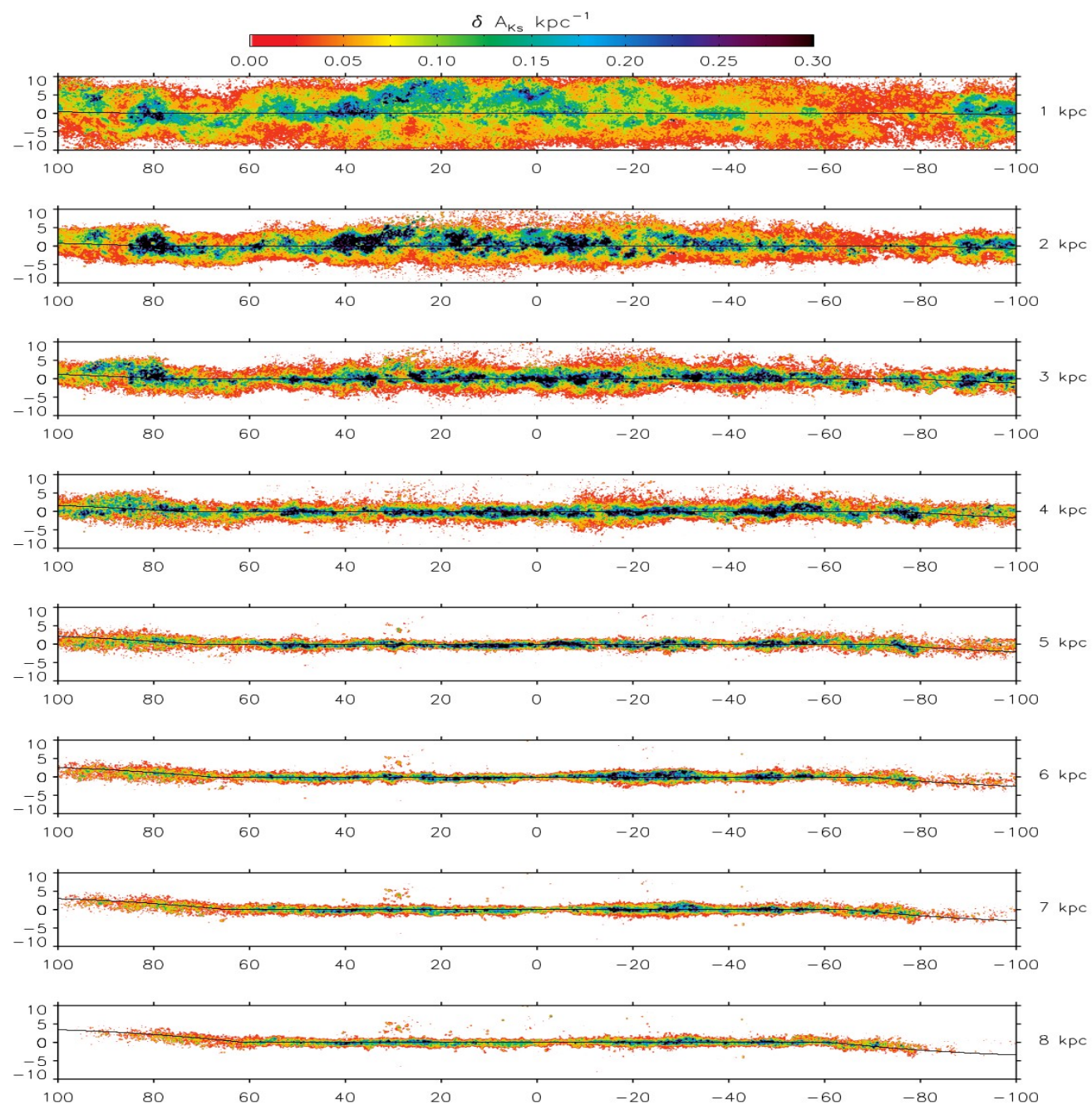

Figure 2.12: The dust warp, in galactic coordinates, as traced by extinction from figure 8 of Marshall et al. (2006). Each panel describes the extinction at $1 \mathrm{kpc}$ intervals from the nearest (top panel) to the further (bottom panel) distance. The black line indicates the position of the mid plane as given by the stellar warp formula in Robin et al. (2003).

to develop a 3D extinction map to know what was the density distribution of absorbing material across the plane of the Milky Way (Fig 1 Marshall et al. (2006)). They concluded that, as the CO and HI observations of the ISM (Nakanishi \& Sofue 2003), the dust distribution is found asymmetrically warped. In figure 2.12 Marshall et al. (2006) showed the dust warp as traced by extinction.

The warp in stars was first investigated in Djorgovski \& Sosin (1989) using the Infrared Astronomical Satellite (IRAS: Neugebauer et al. (1984)). Gyuk et al. (1999) used the Wide Field Camera from Hubble Space telescope (HST: Santiago et al. (1996), Mendez \& Guzman (1998), Gould et al. (1998)) and found that in order to make models consistent with observations the warp has to be very asymmetric. Later Alard (2000) used 2MASS and concluded that the stellar disc warp has a similar behavior compared to the gas warp. From the analysis of star 
counts in the DENIS survey (Epchtein et al. 1999), Derriere \& Robin (2001) noticed also the existence of a warp and a flare in the stellar disc. López-Corredoira et al. (2002) used the 2MASS survey and noticed the existence, in the old stellar population, of a warp which has an amplitude similar to the gas warp. Reyle et al. (2009) using the 2MASS survey along with the Besançon Galaxy model (Robin et al. 2003) found that at positive longitudes the galactic warp is well reproduced by a S-shaped model but at negative longitudes no simple model can reproduce well the observations. This was interpreted as a feature of the asymmetric warp. The amplitude of the asymmetry in the warp seems to be dependent of the component one is analysing. The dust warp seems to be less pronounced (e.g. Drimmel \& Spergel (2001), Marshall et al. (2006)) than the stellar warp (e.g. Drimmel \& Spergel (2001), López-Corredoira et al. (2002)) which is less pronounced than the gas warp (e.g. Nakanishi \& Sofue (2003), Levine et al. (2006)).

The origin of the warp is still unknown but there are different scenarios that try to give an explanation. (1) Tidal forces originated from nearby galaxies, like the Magellanic clouds or the Sagittarius dwarf (Weinberg \& Blitz 2006), (2) infalling intergalactic gas (López-Corredoira et al. 2002)) or (3) a misalignement of the angular momenta between the dark halo and the disc (e.g. Bailin (2003) Bailin \& Steinmetz (2003)). Gaia will measure the stellar kinematics in the region of the warp, allowing to constrain the dynamics of the stellar disc warp (Perryman et al. 2014).

\section{Modeling the stellar warp}

Different authors tried to model the warp using different shapes and parameters. In the S-shaped warp tilted ring model from Porcel et al. (1997), the distance of the true mid-plane to the plane defined by latitude $b=0^{\circ}$ is computed using eq. 2.1, where $h_{w a r p}$ is a linear function of the galactocentric radius. This is the model implemented in the Besançon Galaxy Model and it will be better explained in section 3.2.2. The stellar warp described in Drimmel \& Spergel (2001) assumes a quadratic function of the galactocentric distance. They defined $Z_{\text {warp }}$ as the vertical displacement of the warp.

$$
Z_{\text {warp }}=h_{\text {warp }}(r) \sin \left(\phi-\phi_{\text {warp }}\right)
$$

where $\phi_{\text {warp }}$ is the phase of the warp and the amplitude $h_{\text {warp }}(r)$ is described by

$$
h_{\text {warp }}(r)=\left\{\begin{array}{l}
0 \text { if } r \leqslant r_{\text {warp }} \\
a_{\text {warp }} \times\left(r-r_{\text {warp }}\right)^{2} \text { if } r>r_{\text {warp }}
\end{array}\right.
$$

$r_{\text {warp }}$ is the galactocentric distance at which the warp starts and $a_{\text {warp }}$ is an amplitude parameter equal to $27.4 p c k p c^{-2}$ (Table 2: Drimmel \& Spergel 2001) for the stellar warp. At a galactocentric distance equal to $12 \mathrm{kpc}$, the maximum height above the plane is equal to $685 \mathrm{pc}$. López-Corredoira et al. (2002) assumed a different shape of the warp, fitted of 2MASS data. At $10 \mathrm{kpc}$ Lopez-Corredoira's warp goes up to 214 pc while DS warp goes to $110 \mathrm{pc}$. 


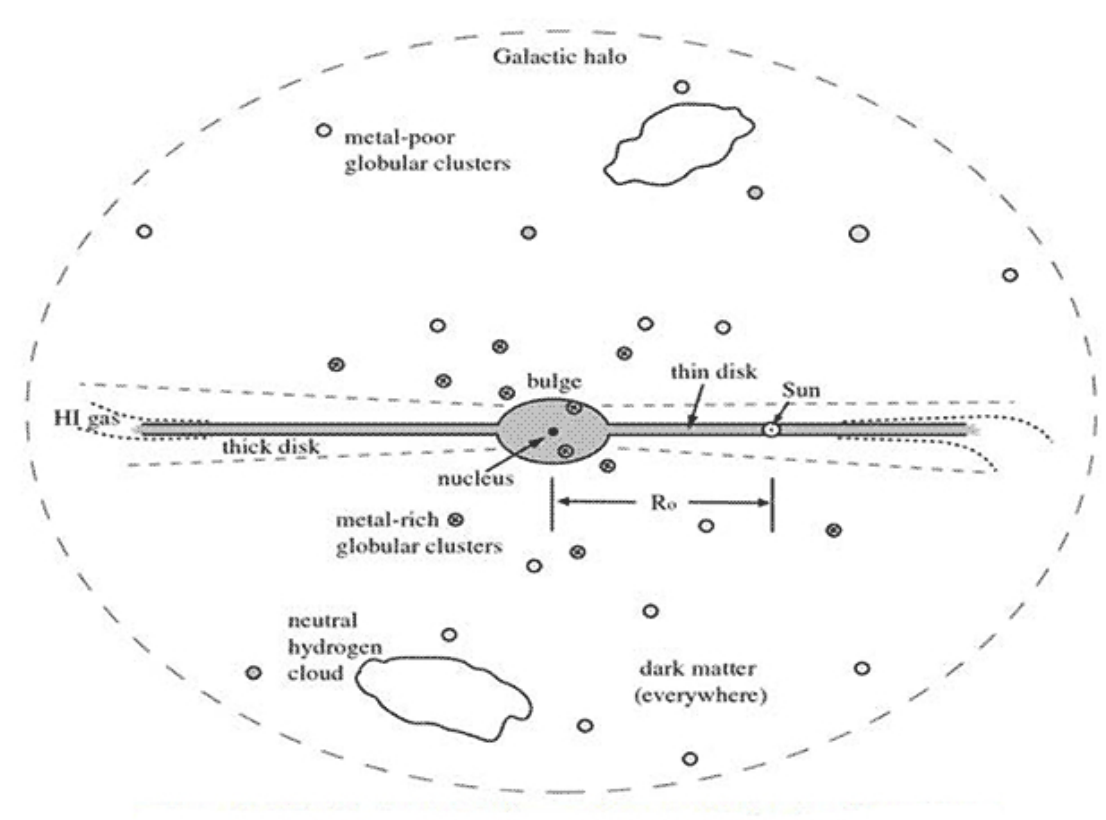

Figure 2.13: Schematic representation of the galactic structure. The flare is represented by the discontinuous line representing the thick disc which increases scale height as a function of the galactocentric radius. Image Credit: Gaia-ESO Survey

\subsection{Flare}

The flare is the increase of the scale height of the disc as a function of the galactocentric radius (Gyuk et al. 1999) as showed in figure 2.13. In this schematic representation we can see also the representation of the warp distortion at large distances from the galactic center.

The possibility of the existence of a flare in the gas was first reported in Henderson et al. (1982) and followed by Burton \& te Lintel Hekkert (1986) where the authors could not explain easily the origin of microlensing events toward the Large Magellanic Cloud (LMC). Later, the flare was studied by Gyuk et al. (1999) using star counts from the HST and Derriere \& Robin (2001) with DENIS survey. The later stated that the minimum radius for the flare depends on galactic longitude but in average is equal to $9.5 \mathrm{kpc}$. López-Corredoira et al. (2002) results show a variation of the scale height due to the flare given by:

$$
h_{Z}(R)=h_{Z}\left(R_{\odot}\right) e^{\frac{R-R_{\odot}}{(12-0.6 R)}}
$$

for $\mathrm{R}<15 \mathrm{kpc}$ where $\mathrm{R}$ is given in $\mathrm{kpc}, R_{\odot}=7.9 \mathrm{kpc}$ and $h_{Z}\left(R_{\odot}\right)=285 \pm 12 \mathrm{pc}$. 
Reylé et al. (2009) fitted the 2MASS survey with simulations from the Besançon Galaxy Model, which model the flare as Gyuk et al. (1999) (See section 3.2.2).

$$
K_{\text {flare }}(R)=1+\gamma_{\text {flare }}\left(R-R_{\text {flare }}\right)
$$

where $\gamma_{\text {flare }}$ is the amplitude of the flare, $K_{\text {flare }}$ is the factor to increase the scale heights and $R_{\text {flare }}$ is the galactocentric distance where the flare starts. The amplitude is assumed equal to $5.4 \times 10^{-5} \mathrm{pc}^{-1}$ and the starting radius is in the Sun position. In section 3.2.2 we will describe, how it is implemented in the Besançon Galaxy Model.

\subsection{Stellar halo}

The halo is thought to be the oldest (age $\geq 13 \mathrm{Gyr}$ ), and most extended component of the galaxies. The age of the halo has been obtained in different analysis. Sneden et al. (1996) obtained an age of 15.2 $\pm 3.7 \mathrm{Gyr}$, while Cayrel et al. (2001) obtained $14 \pm 3 \mathrm{Gyr}$ and Frebel et al. (2007) derived 13.2 Gyr. Nevertheless, more recently, ages lower that 13 Gyr were obtained in De Angeli et al. (2005) and Hansen et al. (2013) for globular clusters and Kalirai (2012) for white dwarfs. A compilation of 41 globular clusters studied by Schiavon et al. (2005) is made in Roediger et al. (2014) who found a strong peak in the age distribution at 12.5-13.0 Gyr.

Carollo et al. (2007, 2010, 2014) claimed that the stellar haloes can have two different components. One inner component which dominates at shorter distances from the galactic center, in the range from $10 \mathrm{kpc}$ to $15 \mathrm{kpc}$, flatter $(q \sim 0.6)$, with slightly higher orbital eccentricities, a smaller prograde rotation (between 0 and $50 \mathrm{~km} \mathrm{~s}^{-1}$ ) and a metallicity distribution that peaks at -1.6 dex. One outer halo which begins to dominate at larger distances from the galactic center between 15 and $20 \mathrm{kpc}$, which is more spherical $(q \sim 0.9-1.0)$, covering a wide range of orbital eccentricities, from circular to very eccentric, and a retrograde rotation (between -40 and -70 $\mathrm{km} \mathrm{s}^{-1}$ ) (Carollo et al. 2010; de Jong et al. 2010; Beers et al. 2012). The metallicity distribution peaks at -2.2 dex with a strong lower metallicity tail. This scenario is contested by Schönrich et al. (2011) which stated that if there is an outer halo component it has to be less strong than predicted by the above cited works. More recently Robin et al. (2014) showed that if there is a inner-outer halo transition it cannot be at smaller distances than $30 \mathrm{kpc}$. Assuming a density which has a power-law shape a value of $q=0.7$ is obtained and if an Hernquist shape is assumed the value of the axis ratio becomes equal to 0.77 . The stellar halo has a total mass around $1 \times 10^{9} M_{\odot}$ (Carney et al. 1990) inside a dark matter halo of around $1 \times 10^{12} M_{\odot}$ (Finkbeiner 2012).

The stellar halo extension in the Milky Way is still unknown but old bright stars like RR Lyrae and blue horizontal branch stars (BHB) have been used as tracers of the halo component. They reach distances of $115 \mathrm{kpc}$ from the galactic center (Clewley et al. 2005) and beyond. The dark halo is more extended and its half-mass radius is of $150 \mathrm{kpc}$ (Klypin et al. 2002; Battaglia et al. 2005, 2006). Complete reviews of the halo component can be found in Freeman \& Bland-Hawthorn (2002) and Helmi (2008). 


\subsection{Bulge}

The bulge region contains a sum of different components among which :

- The inner disc

- The bar

- The inner part of the thick disc

- Possibly a small classical bulge (under discussion)

The bulge stars have ages around $10 \pm 2.5$ Gyr (Ortolani et al. 1995; Zoccali et al. 2003). The structure is like a boxy bulge with a possible orientation of 13-45 degrees with the nearest side located at positive longitudes (Rattenbury et al. 2007). Different analysis obtained lower values as 20 degrees (e.g. Freudenreich 1998; Bissantz \& Gerhard 2002, among others) used in Pichardo et al. (2004) models or 13 degrees using the Besançon Galaxy Model (Robin et al. 2012). More recent analysis towards the bulge region claim that the Milky Way bulge is Xshaped (McWilliam \& Zoccali 2010; Zoccali 2010; Nataf et al. 2010; Saito et al. 2011).

The metallicity distribution is asymmetric in the range of -1.5 dex to 0.5 dex and with a mean of -0.26 dex but results in these regions may be largely contaminated by other components like the inner disc, the bar or the thick disc (Zoccali et al. 2008). So, the MDF of the bulge was found to be a more complex structure which can have from two (e.g. Babusiaux et al. 2010; Hill et al. 2011; Rojas-Arriagada et al. 2014) up to five components (Ness et al. 2013). The bulge is rotating with a peak at $75 \mathrm{~km} \mathrm{~s}^{-1}$ (e.g. Minniti et al. 1992; Ibata \& Gilmore 1995; Minniti 1996; Rich et al. 2007, among others) and a large velocity dispersion (Terndrup et al. 1995; Ibata \& Gilmore 1995; Minniti 1996) that decreases with Galactocentric distance. Figure 2.14, taken from Minniti \& Zoccali (2008) shows the velocity dispersion and mean velocity as a function of the galactic longitude.

The total mass has been calculated in some works which obtained $\sim 1.0 \times 10^{10} M_{\odot}$ (Matsumoto et al. 1982; Kent 1992), or larger values around $1.6 \times 10^{10} M_{\odot}$ (Allen \& Santillan 1991; Dwek et al. 1995; Bissantz \& Gerhard 2002; Gerhard 2006; Sumi et al. 2006; Sofue et al. 2009), around $2.0 \times 10^{10} M_{\odot}$ (Zhao \& Mao 1996; Wang et al. 2012). Weiner \& Sellwood (1999) obtained a bar mass of $9.8 \times 10^{9} M_{\odot}$ and a bulge mass of $5.4 \times 10^{9} M_{\odot}$ which gives a total mass of $1.52 \times 10^{10} M_{\odot}, 5.18 \times 10^{9} M_{\odot}$ (Binney \& Tremaine 2008b) and $6.1 \times 10^{9} M_{\odot}$ for the sum of two components (Robin et al. 2012).

The bulge formation is still under debate and is possible that Milky Way mass galaxies having no classical bulges are common in the nearby Universe. (Laurikainen et al. 2014). Simmons et al. (2014) shows that bars are present in $25 \%-50 \%$ of the locally analysed disc galaxies. There are three scenarios that are invoked. (1) in situ formation due to gas collapse from a protogalactic gas cloud (Eggen et al. 1962; Samland \& Gerhard 2003). (2) The $\Lambda$ CDM, a hierarchical scenario, where the accretion of substructures like satellites or Galaxy mergers drives the growth 

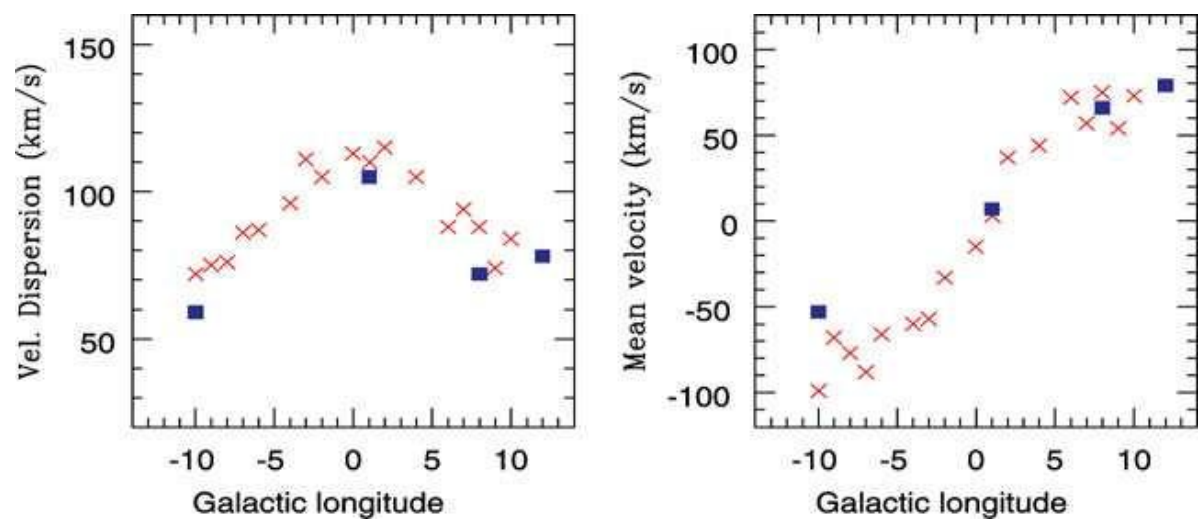

Figure 2.14: Figure from Minniti \& Zoccali (2008). The velocity dispersion and mean velocity as a function of the galactic longitude in the bulge component. Blue squares are $\mathrm{K}$ giants measured by Minniti (1996) and red crosses are M-giants measured by Rich et al. (2007) and which were corrected for the solar motion around the Galaxy.

of a bulge (Aguerri et al. 2001; Scannapieco \& Tissera 2003; Immeli et al. 2004) (See section 2.2). (3) Secular formation due to the bar-buckling instability and not mergers which drive the growth of a boxy bulge (Saha \& Gerhard 2013). In early times the bar can be formed in a secular process related to disc instability which redistributes energy and angular momentum between the disc, dark matter halo and classical bulge in a more efficient way (Combes \& Sanders 1981; Pfenniger \& Norman 1990; Raha et al. 1991; Debattista \& Sellwood 2000; Athanassoula 2003; Martinez-Valpuesta \& Shlosman 2004; Kormendy \& Kennicutt 2004; Debattista et al. 2006; Saha et al. 2012). The boxy bulges as the one observed in the Milky Way are believed to be formed by bar-buckling instability as the bar goes stronger. The different scenarios can co-exist to explain the formation and evolution of the bulge. 


\section{Chapter 3}

\section{The Besançon Galaxy Model}

\subsection{Introduction}

For this analysis we use the Besançon Galaxy Model (hereafter BGM) to produce simulations. The model aims at producing simulations, compare with observations and test different scenarios of Galaxy formation and evolution. The BGM has been constructed and developed in a series of published releases since Robin \& Crézé (1986) until the last improvement made in Robin et al. (2014). The model includes the bar and spiral arms are not modelled, in the current version (the spiral structure is under study). The model gathers dynamical constrains, models of stellar atmospheres and theories of stellar formation and evolution where the link between the Galactic evolution and dynamics is given by the stellar ages. The main idea is to start with a series of assumptions, our a priori knowledge, simulate the Galaxy, compare it with a large number of observational data in a wide range of Galactic longitudes and latitudes and constrain several parameters involved in the BGM construction. We can use this tool to compare with photometric, astrometric and spectroscopic data to provide better constrains about the structure and dynamics of our Galaxy and to understand better the formation and evolution of the Milky Way. We use a population synthesis approach which takes into account biases by simulating observational errors and by comparing the observations with simulations in the space of observables.

The version used for the BGM assumes that stars belong to 4 different populations: the thin disc, the thick disc, the stellar halo and the bulge/bar. Furthermore, the thin disc is divided in seven different sub-populations of different ages. Each of these populations is characterized by an initial mass function (IMF), a star formation rate (SFR), density laws, kinematics, agemetallicity relation and chemical distributions. Separately, the white dwarfs population is taken into account and it is included in the dynamical considerations. The density laws (in particular the scale heights) are constrained (see section 3.2.4) through the Boltzmann equation, by using the potential of the Galaxy and assuming an empirical age-velocity dispersion relation (Bienaymé et al. 1987). In the following subsections we describe the BGM in more details. 


\subsection{The overall structure}

The thin disc is assumed to be divided in seven different subpopulations according to their ages has shown in table 3.1. The warp and flare structures included are discussed in Reylé et al. (2009) and will be presented in section 3.2.2. To compute the number of stars, in the Milky Way, for a given direction the model uses the fundamental equation of stellar statistics

$$
A(m)=\sum_{i=M_{v_{\min }}}^{M_{v_{\max }}} \int_{0}^{r} \phi(M) \rho(r) r^{2} \omega d r
$$

where $A(m)$ is the number of stars with apparent magnitude $\mathrm{m}$ inside a solid angle $\omega, \mathbf{M}$ is the absolute magnitude and $\mathrm{r}$ is the heliocentric distance. Equation 3.1 is the product between the luminosity function $(\phi(M))$ and the density law $(\rho(r))$. For each component (halo, bulge, thin and thick disc), are assumed different luminosity functions and density laws. In Galactic coordinates one obtain

$A(m)=\sum_{i=1}^{4} \sum_{a=M_{v_{\min }}}^{M_{v_{\max }}} \sum_{b=T_{e f f_{\min }}}^{T_{\text {effmax }}} \sum_{c=a g e_{\min }}^{\text {age }_{\max }} \int_{0}^{r} \int_{b_{\min }}^{b_{\max }} \int_{l_{\min }}^{l_{\max }} \phi_{i}\left(M_{v}, T_{\text {eff }}\right.$, age $) \rho_{i}(R, \theta, Z$, age $) r^{2} \cos (b) d l d b d r$

$\phi_{i}\left(M_{v}, T_{e f f}, a g e\right)$ refers now to the "Hess diagram" which is the density of stars (stars $\left.\mathrm{pc}^{-3}\right)$ in a point of the 3 dimensional space given by the absolute magnitude, effective temperature and age. It defines the stellar content in the solar neighbourhood. It has been computed from the stellar evolution model described in Haywood et al. (1997a,b). One can extend this information outside the solar neighbourhood. The subindex i refers to the Galactic population, $M_{v}$ is the absolute magnitude in band $\mathrm{V}, T_{\text {eff }}$ is the effective temperature, $\mathrm{R}$ is the galactocentric distance and $\mathrm{Z}$ is the height from the plane. The overall structure of the algorithm is presented in the organigram shown in figure 3.1 (Robin \& Crézé 1986).

\subsubsection{The luminosity function and Hess diagram}

The luminosity function gives the number of stars by magnitude interval. The mass distribution, for one specific epoch is given by the Initial Mass Function (IMF) defines the way the stars are distributed in the H-R diagram represented in a "Hess diagram" constructed based on the IMF, SFR and evolutionary tracks sets fixed by fitting to the observational data (Haywood et al. 1997b). The IMF and the SFR for each component are given in table 3.1. The SFR was defined to be constant and a two slope IMF was defined for the thin disc as $\propto m^{\alpha}$ where $\alpha=-(1+x)=$ -1.6 for stars with masses lower than $1 M_{\odot}$ and $\alpha=-3$ otherwise.

The thick disc was assumed to have a single epoch of star formation (The thick disc formation period is short in comparison with the age of the Galaxy), and an age of 12 Gyr. The IMF for the thick disc was assumed to be $\propto m^{-1.5}$. 


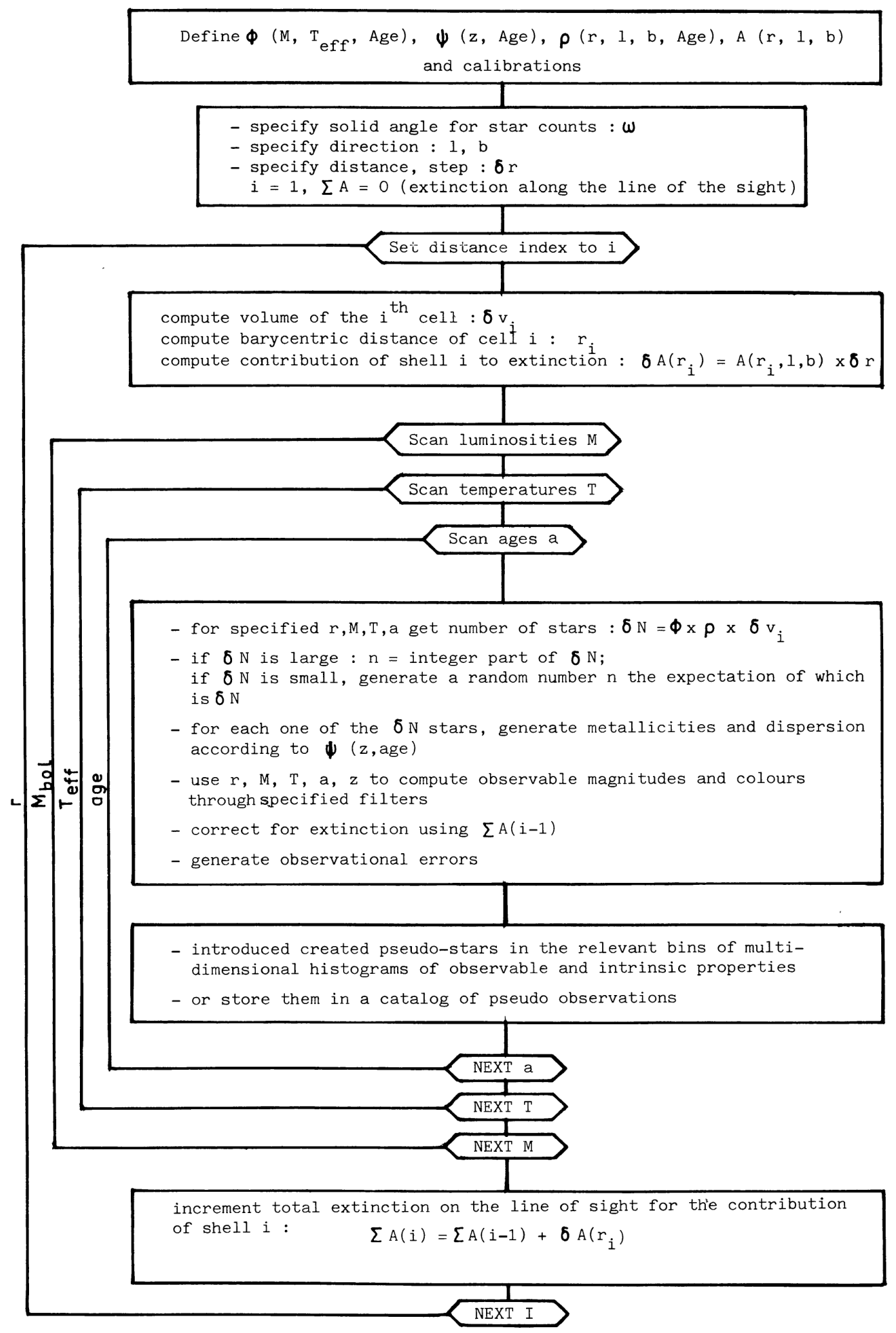

Figure 3.1: Figure 1 of Robin \& Crézé (1986). Flowchart of the algorithm. 
Table 3.1:

Table 1 from Robin et al. (2003). Defined initial mass function (IMF) and star formation rate (SFR) for all the model components

\begin{tabular}{lccc}
\hline \hline Pop & Age (Gyr) & IMF $(\alpha)$ & SFR \\
\hline & $0-0.15$ & & \\
& $0.15-1.0$ & & \\
& $1.0-2.0$ & $\frac{d n}{d m} \propto m^{\alpha}$ & \\
Thin disc & $2.0-3.0$ & $\alpha=-1.6$ for $m<1 M_{\odot}$ & constant \\
& $3.0-5.0$ & $\alpha=-3.0$ for $m>1 M_{\odot}$ & \\
& $5.0-7.0$ & & \\
\hline Thick disc & $7.0-10.0$ & & one burst \\
\hline Halo & 12.0 & $\frac{d n}{d m} \propto m^{-1.5}$ & one burst \\
\hline \hline
\end{tabular}

The halo also has one single epoch of star formation, an age of 14 Gyr and the IMF of the halo is assumed to be $\propto m^{-1.5}$.

\subsubsection{Density laws}

The density distribution is constrained by assuming density laws, suitable for each Galactic component, and by combining this information with the local density. The total local mass density is the sum of the different mass components in the Milky Way (thin and thick disc and stellar halo), the interstellar medium (ISM) and the dark matter halo. The local mass density in stars value is around $0.045 M_{\odot} \mathrm{pc}^{-3}$ (Wielen 1974a) but Jahreiß \& Wielen (1997) found 0.039 $M_{\odot} \mathrm{pc}^{-3}$. For main sequence stars only Reid et al. (2002) cite a value of $0.033 M_{\odot} \mathrm{pc}^{-3}$. The ISM contributes with $0.021 M_{\odot} \mathrm{pc}^{-3}$ (Dame 1993). Adding the local mass density from stars with the interstellar medium and the local contribution of the dark matter Robin et al. (2003) obtained values in agreement with the total mass density determined dynamically (Crézé et al. 1998).

The thin disc density law is sliced into two density laws, with different scale length, based on the age of the population. The density laws are simple Einasto profiles with parameters defined as a scale length $h_{r_{+}}$, a hole scale length $h_{r_{-}}$and $\epsilon$ which is the eccentricity of the ellipsoid.

The model assumes, for the thick disc, a shape for the density law which decomposes vertically into two different mathematical laws according to the distance from the plane. A parabola at short distances, followed by an exponential at larger distances (Equation 3.3). 


$$
\rho(R, Z)=\left\{\begin{array}{l}
\rho_{0} \times \exp \left(-\frac{R-R_{\odot}}{h_{R}}\right) \times\left(1-\frac{\frac{1}{h_{Z}}}{\xi \times\left(2+\frac{\xi}{h_{Z}}\right)} \times Z^{2}\right) \text { if }|Z| \leq \xi \\
\rho_{0} \times \exp \left(-\frac{R-R_{\odot}}{h_{R}}\right) \times \exp \left(-\frac{\left|Z-Z_{\odot}\right|}{h_{Z}}\right) \text { if }|Z|>\xi
\end{array}\right.
$$

where $\rho_{0}$ is the local density at the solar position (The distance of the Sun to the Galactic center is assumed to be $8.0 \mathrm{kpc}$ (Reid 1993; Brunthaler et al. 2011) and the assumed distance to the Galactic plane is $Z=15$ pc) (Hammersley et al. 1995; Freudenreich 1998; Drimmel \& Spergel 2001), $h_{R}$ the scale length, $h_{Z}$ the scale height and $\xi$ is the distance from the plane at which the density law changes. The scale length of the thick disc is 2355 pc (Robin et al. 2014) and the scale height is $533 \mathrm{pc}$.

\section{Warp in the BGM}

The warp has been included in the BGM (Robin et al. 2003) as a S-shape warp tilted ring model (Porcel et al. 1997). The authors computed the height $Z_{\text {warp }}$ above the plane $b=0^{\circ}$ as a function of the cylindrical coordinates.

$$
Z_{\text {warp }}(R)=\gamma_{\text {warp }}\left(R-R_{\text {warp }}\right) \times \sin \left(\phi-\phi_{\text {warp }}\right) \text { if } R \geq R_{\text {warp }}
$$

where $Z_{\text {warp }}$ is the distance in $\mathrm{Z}$ of the mid-plane to the plane defined by $\mathrm{b}=0^{\circ}, \gamma_{\text {warp }}$ is the slope of the warp,$R_{\text {warp }}$ the galactocentric distance where the warp starts, $\phi$ is the Galactic azimuth and $\phi_{\text {warp }}$ is the angle where the warp is maximum. Robin et al. (2003) followed Burton (1988) which assumed, as other works, that the Sun lies on the line of the nodes of the warp $\left(\phi_{\text {warp }}=0^{\circ}\right)$. The authors used $R_{\text {warp }}=8.4 \mathrm{kpc}$ (Derriere \& Robin 2001) and an amplitude of $\gamma_{\text {warp }}=0.18$ in agreement with Gyuk et al. (1999). More recently Reylé et al. (2009) revised the parameters of the warp testing different shapes and parameters for the warp. The authors assumed $R_{\text {warp }}=8.4 \mathrm{kpc}$ has a best value (Derriere $\&$ Robin 2001), and tried two different sets of values for the slope of the warp and for the scale length. The two sets are $\left(\gamma_{\text {warp }}, h_{R}\right)=(0.18$, $2530)$ and $\left(\gamma_{\text {warp }}, h_{R}\right)=(0.09,2200)$. $\phi_{\text {warp }}$ is assumed to be equal to zero. It was also tried to fit a two Fourier-mode warp model from Levine et al. (2006) with their best set of parameters. The best fit has been achieved with a linear increase of the warp with the following set of parameters: $\left(\gamma_{\text {warp }}, h_{R}\right)=(0.09,2200)$, but the authors noticed that while the fit was satisfactory at positive longitudes, it was not the case for negative longitudes, where the warp appears not symmetrical with regard to the other side of the Milky Way.

\section{Flare in the BGM}

In the BGM the flare is modeled as in Gyuk et al. (1999) by increasing the scale height by a $k_{\text {flare }}$ factor beyond the galactocentric radius $R_{\text {flare }}$ where the flare starts.

$$
K_{\text {flare }}(R)=1+\gamma_{\text {flare }}\left(R-R_{\text {flare }}\right) \text { if } R \geq R_{\text {flare }}
$$


3. The Besançon Galaxy Model

Table 3.2:

Table 4 from Robin et al. (2003). Age-velocity relations for the different components of the model.

\begin{tabular}{lcccccc}
\hline \hline Pop & $\begin{array}{c}\text { Age } \\
(\mathrm{Gyr})\end{array}$ & $\begin{array}{c}\sigma_{U} \\
\left(\mathrm{Km}^{-1}\right)\end{array}$ & $\begin{array}{c}\sigma_{V} \\
\left(\mathrm{Km}^{-1}\right)\end{array}$ & $\begin{array}{c}\sigma_{W} \\
\left(\mathrm{Km}^{-1}\right)\end{array}$ & $\begin{array}{c}V_{a d} \\
\left(\mathrm{Km} \mathrm{s}^{-1}\right)\end{array}$ & $\begin{array}{c}d \sigma_{U} / d R \\
\left(\mathrm{Km} \mathrm{s}^{-1} \mathrm{Kpc}^{-1}\right)\end{array}$ \\
\hline \multirow{4}{*}{ Thin disc } & $0-0.15$ & 16.7 & 10.8 & 6 & 3.5 & \\
& $0.15-1.0$ & 19.8 & 12.8 & 8 & 3.1 & \\
& $1.0-2.0$ & 27.2 & 17.6 & 10 & 5.8 & -0.2 \\
& $2.0-3.0$ & 19.5 & 10.8 & 13.2 & 7.3 & \\
& $5.0-5.0$ & 36.7 & 23.7 & 15.8 & 10.8 & 0 \\
\hline Thick disc & $7.0-10.0$ & 43.1 & 27.8 & 17.4 & 14.8 & \\
\hline \hline
\end{tabular}

where $\gamma_{\text {flare }}$ is the slope of the flare and $R_{\text {flare }}$ is the galactocentric distance where the flare starts. In the BGM (version Robin et al. (2003)) the starting position of the flare is assumed to be $9.5 \mathrm{kpc}$ following (Derriere \& Robin 2001) and the factor that modifies the scale heights is assumed to be $5.4 \times 10^{-4} \mathrm{kpc}^{-1}$ from the Hubble Space Telescope (HST) observations (Gyuk et al. 1999). A shorter $R_{\text {flare }}$ of $8.4 \mathrm{kpc}$, proposed in Derriere \& Robin (2001), has been tested in Reylé et al. (2009) but without improvements in the comparison between observations and simulations.

\subsubsection{Model kinematics}

The age-velocity dispersion relation used for the thin disc has been deduced from Hipparcos data (Gomez et al. 1997). In table 3.2 we show the age-velocity relations for the thin and thick disc. In the model N-body simulations are used to define the kinematics of the stars in the bar at different positions. The model uses alternatively Fux (1997, 1999) or Debattista (2006) simulations R1 and B3 (according to Gardner et al 2013). In one of the possible applications Gardner et al. (2014) used the BGM to study the X-shape of the Milky Way's bulge against BRAVA observations (Bulge Radial Velocity Assay, Kunder et al. 2012) obtaining a best fit for a bar angle of $15^{\circ}$. The Sun velocities are $U_{\odot}=11.0 \mathrm{Km} \mathrm{s}^{-1}, V_{\odot}=12.0 \mathrm{Km} \mathrm{s}^{-1}, W_{\odot}=7.0 \mathrm{Km}$ $s^{-1}$ and $V_{L S R}=226.5 \mathrm{Km} \mathrm{s}^{-1}$, with $\mathrm{U}$ positive in the direction of the Galactic center, $\mathrm{V}$ positive in the direction of Galactic rotation, and $\mathrm{W}$ positive in the direction of the North Galactic Pole. 


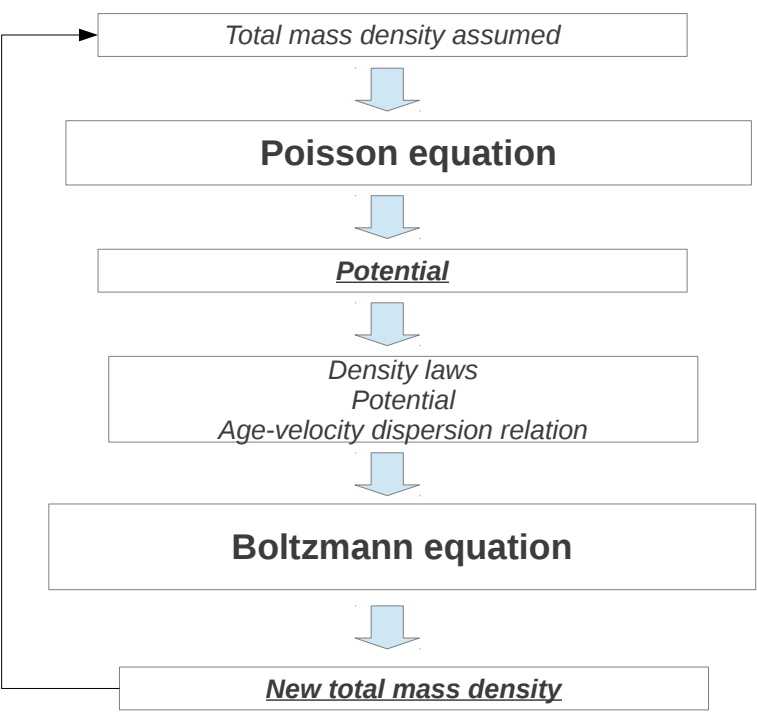

Figure 3.2: Scheme of the algorithm to compute the dynamical self-consistency. Ingredients for the equations are shown in italic, equations are shown in bold and results from equations are underlined.

\subsubsection{The dynamical self-consistency}

The originality of the BGM, in comparison with other population synthesis models is the dynamical self-consistency (Bienaymé et al. 1987). One can compute the potential using Poisson's equation by adding up the densities of the four different populations in addition to the dark matter halo and the interstellar medium. If one assumes that all sub-populations of the thin disc, less the younger one, are isothermal and relaxed the individual velocity dispersion is set up by the age-velocity dispersion. One can constrain the scale height from the Galactic potential and from the velocity dispersion using the Boltzmann equation. From the density laws one can compute a new mass density and a new potential by Poisson's equation. The process is iterated until the Galactic potential and scale heights converge (change less than 1\%). Figure 3.2 shows the scheme used to compute the dynamical self-consistency.

\subsection{The metallicity distribution}

For the thin disc an age-metallicity relation according to Haywood (2006b) is assumed (table 3.3). We assume gradients and a gaussian distribution centered on the mean metallicity and a dispersion for each age bin. For the thick disc a mean metallicity of -0.7 dex with a dispersion of 0.3 dex around this value (Bensby et al. 2007; Fuhrmann 2011), and no radial and vertical 
gradient are assumed. In the thick disc and halo components the distribution of stars is computed from alpha-enhanced isochrones (Bergbusch \& Vandenberg 1992) for an age range from 10 Gyr to $12 \mathrm{Gyr}$ in the standard model. The halo metallicity was assumed to be -1.78 dex with a dispersion of 0.5 dex (Ivezić et al. 2008), with no gradient, and simulated using same $\alpha$ enhanced isochrones. Table 3.3 summarizes the metallicity distribution for each population used in the simulations, before fitting.

Table 3.3:

Table 1 from Robin et al. (2003). Metallicity distributions assumed in original simulations: Age, mean metallicity, radial metallicity gradient and dispersion for each population.

\begin{tabular}{lcccc}
\hline \hline Pop & $\begin{array}{c}\text { Age } \\
(\mathrm{Gyr})\end{array}$ & $\begin{array}{c}{[\mathrm{Fe} / \mathrm{H}]_{\odot}} \\
(\mathrm{dex})\end{array}$ & $\begin{array}{c}\frac{d[\mathrm{Fe} / \mathrm{H}]}{d R} \\
(\mathrm{dex} / \mathrm{kpc})\end{array}$ & $\begin{array}{c}\text { Dispersion } \\
(\mathrm{dex})\end{array}$ \\
\hline \multirow{4}{*}{ Thin disc } & $0-0.15$ & 0.01 & & 0.10 \\
& $0.15-1.0$ & 0.00 & & 0.11 \\
& $1.0-2.0$ & -0.02 & & 0.12 \\
& $3.0-3.0$ & -0.03 & -0.07 & 0.13 \\
& $5.0-7.0$ & -0.05 & & 0.14 \\
& $7.0-10.0$ & -0.09 & & 0.16 \\
\hline Thick disc & 12.0 & -0.78 & & 0.18 \\
\hline Halo & 14.0 & -1.78 & 0.00 & 0.30 \\
\hline \hline
\end{tabular}

\subsection{A revised model for the thin disc}

In this analysis we have used two revised versions (model A and model B, where A and B refer to the alternative models of the BGM (Czekaj et al. 2014) along with the standard version. The main changes made in this work are the inclusion of binarity from Arenou (2011), new evolutionary tracks, new atmosphere models, new age-metallicity relation, new dynamical mass, and the use of different IMF and SFR. The SFR in the standard model was assumed to be constant, but in the new version it is assumed to be decreasing with time, following Aumer \& Binney (2009) prescription. Czekaj et al. (2014) tried various IMF, combining different slopes in different mass ranges. They conclude that Tycho data favor two kind of IMF, a so-called HaywoodRobin for Model A, well in agreement with data in the place, and Kroupa-Haywood(V6) for model B, well suited for higher latitudes. The two kind of IMF are given in Table 1.4 (see their paper for more details). They will be tested alternatively with the standard model in our analysis. 
Table 3.4:

SFR and IMF parameters in the standard and revised versions of the BGM.

\begin{tabular}{lcc}
\hline \hline Model & SFR & IMF $(\alpha)$ \\
\hline \multirow{2}{*}{ Model Standard } & Constant & 1.6 for $M \leq 1 M_{\odot}$ \\
& & 3.0 for $M>1 M_{\odot}$ \\
\hline \multirow{2}{*}{ Model A } & Decreasing & 1.6 for $M \leq 1 M_{\odot}$ \\
& & 3.0 for $M>1 M_{\odot}$ \\
\hline \multirow{2}{*}{ Model B } & Decreasing & 1.3 for $0.09 M_{\odot}<M<0.5 M_{\odot}$ \\
& & 3.2 for $0.5 M_{\odot}<M<1.53 M_{\odot}<M<120 M_{\odot}$ \\
\hline \hline
\end{tabular}

\subsubsection{Atmosphere models}

The atmosphere models used in the standard model are the BaSeL 2.2 library from Lejeune et al. (1998). In the new model one can choose between BaSel 2.2 and BaSeL 3.1 atmosphere models. The BaSel 2.2 stellar library is the compilation of different atmospheric models (Kurucz (1995), Bessell et al. (1989), Bessell et al. (1989), Fluks et al. (1994), Allard \& Hauschildt (1995) for M dwarfs). The BaSel 2.2 was only calibrated from solar metallicity data which implied the presence of some problems at low metallicities $([\mathrm{Fe} / \mathrm{H}]<-1.0 \mathrm{dex})$. This weakness has been corrected in Westera et al. (2002) and following this correction it was created the new library BaSeL 3.1 used in model A and B.

\subsubsection{Evolutionary tracks}

The evolutionary tracks used in the standard model (see Haywood et al. (1997a) for a more detailed description) have been revised for model A and B. In the low mass regime the standard model uses Vandenberg (private communication) whereas the new versions use Chabrier \& Baraffe (1997). For higher masses the standard model uses Schaller et al. (1992) tracks whereas the new versions use Bertelli et al. $(2008,2009)$ and in model B Bertelli et al. (1994) for masses larger than 20 solar masses. Table 3.5 shows a comparison between the versions. See Czekaj et al. (2014) for a more detailed description. 
Table 3.5:

Table 2 of Czekaj et al. (2014). Evolutionary tracks for the standard and revised versions of the BGM

\begin{tabular}{lc}
\hline \hline Model & evolutionary tracks references \\
\hline \multirow{3}{*}{ Model Standard } & Vandenberg (private communication) for $\mathrm{M}<1 M_{\odot}$ \\
& Schaller et al. (1992) for $\mathrm{M}>1 M_{\odot}$ \\
& Castellani et al. (1992) for helium-burning stars at masses $1-1.7 M_{\odot}$ \\
\hline Model A & Chabrier \& Baraffe (1997) for $\mathrm{M}<0.6 M_{\odot}$ \\
& Bertelli et al. (1994) for $\mathrm{M}>0.6 M_{\odot}$ \\
\hline \multirow{2}{*}{ Model B } & Chabrier \& Baraffe (1997) for $\mathrm{M}<0.7 M_{\odot}$ \\
& Bertelli et al. (2008, 2009) for $0.7 M_{\odot}<\mathrm{M}<20 M_{\odot}$ \\
\hline \hline
\end{tabular}

\subsubsection{Binarity}

The binarity is included in the revised version implementing the scheme used in Arenou (2011) where the probability of the existence of a binary star is related to the mass and luminosity class of the primary object. If the star belongs to the main sequence the probability depends on the mass of the primary star

$$
p(M)=0.85 \times \tanh (0.55 M+0.095)
$$

where $\mathrm{M}$ is the mass of the object. If the primary star is a giant it has $60 \%$ of being a binary. The separation of the system depends on the luminosity class (Arenou 2011).

\subsubsection{Age-metallicity relation}

The age-metallicity relation also suffered a modification from the standard to the revised model. In the revised version we use the age-metallicity relation from Haywood (2006a). The scheme to assign the metallicity to stars has changed. In the revised model metallicity and dispersion are now computed following equations 3.7 and 3.8 where the equations are a fit to Haywood (2006b) values from the GCS (Nordström et al. 2004) data.

$$
\begin{gathered}
{[\mathrm{Fe} / \mathrm{H}]=-0.077 \times \text { age }+0.156} \\
\sigma_{[\mathrm{Fe} / \mathrm{H}]}=0.010 \times \text { age }+0.1192
\end{gathered}
$$


Table 3.6:

Table 5 from Czekaj et al. (2014). Ingredients of the standard and revised simulations

\begin{tabular}{|c|c|c|c|}
\hline Ingredients & Standard model & Model A & Model B \\
\hline SFR & constant & $\begin{array}{l}\text { decreasing } \exp (-0.12 \tau) \\
\text { Aumer \& Binney }(2009)\end{array}$ & $\begin{array}{l}\text { decreasing } \exp (-0.12 \tau) \\
\text { Aumer \& Binney }(2009)\end{array}$ \\
\hline IMF & Haywood-Robin & Haywood-Robin & Haywood-Robin v6 \\
\hline Age-metallicity relation & Twarog (1980) & Haywood (2006a) & \\
\hline atmosphere models & BaSeL 2.2 & BaSeL 3.1 & BaSeL 3.1 \\
\hline binarity & no & $\begin{array}{l}\text { yes - scheme adopted } \\
\text { from Arenou (2011) }\end{array}$ & $\begin{array}{l}\text { yes - scheme adopted } \\
\text { from Arenou (2011) }\end{array}$ \\
\hline age of the thin disc & $10 \mathrm{Gyr}$ & $10 \mathrm{Gyr}$ & $10 \mathrm{Gyr}$ \\
\hline thick disc parameters & $\begin{array}{c}h_{R}=2355 \mathrm{pc} \\
h_{Z}=533 \mathrm{pc} \\
\text { density }=8.33 \times 10^{-3} * / \mathrm{pc}^{3}\end{array}$ & $\begin{array}{c}h_{R}=2355 \mathrm{pc} \\
h_{Z}=533 \mathrm{pc} \\
\text { density }=8.33 \times 10^{-3} * / \mathrm{pc}^{3}\end{array}$ & $\begin{array}{c}h_{R}=2355 \mathrm{pc} \\
h_{Z}=533 \mathrm{pc} \\
\text { density }=8.33 \times 10^{-3} * / \mathrm{pc}^{3}\end{array}$ \\
\hline extinction model & Drimmel \& Spergel (2001) & $\begin{array}{c}\text { Marshall et al. (2006) + } \\
\text { Drimmel \& Spergel (2001) }\end{array}$ & $\begin{array}{c}\text { Marshall et al. (2006) + } \\
\text { Drimmel \& Spergel (2001) }\end{array}$ \\
\hline total dynamicall mass & Crézé et al. (1998) & van Leeuwen (2007) & van Leeuwen (2007) \\
\hline local stellar mass density & Wielen (1974b) & Wielen (1974b) & Jahreiß \& Wielen (1997) \\
\hline local stellar mass density & Robin et al. (2003) & Binney \& Tremaine (2008b) & Binney \& Tremaine (2008b) \\
\hline Age-velocity relation & Gomez et al. (1997) & Gomez et al. (1997) & Gomez et al. (1997) \\
\hline Warp and flare & Reylé et al. (2009) & Reylé et al. (2009) & Reylé et al. (2009) \\
\hline scale length & $\begin{array}{c}\text { young disc } h_{R}=5000.0 \mathrm{pc} \\
\text { old disc } h_{R}=2400.0 \mathrm{pc}\end{array}$ & $\begin{array}{l}\text { young disc } h_{R}=5000.0 \mathrm{pc} \\
\text { old disc } h_{R}=2400.0 \mathrm{pc}\end{array}$ & $\begin{array}{c}\text { young disc } h_{R}=5000.0 \mathrm{pc} \\
\text { old disc } h_{R}=2400.0 \mathrm{pc}\end{array}$ \\
\hline
\end{tabular}

\subsubsection{Dynamical mass}

The dynamical mass has also been revised in the new configuration. The original one was Crézé et al. (1998), but van Leeuwen (2007) computed a new reduction of the Hipparcos data that leads to a new total dynamical mass of $(0.122 \pm 0.019) M_{\odot} \mathrm{pc}^{-3}$ that is $60 \%$ higher than the one assumed by Crézé et al. (1998) of $(0.076 \pm 0.015) M_{\odot} \mathrm{pc}^{-3}$. In order to use the new dynamical mass the interstellar medium mass density has been increased to the value proposed by Binney \& Tremaine (2008b) of $0.05 M_{\odot} \mathrm{pc}^{-3}$.

The following table 3.6 based in the table 5 of Czekaj et al. (2014) gives a summary of the main changes introduced in the revised models in comparison with the standard model. 


\subsection{A new thick disc in the BGM}

So far the BGM have assumed a single epoch formation for the thick disc but several recent results (Bovy et al. 2012b; Haywood et al. 2013, among others) claim a longer thick disc formation period (4-5 Gyr). In Robin et al. (2014) new thick disc parameters have been tested and fitted to photometric data (SDSS and 2MASS). The thick disc was tentatively divided into two parts of different ages in order to check for a longer epoch of star formation. This implementation results in a better fit than the original single thick disc epoch formation. The older part of the thick disc has $12 \mathrm{Gyr}$, a scale length of about $3 \mathrm{kpc}$ and a scale height of $800 \mathrm{pc}$ (assuming a $\mathrm{sech}^{2}$ ). The younger part has an age of $10 \mathrm{Gyr}$, a scale length of $2 \mathrm{kpc}$ and a scale height of about $350 \mathrm{pc}$. The young thick disc metallicity distribution was assumed to have a mean metallicity of -0.5 dex in agreement with recent works from the literature presented in Table 8.14 and from the SEGUE analysis presented further in this work. The mean metallicity of the old thick disc was assumed to be equal to -0.78 dex in agreement with the values used in the description of the BGM (See section 3.3). Both components of the thick disc were assumed to have a dispersion of 0.30 dex and no radial or vertical metallicity gradients. In section 9 we discuss the results of the fit of this version of the model with Gaia-ESO survey data. 


\section{Chapter 4}

\section{Surveys}

\subsection{Introduction}

Spectroscopic surveys, in last decade, evolved from the study of a few hundred of stars to the analysis of large samples of stars for which one can compute distributions and trace properties of populations. Looking at the present and future observational projected surveys we see that there is a golden era for Galactic surveys data mining. The first large spectorcopic survey which showed the importance of these kind of observations was the Geneva Copenhagen Survey (Nordström et al. 2004) which, among other discoveries, allowed the study of chemical distributions, velocity distributions and ages in the solar neighbourhood. The development of the multi-object spectroscopy allowed to extend observations, outside the solar neighbourhood, to study large sample of stars in the different components of the Milky Way. The scientific community taking advantage of the technical developments in instruments gave rise to different Galactic surveys. In this chapter we will do a short review about few of the most important spectroscopic surveys in the past, present and future in astronomy.

\subsection{Geneva-Copenhagen survey}

The Geneva-Copenhagen survey (Nordström et al. 2004) was the first homogeneous spectroscopic survey which observed more than 1000 stars with the Danish 1.5-m telescope at ESO, La Silla, Chile, and with the Swiss 1-m telescope at Observatoire de Haute-Provence, France. It observed around 13000 stars in the solar neighbourhood, i.e. in a few hundreds of parsecs. The observed radial velocities combined with ubvy $\beta$ photometry (which allows measurements of spectral parameters as metallicity or effective temperature), parallaxes from Hipparcos and proper motions from Tycho-2, are part of the catalog of about $14000 \mathrm{~F}$ and $\mathrm{G}$ dwarfs in the solar neighbourhood (few hundreds of parsecs). 


\subsection{SDSS-II/III}

The Sloan Digital Sky Survey (SDSS; York et al. 2000) multi-fiber imaging and spectroscopic survey uses the 2.5 meters wide-angle telescope at Apache Point Observatory in New Mexico (Gunn et al. 2006) supported by the Alfred P. Sloan Foundation. The last SDSS data release (DR10) (Ahn et al. 2014) covers 14555 square degrees and it has 736484 Optical stellar spectra and 57454 Infrared stellar spectra. Figure 4.1 show the sky coverage of the full SDSS survey imaging which uses the Sloan photometric system (u,g,r,i and z). The whole SDSS program consists of several surveys but we will just refer to two (SEGUE and APOGEE) of the most important surveys for our present and future work.

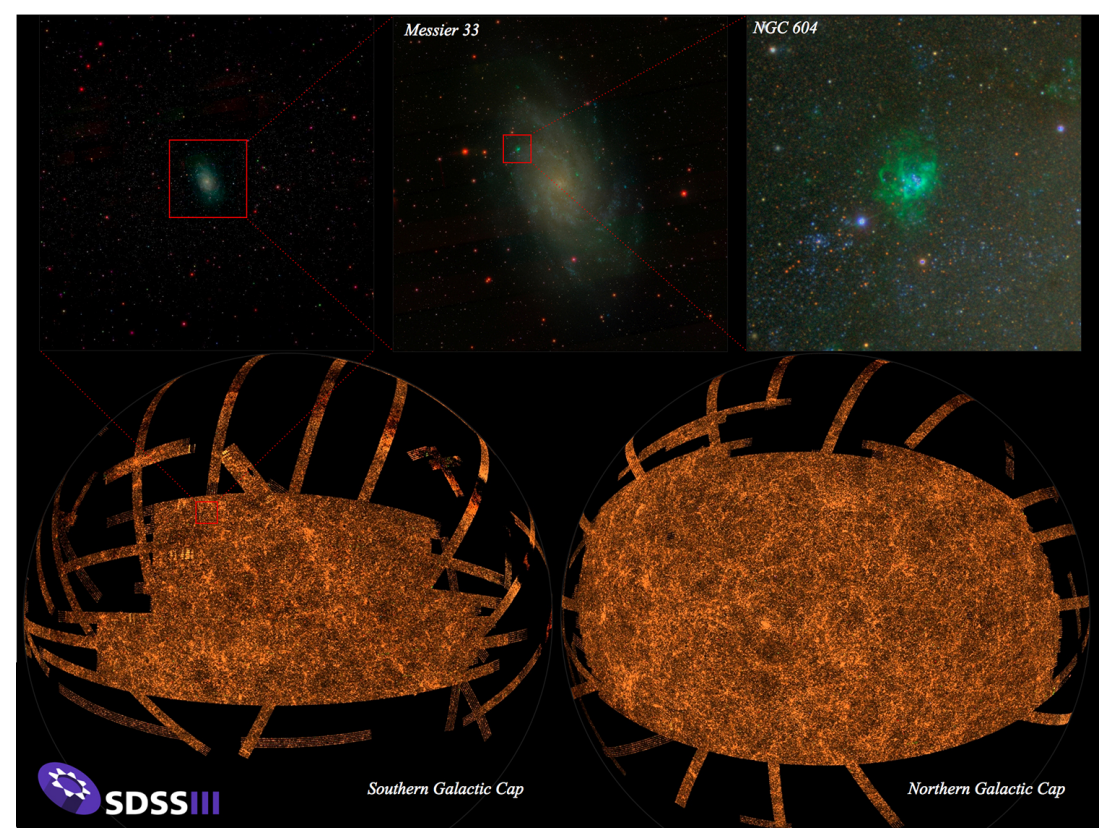

Figure 4.1: The SDSS sky coverage in the southern and northern Galactic cap. Image credit: http://www.sdss.org/sdss-surveys/

\subsubsection{SEGUE/SEGUE2}

The Sloan Extension for Galactic Understanding and Exploration (SEGUE) (Yanny et al. (2009); Eisenstein et al. (2011)) is a part of SDSS-II which obtained ugriz imaging data at moderate to low Galactic latitudes ( $|\mathrm{b}|<35$ degrees) and spectroscopy of selected stellar targets over the wavelength range $385 \mathrm{~nm}$ to $920 \mathrm{~nm}$ in order to investigate Milky Way structure. It covered around 3500 square degrees of imaging data and produced 240000 medium resolution ( $\mathrm{R} \sim 2000)$ spectra of stars with a typical signal to noise ratio of 25 to magnitude $\mathrm{g}=19$ in 1438 square degrees. In our analysis we have used this survey and specially we used the low latitude fields of this program. After the success of the survey a follow up survey (SEGUE-2) has been conceived to specially observe the halo component at high latitude fields. It observed around 119000 stars to magnitude $\mathrm{g}=19$ in 1317 square degrees. The combination of the two surveys 
creates one of the largest spectroscopic samples which combines kinematic and chemical information essential to understand the history of the Milky Way. Figure 4.2 show the spatial coverage of the spectroscopic sample for both surveys.

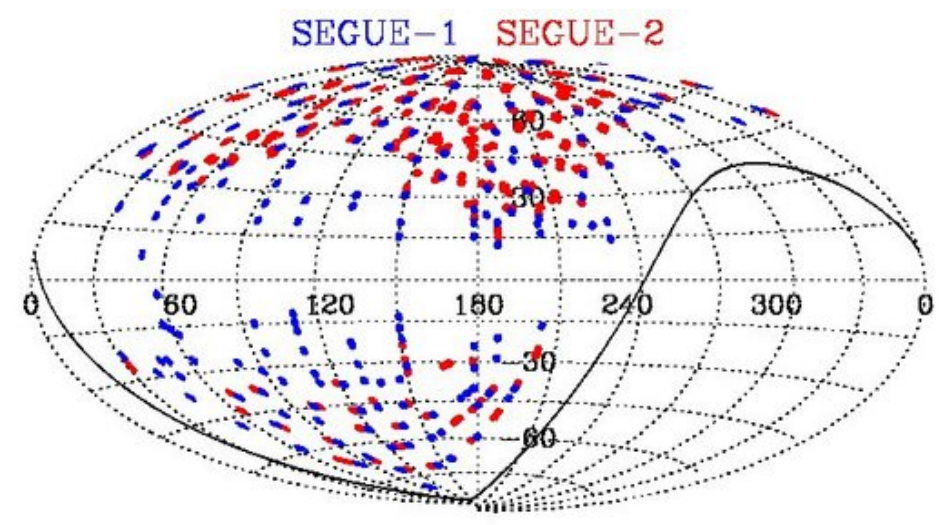

Figure 4.2: The SEGUE in blue and SEGUE-2 in red sky coverage in Galactic coordinates. Image credit: M. Strauss in http://www.sdss3.org/surveys/segue2.php

\subsubsection{APOGEE}

The APO Galactic Evolution Experiment (APOGEE; Majewski et al. 2010) uses high-resolution ( $\mathrm{R} 22$ 500), high signal-to-noise ( $\mathrm{S} / \mathrm{N}>100)$ infrared spectroscopy which allows to study in detail the halo, disc and the most inner regions of the Milky Way, like the bulge, due to the lower dependence in extinction at infrared wavelengths. The survey observes around 100000 giant stars with limiting magnitude which depends on the fields (there are fields going to $\mathrm{H}=13.8$ and fields (like the bulge) which go to $\mathrm{H}=11$ ). The survey measures spectroscopic parameters as the metallicity, effective temperature, surface gravity, and abundance of 15 different chemical species with very high precision. Figure 4.3 show the spatial coverage of the APOGEE DR10 release in Galactic coordinates and, in contrast to SEGUE, the observations are mainly located in the Galactic plane which complements SEGUE observations.

\subsection{RAVE}

The RAVE survey (the RAdial Velocity Experiment) (e.g. Steinmetz et al. (2006), Zwitter et al. (2008), Siebert et al. (2011)) was a 10 years survey (2003-2013) which gather 574630 spectra for 483330 stars. RAVE used a multi-fiber spectroscopic facility at the $1.2 \mathrm{~m}$ UK Schmidt telescope of the Anglo-Australian Observatory in Siding Spring, Australia and it is magnitude limited in the range $9<I<13$. For dwarf stars the survey is limited to distances between 50 and $250 \mathrm{pc}$ but for giants it can reach to $3.0 \mathrm{kpc}$. The survey covered 20,000 square degrees of the sky in the southern hemisphere. Figure 4.4 shows the sky coverage of the survey along with the stellar heliocentric radial velocities. The catalog has accurate radial velocities $(\sim 2 \mathrm{~km}$ 


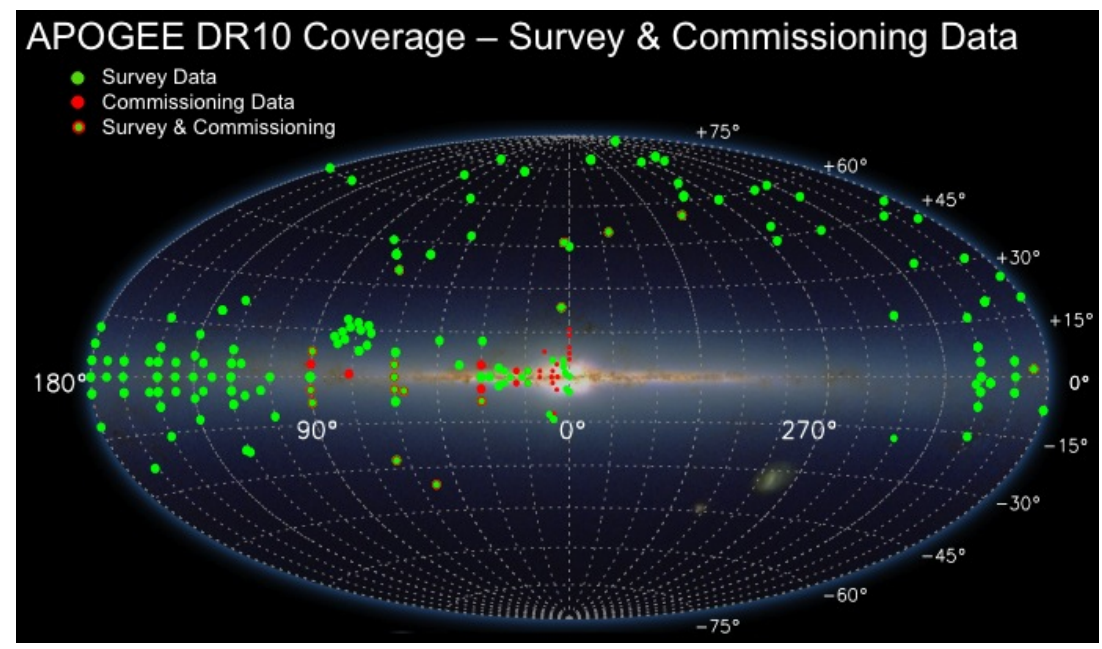

Figure 4.3: The APOGEE sky coverage, from DR10, in Galactic coordinates. Image credit: in http://www.sdss3.org/dr10/

$\mathrm{s}^{-1}$ ), stellar parameters as metallicity, effective temperature, surface gravity, elemental abundance and photometric parallaxes. The last data release (DR4) is described in Kordopatis et al. (2013a).

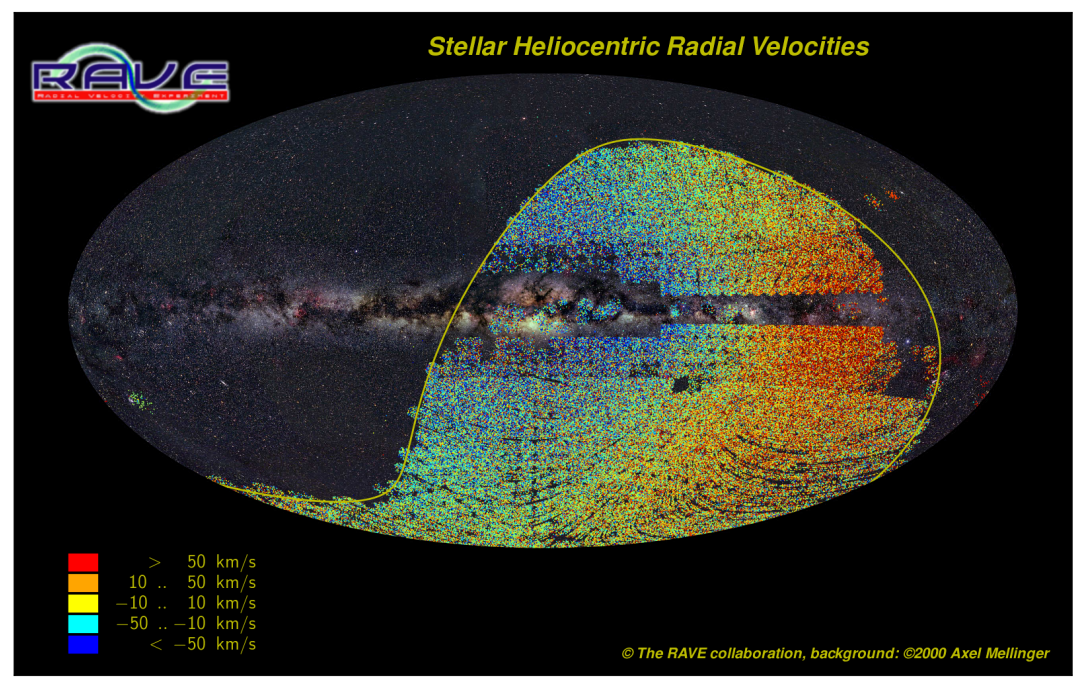

Figure 4.4: The RAVE sky coverage where color are the stellar heliocentric radial velocities. Image credit: Axel Mellinger at http://www.rave-survey.aip.de/rave/pages/project/index.jsp 


\subsection{LAMOST}

The Large Sky Area Multi-Object Fiber Spectroscopic Telescope (LAMOST: Cui et al. (2012), Zhao et al. (2012)) located in Xinglong Station of national Astronomical Observatory, China and operated by National Astronomical Observatories, Chinese Academy of Sciences (NAOC) uses a $4 \mathrm{~m}$ Xinglong Schmidt telescope with limiting magnitude of $g<20$. The LAMOST experiment for Galactic Understanding and Exploration (LEGUE: Deng et al. (2012)), has similar objectives as SEGUE, like study structure in the disc and Galactic halo or compute the metallicity distribution function in the different components. The advantage is its large aperture with a wide field of view which allows a large focal surface to accommodate up to 4000 fibers. Nevertheless, its lower resolution $(R=500$ up to $R=1800)$ and the lower $\mathrm{S} / \mathrm{N}$ produced by the instrument (due to the poor atmospheric conditions and light pollution) produce lower quality data compared with SEGUE.

\subsection{Gaia}

Gaia (Perryman et al. 2001) is an ESA mission launched in the end of 2013 and will have a five year duration. The main goal of the mission is to do the largest, more complete and more precise three-dimensional map of the Milky Way by obtaining astrometric distances from parallaxes and spectroscopic data for $V<16$, which combined with spectroscopic surveys such as the Gaia-ESO survey among others, will allow to study in great detail the structure and history of the Milky Way. It will observe more than one billion stars about $1 \%$ of the total Galactic population. In comparison the previous astrometric mission (Hipparcos Space Astrometry Mission) observed just 118218 stars (Perryman et al. 1997) i.e. around $0.012 \%$ of the final Gaia catalog. Figure 4.5 show the Galactic plane coverage of Gaia. The mission will observe the whole sky as the Two Micron All Sky Survey (2MASS; Skrutskie et al. 2006), but contrarily to the spectroscopic surveys.

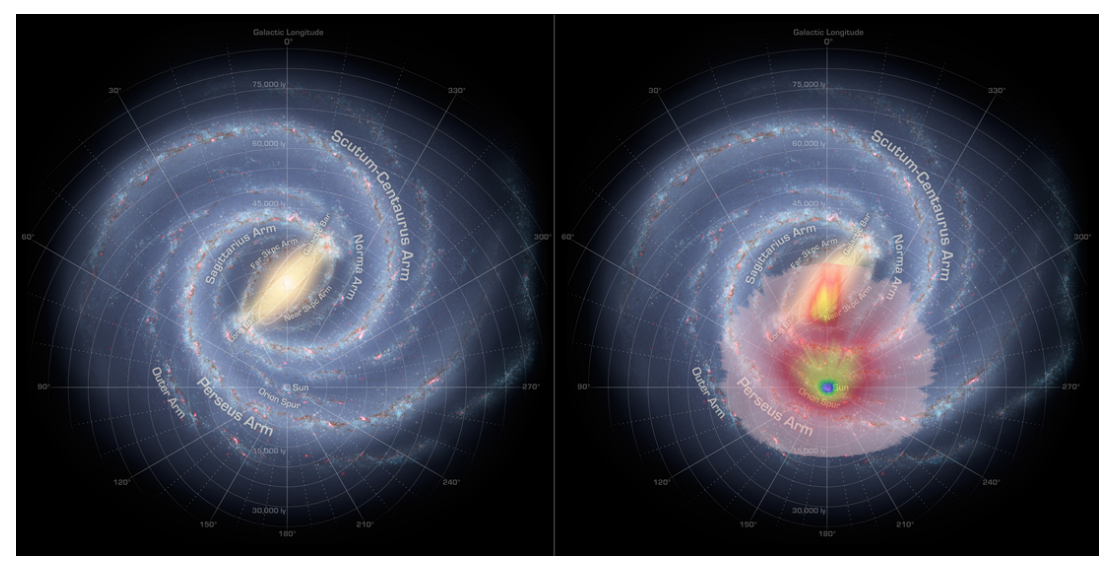

Figure 4.5: Simulation of the Gaia Galactic coverage. Image credit: X. Luri \& the DPAC-CU2 


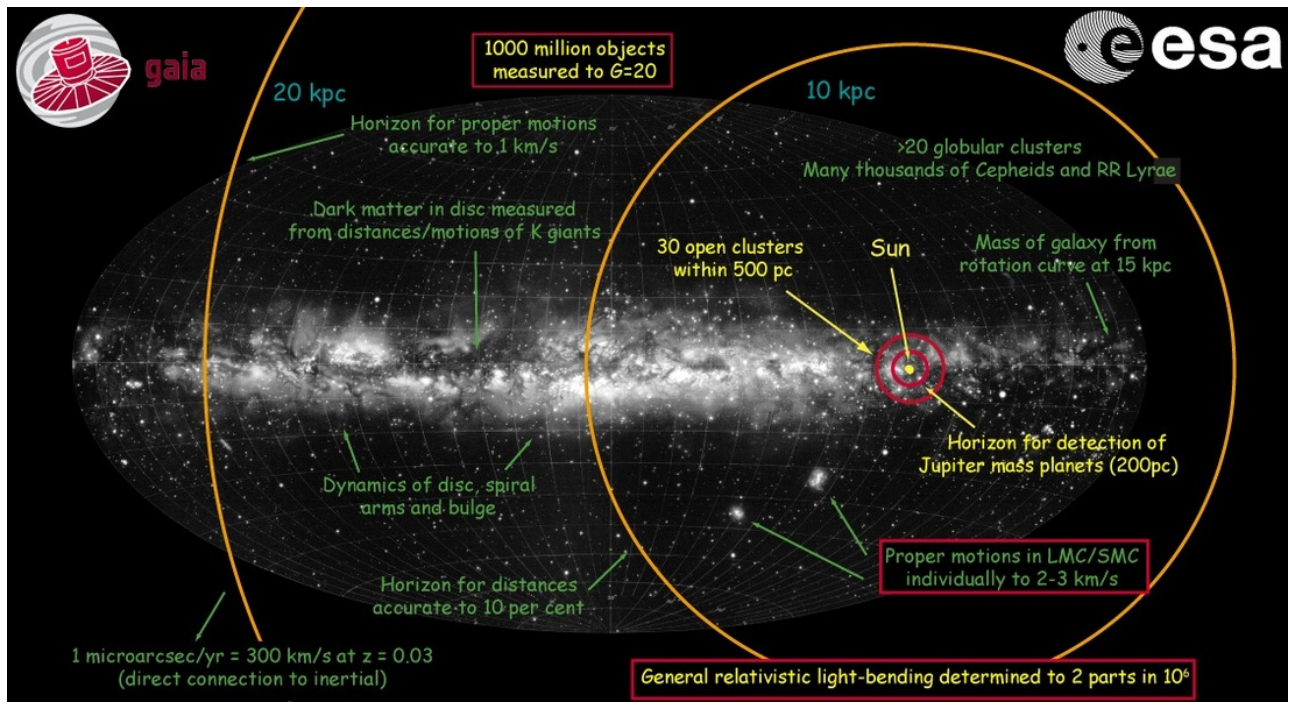

Figure 4.6: The Gaia Galactic coverage where the smaller and larger circles indicate the radius at which distances are accurate to $10 \%$ and the tangential velocities accurate to $1 \mathrm{~km} \mathrm{~s}^{-1}$. Image credit: ESA/Lund

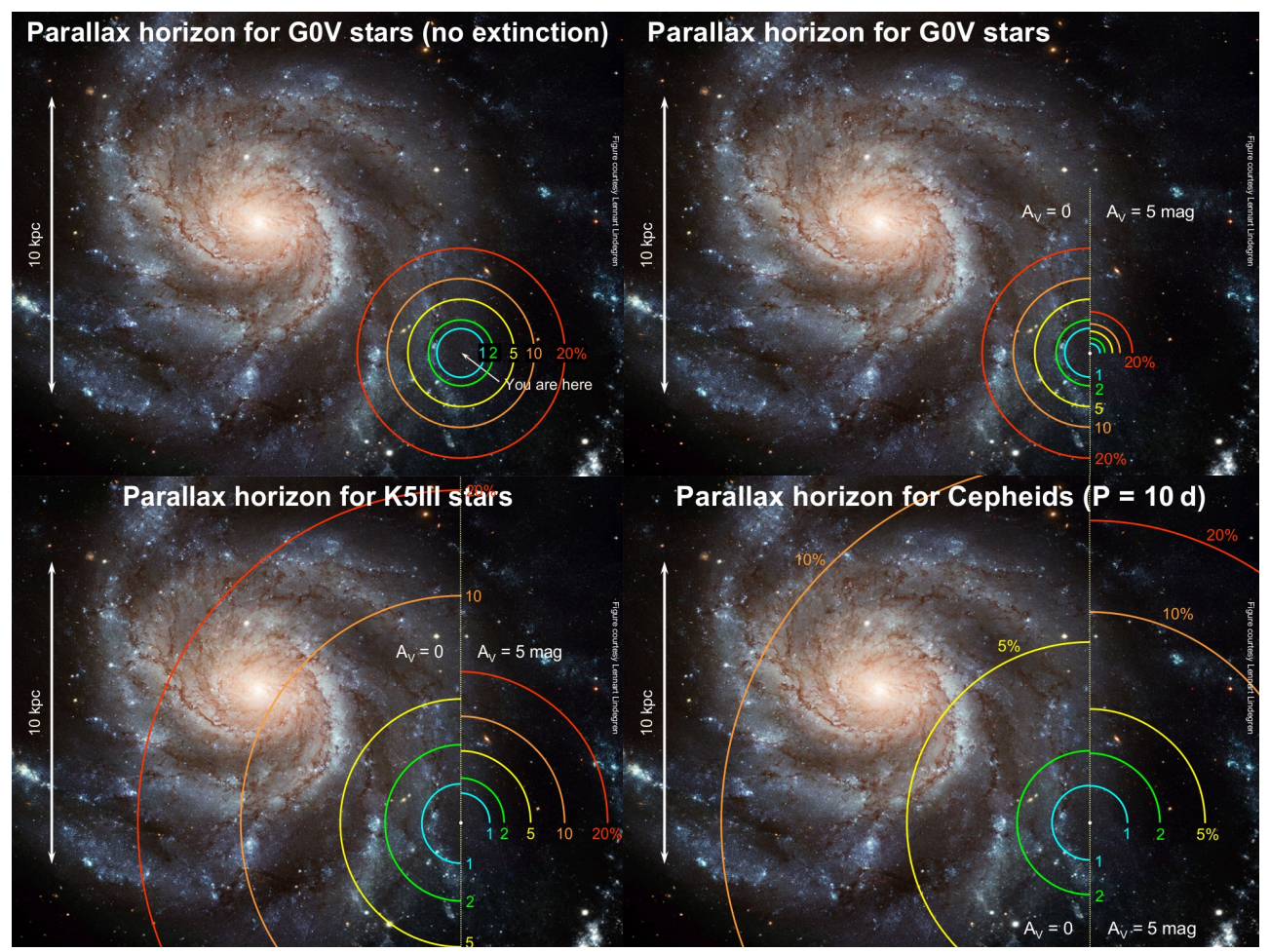

Figure 4.7: The Gaia parallax errors. Top left panel: The G0V stars parallax error distribution without extinction. Top right panel: The G0V stars parallax error distribution with extinction. Bottom left panel: The K5III stars parallax error distribution with extinction and no extinction.

Bottom right panel: The Cepheids stars parallax error distribution with extinction and no extinction Image credit: Lennart Lindegren 
The mosaic of CCDs (the largest ever in space) has a large area dedicated to astrometric measurements and smaller areas dedicated to detection, photometry and radial velocity. The Spectroscopic instrument RVS (Radial Velocity Spectrometer) will provide radial velocities with a precision estimated before the launch, of $2-10 \mathrm{~km} \mathrm{~s}^{-1}$ (for stars with $\mathrm{V} \leq 15 \mathrm{mag}$ ). Positions and parallaxes will have an estimated precision of $10 \mu \operatorname{arcsec}$ for bright stars (V 13), $300 \mu \operatorname{arcsec}$ for a G2V spectral type star at V 20 mag and proper motions a precision of 20 $\mu \operatorname{arcsec} \mathrm{yr}^{-1}$. Figure 4.6 shows the Galactic coverage along with errors in distance and proper motions. There is a first circle at $10 \mathrm{kpc}$ where it will have distances accurates to $10 \%$ and a second circle at $20 \mathrm{kpc}$ with tangential velocities better than $1 \mathrm{~km} \mathrm{~s}^{-1}$.

The estimated errors in distance will depend on the source. Figure 4.7 shows the error estimate spatial distribution for different spectral types. G dwarfs will get accurate distances for distances as far as $4 \mathrm{kpc}$ and $\mathrm{K}$ giants and Cepheids distances as accurate as $20 \%$ and $10 \%$ respectively for stars in the opposite side of the Milky Way. The figure shows that there is a large difference when the extinction is present which means that stars in the Galactic plane will have distances less precise that stars at larger distances from the plane.

The photometric precision of both photometers will be $\sim 5$ mmag for the brighter objects $(\mathrm{V} \sim 15)$ and $\sim 50 \mathrm{mmag}$ for fainter stars $(\mathrm{V} \sim 20)$. This high precision will produce low resolution spectrum which allows the derivation of spectral parameters such as the metallicity, effective temperature and surface gravity. It will produce a multi dimensional high precision catalog specially for bright stars. Ground based spectroscopic surveys, such as Gaia-ESO survey and 4-MOST, have selected sub-samples in many directions which will complement Gaia for fainter stars.

\subsection{Gaia-ESO}

The Gaia-ESO survey (GES: Gilmore et al. (2012)) is a common effort of different groups in Europe in order to produce a large sample of high resolution data, selected using VISTA (Visible and Infrared Survey Telescope for Astronomy) photometry, to combine later with the Gaia catalog. The combination of both catalogs will produce a large sample of astrometric, photometric and spectroscopic quality data. It uses FLAMES (Fibre Large Array Multi Element Spectrograph) mounted at the UT2 telescope at VLT, Chile. FLAMES is a multi-object spectrograph with 25 arcmin in diameter which feeds two different spectrograph: The high resolution spectrograph UVES (R 47000) in the wavelength range $370-950 \mathrm{~nm}$ and the intermediate resolution spectrograph GIRAFFE (R 25000 or R 10000) with central wavelength of 520, 580 and $860 \mathrm{~nm}$ depending on the setup. It dedicates, per field, up to 8 fibers to UVES and up to 130 fibers to GIRAFFE. The GES survey proposed to observe more than $10^{5}$ stars in a 5 year period covering all the populations of the Galaxy plus clusters which will allow a homogeneous analysis of the chemistry and kinematics of all components. Figure 4.8 shows the map of observed targets in the data release 1 (DR1) (Recio-Blanco et al. 2014) for the different observed target groups. The first observation was made in 31 December 2011 and in total GES will observe the sky during 300 nights divided in 9 periods plus 3 in the fourth year for compensation time and 


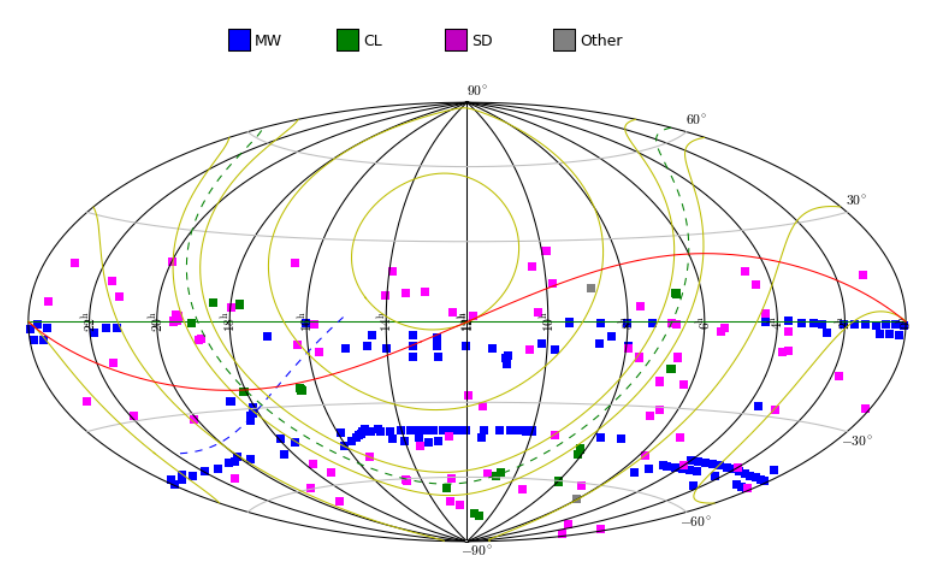

Figure 4.8: Sky coverage of the observed targets in DR1 data release. Color indicates the different target groups. MW: Milky Way fields;CL: Clusters; SD: Standard Image credit: provided by Cambridge Astronomy Survey Unit (CASU) http://casu.ast.cam.ac.uk/gaiaeso/overview

review to assess a fifth year (Gilmore et al. 2012).

\subsection{GALAH}

The GALactic Archaeology with HERMES (GALAH: Zucker et al. 2012) is a stellar survey to observe about one million stars wich uses the HERMES instrument with the Anglo-Australian Telescope of the Australian Astronomical Observatory. The survey has a limiting magnitude of $\mathrm{V}=14 \mathrm{mag}$ and will observe half of the accessible sky for latitudes larger than $|\mathrm{b}|>10^{\circ}$ in high resolution ( $\mathrm{R} \sim 28000$ and $\mathrm{R} \sim 45000$ ). HERMES has 390 fibers distributed in $\pi$ square degrees and covers wavelengths in the range 4718 to $7890 \AA$. This set allows HERMES to obtain precise chemical abundances for 15 different elements and radial velocities. The main targets of the selection sample have a fractional contribution, as given by the GALAXIA code (Sharma et al. 2011), for the thin (0.58 dwarfs and 0.19 giants), thick (0.11 dwarfs and 0.07 giants) disc and halo (0.02 dwarfs and 0.03 giants) and are selected using the 2MASS survey (Table 2: Zucker et al. 2012). Observations (around 63,892 stars over 180 fields until August 2014) for the pilot survey and GALAH Survey are being made and the data is being processed by the survey team. The First Data Release is expected in January 2015. 


\subsection{LSST}

The Large Synoptic Survey Telescope system (LSST: LSST Science Collaboration et al. 2009) is a very deep (from $r<24.5$ in a single observation to $r<27.5$ in the final release) wide-field survey of the southern sky which will use a $8.4 \mathrm{~m}$ primary mirror telescope in the proposed site, Cerro Pachón in Northern Chile. In ten years it will observe each path of the sky about 1000 times. It will obtain quality imaging (using SDSS photometric system) and astrometry for a large field of view of 9.6 square degrees (diameter is equal to 3.5 degrees) made by the world largest digital camera with 3200 Megapixels. To reach high precision astrometry it has to maintain the limit set by the atmosphere at 10 mas per observation which will allow to obtain a precision in parallaxes around 1 mas and a proper motion uncertainty of 0.2 mas $\mathrm{yr}^{-1}$. In this sense it is going to complement the Gaia survey with similar accuracies but at much fainter magnitude.

\subsection{WEAVE}

The WEAVE (A new wide-field multi-object spectrograph for the William Herschel Telescope) survey (Dalton et al. 2012) is planned for the 4.2-m William Herschel Telescope at the Observatorio del Roque de los Muchachos, on La Palma in the Canary Islands. Each exposure can observe up to 1000 objects which feed a single spectrograph with resolutions of $\mathrm{R} \sim 5000$ for stars fainter than $\mathrm{V}=17.0$ mag and $\mathrm{R} \sim 20000$ for stars brighter than $\mathrm{V}=18$ mag (Dalton et al. 2012). The low-resolution mode will observe more than $\sim 10^{6}$ stars with a radial velocity precision better than $5 \mathrm{~km} \mathrm{~s}^{-1}$ in the range $17.0<\mathrm{V}<20.0$. The high-resolution mode will provide chemical abundances for most of the elements with an accuracy of $\sim 0.1$ dex. WEAVE will be complemented by the 4-MOST survey which will cover the southern sky which is inaccessible to WEAVE. Science operations are schedule to start around 2017.

\subsection{MOST}

The 4-metre Multi-Object Spectroscopic Telescope (4MOST: de Jong et al. 2012) is a survey using the Visible and Infrared Survey Telescope for Astronomy (VISTA) 4.1 metre telescope, located at the Paranal Observatory in Chile. The instrument has a large field-of-view and will cover the southern sky by obtaining spectra of around 2400 objects distributed over an area of 4 square degrees. The survey will probably begin to observe in 2019 and in the final 5 years period it should deliver around 30 million spectra covering from 15000 to 20000 square degrees. It will measure abundances up to 15 elements for stars brighter that $16 \mathrm{~V}$-mag. The low resolution spectrograph goals are a resolution larger than 5000 over the full wavelength range $(390-1050 \mathrm{~nm})$ and larger than 7000 at $800 \mathrm{~nm}$. The high resolution goals are a resolution larger than 18000 for a range of wavelength between $390 \mathrm{~nm}$ and $456.5 \mathrm{~nm}$ and larger than 20000 in average for a wavelength range of 585-677 nm (de Jong et al. 2012). Its high resolution makes 
it the natural sucessor of the Gaia-ESO survey and the ideal complement of Gaia survey which overlaps it in magnitude limits.

\subsection{MOONS}

The Multi-Object Optical and Near-infrared Spectrograph (MOONS: Cirasuolo et al. 2012) consists in $\sim 1000$ fibers distributed in a large field of around 500 square arcminutes which will use the $8.2 \mathrm{~m}$ Very Large Telescope (VLT). The instrument has a medium resolution mode ( $\mathrm{R} \sim 4000-6000$ ) wich covers the wavelengths from $0.8 \mu \mathrm{m}$ to $1.8 \mu \mathrm{m}$ and a high resolution mode which allows to measure radial velocities ( $\mathrm{R} \sim 8000$ around the CaII triplet) and detailed measurements of chemical abundances $(\mathrm{R} \sim 20000)$ for more that 2 million stars ranging from $\sim 14.5$ mag to $\sim 20.5 \mathrm{mag}$ in $\mathrm{V}$ band. These requirements make this survey a complement to APOGEE survey by covering fainter magnitudes in the near-infrared. The instrument will be operational by 2019 . 


\section{Chapter 5}

\section{Photometric and spectroscopic sample}

The objective of our work is to understand the structure formation and evolution of the Milky Way. The model can give constraints on the formation and structure by comparing simultions with real data. For this study we use the SEGUE (Yanny et al. (2009); Eisenstein et al. (2011)) sample, part of the Sloan Dogital Sky Survey (York et al. 2000). We use 10 low latitude fields which have bright $(\mathrm{g}=15-18 \mathrm{mag})$ and faint plates $(\mathrm{g}=17.5-19.5 \mathrm{mag})$ presented in table 5.1. We have two fields in the inner disc direction, five at intermediate longitudes and three at the anticenter direction. These fields cover a small range of galactic latitudes from $|8.0|$ to | 16.0 | but are located at directions where we can have a significant thick disc contribution. The photometric data will provide constrains on the shape and structure of the Milky Way while the spectroscopic sample contributes to study the formation and evolution of the Milky Way by constraining the metallicity distribution and how this distribution changed with time. We have used two different processes to select the photometric sample and the spectroscopic sample. In the next section we will describe how we have selected the photometric sample. Section 5.2 describes the method used to select the spectrocopic sample.

\subsection{Selecting the photometric sample from SEGUE}

We retrieved data using the SDSS database accessible on line at casjobs ${ }^{1}$. For each field we selected targets from the Sloan Digital Sky Survey Data Release 9 (DR9; Ahn et al. 2012) in a radius of 90 arcmin of the plate center. We use the function fGetNearbyObjEq() to get objects. If we do not want to have all objects but just stars we have to specify using plmatch.type $=6$. We can match up and retrieve the photometric information for each star from PhotoObjAll. We also remove all duplicate observations checking a variable called "mode", or equivalently doing (resolve_status \& survey_PRIMARY) !=0. The sample is cleaned from regions around bright sources, because nearby stars can have unreliable photometry, and we checked for quality flags. After a study about the flags we decided to exclude four flags in two different bands, $\mathrm{g}$ and $\mathrm{i}$

\footnotetext{
${ }^{1}$ http://casjobs.sdss.org/CasJobs/
} 
Table 5.1:

SEGUE survey plates used for the present analysis

\begin{tabular}{lcccc}
\hline \hline Plate bright/faint & $\begin{array}{c}1 \\
\left(^{\circ}\right)\end{array}$ & $\begin{array}{c}\mathrm{b} \\
\left(^{\circ}\right)\end{array}$ & $\begin{array}{c}\mathrm{Ra} \\
\left(^{\circ}\right)\end{array}$ & $\begin{array}{c}\text { Dec } \\
\left(^{\circ}\right)\end{array}$ \\
\hline $2534 / 2542$ & 50 & 14 & 277.60 & 21.33 \\
$2536 / 2544$ & 70 & 14 & 286.66 & 39.11 \\
$2537 / 2545$ & 110 & 10.5 & 334.17 & 69.39 \\
$2538 / 2546$ & 110 & 16 & 323.07 & 73.64 \\
$2554 / 2564$ & 94 & 14 & 302.97 & 60.01 \\
$2555 / 2565$ & 94 & 8 & 312.39 & 56.59 \\
$2556 / 2566$ & 94 & -8 & 330.15 & 45.06 \\
$2668 / 2672$ & 187 & -12 & 79.49 & 16.61 \\
$2678 / 2696$ & 187 & 8 & 98.13 & 26.67 \\
$2681 / 2699$ & 178 & -15 & 71.50 & 21.98 \\
\hline \hline
\end{tabular}

Table 5.2:

Photometric flags used

\begin{tabular}{|c|c|}
\hline Flag & Description \\
\hline EDGE & Stars are too close to the edge of the frame \\
\hline EGHOST & Electronic ghost from a bright star \\
\hline SATURATED & Saturated pixels from a bright star \\
\hline NOTCHECKED & $\begin{array}{l}\text { Stars weren't checked for } \\
\text { peaks by deblender }\end{array}$ \\
\hline BINNED1 & $\begin{array}{l}\text { Select stars with a peak detection } \\
>5 \text { sigma in the original imaging frame }\end{array}$ \\
\hline NOPROFILE & Select stars with NOPROFILE $=0$ \\
\hline
\end{tabular}

and used two flags to select stars. First, we don't want stars that are too close to the edge of the frame to be measured, so we removed stars associated with the flag "EDGE". We also do not want objects associated with the flags "EGHOST"and "SATURATED", because it can have respectively a electronic ghost or saturated pixels from a bright star. Stars that have not been checked ("NOTCHECKED") for peaks by deblender are removed because the deblending may be unreliable.

Furthermore, we selected stars using the flag "BINNED1"to select only stars with a peak detection higher than 5 sigma in the original imaging frame. The last flag used was the "NOPROFILE"from which we select stars with not NOPROFILE (flag equal to zero) because the photometric quantities measured from it are likely to be suspect due to the invalid radial profile. Table 5.2 summarizes the photometric flags used. 
We estimated the completeness of the sample to be between 14.0 and 19.5 mags in $\mathrm{g}$ band. We then plotted colour-colour diagrams (g-r vs r-i), for the observations, and noticed that the locus was slightly enlarged compared with simulations in a few CCD frame of the mosaic. We disregarded the photometry from these CCD frames. This procedure was done for all plates and in more than half of them we found this effect. The effective area for each plate is around $85 \%-90 \%$ of the plate area.

\subsection{Selecting the spectroscopic sample from SEGUE}

The spectroscopic sample is selected using different flags than the photometric one. The Spectrocopic data used in this work are from the SEGUE survey Data Release 8 (DR8;Aihara et al. $(2011)))^{2}$. We selected stars that do not have a critical flag category (Table 5.3) by the SEGUE Stellar Parameter Pipeline (SSPP; Lee et al. (2008a,b); Allende Prieto et al. (2008)).

Table 5.3:

Spectroscopic flags used

\begin{tabular}{lc}
\hline \hline Flag & Description \\
\hline $\mathrm{D}$ & Likely white dwarf \\
$\mathrm{d}$ & Likely sdO or sdB \\
$\mathrm{H}$ & Hot star $T_{e f f}>10000 K$ \\
$\mathrm{~h}$ & Helium line detected, possibly very hot star \\
$\mathrm{E}$ & Emission lines in the spectrum \\
$\mathrm{S}$ & Sky spectrum \\
$\mathrm{V}$ & No radial velocity information \\
$\mathrm{B}$ & Too blue $g-r<-0.3$ \\
$\mathrm{R}$ & Too red $g-r<1.3$ \\
$\mathrm{X}$ & No parameters estimate \\
\hline \hline
\end{tabular}

The pipeline SSPP was made to analyze high galactic latitude observations and it uses photometry corrected using the reddening maps from Schlegel et al. (1998). Cheng et al. (2012b) showed that the $T_{\text {eff }}$ estimates from SSPP pipeline which use photometric information are systematically higher than the value obtained from the spectrum only when extinction is high. So, using a similar approach, we computed a new $T_{\text {eff }}$ from a weighted average over spectroscopic methods. The methods used to compute the new corrected effective temperature are ki13, ANNSR, ANNRR, NGS1 and HA24 (See Lee et al. (2008a) for details about the methods). For stars with temperatures lower than $5000 \mathrm{~K}$ it is more robust to use alone the NGS1 method (Private comunication from Young Sun Lee). The weights for each method are based on the error associated to each method. In figures 5.1 and 5.2 we plot the difference between

\footnotetext{
${ }^{2}$ http://casjobs.sdss.org/CasJobs/
} 
the SSPP temperature estimates and the corrected values as a function of the SSPP temperature estimates and of the extinction $\mathrm{E}(\mathrm{B}-\mathrm{V})$ respectively. We can see in figure 5.1 a discrepancy between temperatures, for $T_{\text {eff }}<4900 \mathrm{~K}$. For safety we choose to use only data with $T_{\text {eff }}>4900$ $\mathrm{K}$.

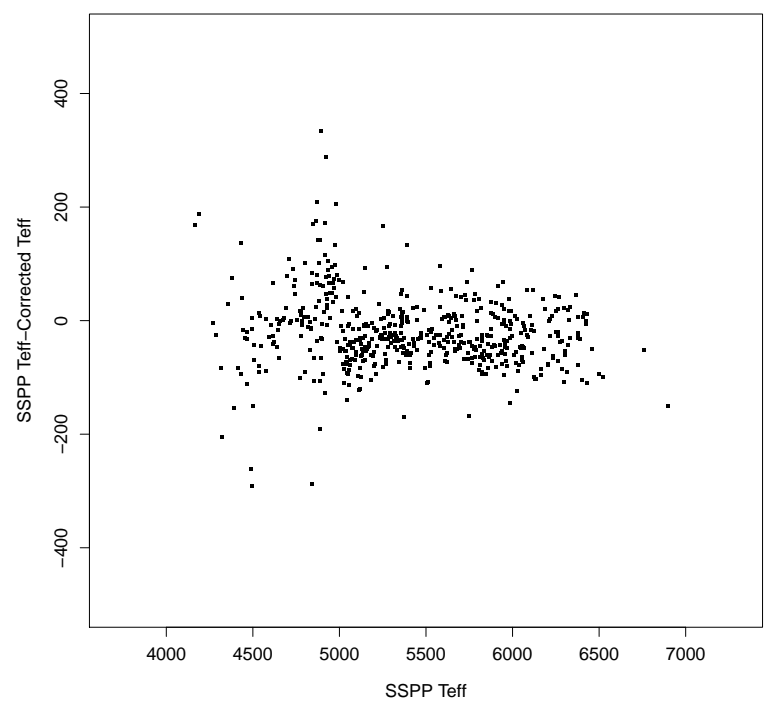

Figure 5.1: Difference between the SSPP temperature estimates and the corrected values as a function of the SSPP temperature estimates for plate $2536(1, b)=\left(70^{\circ}, 14^{\circ}\right)$.

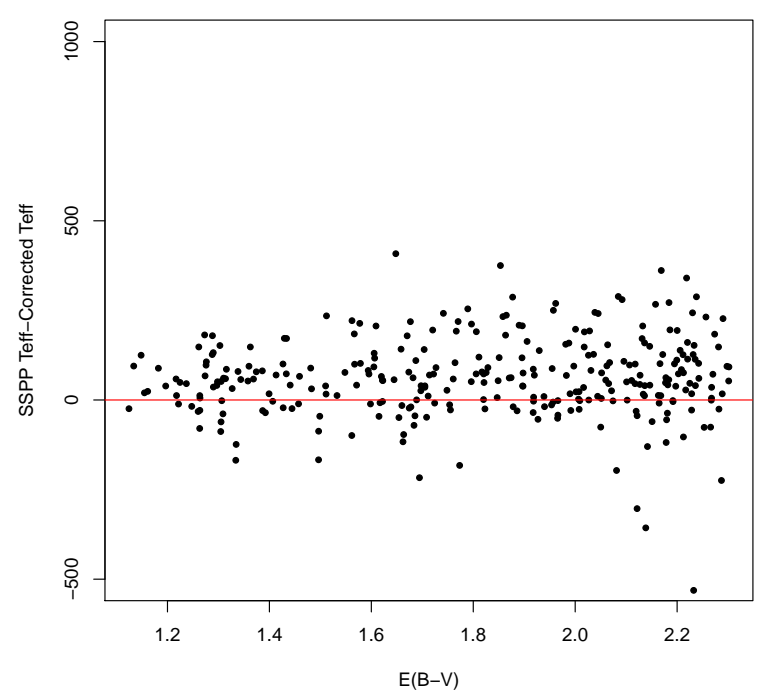

Figure 5.2: Difference between the SSPP temperature estimates and the corrected values as a function of the extinction $\mathrm{E}(\mathrm{B}-\mathrm{V})$ for plate $2555(1, \mathrm{~b})=\left(94^{\circ}, 8^{\circ}\right)$. 


\section{Chapter 6}

\section{Simulations}

\subsection{Masking the simulations}

The simulated catalogs were obtained from the Besançon galaxy model by doing a rectangular selection in galactic coordinates with center on the center of the field. We apply cuts in magnitude (g band), color (g-r) and coordinates on the simulated catalogues as has been done on the observations. The simulated stars are placed randomly in the field in contrast with the observed data (Fig 6.1).

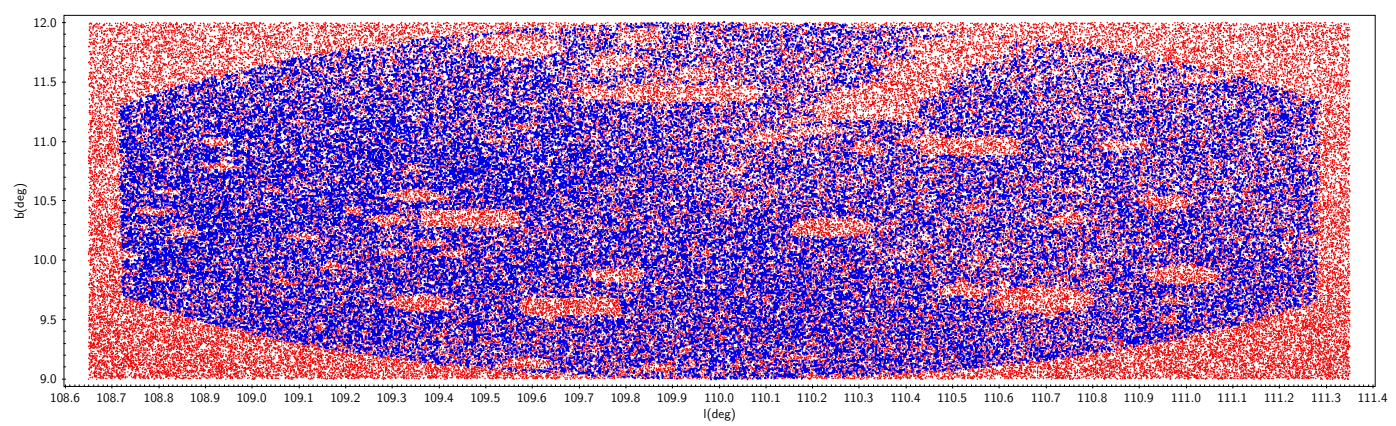

Figure 6.1: Distribution in (1,b) of the simulated stars (red points) and observed ones (blue points) for the field 2537.

A masking code has been developed and applied to the simulations to take into account the empty regions around bright stars and CCD frames discarded. The masking code is based on a simple galactic coordinate binning. We bin, with a step (between $0.02^{\circ}$ and $0.04^{\circ}$ ) that depends on the field, all stars from the data catalogue and we obtain the number density, for each of these squares. We proceed in the same way for the simulated catalogue and we reject stars that are inside squares of null density. We show in figure 6.2 the result of the masking on field 2537. We check that the processes do not eliminate stars in regions outside the holes. 


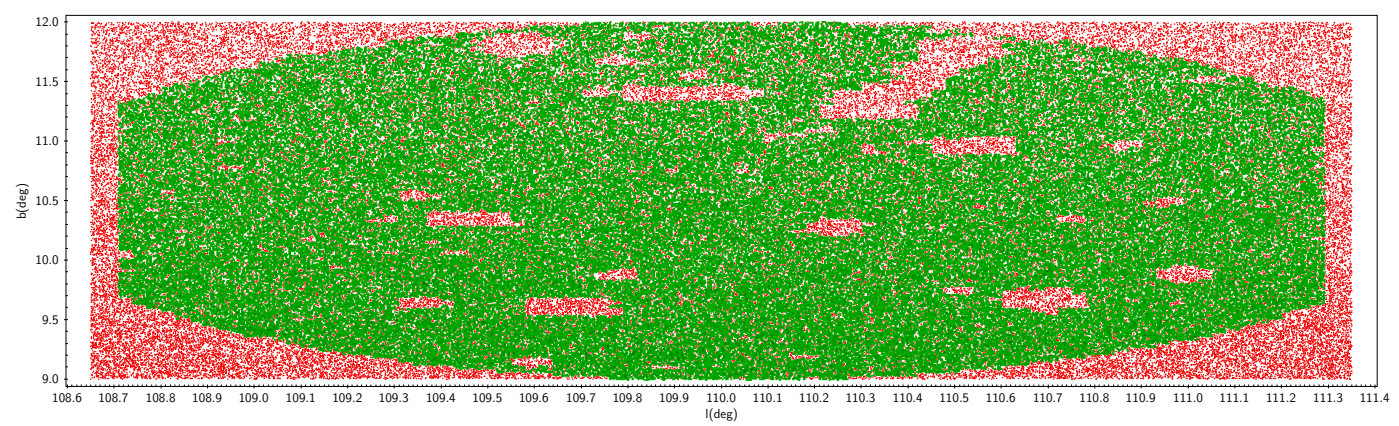

Figure 6.2: The green points are the masked simulation and the red points are the full simulation for the field 2537.

\subsection{Extinction}

From figure 6.1 we see the difference in star density between the right and left side of the observed field due to extinction in these low latitude fields. The next step was to overcome the extinction effects.

Simulated stars have to be reddened accordingly to real data. To simulate extinction, we considered 3 extinction models:

- Schlegel et al. (1998) (hereafter SFD maps) is a full-sky map based in COBE/DIRBE and IRAS/ISSA maps. Since it gives the total line of sight extinction it overestimates the extinction of stars which are at short distances.

- Drimmel \& Spergel (2001) (hereafter DS maps) a complexe model of the extinction which takes into account spiral arms, including the local arm, and is calibrated on SFD maps for the total extinction.

- Marshall et al. (2006) is a 3D extinction model based on the Besançon Galaxy model. Unfortunately the maps have been done for low latitudes only $(|b|<10)$ and cannot be used in the majority of the fields described in this work.

We inspected the extinction distribution across the different fields in the SFD maps. We noticed that the extinction is highly variable across the fields in the total extinction in $\mathrm{g}$ band $\left(\mathrm{A}_{g}\right)$. In the most favorable case the extinction range can be of 1 magnitude, but in some fields it can reach 4 to 8 magnitudes. In figures 6.3 and 6.4 we map the Schlegel et al. (1998) extinction in galactic coordinates, which allows to inspect the large extinction variation. 


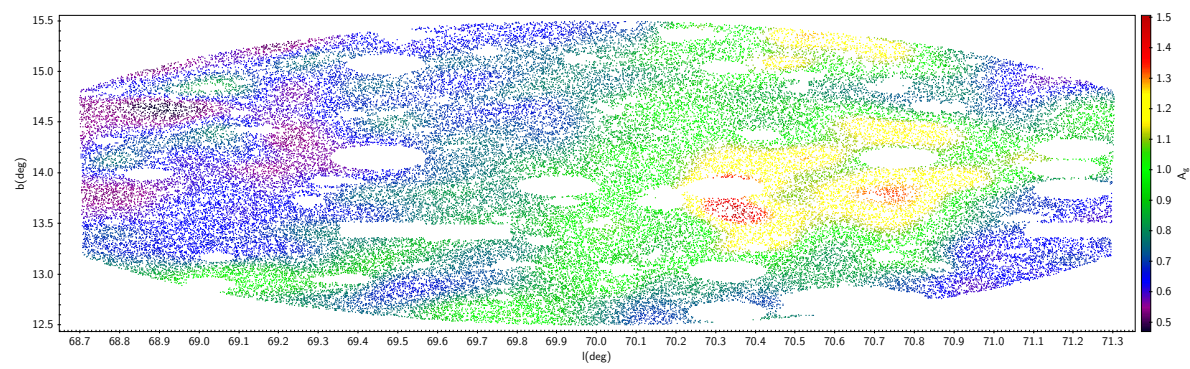

Figure 6.3: Map of the Schlegel et al. (1998) extinction in the g band for the field 2536. We can see in this figure regions where the extinction is homogeneous and low and regions where the extinction is higher.

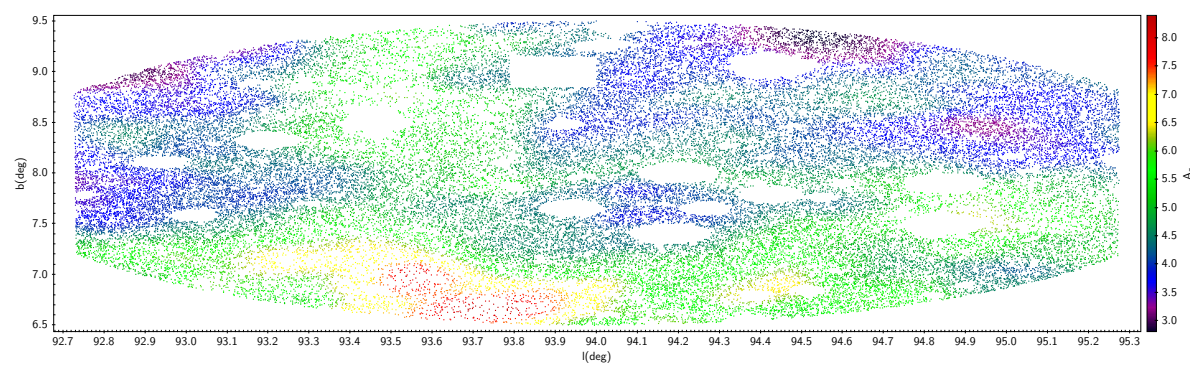

Figure 6.4: Map of the Schlegel et al. (1998) extinction in the g band for the field 2555. We can see in this figure regions where the extinction in $\mathrm{g}$ band can reach 8 magnitudes.

The most suitable extinction model for our purpose is the Drimmel \& Spergel (2001) (hereafter DS) model because is a 3D full-sky extinction model. However DS maps have lower resolution than SFD maps. In figure 6.5 we present the field 2536, in galactic coordinates where absorption is color coded. The squares mark the spatial resolution of the model. Although there is not a smooth transition, compared to Schlegel et al. (1998), between regions we still recover the regions were the extinction has a maximum in the Schlegel et al. (1998) extinction maps.

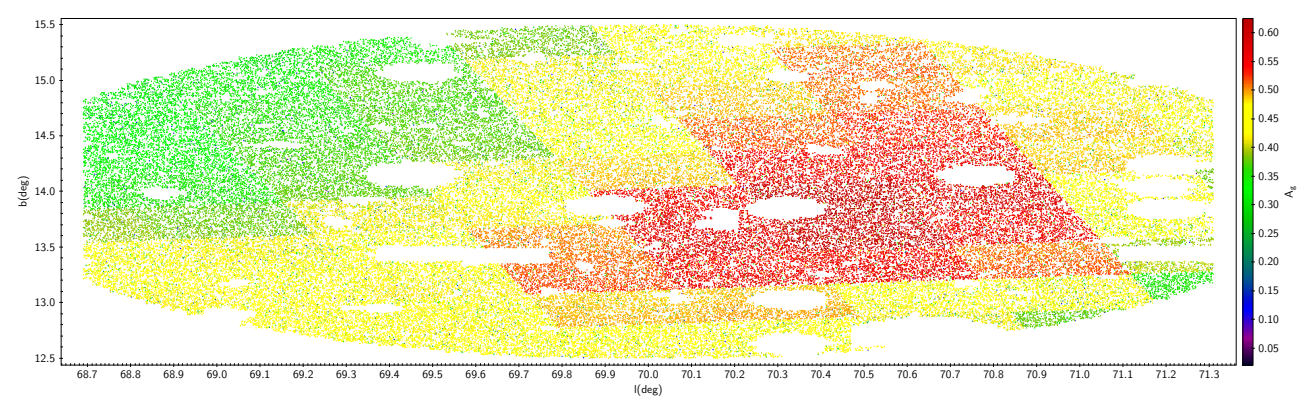

Figure 6.5: Map of the Drimmel \& Spergel (2001) extinction model in the g band for the field 2536. 


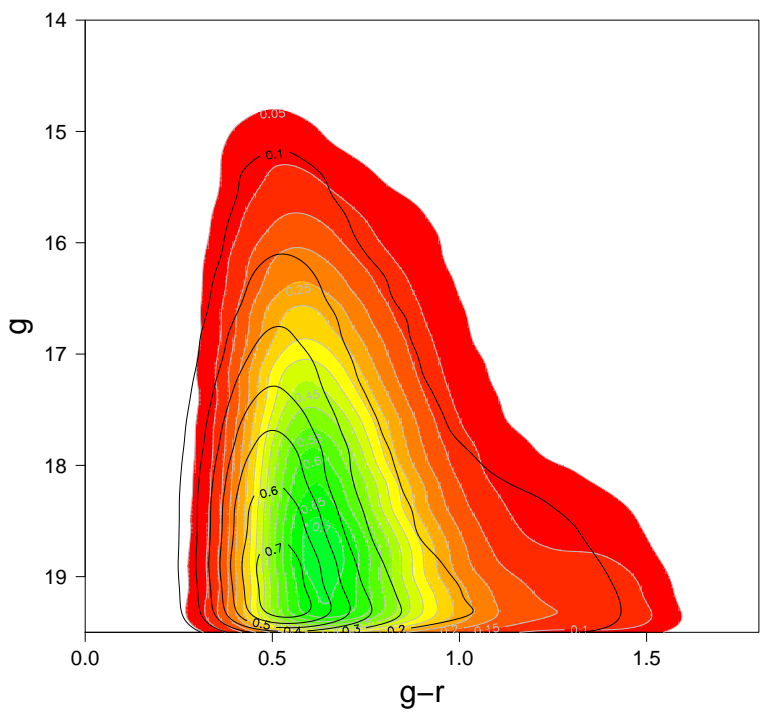

Figure 6.6: Color magnitude diagram for the field $2536(1, b)=\left(70^{\circ}, 14^{\circ}\right)$. Density map along with grey contours are observations. Black contours are simulations.

The relation between the extinction and reddening is given by $E(B-V)=A_{V} / R_{V}$ where $R_{V}=$ 3.1 is a single extinction law assuming a diffuse gas with reasonably uniform dust properties in the visible (Schultz \& Wiemer 1975; Schlegel et al. 1998). $R_{V}$ can take values in the between 2.5 and 6 for different lines of sight but in general $\sim 3.1$ is correct. The reddening is related with extinction in the Sloan photometric system by the following equations 6.2 (Table 22 from Stoughton et al. (2002)).

$$
\begin{aligned}
& A_{u}=5.155 \times E(B-V) \\
& A_{g}=3.793 \times E(B-V) \\
& A_{r}=2.751 \times E(B-V) \\
& A_{i}=2.086 \times E(B-V) \\
& A_{z}=1.479 \times E(B-V)
\end{aligned}
$$

After applying the DS maps to our simulations we plotted the CMD for the different fields. We compare the density in the simulation with SDSS data for two fields in figures 6.6 and 6.7. Figure 6.6 shows a small shift of the turnoff color of the simulated CMD by an amount of 0.15 at maximum in g-r. Figure 6.7 compares the star density in the CMD for field 2555 where the extinction is larger. The model is clearly too blue by $\sim 0.6$ mag because DS map underestimates extinction in this field.

We developed a method to correct the magnitudes and color of stars in the simulated catalogue after the application of the Drimmel \& Spergel (2001) extinction model. We estimated the color peak in $\mathrm{g}-\mathrm{r}$ and $\mathrm{r}-\mathrm{i}$ in each $\mathrm{g}$ magnitude bin and we minimized the distance between 


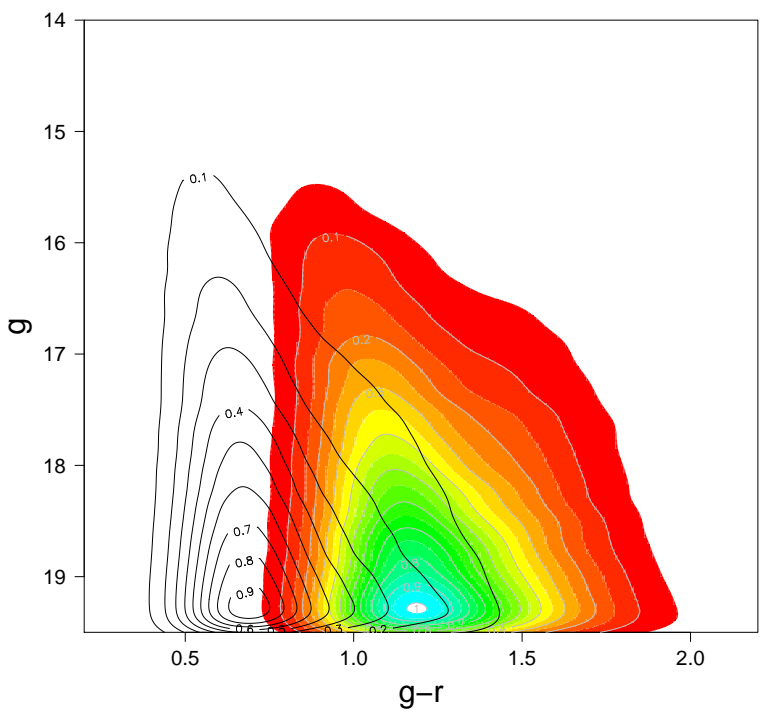

Figure 6.7: Color magnitude diagram for the field $2555(1, b)=\left(94^{\circ}, 8^{\circ}\right)$. Density map along with grey contours are observations. Black contours are simulations.

the color peaks of the simulations and observations. To correct the extinction we assumed that the correction depends on the distance by minimizing the deviation as a first order polynomial and we constraint the coefficients.

$$
y_{\text {star }}(d)=b_{0}+a_{1} d
$$

where $y_{\text {star }}(d)$ is the correction applied to each star $b_{0}, a_{1}$ are the parameters to constrain and $\mathrm{d}$ is the distance in kpc. To obtain the best solution we use a modified $\chi^{2}$ minimization. The result was not satisfactory when using the whole field, because different parts of the fields required different corrections. Considering that the majority of the low latitude fields is highly inhomogeneous, as shown above, we have divided each field in 9 areas. The total $\mathrm{A}_{v}$ as a function of the distance to the star before and after correction is shown for all fields from figure 6.8 to figure 6.17. Table 6.1 shows measures of central tendency (Mean and Mode) and of dispersion (Standard deviation) of the DS (d) and DS with correction (dc) extinction distribution for each field. The extinction dispersion is larger after the correction. The results show fields, as the field 2555, which needed a larger extinction correction and fields, as the 2556 and 2678, which the DS extinction model corrects better. We show in Table 6.2 the results for the coefficients for one simulation by field for a correction to the DS extinction model. 

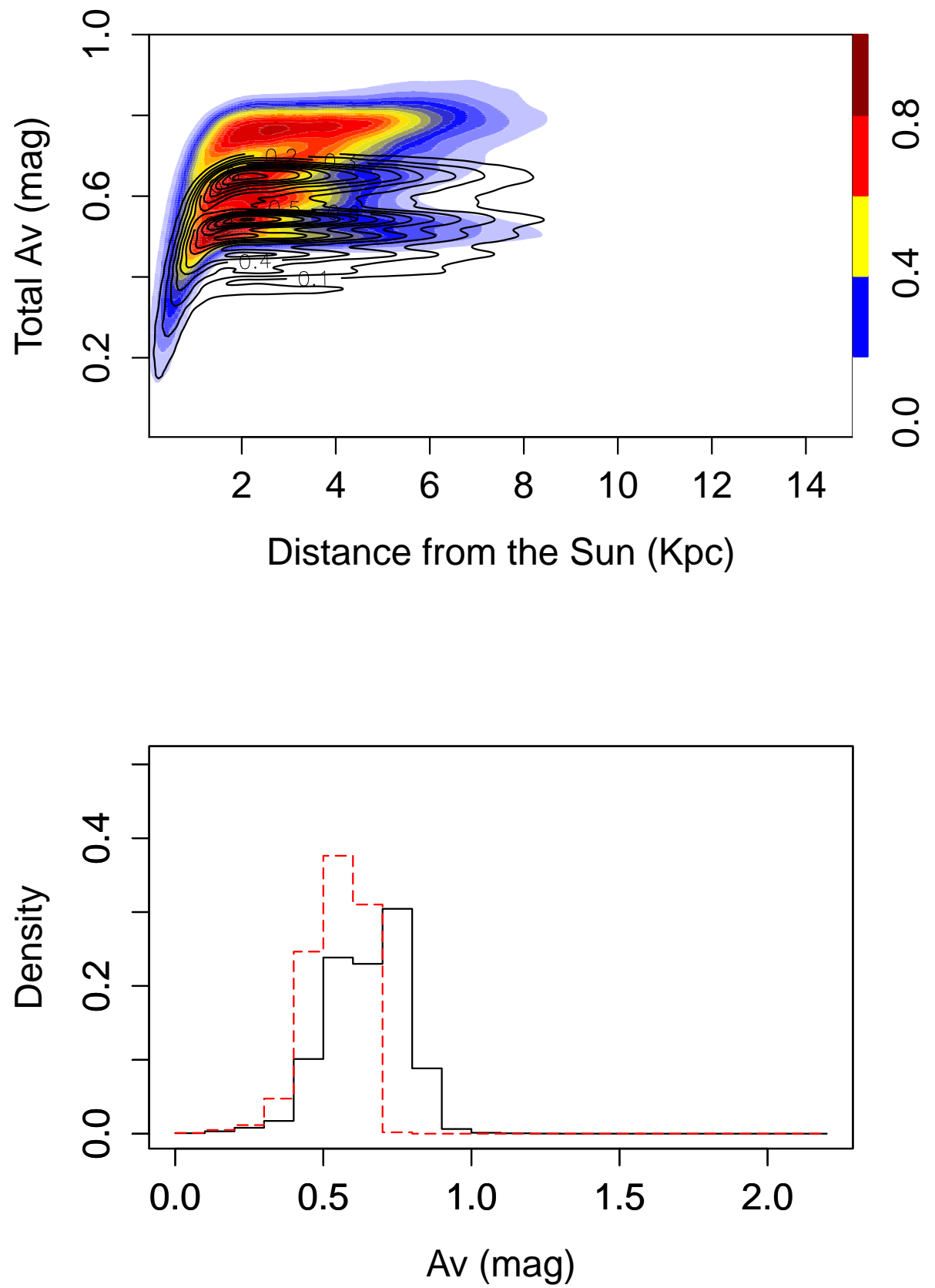

Figure 6.8: Top panel: Total $A_{v}$ as a function of the distance from the Sun for field 2534 $(1, b)=\left(50^{\circ}, 14^{\circ}\right)$. Density contours are the total $\mathrm{A}_{v}$ after correction and black contours are before the correction. Bottom panel: Total $\mathrm{A}_{v}$ distribution before (red dashed line) and after correction (solid black line). 

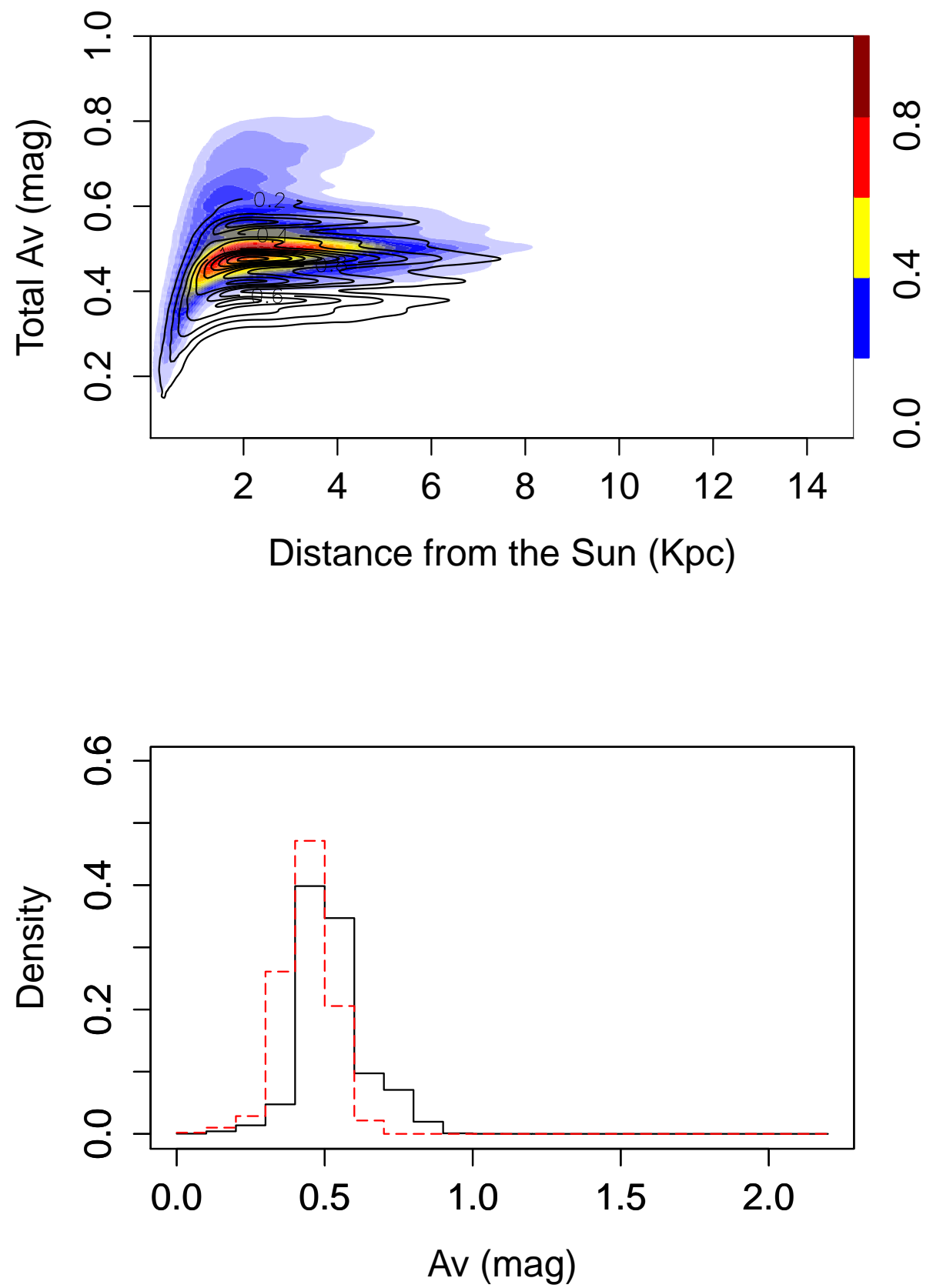

Figure 6.9: Total $A_{v}$ as a function of the distance from the Sun for field $2536(1, b)=\left(70^{\circ}, 14^{\circ}\right)$. Density contours are the total $\mathrm{A}_{v}$ after correction and black contours are before the correction. Bottom panel: Total $\mathrm{A}_{v}$ distribution before (red dashed line) and after correction (solid black line). 

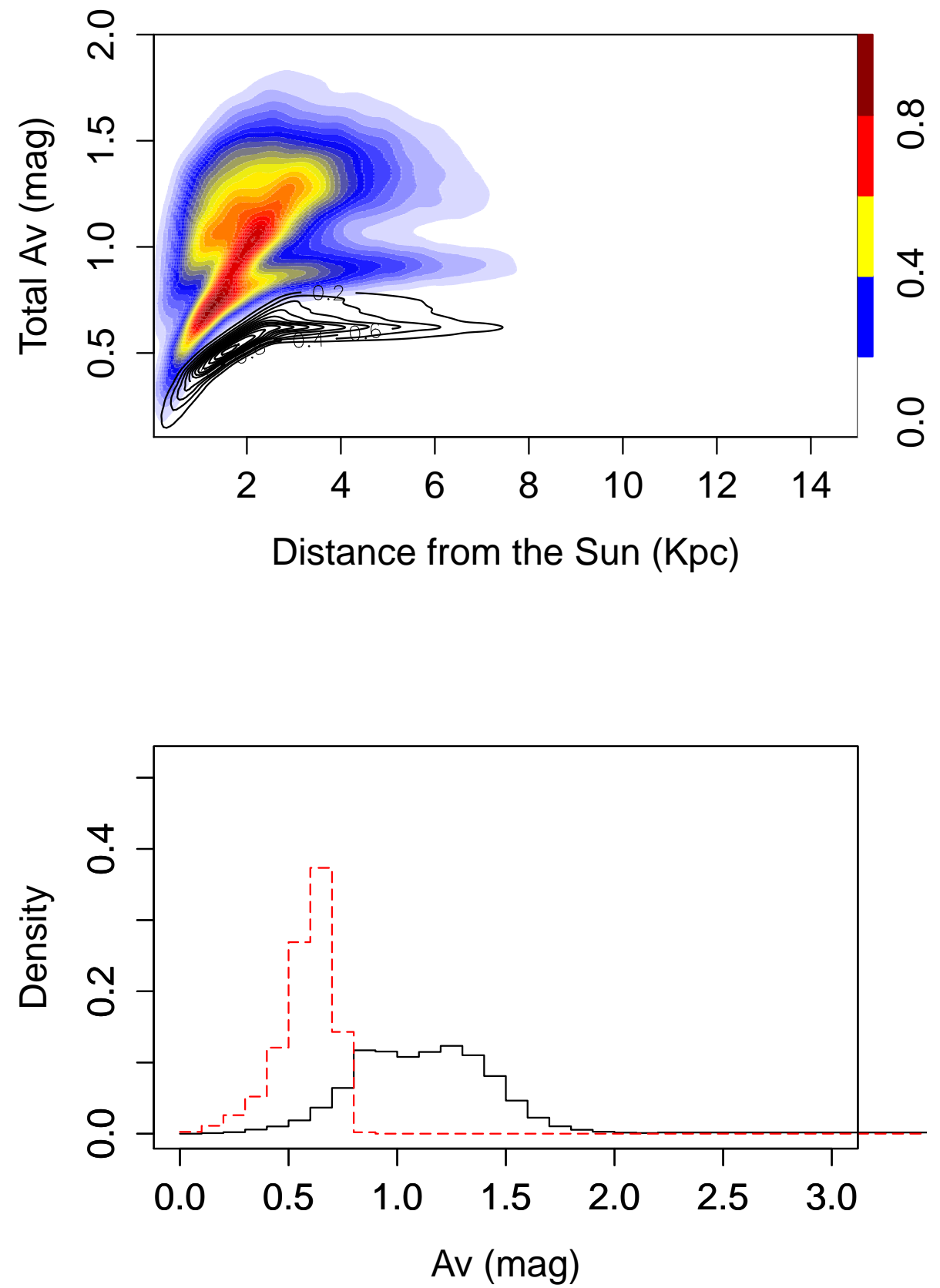

Figure 6.10: Total $\mathrm{A}_{v}$ as a function of the distance from the Sun for field 2537 $(1, b)=\left(110^{\circ}, 10.5^{\circ}\right)$. Density contours are the total $\mathrm{A}_{v}$ after correction and black contours are before the correction. Bottom panel: Total $\mathrm{A}_{v}$ distribution before (red dashed line) and after correction (solid black line). 

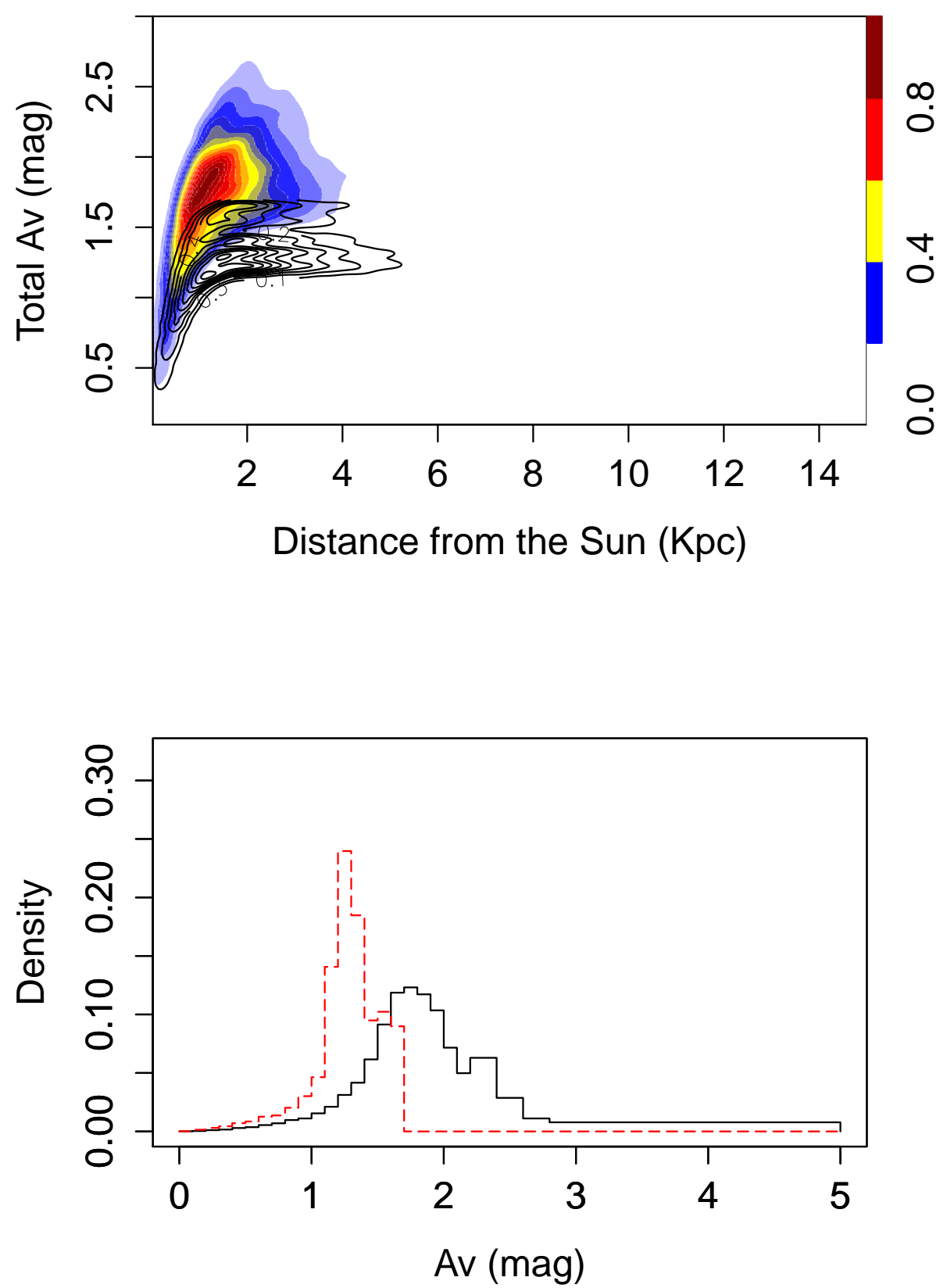

Figure 6.11: Total $\mathrm{A}_{v}$ as a function of the distance from the Sun for field 2538 $(1, b)=\left(110^{\circ}, 16^{\circ}\right)$. Density contours are the total $\mathrm{A}_{v}$ after correction and black contours are before the correction. Bottom panel: Total $\mathrm{A}_{v}$ distribution before (red dashed line) and after correction (solid black line). 

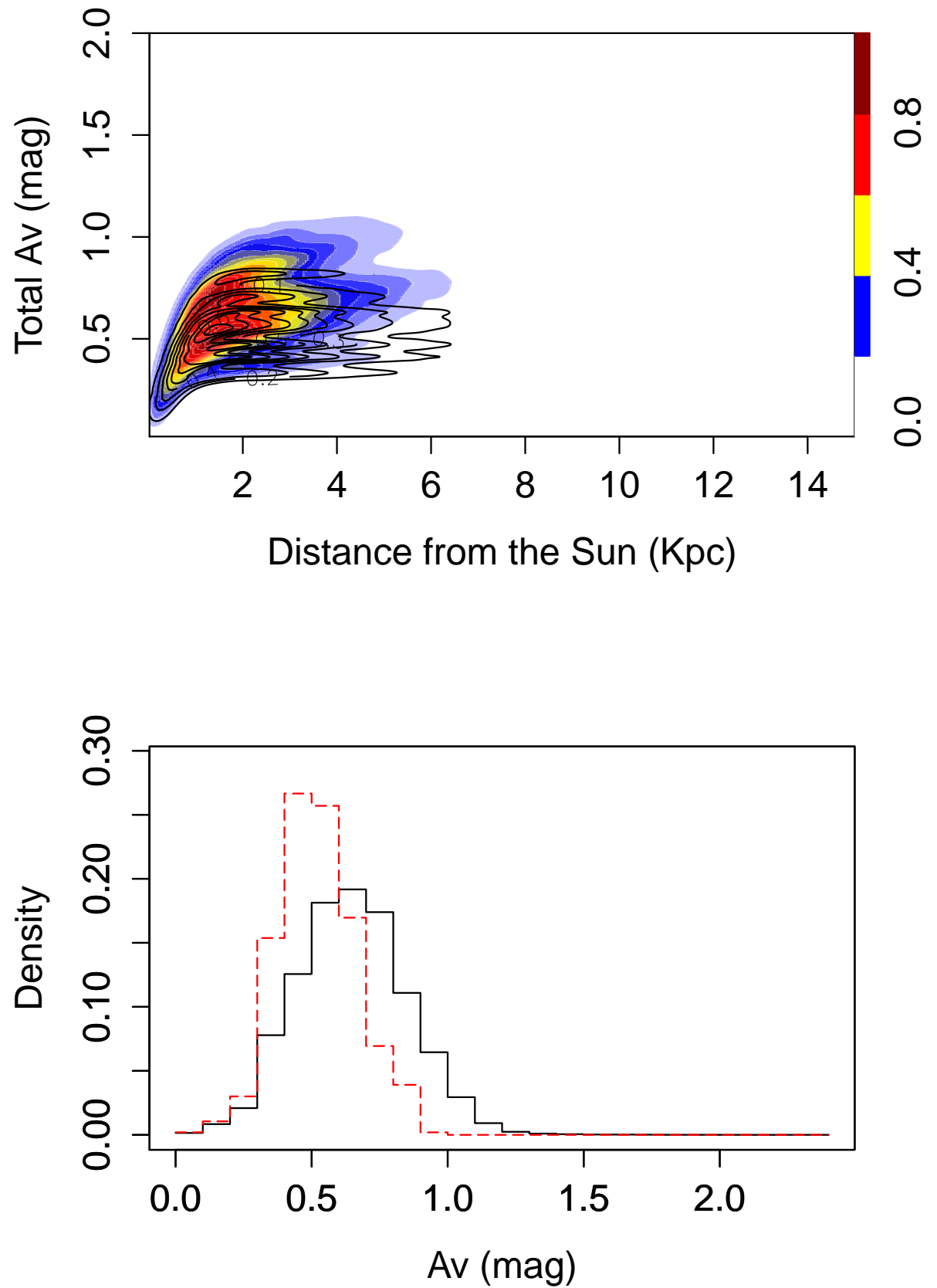

Figure 6.12: Total $A_{v}$ as a function of the distance from the Sun for field $2554(1, b)=\left(94^{\circ}, 14^{\circ}\right)$. Density contours are the total $\mathrm{A}_{v}$ after correction and black contours are before the correction. Bottom panel: Total $\mathrm{A}_{v}$ distribution before (red dashed line) and after correction (solid black line). 

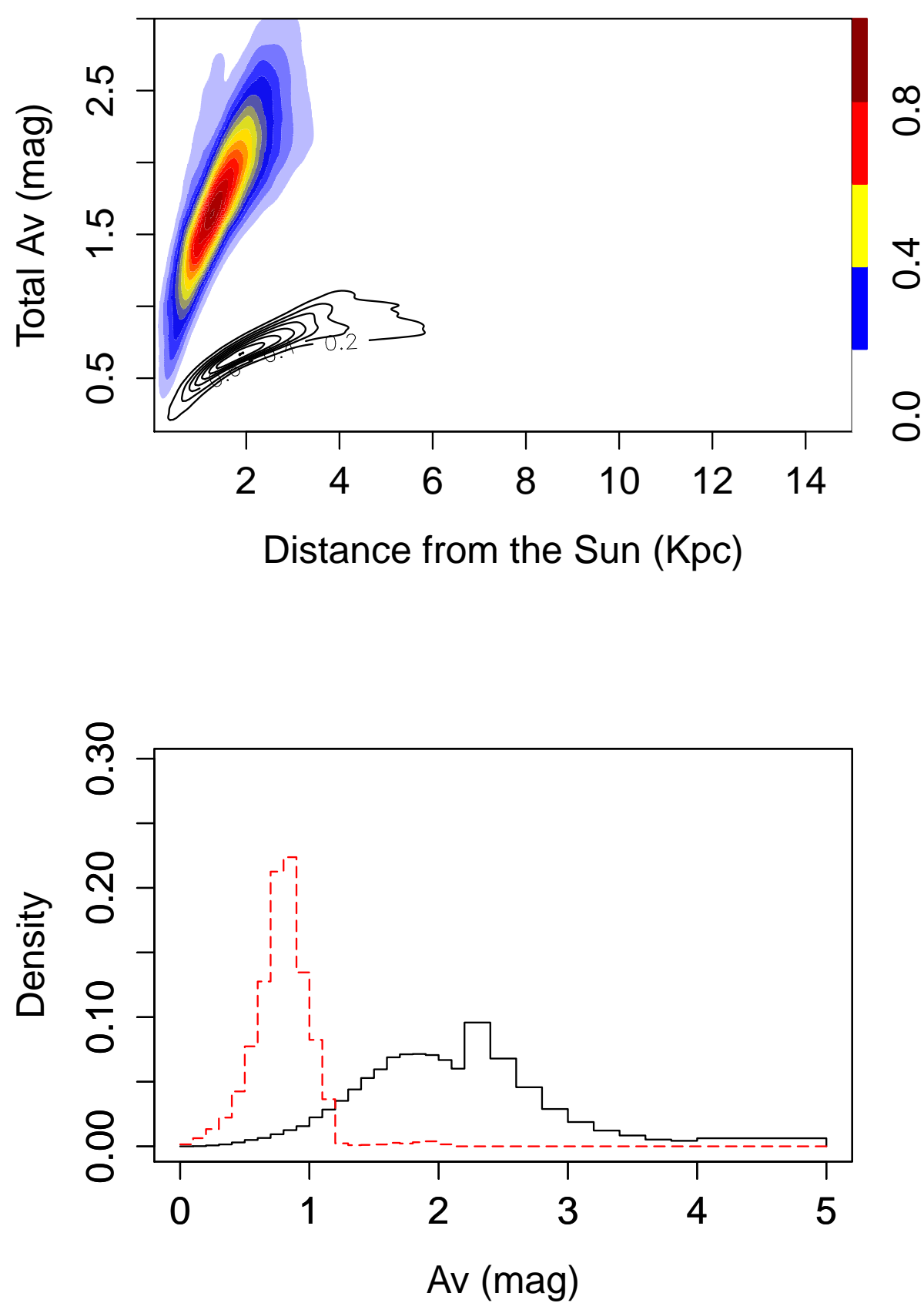

Figure 6.13: Total $A_{v}$ as a function of the distance from the Sun for field $2555(1, b)=\left(94^{\circ}, 8^{\circ}\right)$. Density contours are the total $\mathrm{A}_{v}$ after correction and black contours are before the correction. Bottom panel: Total $\mathrm{A}_{v}$ distribution before (red dashed line) and after correction (solid black line). 

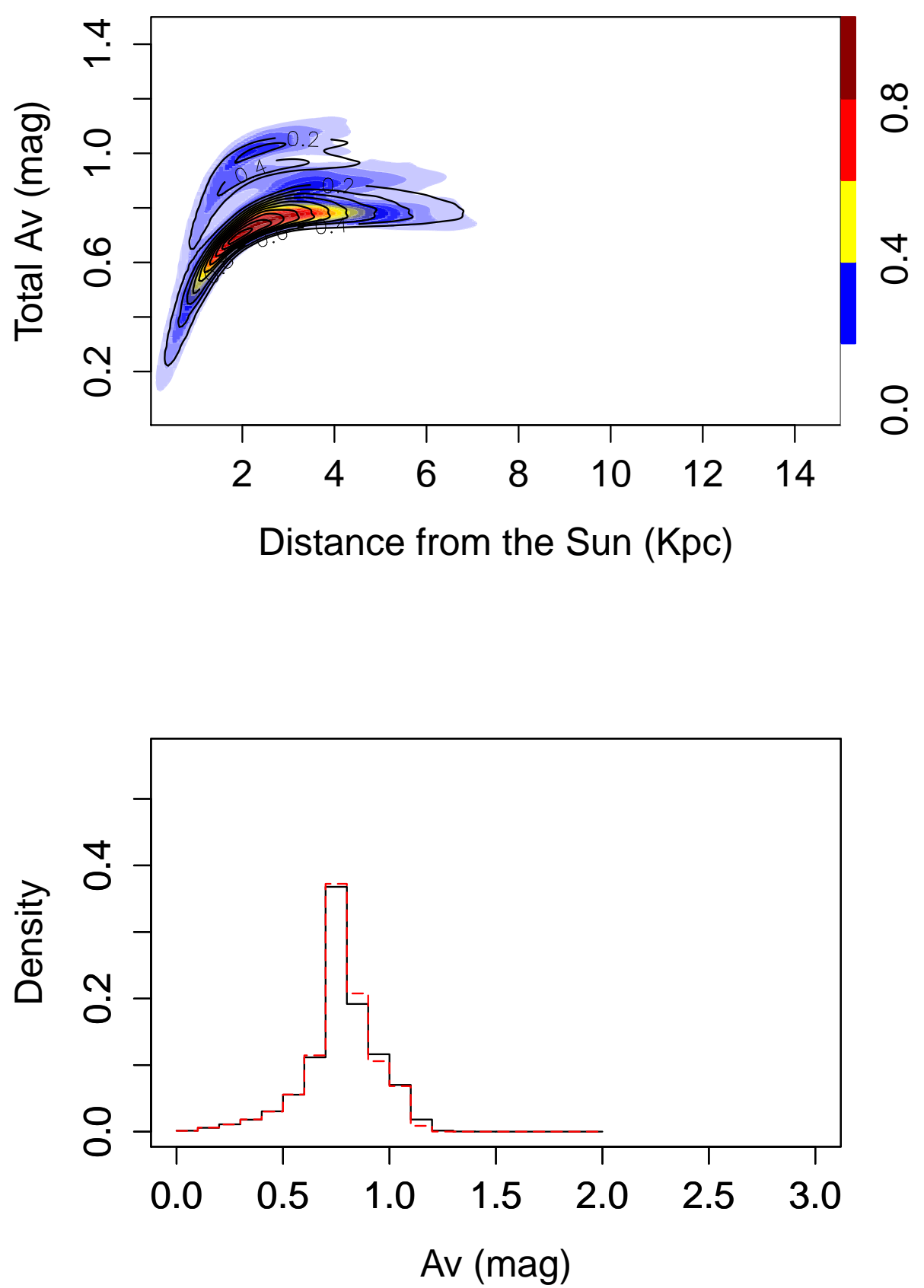

Figure 6.14: Total $A_{v}$ as a function of the distance from the Sun for field $2556(1, b)=\left(94^{\circ},-8^{\circ}\right)$. Density contours are the total $\mathrm{A}_{v}$ after correction and black contours are before the correction. Bottom panel: Total $\mathrm{A}_{v}$ distribution before (red dashed line) and after correction (solid black line). 

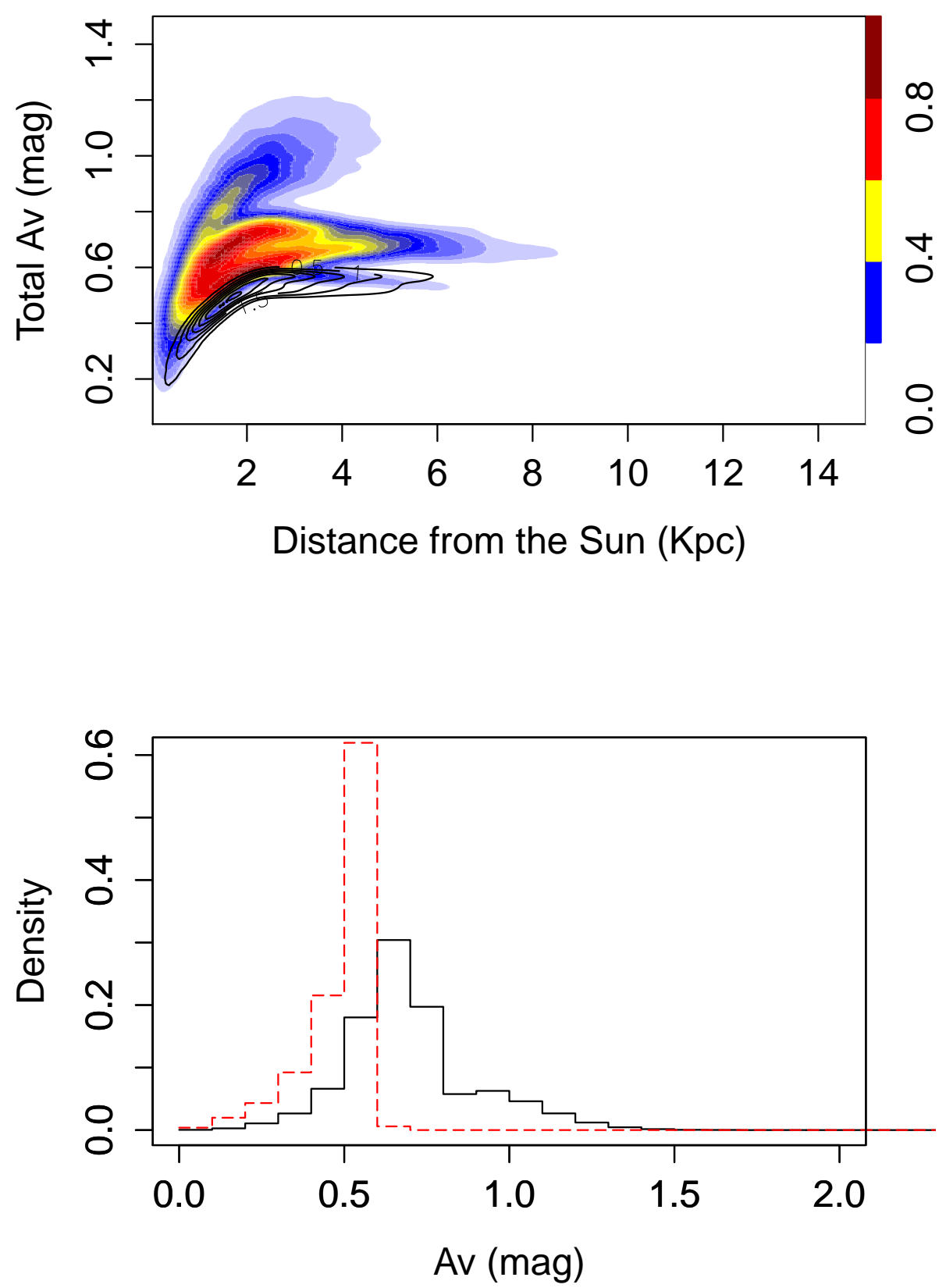

Figure 6.15: Total $\mathrm{A}_{v}$ as a function of the distance from the Sun for field 2668 $(1, b)=\left(187^{\circ},-12^{\circ}\right)$. Density contours are the total $A_{v}$ after correction and black contours are before the correction. Bottom panel: Total $\mathrm{A}_{v}$ distribution before (red dashed line) and after correction (solid black line). 

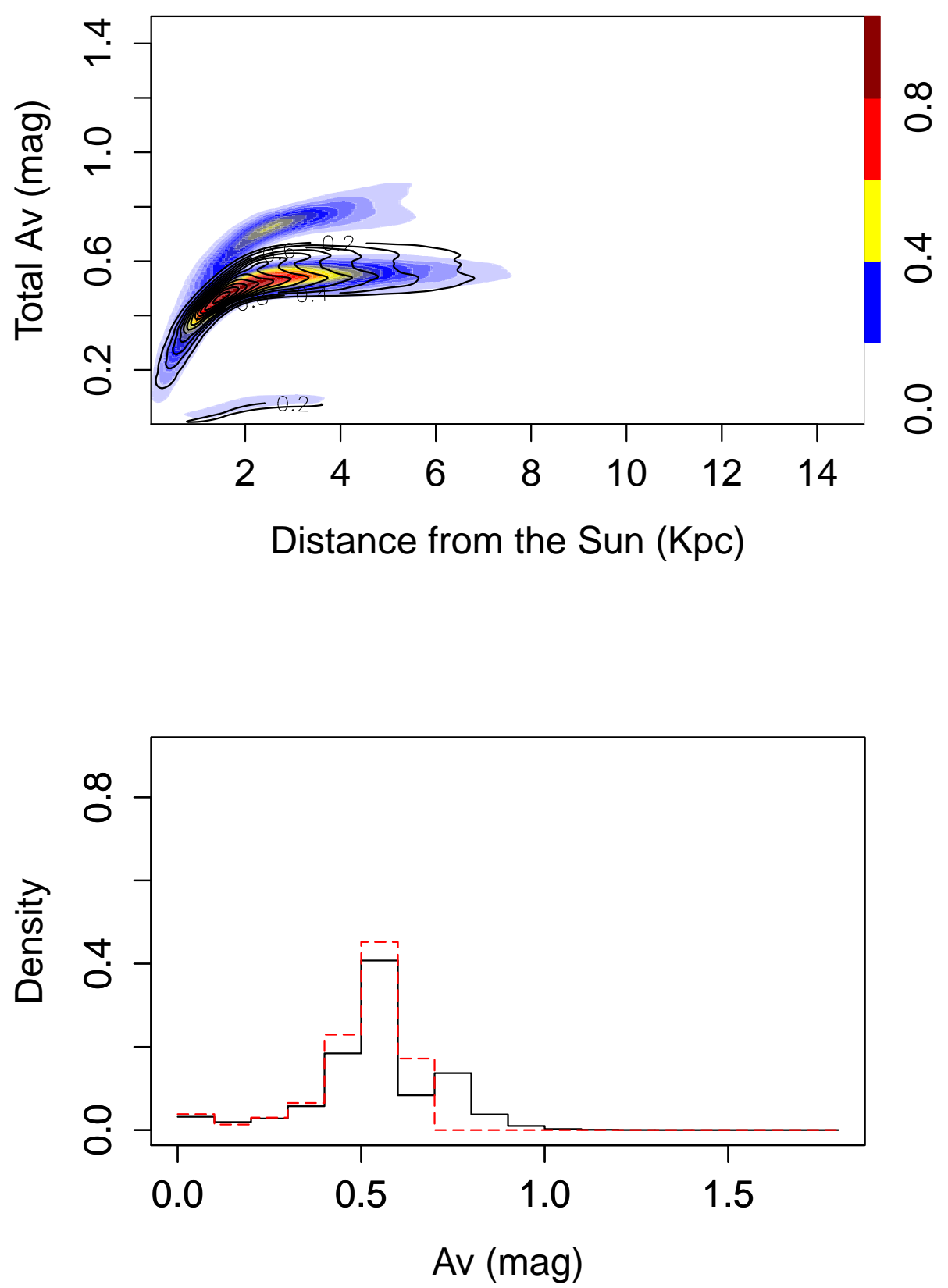

Figure 6.16: Total $A_{v}$ as a function of the distance from the Sun for field $2678(1, b)=\left(187^{\circ},-8^{\circ}\right)$. Density contours are the total $\mathrm{A}_{v}$ after correction and black contours are before the correction. Bottom panel: Total $\mathrm{A}_{v}$ distribution before (red dashed line) and after correction (solid black line). 

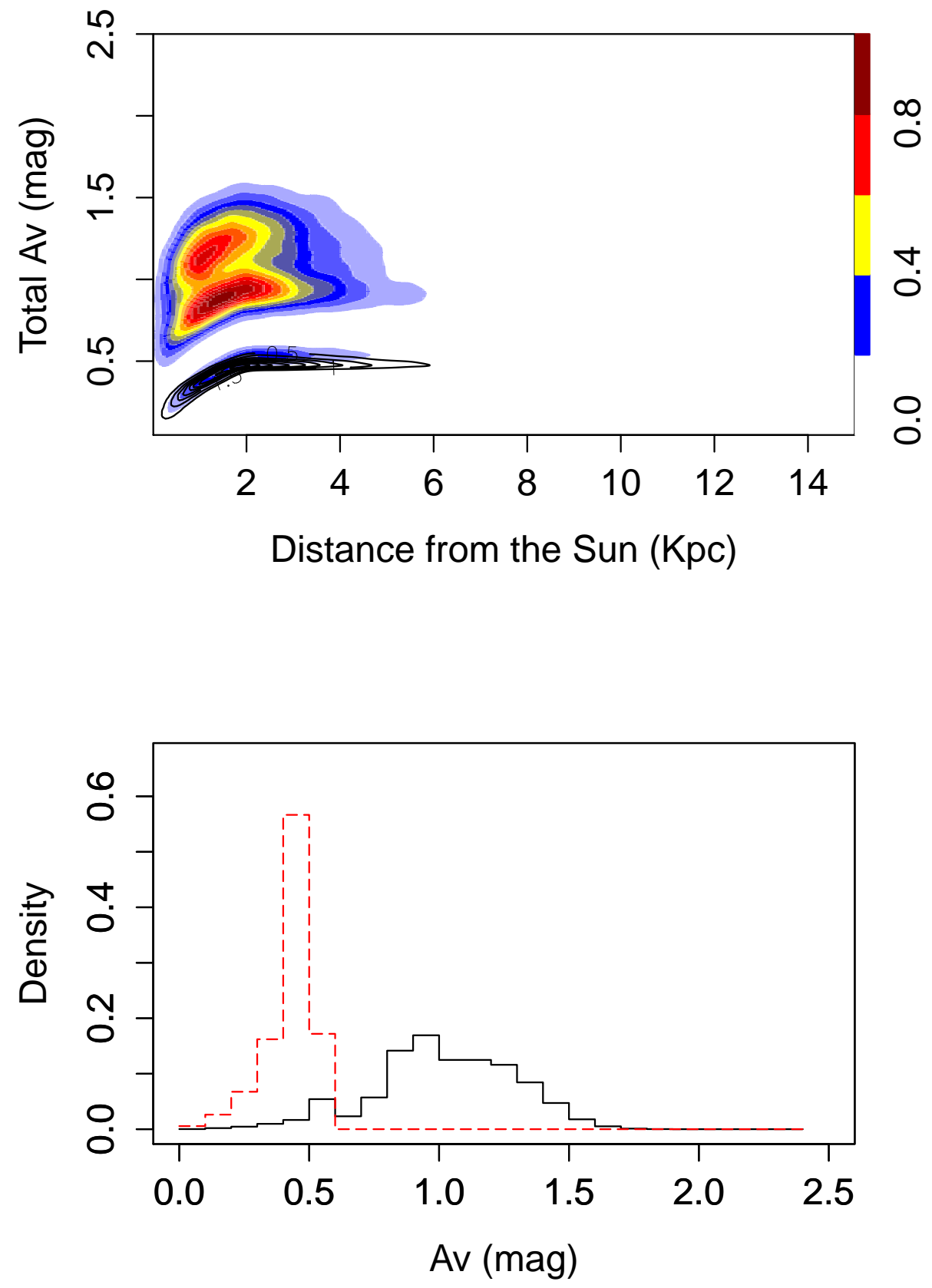

Figure 6.17: Total $\mathrm{A}_{v}$ as a function of the distance from the Sun for field 2681 $(1, b)=\left(178^{\circ},-15^{\circ}\right)$. Density contours are the total $A_{v}$ after correction and black contours are before the correction. Bottom panel: Total $\mathrm{A}_{v}$ distribution before (red dashed line) and after correction (solid black line). 
Table 6.1: Mean, mode, and dispersion of the distribution of the total extinction in $\mathrm{V}$ band $\mathrm{A}_{V}$ before (B) and after (A) correction for each field

\begin{tabular}{ccccccc}
\hline \hline Field & $\operatorname{Mean}_{A}$ & $\operatorname{Mean}_{B}$ & $\operatorname{Mode}_{A}$ & $\operatorname{Mode}_{B}$ & $\sigma_{A}$ & $\sigma_{B}$ \\
\hline 2534 & 0.648 & 0.54 & 0.773 & 0.54 & 0.131 & 0.096 \\
2536 & 0.525 & 0.443 & 0.5 & 0.476 & 0.11 & 0.086 \\
2537 & 1.117 & 0.579 & 0.888 & 0.621 & 0.302 & 0.126 \\
2538 & 1.752 & 1.277 & 1.74 & 1.224 & 0.405 & 0.249 \\
2554 & 0.649 & 0.52 & 0.696 & 0.47 & 0.201 & 0.143 \\
2555 & 1.934 & 0.796 & 1.839 & 0.804 & 0.635 & 0.234 \\
2556 & 0.775 & 0.768 & 0.767 & 0.763 & 0.172 & 0.165 \\
2668 & 0.699 & 0.488 & 0.66 & 0.564 & 0.196 & 0.099 \\
2678 & 0.541 & 0.496 & 0.539 & 0.518 & 0.171 & 0.13 \\
2681 & 1.021 & 0.433 & 0.916 & 0.472 & 0.269 & 0.09 \\
\hline \hline
\end{tabular}

Table 6.2:

Coefficients from the equation 6.2 for the correction to the DS extinction model. The different lines in each field are the different regions in the field where the program has been implemented. The regions were selected, from 1 to 9 , firstly from decreasing galactic latitude and then from increasing galactic longitude.

\begin{tabular}{ccccc}
\hline \hline Field & Region & $b_{0}$ & $a_{1}$ & $\chi^{2}$ \\
\hline 2534 & 1 & $-5.0 \times 10^{-02}$ & $4.5 \times 10^{-02}$ & $6.7 \times 10^{-02}$ \\
2534 & 2 & $-2.0 \times 10^{-02}$ & $5.0 \times 10^{-02}$ & $9.8 \times 10^{-02}$ \\
2534 & 3 & $2.0 \times 10^{-02}$ & $2.0 \times 10^{-02}$ & $7.2 \times 10^{-02}$ \\
2534 & 4 & $1.0 \times 10^{-02}$ & $1.5 \times 10^{-02}$ & 0.10 \\
2534 & 5 & $-1.0 \times 10^{-02}$ & $3.5 \times 10^{-02}$ & $7.9 \times 10^{-02}$ \\
2534 & 6 & $6.0 \times 10^{-02}$ & $1.0 \times 10^{-02}$ & $7.8 \times 10^{-02}$ \\
2534 & 7 & $7.0 \times 10^{-02}$ & $5.0 \times 10^{-03}$ & $8.3 \times 10^{-02}$ \\
2534 & 8 & $1.0 \times 10^{-02}$ & $3.0 \times 10^{-02}$ & 0.11 \\
2534 & 9 & $-3.0 \times 10^{-02}$ & $4.0 \times 10^{-02}$ & $7.5 \times 10^{-02}$ \\
\hline 2536 & 1 & $3.0 \times 10^{-02}$ & $1.5 \times 10^{-02}$ & 0.17 \\
2536 & 2 & $-4.0 \times 10^{-02}$ & $3.5 \times 10^{-02}$ & 0.13 \\
2536 & 3 & $-9.0 \times 10^{-02}$ & $4.5 \times 10^{-02}$ & 0.12 \\
2536 & 4 & $2.0 \times 10^{-02}$ & $1.5 \times 10^{-02}$ & 0.11 \\
2536 & 5 & $-7.0 \times 10^{-02}$ & $3.0 \times 10^{-02}$ & 0.12 \\
2536 & 6 & $2.0 \times 10^{-02}$ & $5.0 \times 10^{-03}$ & $9.1 \times 10^{-02}$ \\
2536 & 7 & $-7.0 \times 10^{-02}$ & $5.0 \times 10^{-02}$ & 0.14 \\
2536 & 8 & $4.0 \times 10^{-02}$ & $1.5 \times 10^{-02}$ & $9.0 \times 10^{-02}$ \\
2536 & 9 & $-5.0 \times 10^{-02}$ & $2.5 \times 10^{-02}$ & $8.7 \times 10^{-02}$
\end{tabular}


continued from previous page

\begin{tabular}{|c|c|c|c|c|}
\hline Field & Region & $b_{0}$ & $a_{1}$ & $\overline{\chi^{2}}$ \\
\hline 2537 & 1 & 0.19 & $-5.0 \times 10^{-03}$ & 0.13 \\
\hline 2537 & 2 & 0.1 & 0.0 & 0.13 \\
\hline 2537 & 3 & 0.18 & $5.0 \times 10^{-03}$ & $5.9 \times 10^{-02}$ \\
\hline 2537 & 4 & 0.34 & $1.5 \times 10^{-02}$ & $4.9 \times 10^{-02}$ \\
\hline 2537 & 5 & 0.14 & $1.5 \times 10^{-02}$ & $7.7 \times 10^{-02}$ \\
\hline 2537 & 6 & 0.17 & $5.0 \times 10^{-03}$ & $8.5 \times 10^{-02}$ \\
\hline 2537 & 7 & 0.29 & $1.0 \times 10^{-02}$ & $7.1 \times 10^{-02}$ \\
\hline 2537 & 8 & 0.27 & $5.0 \times 10^{-03}$ & $7.2 \times 10^{-02}$ \\
\hline 2537 & 9 & 0.22 & $2.0 \times 10^{-02}$ & $4.9 \times 10^{-02}$ \\
\hline 2538 & 1 & $9.0 \times 10^{-02}$ & $6.0 \times 10^{-02}$ & $5.8 \times 10^{-02}$ \\
\hline 2538 & 2 & 0.20 & $3.0 \times 10^{-02}$ & $2.6 \times 10^{-02}$ \\
\hline 2538 & 3 & 0.30 & $-2.0 \times 10^{-02}$ & $2.4 \times 10^{-02}$ \\
\hline 2538 & 4 & 0.40 & $-6.0 \times 10^{-02}$ & $1.6 \times 10^{-02}$ \\
\hline 2538 & 5 & 0.19 & $-2.0 \times 10^{-02}$ & $2.9 \times 10^{-02}$ \\
\hline 2538 & 6 & 0.17 & $2.0 \times 10^{-02}$ & $3.4 \times 10^{-02}$ \\
\hline 2538 & 7 & 0.28 & $5.0 \times 10^{-03}$ & $6.5 \times 10^{-02}$ \\
\hline 2538 & 8 & 0.19 & $1.5 \times 10^{-02}$ & $3.8 \times 10^{-02}$ \\
\hline 2538 & 9 & 0.1 & $5.0 \times 10^{-03}$ & $3.5 \times 10^{-02}$ \\
\hline 2554 & 1 & $6.0 \times 10^{-02}$ & 0.0 & 0.18 \\
\hline 2554 & 2 & $-8.0 \times 10^{-02}$ & $4.5 \times 10^{-02}$ & 0.11 \\
\hline 2554 & 3 & -0.12 & $6.5 \times 10^{-02}$ & 0.12 \\
\hline 2554 & 4 & $3.0 \times 10^{-02}$ & 0.0 & 0.18 \\
\hline 2554 & 5 & $-4.0 \times 10^{-02}$ & $3.0 \times 10^{-02}$ & 0.21 \\
\hline 2554 & 6 & $-3.0 \times 10^{-02}$ & $3.5 \times 10^{-02}$ & 0.11 \\
\hline 2554 & 7 & $-1.0 \times 10^{-02}$ & $2.5 \times 10^{-02}$ & 0.16 \\
\hline 2554 & 8 & $-5.0 \times 10^{-02}$ & $2.5 \times 10^{-02}$ & 0.14 \\
\hline 2554 & 9 & 0.11 & 0.0 & 0.10 \\
\hline 2555 & 1 & 0.42 & $2.5 \times 10^{-02}$ & $4.5 \times 10^{-02}$ \\
\hline 2555 & 2 & 0.46 & $5.0 \times 10^{-03}$ & $4.3 \times 10^{-02}$ \\
\hline 2555 & 3 & 0.68 & $5.0 \times 10^{-02}$ & $6.3 \times 10^{-02}$ \\
\hline 2555 & 4 & 0.40 & $3.0 \times 10^{-02}$ & $2.9 \times 10^{-02}$ \\
\hline 2555 & 5 & 0.43 & $3.0 \times 10^{-02}$ & $6.9 \times 10^{-02}$ \\
\hline 2555 & 6 & 0.47 & $3.5 \times 10^{-02}$ & $5.3 \times 10^{-02}$ \\
\hline 2555 & 7 & 0.41 & $4.0 \times 10^{-02}$ & $4.8 \times 10^{-02}$ \\
\hline 2555 & 8 & 0.45 & $3.0 \times 10^{-02}$ & $3.5 \times 10^{-02}$ \\
\hline 2555 & 9 & 0.51 & $1.5 \times 10^{-02}$ & $3.0 \times 10^{-02}$ \\
\hline 2556 & 1 & $4.0 \times 10^{-02}$ & 0.0 & 0.10 \\
\hline 2556 & 2 & $-2.0 \times 10^{-02}$ & $1.0 \times 10^{-02}$ & 0.13 \\
\hline 2556 & 3 & $-2.0 \times 10^{-02}$ & $1.0 \times 10^{-02}$ & 0.10 \\
\hline 2556 & 4 & $-2.0 \times 10^{-02}$ & $5.0 \times 10^{-03}$ & 0.10 \\
\hline 2556 & 5 & $2.0 \times 10^{-02}$ & 0.0 & $8.6 \times 10^{-02}$ \\
\hline
\end{tabular}




\begin{tabular}{|c|c|c|c|c|}
\hline Field & Region & $\overline{b_{0}}$ & $\overline{a_{1}}$ & $\chi^{2}$ \\
\hline 2556 & 6 & $-4.0 \times 10^{-02}$ & $3.0 \times 10^{-02}$ & 0.10 \\
\hline 2556 & 7 & $7.0 \times 10^{-02}$ & $-1.0 \times 10^{-02}$ & 0.13 \\
\hline 2556 & 8 & $3.0 \times 10^{-02}$ & $-1.0 \times 10^{-02}$ & 0.12 \\
\hline 2556 & 9 & $2.0 \times 10^{-02}$ & $1.0 \times 10^{-02}$ & $5.8 \times 10^{-02}$ \\
\hline 2668 & 1 & $9.0 \times 10^{-02}$ & $-1.0 \times 10^{-02}$ & 0.22 \\
\hline 2668 & 2 & $5.0 \times 10^{-02}$ & $-5.0 \times 10^{-03}$ & 0.11 \\
\hline 2668 & 3 & $-8.0 \times 10^{-02}$ & $2.0 \times 10^{-02}$ & 0.40 \\
\hline 2668 & 4 & $6.0 \times 10^{-02}$ & $-5.0 \times 10^{-03}$ & 0.18 \\
\hline 2668 & 5 & $1.0 \times 10^{-02}$ & 0.0 & 0.33 \\
\hline 2668 & 6 & $6.0 \times 10^{-02}$ & $5.0 \times 10^{-03}$ & 0.18 \\
\hline 2668 & 7 & $2.0 \times 10^{-02}$ & 0.0 & 0.12 \\
\hline 2668 & 8 & 0.12 & $-1.0 \times 10^{-02}$ & 0.21 \\
\hline 2668 & 9 & 0.1 & $5.0 \times 10^{-03}$ & $3.5 \times 10^{-02}$ \\
\hline 2678 & 1 & $-9.0 \times 10^{-02}$ & $1.5 \times 10^{-02}$ & 0.36 \\
\hline 2678 & 2 & $-3.0 \times 10^{-02}$ & $1.5 \times 10^{-02}$ & 0.22 \\
\hline 2678 & 3 & $8.0 \times 10^{-02}$ & $-1.0 \times 10^{-02}$ & 0.11 \\
\hline 2678 & 4 & $-8.0 \times 10^{-02}$ & $1.0 \times 10^{-02}$ & 0.22 \\
\hline 2678 & 5 & $-6.0 \times 10^{-02}$ & $-5.0 \times 10^{-03}$ & 0.23 \\
\hline 2678 & 6 & $1.0 \times 10^{-02}$ & $5.0 \times 10^{-03}$ & 0.15 \\
\hline 2678 & 7 & $-7.0 \times 10^{-02}$ & $1.5 \times 10^{-02}$ & 0.35 \\
\hline 2678 & 8 & $-3.0 \times 10^{-02}$ & $1.0 \times 10^{-02}$ & 0.23 \\
\hline 2678 & 9 & $2.0 \times 10^{-02}$ & $1.0 \times 10^{-02}$ & 0.11 \\
\hline 2681 & 1 & 0.0 & 0.0 & $7.4 \times 10^{-02}$ \\
\hline 2681 & 2 & 0.16 & $3.5 \times 10^{-02}$ & $4.4 \times 10^{-02}$ \\
\hline 2681 & 3 & $8.0 \times 10^{-02}$ & $-1.5 \times 10^{-02}$ & $1.7 \times 10^{-02}$ \\
\hline 2681 & 4 & 0.25 & $1.5 \times 10^{-02}$ & $7.0 \times 10^{-02}$ \\
\hline 2681 & 5 & 0.26 & $-5.0 \times 10^{-03}$ & $8.7 \times 10^{-02}$ \\
\hline 2681 & 6 & 0.20 & $1.5 \times 10^{-02}$ & $6.6 \times 10^{-02}$ \\
\hline 2681 & 7 & $-3.0 \times 10^{-02}$ & $2.5 \times 10^{-02}$ & 0.15 \\
\hline 2681 & 8 & $7.0 \times 10^{-02}$ & $2.0 \times 10^{-02}$ & 0.22 \\
\hline 2681 & 9 & $9.0 \times 10^{-02}$ & $4.0 \times 10^{-02}$ & $7.5 \times 10^{-02}$ \\
\hline
\end{tabular}

Table 6.2 show the results of the fit. We tested the same method using the Marshall et al. (2006) 3D extinction model in the two directions where the maps exist. Results are given in Table 6.3 again with a second order equal to zero.

The smaller coefficients (in special $b_{0}$ ) in Table 6.3 show that Marshall et al. (2006) 3D extinction model values are systematically higher that the ones produced by DS maps for field 2555 therefore Marshall et al. (2006) model is closer to reality. For example one star at a distance of $2 \mathrm{kpc}$ in the first region of the field 2555 will have a correction of $A_{v}=0.47$ with DS maps and of $A_{v}=0.21$ with Marshall maps. Regarding field 2556 the results are equivalent. 
Table 6.3:

Coefficients from the equation 6.2 for the correction to the Marshall extinction model. The different lines in each field are the different regions in the field were the program has been implemented. The regions were selected as in table 6.2

\begin{tabular}{ccccc}
\hline \hline Field & Region & $b_{0}$ & $a_{1}$ & $\chi^{2}$ \\
\hline 2555 & 1 & 0.31 & $-5.0 \times 10^{-02}$ & $4.5 \times 10^{-02}$ \\
2555 & 2 & 0.19 & $-1.5 \times 10^{-02}$ & $6.9 \times 10^{-02}$ \\
2555 & 3 & 0.49 & $-5.0 \times 10^{-03}$ & $3.9 \times 10^{-02}$ \\
2555 & 4 & 0.20 & $2.0 \times 10^{-02}$ & $3.4 \times 10^{-02}$ \\
2555 & 5 & 0.22 & $2.5 \times 10^{-02}$ & $4.5 \times 10^{-02}$ \\
2555 & 6 & 0.30 & $-5.5 \times 10^{-02}$ & $3.2 \times 10^{-02}$ \\
2555 & 7 & 0.31 & $-2.0 \times 10^{-02}$ & $3.5 \times 10^{-02}$ \\
2555 & 8 & 0.19 & $2.0 \times 10^{-02}$ & $3.9 \times 10^{-02}$ \\
2555 & 9 & 0.16 & $3.5 \times 10^{-02}$ & $4.2 \times 10^{-02}$ \\
\hline 2556 & 1 & 0.0 & 0.0 & 0.10 \\
2556 & 2 & $1.0 \times 10^{-02}$ & $5.0 \times 10^{-02}$ & 0.13 \\
2556 & 3 & $9.0 \times 10^{-02}$ & $-2.0 \times 10^{-02}$ & $8.8 \times 10^{-02}$ \\
2556 & 4 & $1.0 \times 10^{-02}$ & $-5.0 \times 10^{-03}$ & $9.9 \times 10^{-02}$ \\
2556 & 5 & $-5.0 \times 10^{-02}$ & $2.5 \times 10^{-02}$ & 0.15 \\
2556 & 6 & $2.0 \times 10^{-02}$ & $5.0 \times 10^{-03}$ & 0.12 \\
2556 & 7 & $3.0 \times 10^{-02}$ & $-5.0 \times 10^{-03}$ & 0.11 \\
2556 & 8 & $4.0 \times 10^{-02}$ & 0.0 & 0.12 \\
2556 & 9 & $-2.0 \times 10^{-02}$ & $1.5 \times 10^{-02}$ & $8.2 \times 10^{-02}$ \\
\hline \hline
\end{tabular}



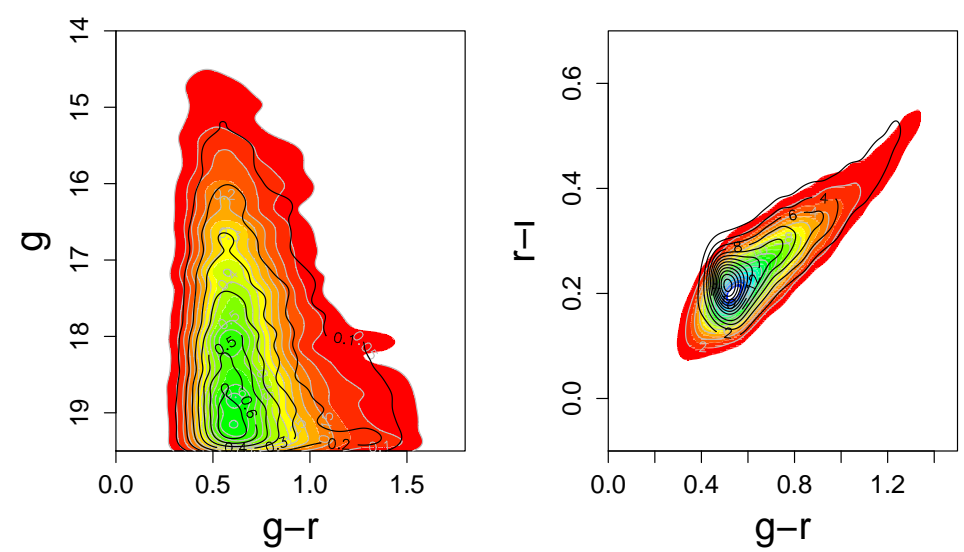

Figure 6.18: Color-magnitude diagram for region one of field $2555(1, b)=\left(94^{\circ}, 8^{\circ}\right)$. Density map along with grey contours are observations. Black contours are simulations.

We then checked the correction using the CMD and CCD. For example 6.18 shows them for field 2555 region 1 . The color peaks in density are the same for simulations and observations and the contours of equal density follow similar patterns. The color-color diagrams have now a maximum density at the same position. The result for each region suggests that the extinction correction is done properly.

\subsection{The $S / N$, proper motions and spectral parameter errors}

We have to compute $\mathrm{S} / \mathrm{N}$ and observational errors from data and apply them to the simulated spectrocopic parameters. The $\mathrm{S} / \mathrm{N}$ ratio is computed by fitting a regression of the $\mathrm{S} / \mathrm{N}$ as a function of the $r$ magnitude from the observations, as shown in figure 6.19 for the plate 2668 , and applying it to simulations. The results of the regression are shown in Table 6.4

A similar procedure was made to simulate the error on the metallicity, effective temperature, $\log \mathrm{g}$ and radial velocity as a function of $\mathrm{g}$ magnitude (we tried as a function of $\mathrm{S} / \mathrm{N}$ but the relation was less robust). Figure 6.20 shows the relation for each of the parameters for plate 2699. Discussions with the SEGUE collaborations suggested to use instead a fix value for all magnitudes, otherwise the errors are underestimated, therefore we assume errors of 0.23 dex, $180 \mathrm{~K}$ and 0.24 dex respectively for metallicity, effective temperature and $\log \mathrm{g}$ (Smolinski et al. 2011). For the radial velocity we use the above method and the relations are given in Table 6.5. The proper motions errors are 4.0 and $3.0 \mathrm{mas} / \mathrm{yr}$, respectively for right ascension and declination (Munn et al. 2004). 


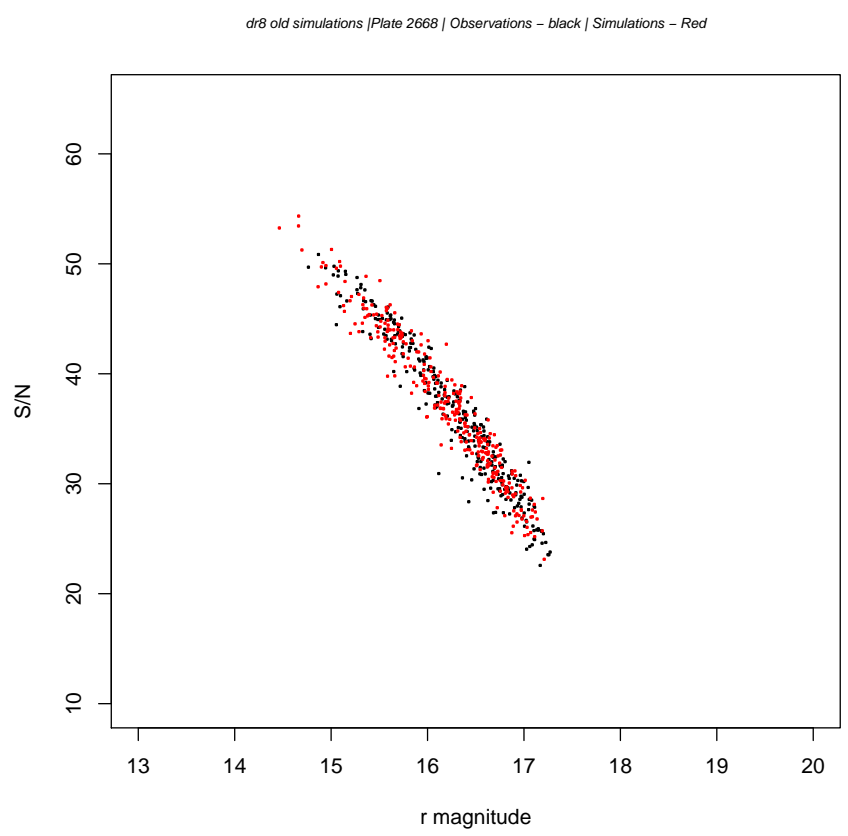

Figure 6.19: $\mathrm{S} / \mathrm{N}$ as a function of the $\mathrm{r}$ magnitude for the plate 2668 . Black points are observations and red points are simulations after applying the fit.
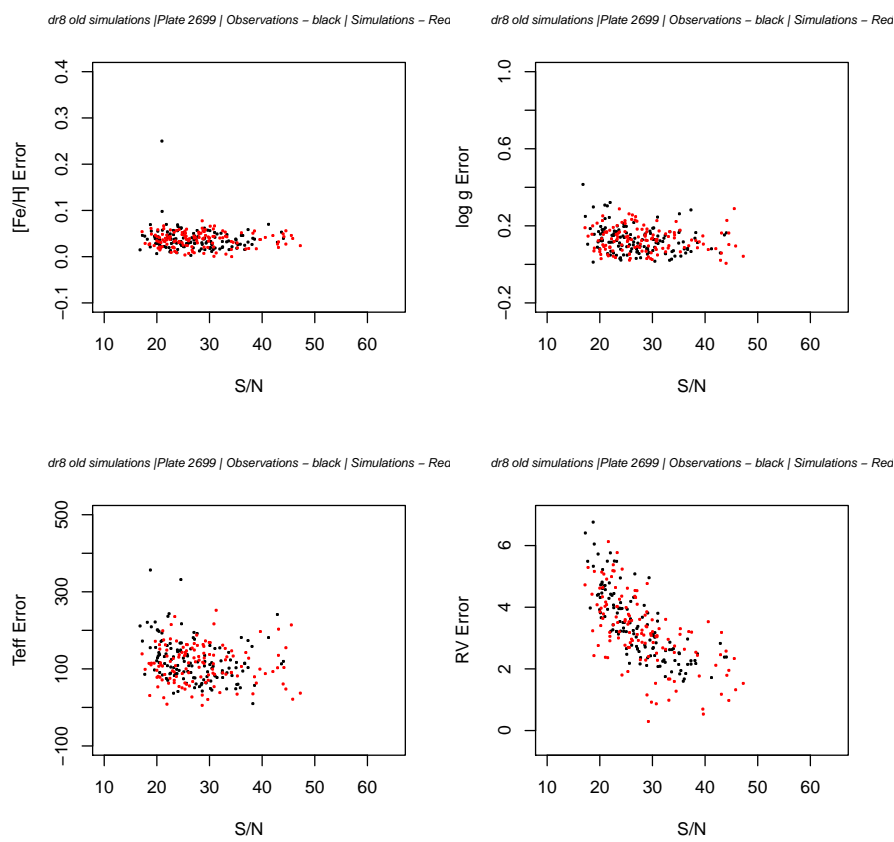

Figure 6.20: Parameter errors as a function of the $\mathrm{S} / \mathrm{N}$ for plate 2699. Top left panel shows the metallicity error, bottom left: effective temperature, top right: gravity, bottom right: radial velocity. 
Table 6.4:

Regression laws for $\mathrm{S} / \mathrm{N}$ as a function of $\mathrm{r}$ magnitude.

\begin{tabular}{cccc}
\hline \hline Plate & Intercept & First order coeff & Second order coeff \\
\hline 2534 & -140.105 & 26.723 & -1.031 \\
2542 & 725.417 & -59.799 & 1.177 \\
2536 & -96.420 & 28.062 & -1.227 \\
2544 & 905.727 & -81.267 & 1.810 \\
2537 & 470.189 & -38.371 & 0.701 \\
2545 & 1106.387 & -101.715 & 2.323 \\
2538 & -216.600 & 43.937 & -1.752 \\
2546 & 511.941 & -38.883 & 0.657 \\
2554 & 180.188 & -6.374 & -0.161 \\
2564 & 1084.935 & -96.781 & 2.135 \\
2555 & -85.562 & 26.813 & -1.201 \\
2565 & 337.625 & -16.736 & -0.031 \\
2556 & -95.128 & 27.533 & -1.187 \\
2566 & 563.282 & -43.077 & 0.747 \\
2668 & -106.999 & 29.211 & -1.253 \\
2672 & 1139.407 & -102.340 & 2.273 \\
2678 & -62.228 & 23.342 & -1.064 \\
2696 & 1306.661 & -121.831 & 2.830 \\
2681 & 714.959 & -62.058 & 1.274 \\
2699 & 321.883 & -21.236 & 0.255 \\
\hline \hline
\end{tabular}


Table 6.5:

Regression laws for radial velocity errors as function of $g$ magnitude for each plates.

\begin{tabular}{lccc}
\hline \hline Parameter & Plate & Intercept & First order coeff \\
\hline RV (Bright) & 2536 & -5.3942 & 0.4116 \\
RV (Faint) & 2544 & -23.6218 & 1.4077 \\
RV (Bright) & 2537 & -22.3452 & 1.4876 \\
RV (Faint) & 2545 & -34.5212 & 2.0194 \\
RV (Bright) & 2538 & -3.7817 & 0.3152 \\
RV (Faint) & 2546 & -18.4919 & 1.1361 \\
RV (Bright) & 2554 & -6.0450 & 0.4490 \\
RV (Faint) & 2564 & -14.4728 & 0.8912 \\
RV (Bright) & 2555 & -1.7838 & 0.2121 \\
RV (Faint) & 2565 & -15.8153 & 0.9916 \\
RV (Bright) & 2556 & -6.1129 & 0.4423 \\
RV (Faint) & 2566 & -11.3316 & 0.7321 \\
RV (Bright) & 2668 & -6.1456 & 0.4696 \\
RV (Faint) & 2672 & -21.7431 & 1.3253 \\
\hline \hline
\end{tabular}

\subsection{Selection Sample}

We have selected two stellar categories from the spectroscopic sample: Main Sequence Turnoff stars and K giants. We have selected Main Sequence Turnoff stars because they enable the study of gradients and chemical distribution for main sequence stars at large distances. We selected $\mathrm{K}$ giants because they were assigned around 300 fibers by plate are highly luminous stars and relatively old stars which allow to study the galactic evolution and chemical distributions at large distances. In the following sections we describe the selection sample for each of targeted categories.

\subsubsection{Main Sequence turnoff selection}

For the Main Sequence turnoff (MSTO) selection we have followed Cheng et al. (2012b) selection sample, which is summarized as follows:

1. Remove all stars with $g>20$ and $i<14.2$, to ensure high quality spectroscopy.

2. Remove regions of highest extinction to maximize the number of high quality spectroscopy. For each half of the plate we estimate the 75 th percentile of the $\mathrm{E}(\mathrm{B}-\mathrm{V})$ dis- 
tribution (Schlegel et al. (1998)), and remove the objects where $\mathrm{E}(\mathrm{B}-\mathrm{V})$ is larger than the higher of the 75th percentile values.

3. Apply the SFD extinction and examine $(g-r)_{S F D}$ distribution in bins of $g_{S F D}(1 \mathrm{mag}$ wide)

4. For each distribution, find the peak $(g-r)_{S F D}$ of the MSTO, in each $g$ bin.

5. Determine the $(g-r)_{\text {half-max }}$ (color distribution is half of the maximum value) on the blue side.

6. The red cut for each bin is defined as $(g-r)_{\text {red-cut }}=(g-r)_{\text {peak }}+(g-r)_{\text {half-max }}+0.25$

7. Fit a line to $(g-r)_{r e d-c u t}$ as a function of the mean $g_{S F D}$ of all stars in the bin.

8. The stars present in the blue side of the line will be considered suitable candidates for being main sequence turnoff stars.

The selection is applied on the simulated catalogue following the same method. We bin the samples in bins of g magnitude (0.1) and g-r color (0.05) and select from the simulated catalogue the same number of stars in magnitude and color present in the observed catalogue. The total number of stars in both catalogs is 5368 .

\subsubsection{K giants selection}

For the $\mathrm{K}$ giants the selection criteria described in the SDSS/SEGUE selection criteria page is followed (Yanny et al. 2009). For the $\mathrm{K}$ giants criteria, the $\mathrm{g}$ magnitude has to be lower than 19.0, the color cut is $0.55<(g-r)<0.9$ and the total proper motion $P M_{\text {Total }}<11$ mas $/ y r$. Applying these constrains to the observed sample a final catalog of selected $\mathrm{K}$ giants is obtained. An equal procedure is done to the simulated catalog having previously corrected the photometry with the DS extinction model and the extinction correction program. Then we use the above described binning in color and magnitude in order to select the same number of stars and CMD distribution in the simulations as in the data. 


\section{Chapter 7}

\section{Photometric results}

\subsection{Comparison between observations and simulations}

In the following sections we qualitatively compare magnitude, color and proper motion distributions from simulations (sim) and observations (obs) from SEGUE fields. We have grouped the fields by similar longitudes or latitudes that are described in the begining of each section.

\subsubsection{Fields 2534 and 2536}

These two fields are located at a different galactic longitudes $\left(l_{2534}=50^{\circ} \& l_{2536}=70^{\circ}\right)$ but the galactic latitude is the same $\left(b_{2534 / 2536}=14^{\circ}\right)$. When we compare observations and simulations for these two fields the results are quite similar. These directions suffer from a small amount of extinction across the field and the simulations reproduce well the star counts for each region individually and for the total sample. In figure 7.1 it is shown the magnitude distribution along with the number of stars in each catalog and the ratio between both for each of the regions of the field 2536. 7.2 presents color distributions along with the mode in each catalog for each of the regions of the field 2536 . Field 2534 has a similar behaviour. The ratio of the number of stars in simulations to the number of stars in the observations remains constant for each region and there is a good agreement between observations and simulations. The modes of the distribution in each catalog agrees for each region.

Figure 7.3 presents the parameters distributions for the all area of field 2536. The two top histograms show a good agreement between observations and simulations for the magnitude and color distributions. The two proper motion ( 1 and $b$ ) histograms show that observations, for the fields in question, have a peak around 0 what is not represented in the simulations for the 1 component of the proper motion. This shift is more proeminent in these two fields but can be also seen in other directions. This is out of the scope of this thesis and will be the subject of another study. Figure 7.4 show that these directions have a large contribution from the thick 

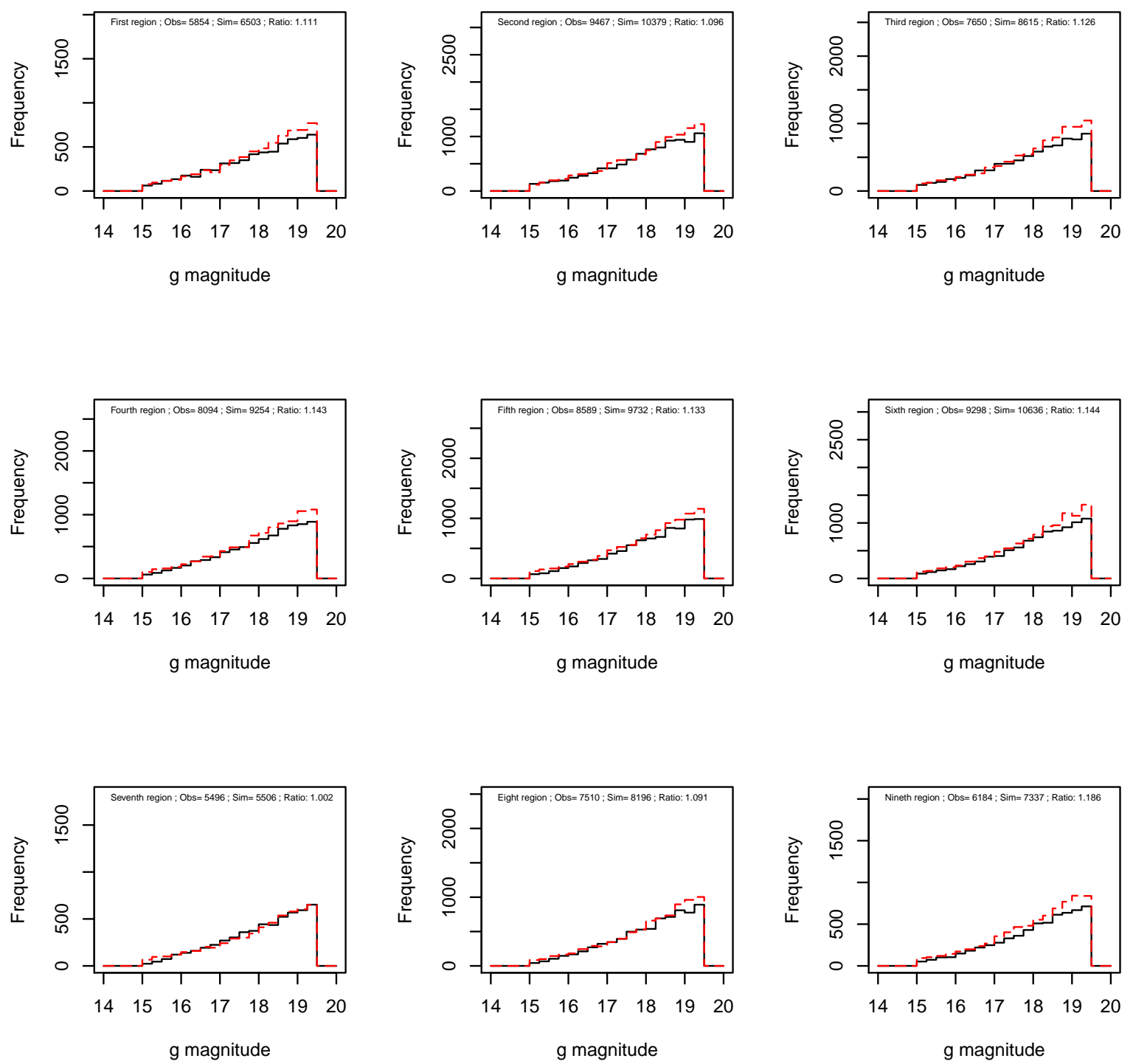

Figure 7.1: Magnitude distribution for each individual region of the field 2536. The black histograms are observations and the red histograms are simulations. 

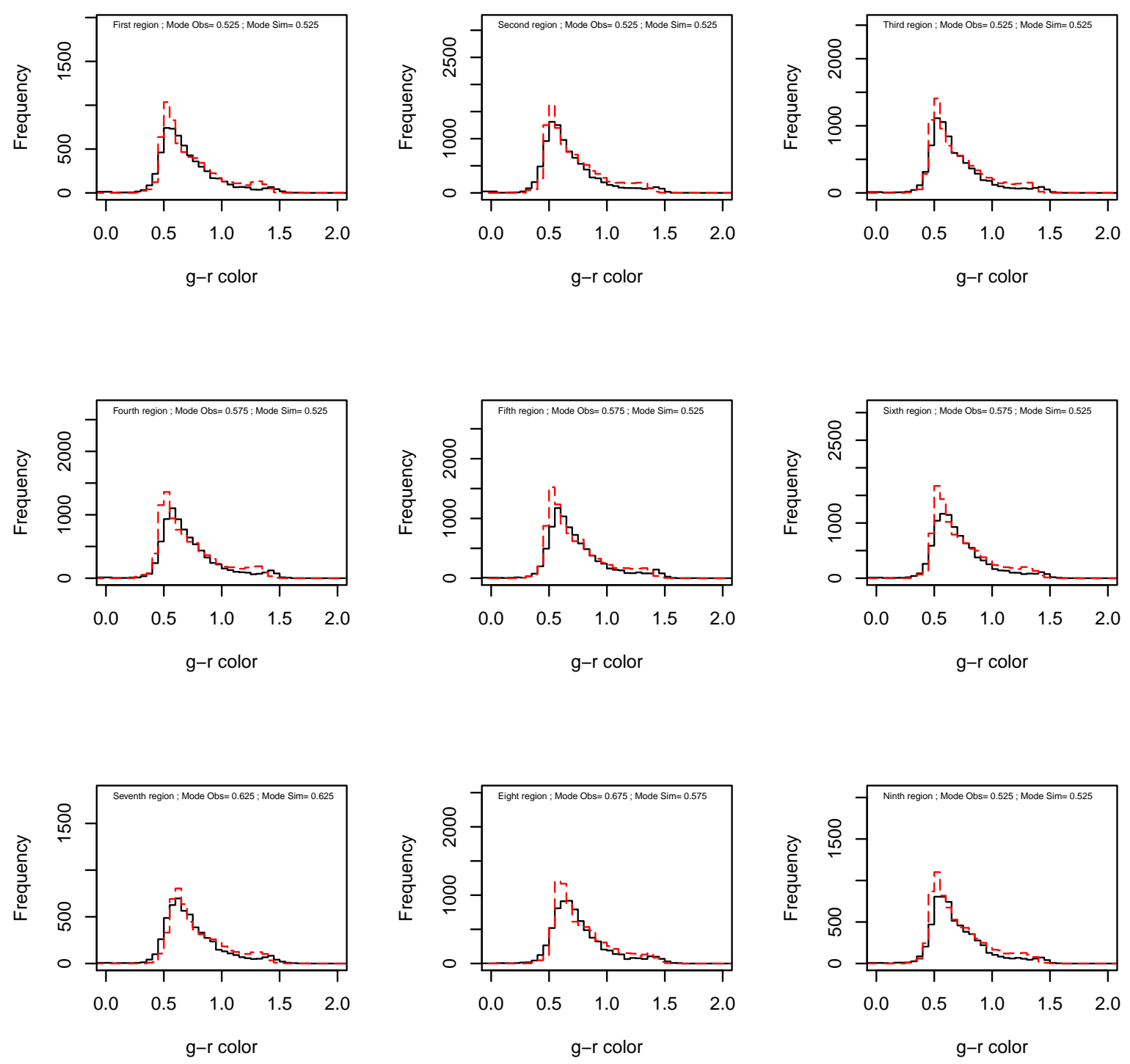

Figure 7.2: Color distribution for each individual region of the field 2536. The black histograms are observations and the red histograms are simulations. 
disc. 

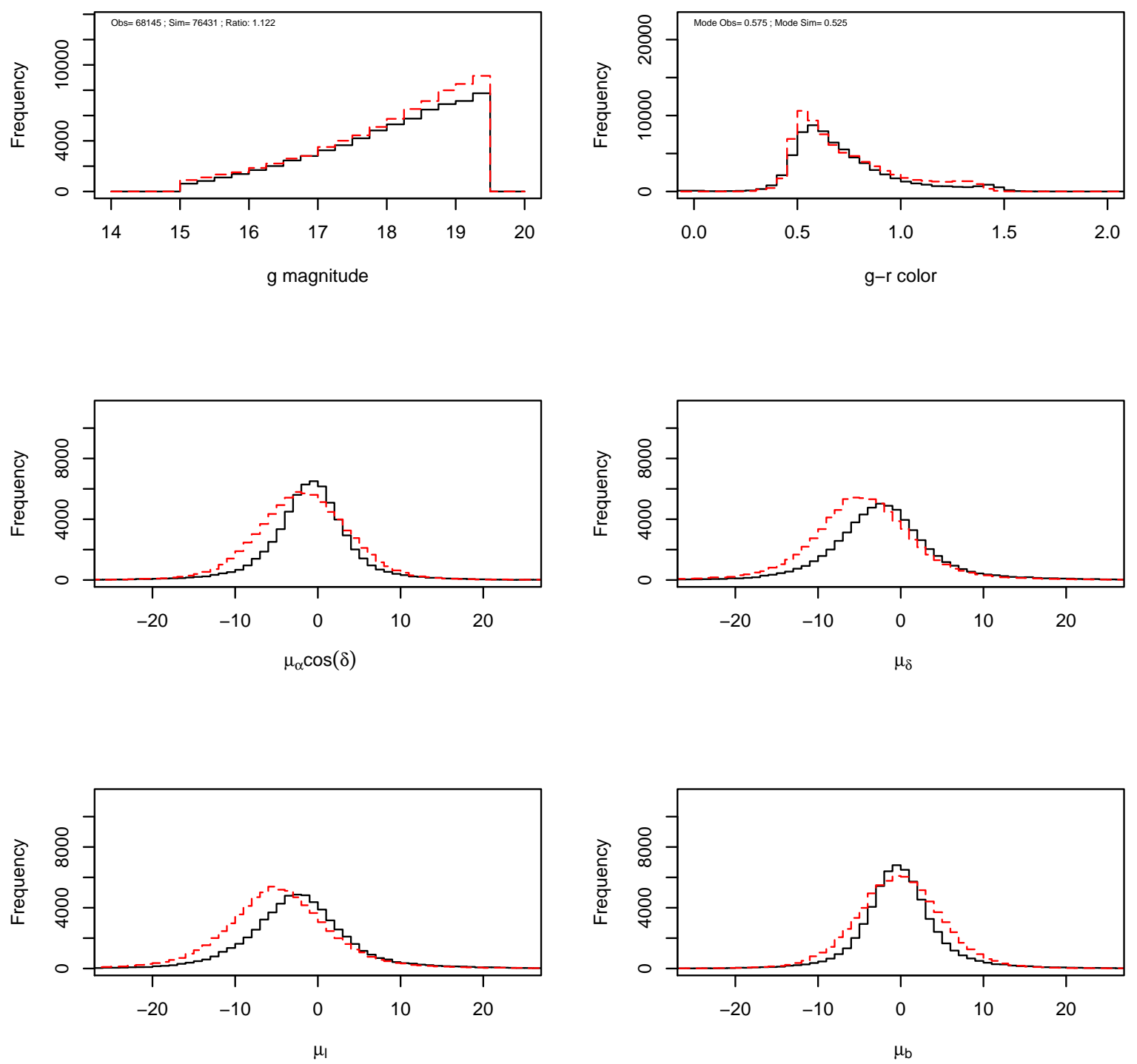

Figure 7.3: Magnitude, color (top panels) and proper motion distributions (middle and bottom panels) for field 2536. Black histograms are observations and the red histograms are simulations. 


\subsubsection{Fields 2537 and 2538}

These two fields are located at an equal galactic longitude $\left(l_{2537 / 2538}=110^{\circ}\right)$ but the galactic latitude is different $\left(b_{2537}=10.5^{\circ} \& b_{2538}=16^{\circ}\right)$. These fields suffer from a large extinction $\left(\mathrm{A}_{V}\right)$ across the field (1.0 - $\left.2.5 \mathrm{mag}\right)$. Magnitude distributions along with the number of stars and the ratio for each region of field 2537 are presented in figure 7.5. Color distributions along with the modes for each region of field 2537 can be observed in figure 7.6. The simulated distributions are in agreement observations (ratio is close to unity) and the color distributions have the same color peak.

Figure 7.7 presents the whole field distributions for magnitude, color and proper motions. The simulated proper motion distribution in the $\mathrm{mu}_{b}$ component has the same peak that observations but the dispersion is too large. The proper motion along longitude in the simulation is shifted with regards to the observation in a field which is dominated by the thin disc (Figure 7.8).

Magnitude distributions along with the number of stars and the ratio for each region of field 2538 are presented in figure 7.9. Color distributions along with the modes for each region of field 2538 can be observed in figure 7.10. The agreement between observations and simulations is not the best, but the ratio (0.7-0.87) between simulations and observations change significantly across the different regions of the field. For each region the color peak is reproduced but regions with larger extinction show larger shifts in color between both distributions.

The number of stars difference in fields 2538 is visible in figure 7.11 Both components of proper motions are slightly shifted and the dispersion is too large probably because we overestimate proper motion errors. In figure 7.12 the thick disc component is comparable to the thin disc at faint magnitudes. The difference in star counts may be explained by the warp of the disc. Figure 7.12 shows that the field is dominated by the thin disc and the shift present in the simulations is due to the thin disc component.

The disagreement in the number of stars for this fields is an indication that the shape of the thick disc may be still subject of improvement by changing the scale height or lenght or by improving the warp and flare modeling among other possibilities. 

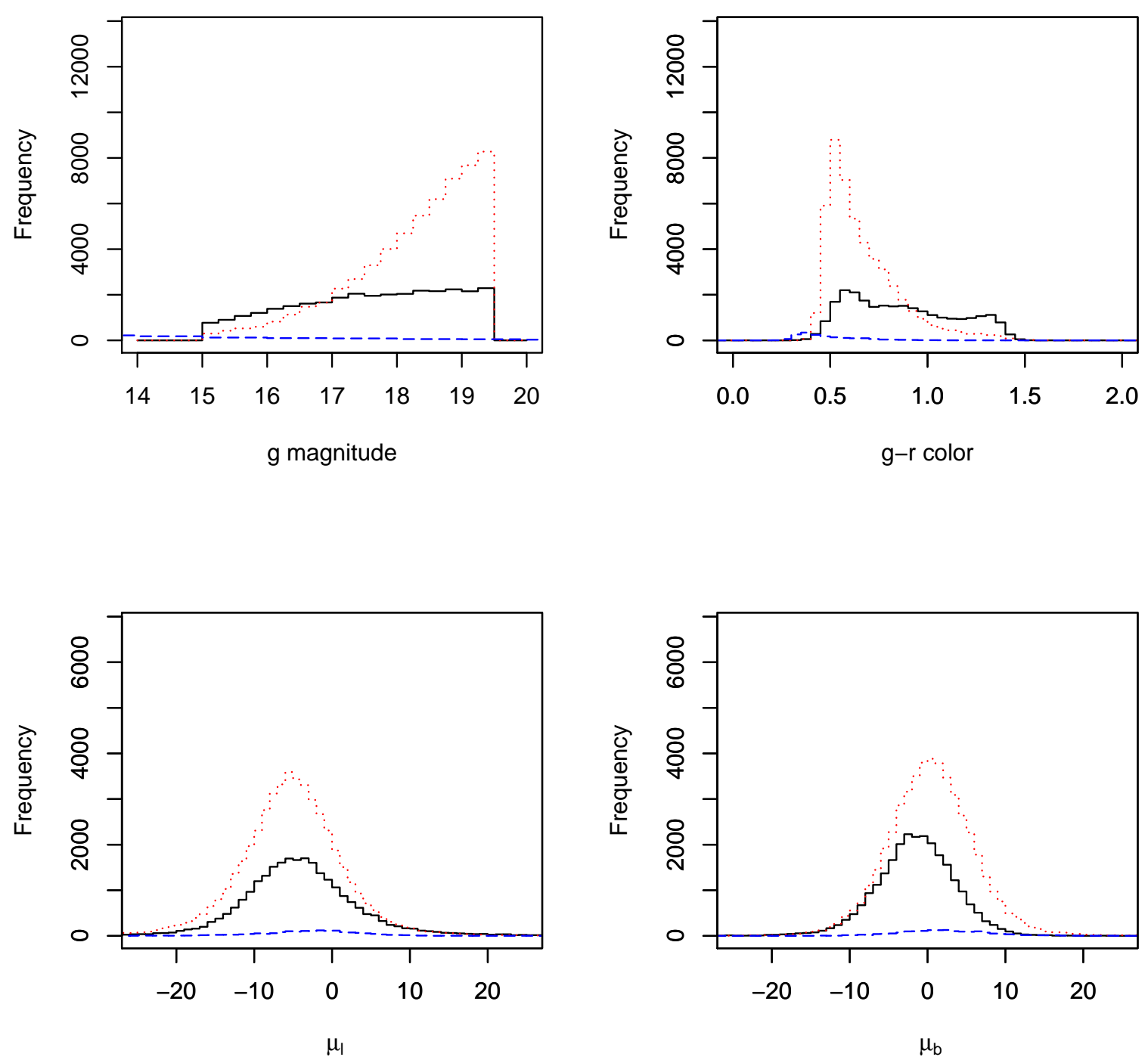

Figure 7.4: Simulated magnitude, color (top panels) and proper motion distributions (bottom panels) separated by populations for field 2536. Thin disc stars: Black solid line; Thick disc stars: Red dotted line; Halo stars: Blue dashed line. 

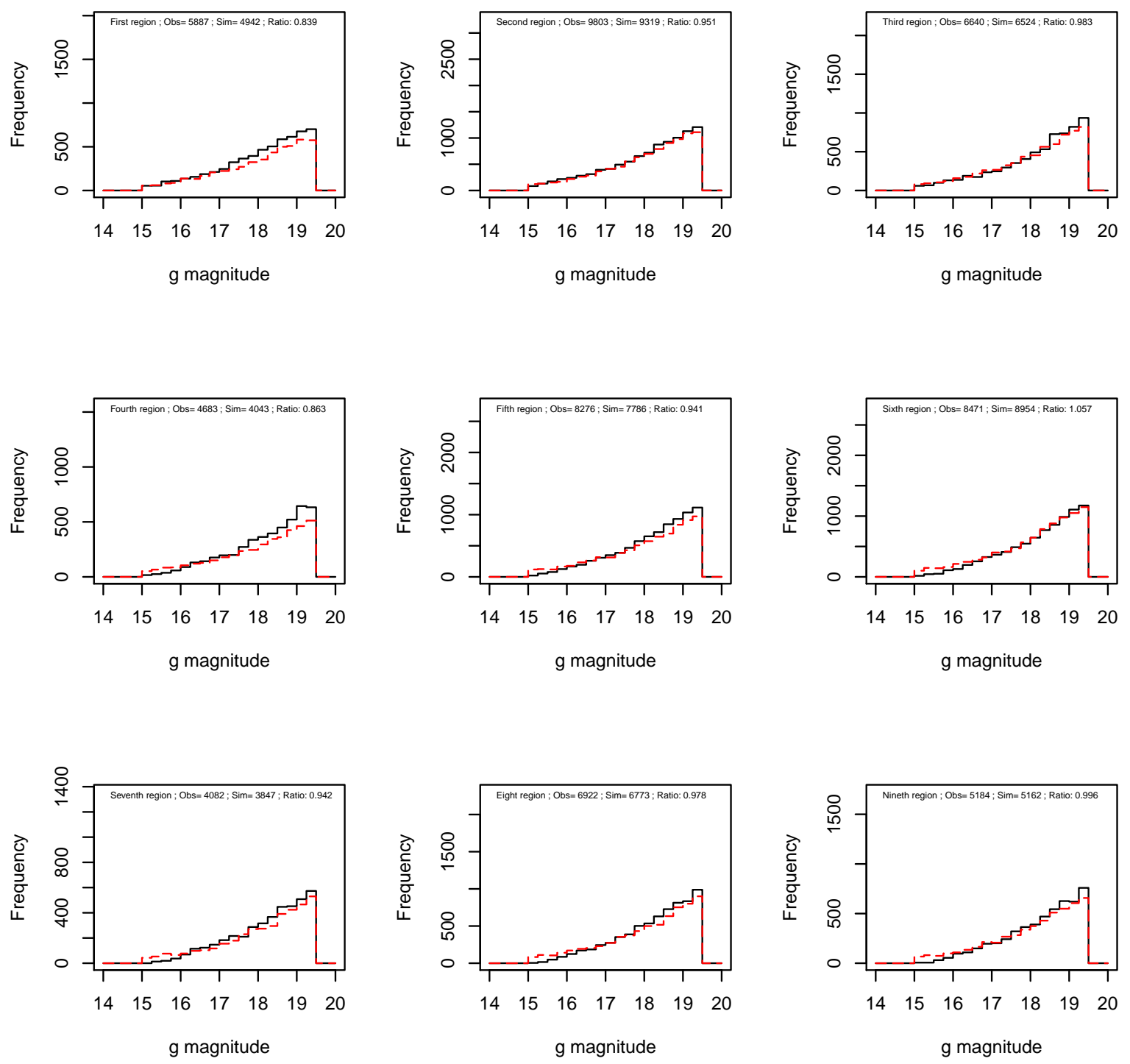

Figure 7.5: Magnitude distribution for each individual region of the field 2537. The black histograms are observations and the red histograms are simulations. 

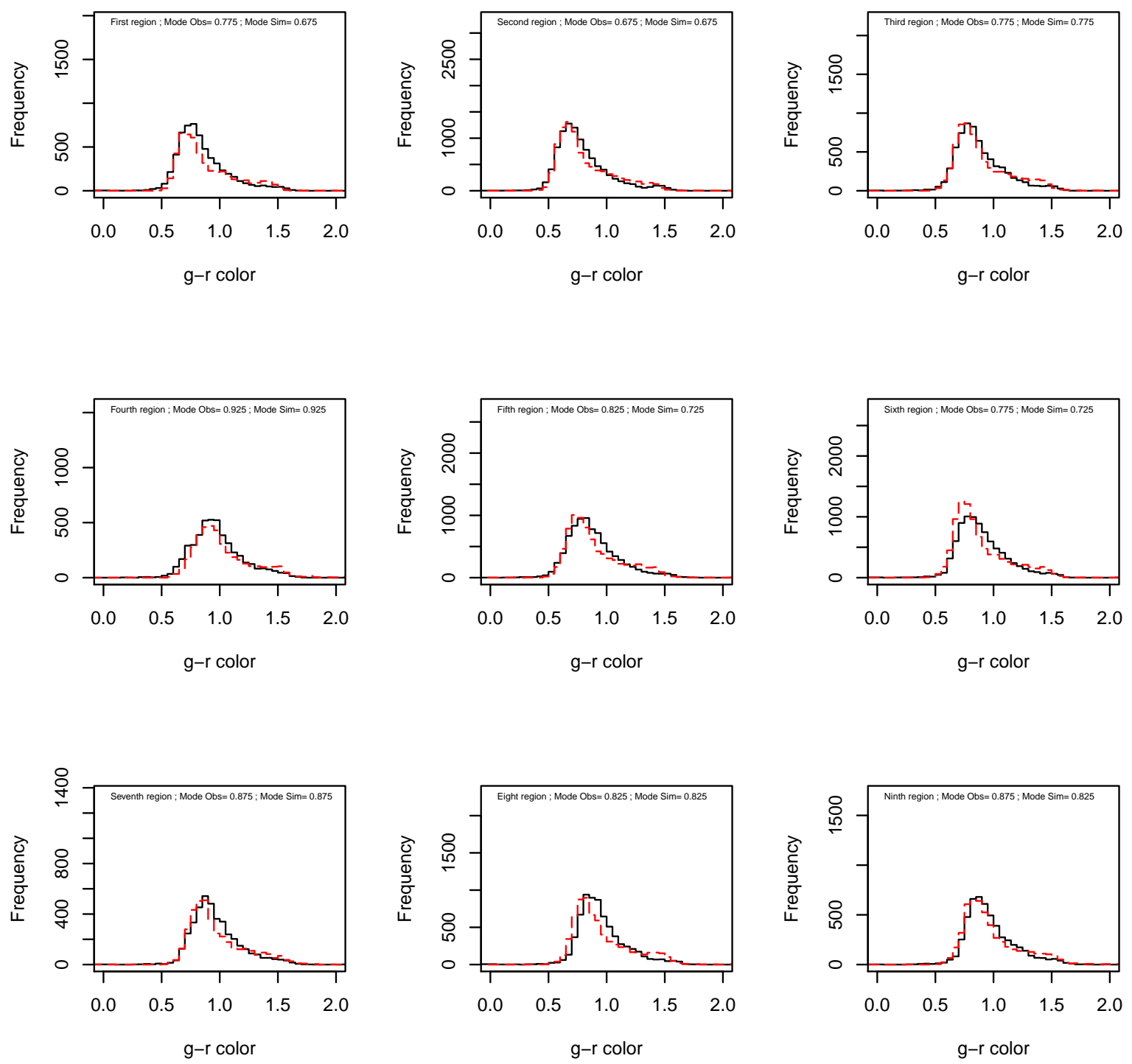

Figure 7.6: Color distributions for each individual region of the field 2537. The black histograms are observations and the red histograms are simulations. 

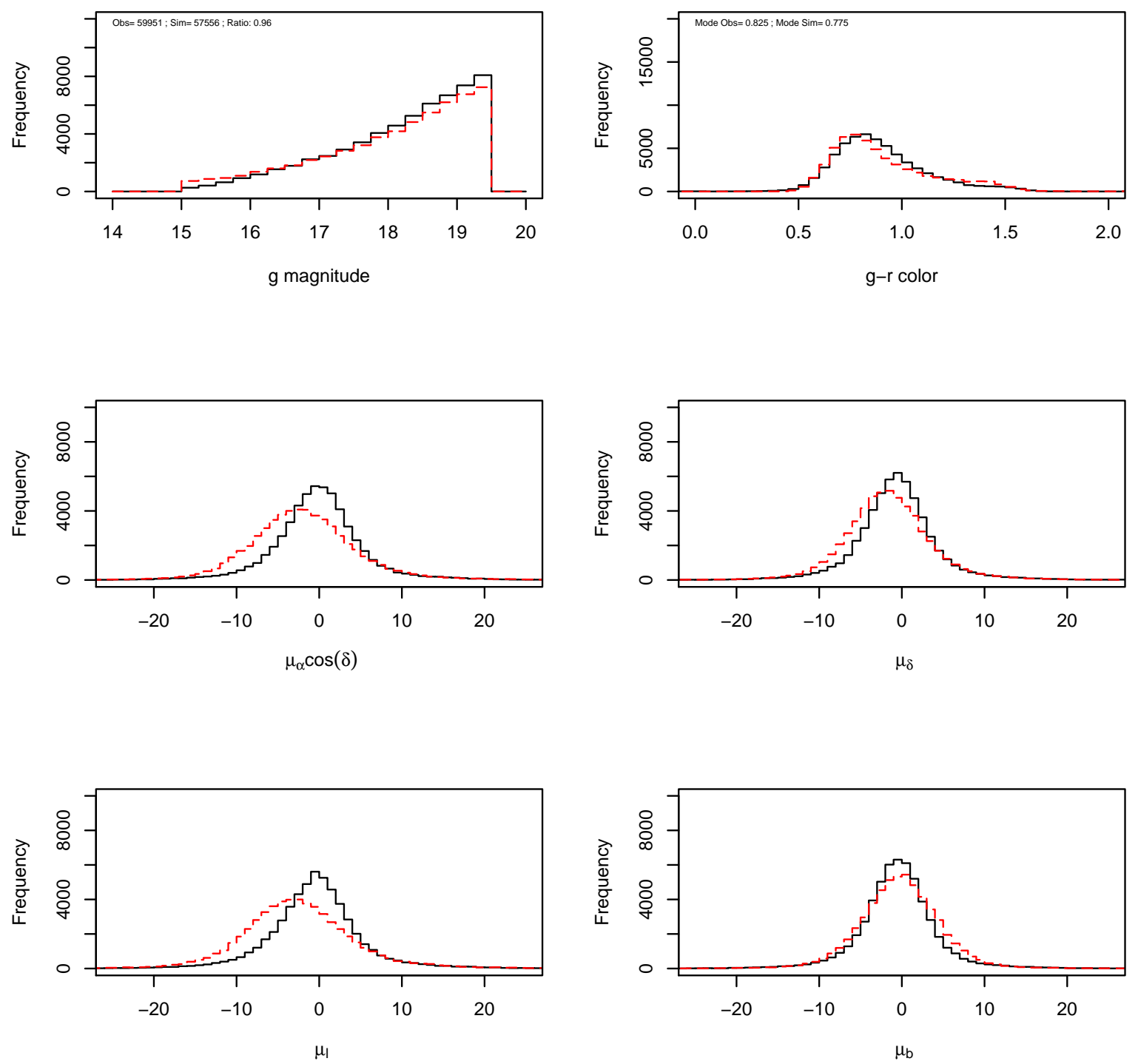

Figure 7.7: Magnitude, color (top panels) and proper motion distributions (middle and bottom panels) for field 2537. Black histograms are observations and the red histograms are simulations. 

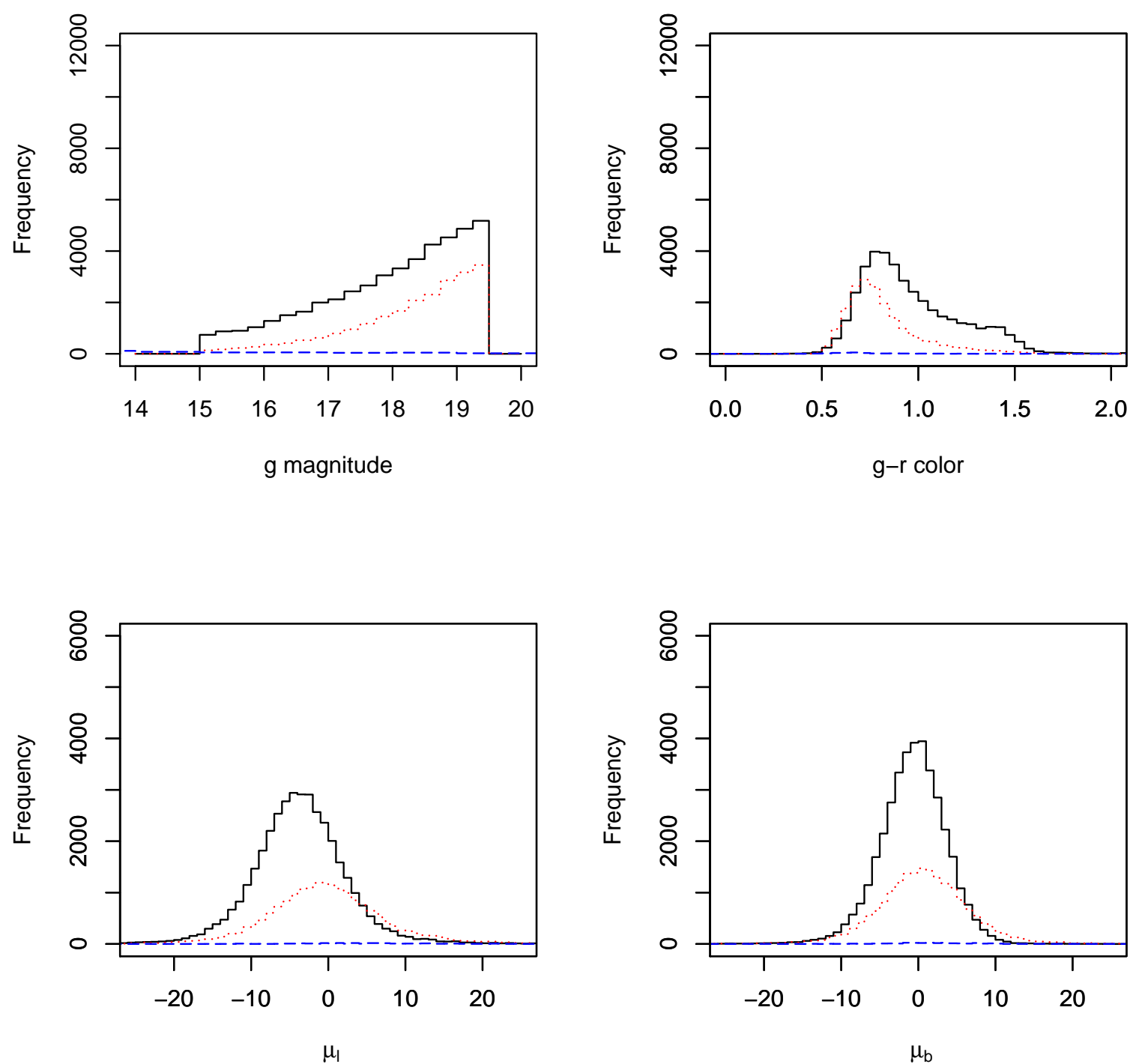

Figure 7.8: Magnitude, color (top panels) and proper motion distributions (bottom panels) separated by populations for field 2537. Thin disc stars: Black solid line; Thick disc stars: Red dotted line; Halo stars: Blue dashed line. 

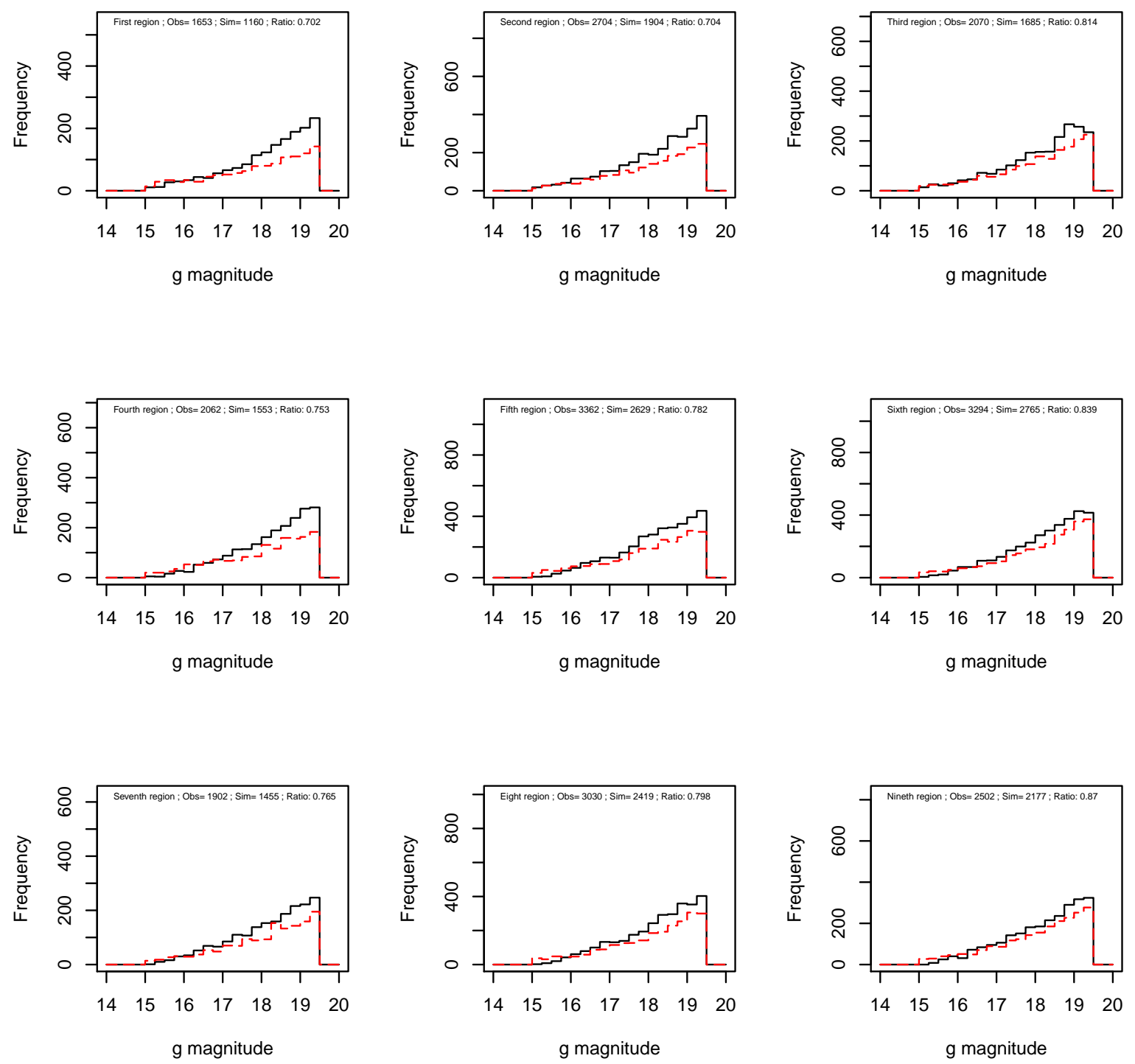

Figure 7.9: Magnitude distribution for each individual region of the field 2537. The black histograms are observations and the red histograms are simulations. 

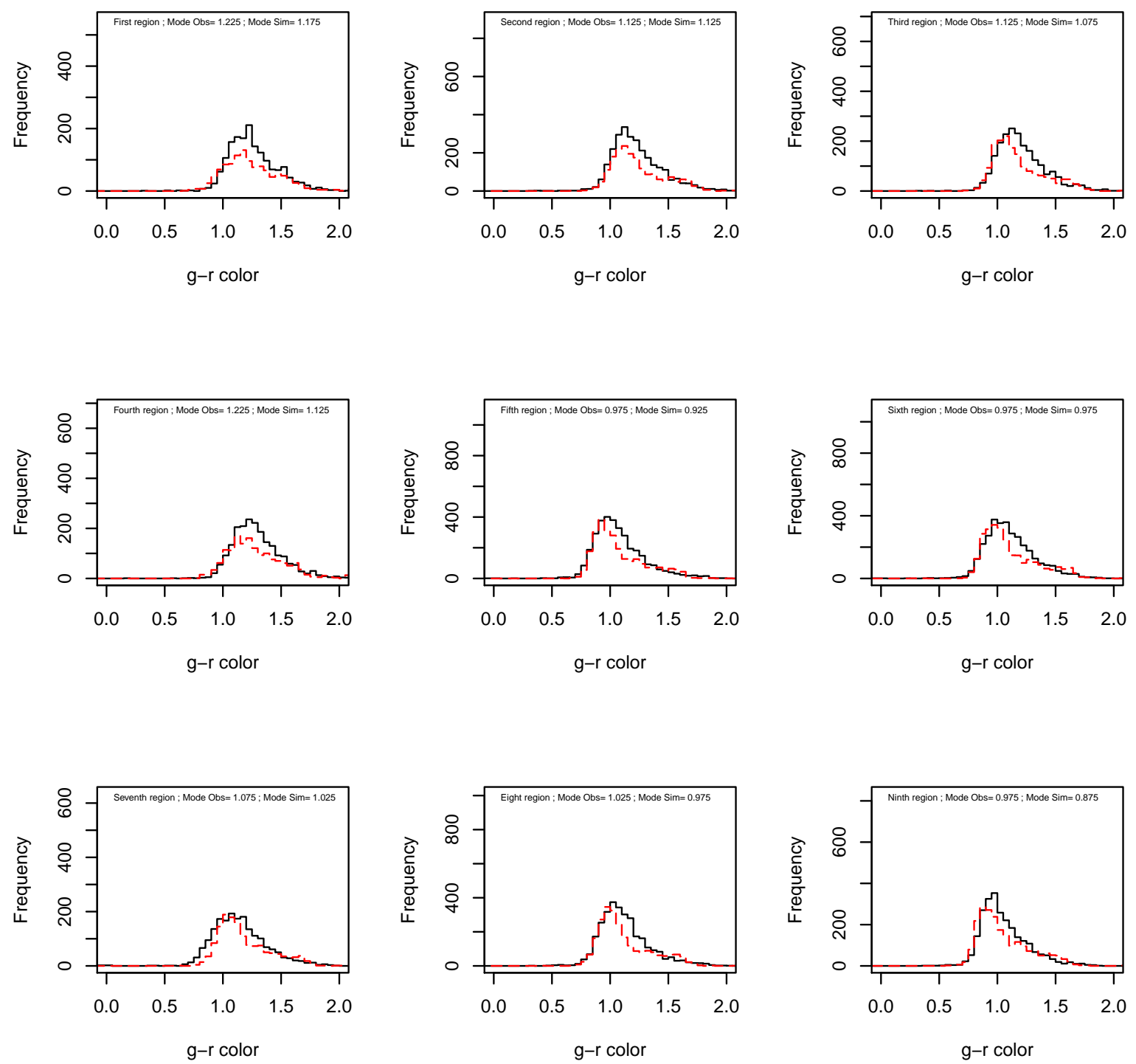

Figure 7.10: Color distributions for each individual region of the field 2537. The black histograms are observations and the red histograms are simulations. 

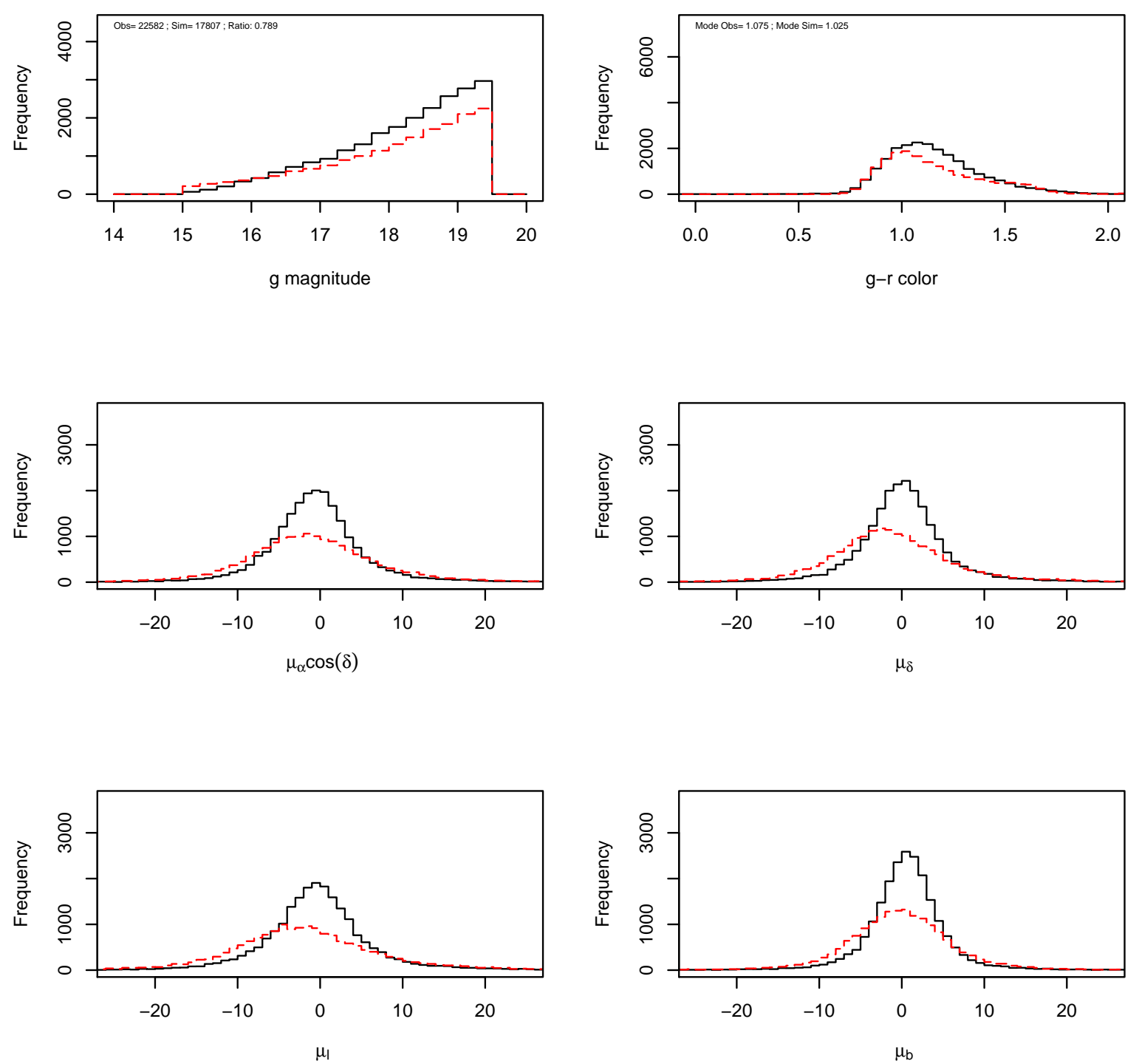

Figure 7.11: Magnitude, color (top panels) and proper motion distributions (middle and bottom panels) for field 2538. Black histograms are observations and the red histograms are simulations. 

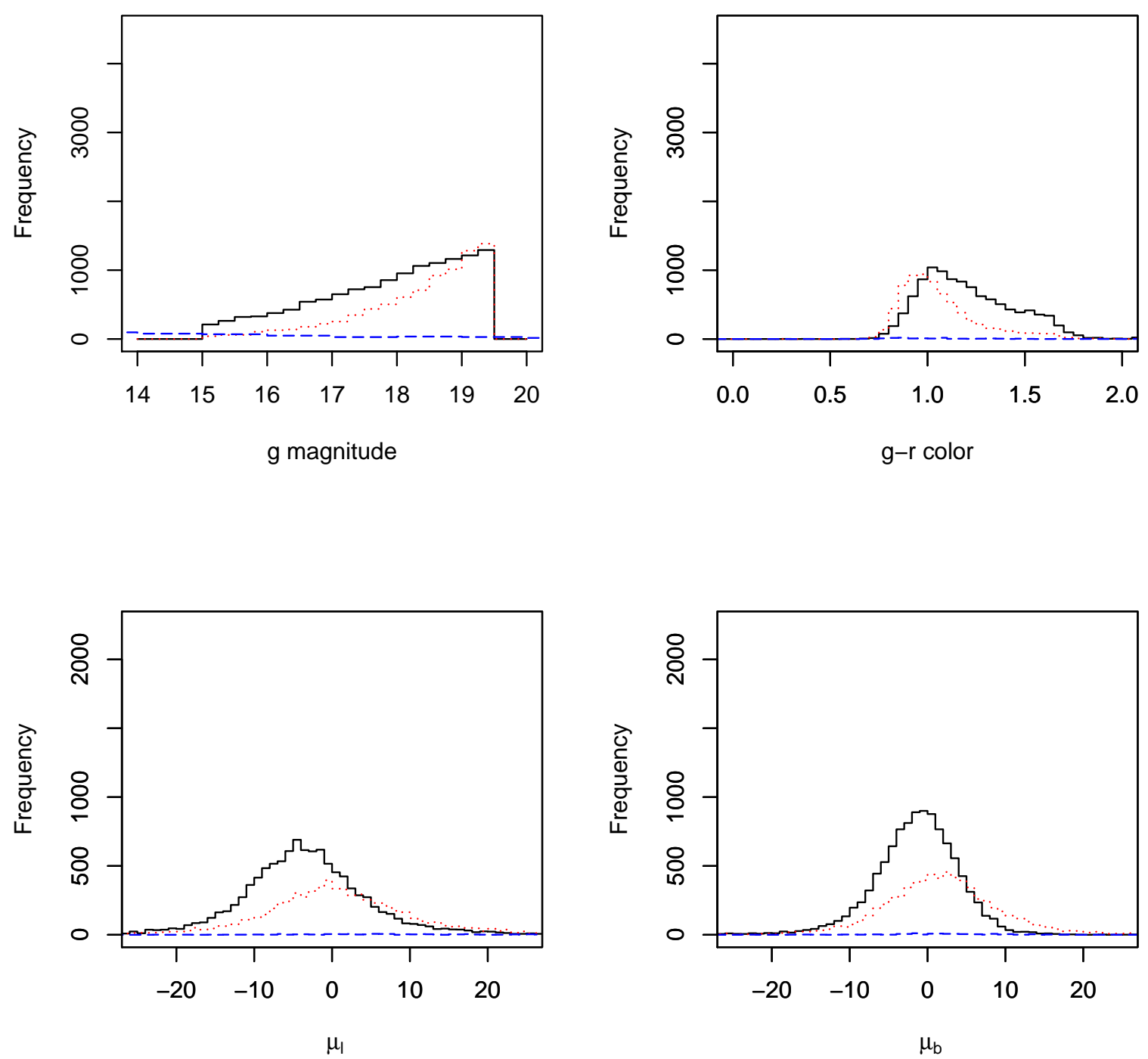

Figure 7.12: Magnitude, color (top panels) and proper motion distributions (bottom panels) separated by populations for field 2538. Thin disc stars: Black solid line; Thick disc stars: Red dotted line; Halo stars: Blue dashed line. 


\subsubsection{Fields 2554, 2555 and 2556}

These three fields are located at the same galactic longitude $\left(l_{2554 / 2555 / 2556}=94^{\circ}\right)$ but at different galactic latitudes $\left(b_{2554}=14^{\circ} \& b_{2555}={ }^{\circ} 8 \& b_{2556}=-8^{\circ}\right)$. The fields 2554 and 2556 suffer similar extinction $\left(A_{V}\right)$ effects. Both have a range of extinction from 0.4 to 1.2. The field 2555 suffers more extinction $\left(A_{V}\right)$ with a range from 0.9 to slightly above 3. Magnitude distributions along with the number of stars in each catalog and their ratio in each region, are shown in figures $7.13,7.14$ and 7.15 for fields 2554, 2555 and 2556 respectively. For field 2554 there is a good agreement between observations and simulations as visible in figure 7.13. The ratio between star counts in each region ranges from 0.90 to 1.0, which shows an approximate constant factor for each region. Figure 7.15 shows, for field 2556, that the quantity of simulated stars is larger than the observed stars by a ratio in the range of 1.1 to 1.2 depending on the region. In figure 7.14 we see that simulations do not reproduce so well the observations for field 2555 . There is a quite low ratio and a large range of values $(0.5$ to 0.9$)$ which demonstrates the complexity of an analysis to this field. Figures 7.16, 7.17 and 7.18 represent the color distributions of each region for each field along with the modes of both distributions. It is visible that for each region, the color distribution is generally reproduced despite the large extinction behaviour in each region for each field. The values of the modes are in agreement even if, for field 2555, the color distributions are less accurate which may indicate that the extinction in this field needed to be fitted with a more complex behaviour. Nevertheless, the simulated color histogram is bluer than the observed ones which means that a stronger extinction will only result in a larger difference in the number of stars. The lack of stars in the positive latitude fields and the existence of too many stars in the simulation for the negative field may be an indication that the warp slope is not correct or that the warp model used is too simple.

Figure 7.19 shows, for the field 2554, from the first two top histograms, the partial good agreement noticed when the individual regions were being analized. The 1 component of the proper motions (bottom panel) show small shift in simulations. The thick disc is a significant component in this field at latitude $14^{\circ}$, as seen in figure 7.20. For the field 2556 results are represented in figure 7.23 and show very similar results but the quantity of stars in the simulations is larger than in observations. Inspection of figure 7.24 shows that the thin disc is the stronger component in the field.

An opposite behaviour can be seen in figure 7.21 for the field 2555. As discussed in figures 7.14 and 7.17, for the individual regions, the star counts in simulations do not reproduce so well the observations. We see a shift (already noticed in other fields like the 2536 in figure 7.21) between simulations and observations in the 1 component of the proper motion that is not present in the $\mathrm{b}$ component and the dispersion in simulations is larger. The shifts in the 1 component of the proper motion, observed for the other two fields, may be due to the asymmetric drift which values may need to be updated. A comparison with the other two fields shows a huge difference in star counts that is probably due to the high extinction present in the field probably due to the clumpy extinction pattern near the sun as shown in the first two panels of figure 2.12. The huge difference between star counts in the observations and simulations, that is not present in the other two fields, may be an indication that the warp model has to be improved or that there is a structure present at this direction even if the metallicity distributions do not show a 

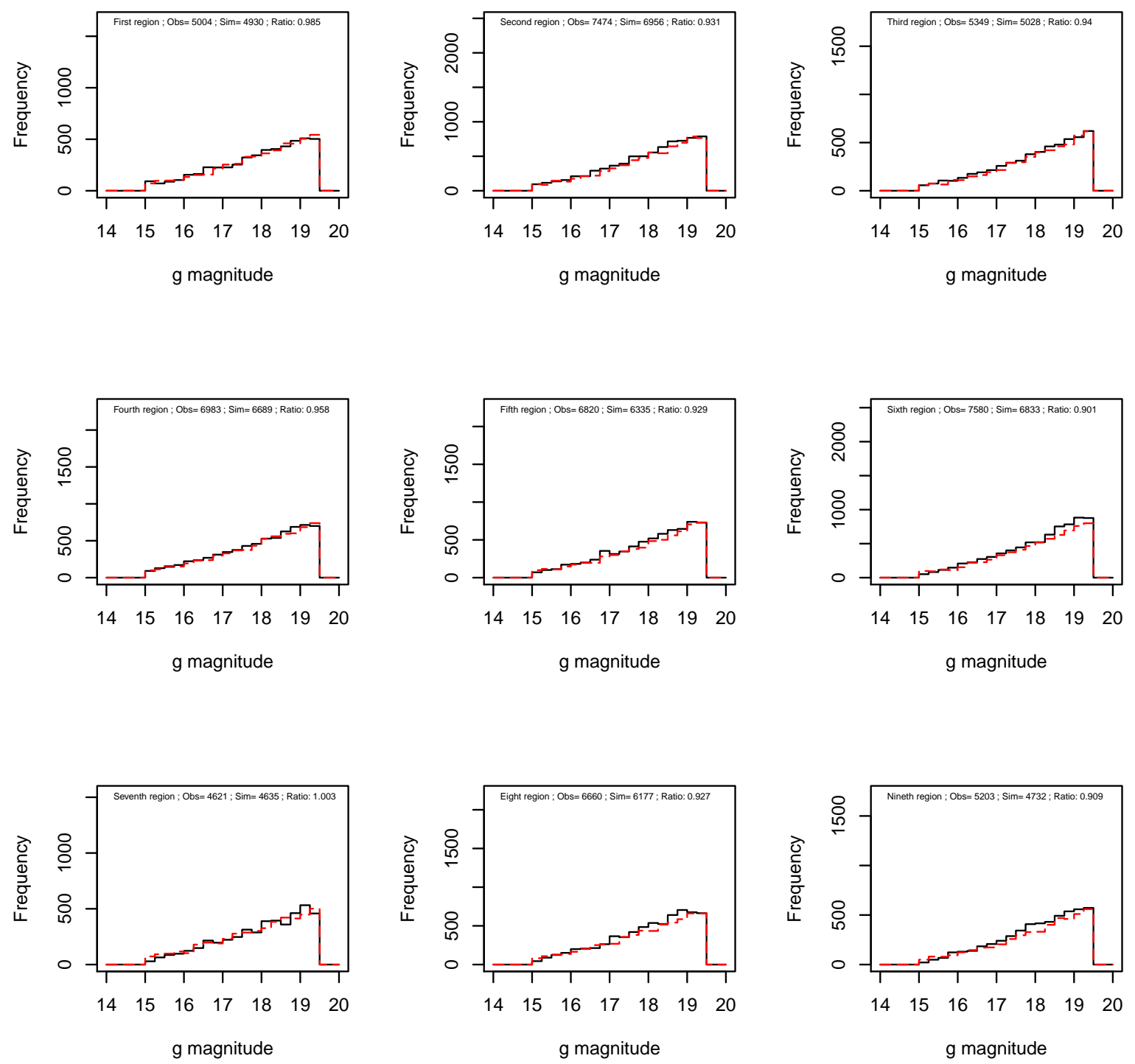

Figure 7.13: Magnitude distribution for each individual region of the field 2554. The black histograms are observations and the red histograms are simulations. 

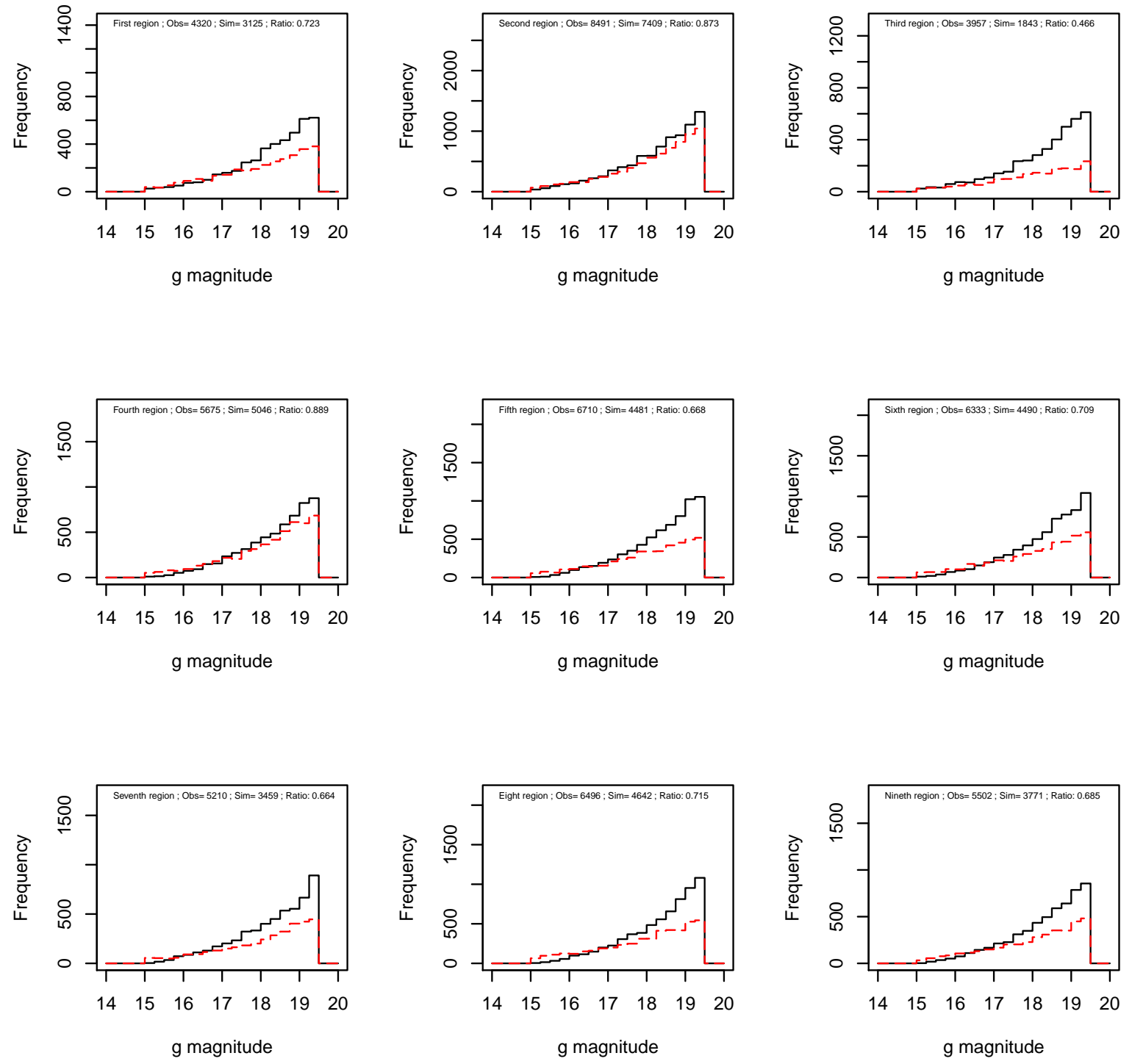

Figure 7.14: Magnitude distribution for each individual region of the field 2555. The black histograms are observations and the red histograms are simulations. 

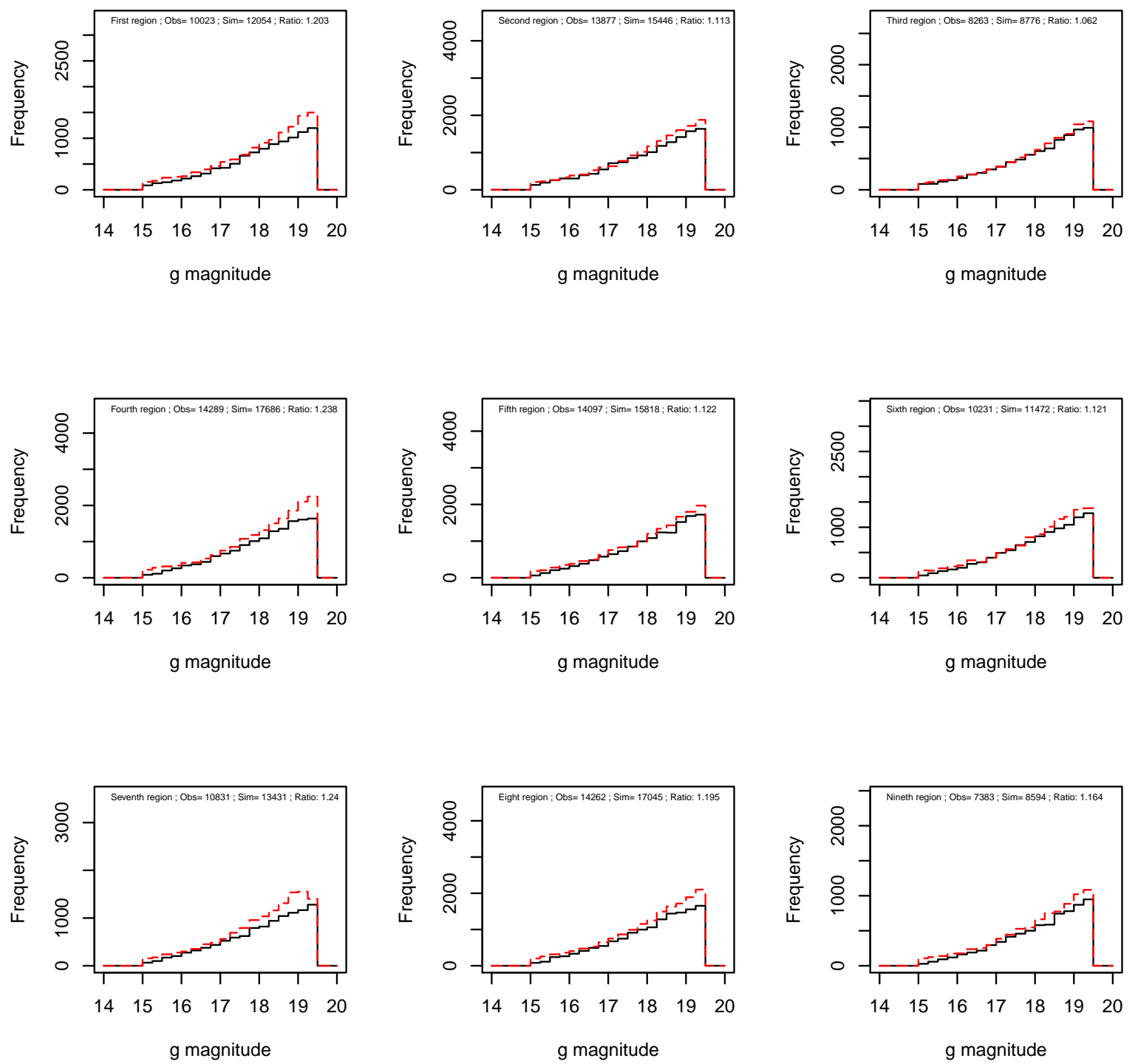

Figure 7.15: Magnitude distribution for each individual region of the field 2556. The black histograms are observations and the red histograms are simulations. 

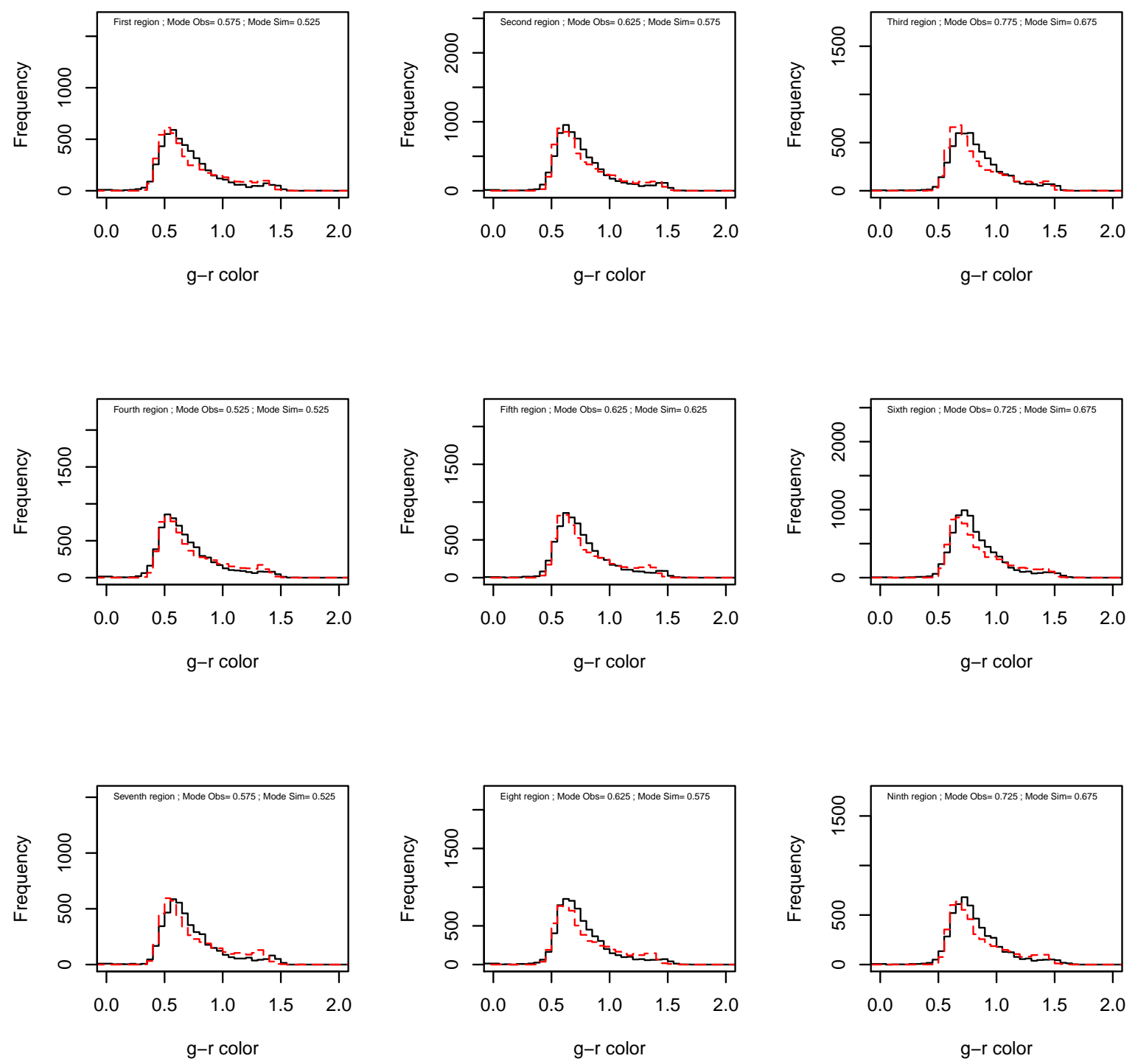

Figure 7.16: Color distribution for each individual region of the field 2554. The black histograms are observations and the red histograms are simulations. 

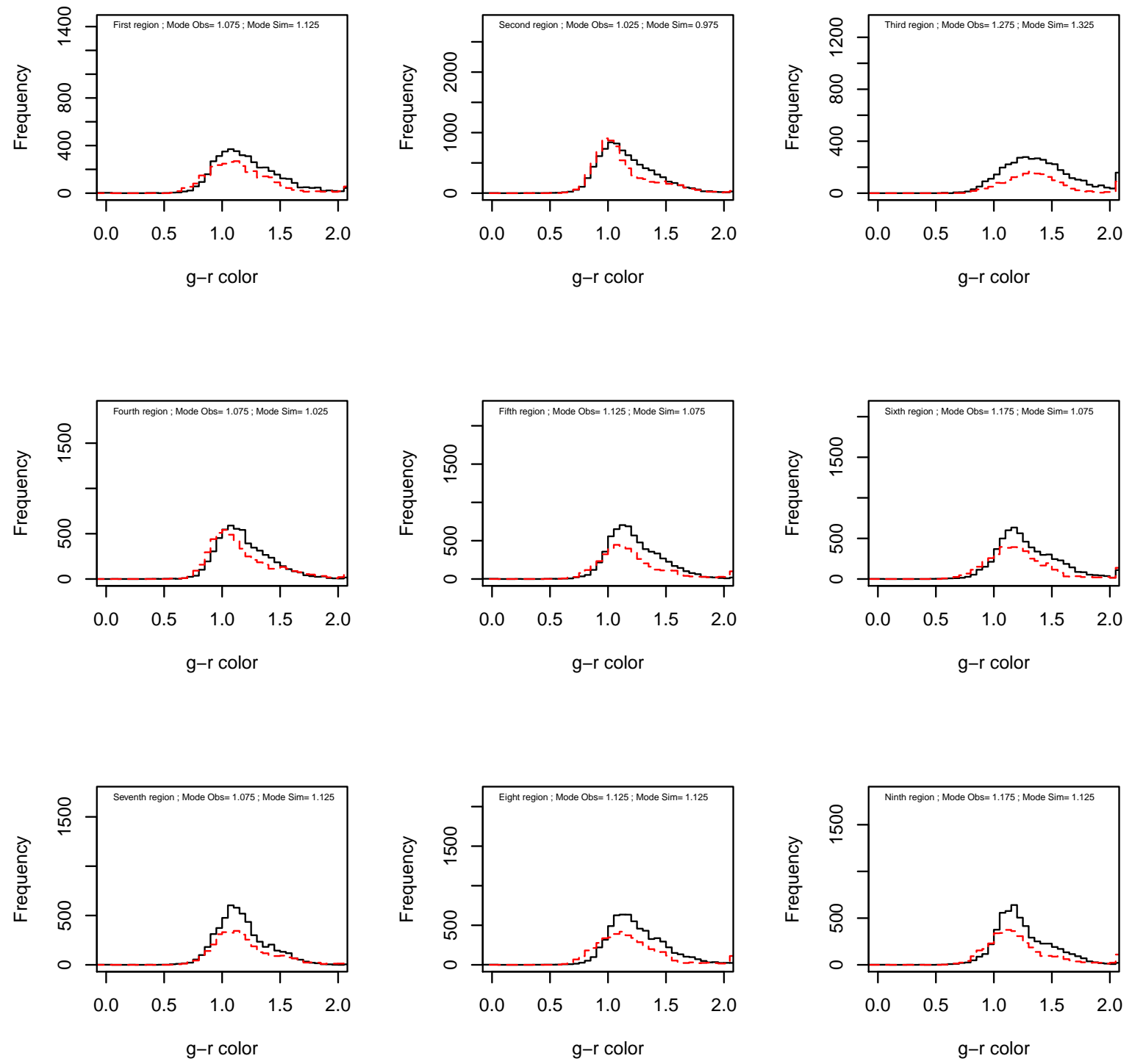

Figure 7.17: Color distribution for each individual region of the field 2555. The black histograms are observations and the red histograms are simulations. 

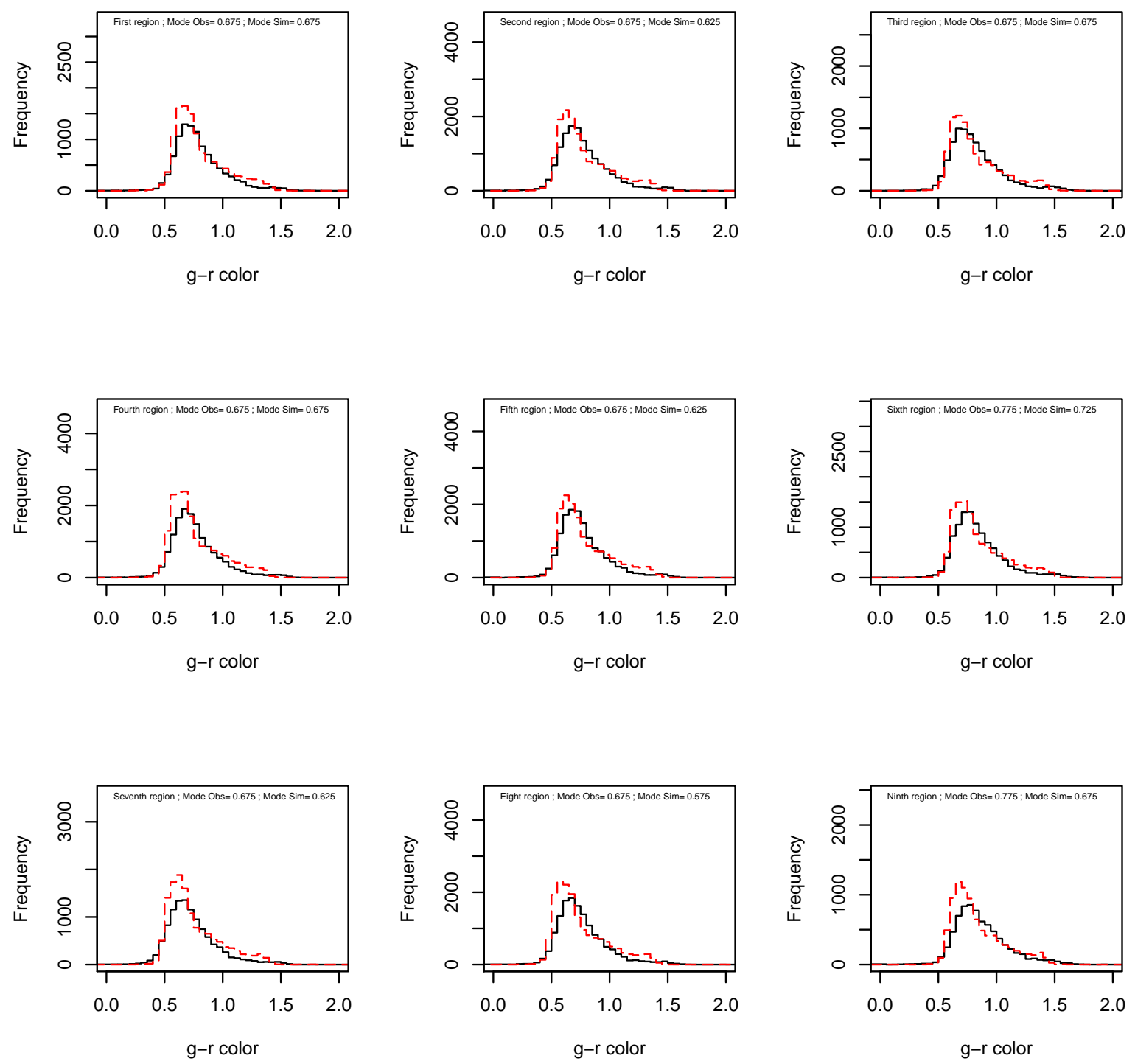

Figure 7.18: Color distribution for each individual region of the field 2556. The black histograms are observations and the red histograms are simulations. 
disagreement. 

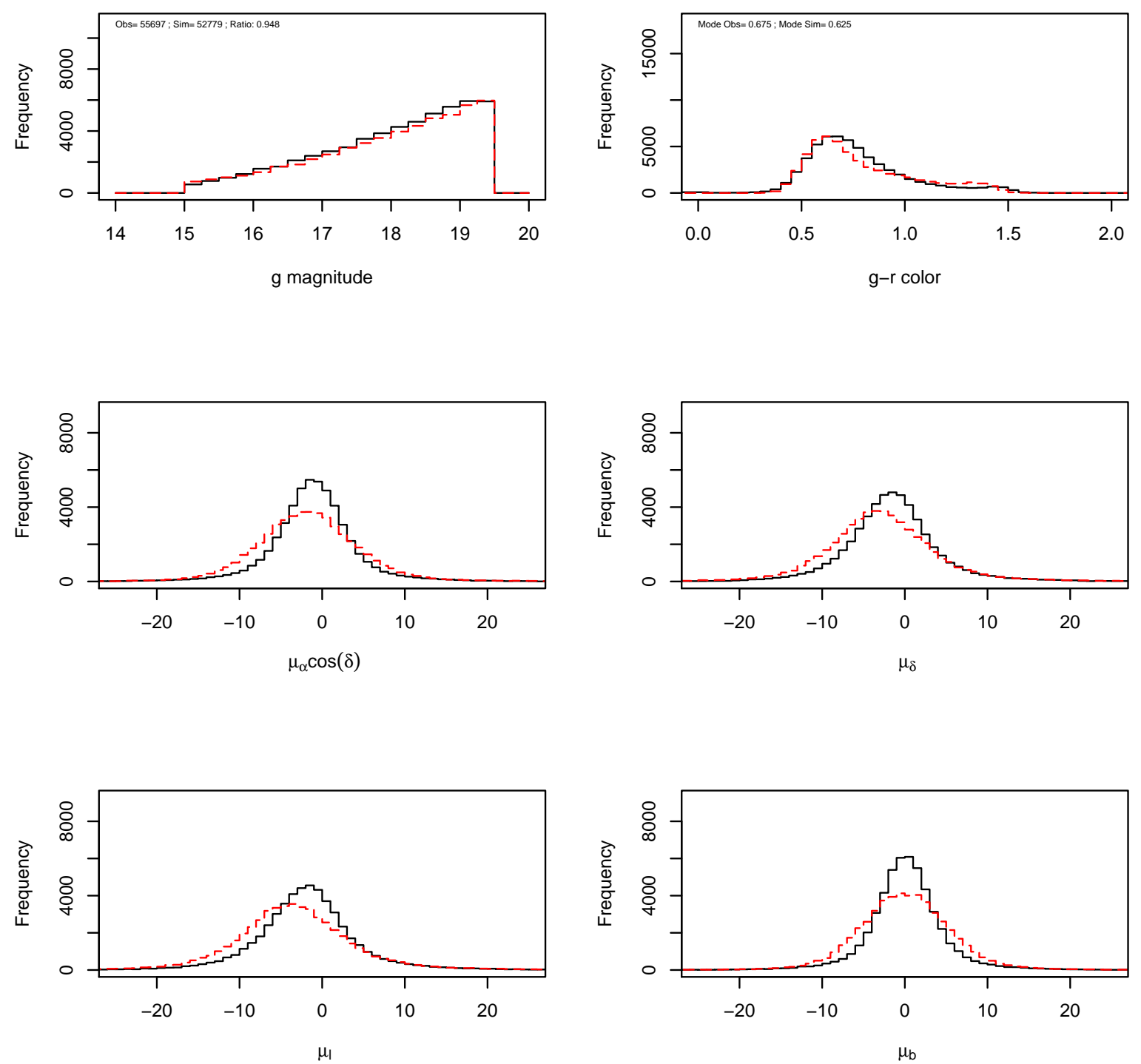

Figure 7.19: Magnitude, color (top panels) and proper motion distributions (middle and bottom panels) for field 2554. Black histograms are observations and the red histograms are simulations. 

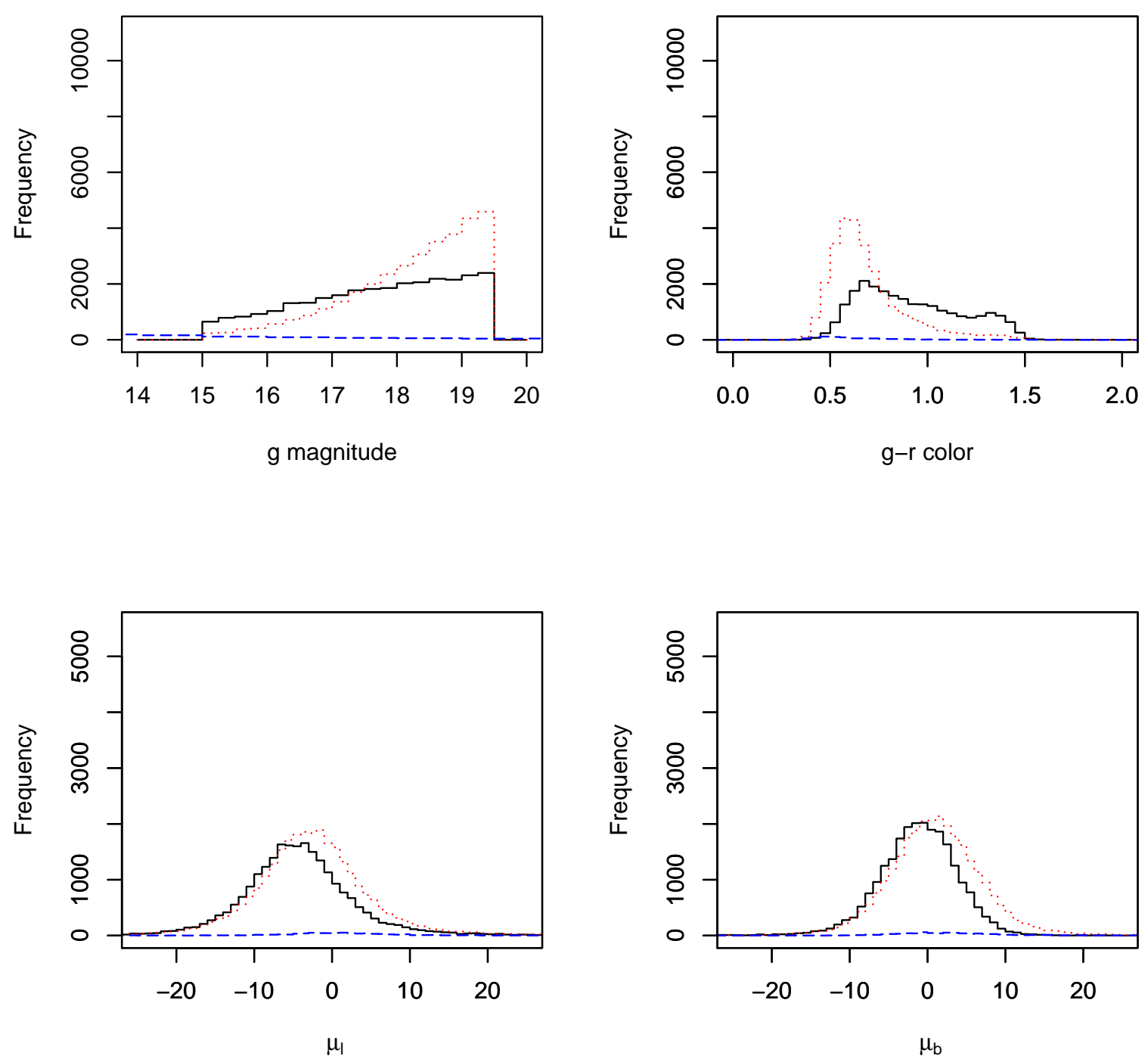

Figure 7.20: Magnitude, color (top panels) and proper motion distributions (bottom panels) separated by populations for field 2554. Thin disc stars: Black solid line; Thick disc stars: Red dotted line; Halo stars: Blue dashed line. 

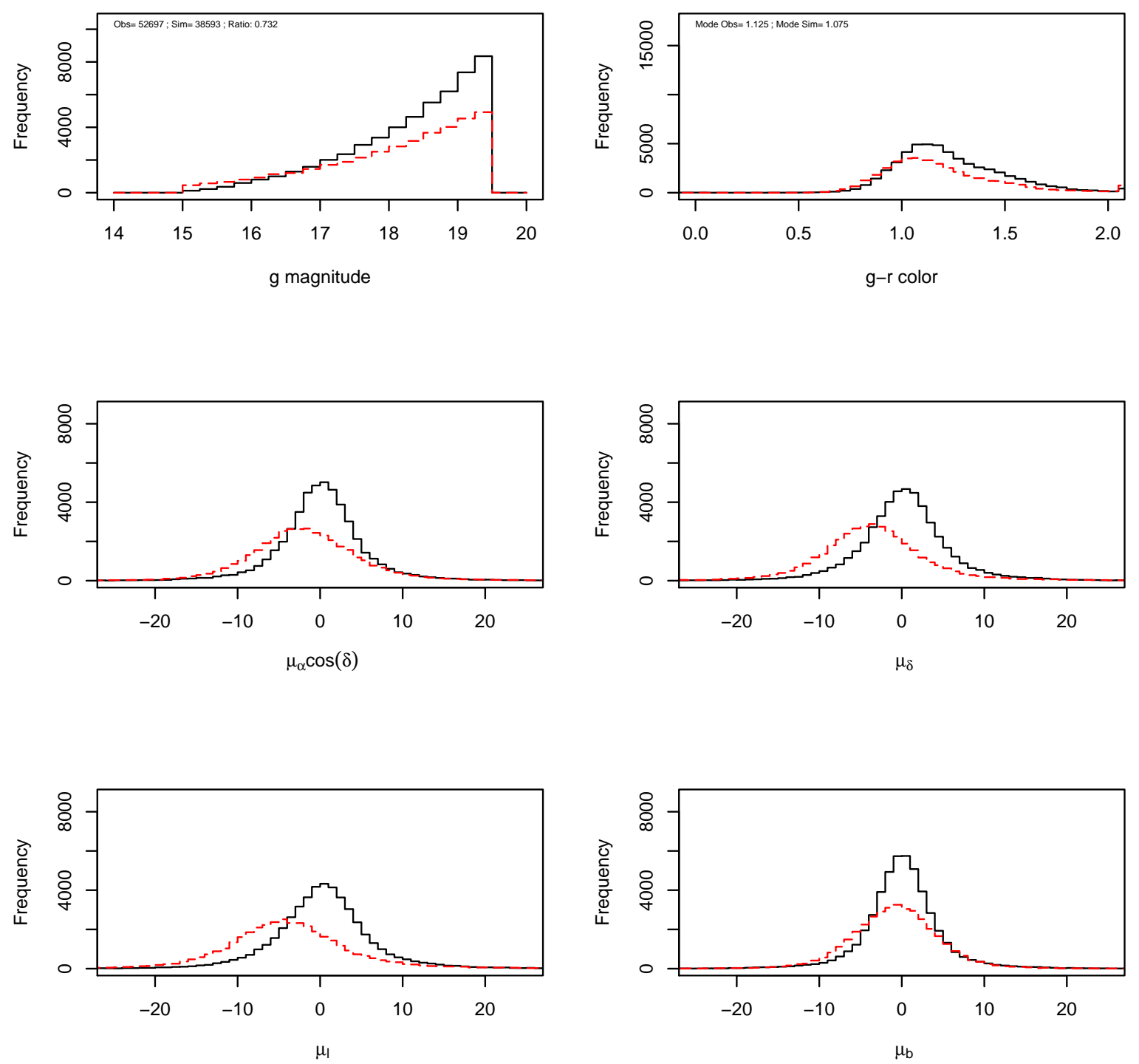

Figure 7.21: Magnitude, color (top panels) and proper motion distributions (middle and bottom panels) for field 2555. Black histograms are observations and the red histograms are simulations. 

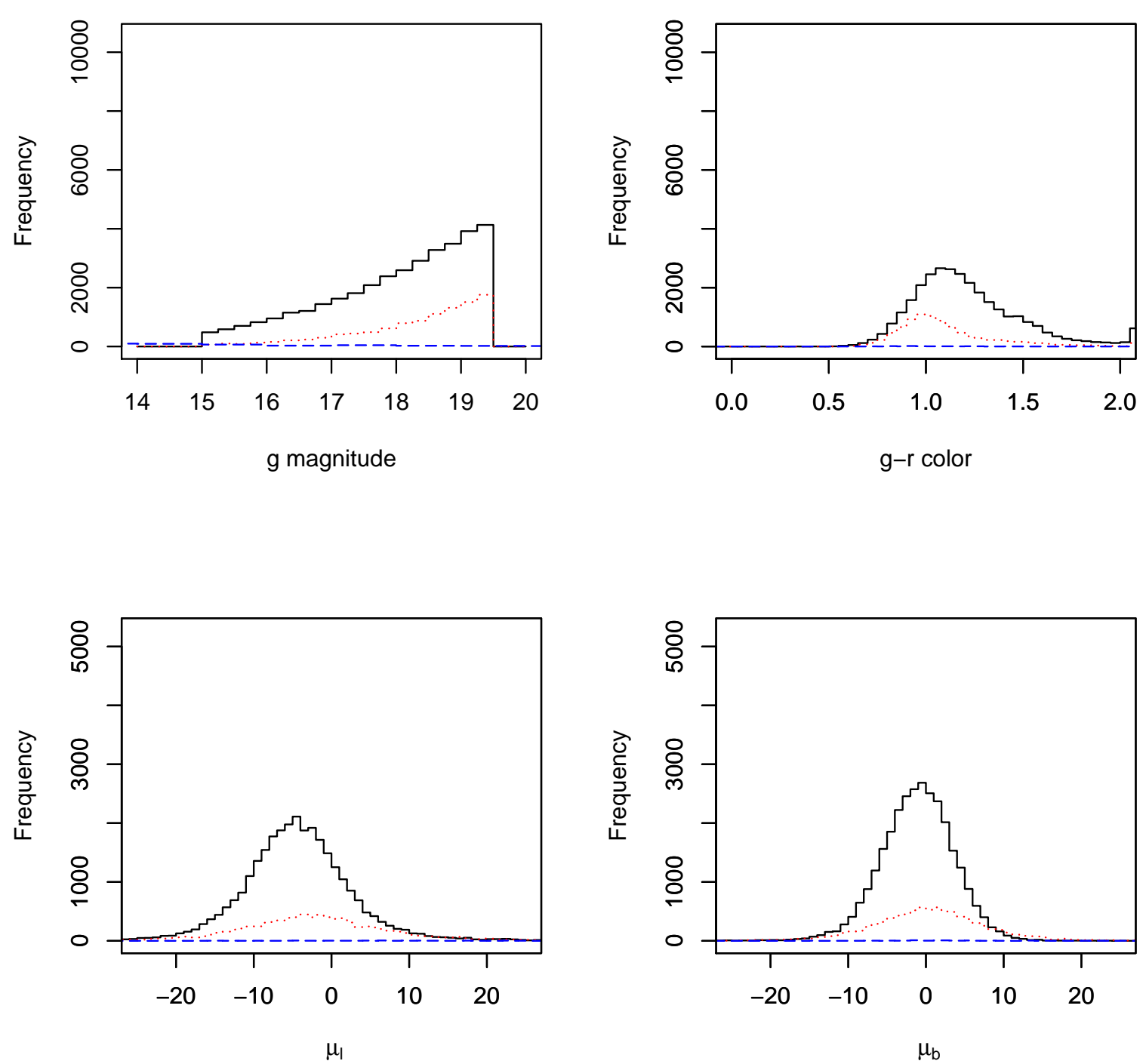

Figure 7.22: Magnitude, color (top panels) and proper motion distributions (bottom panels) separated by populations for field 2555. Thin disc stars: Black solid line; Thick disc stars: Red dotted line; Halo stars: Blue dashed line. 

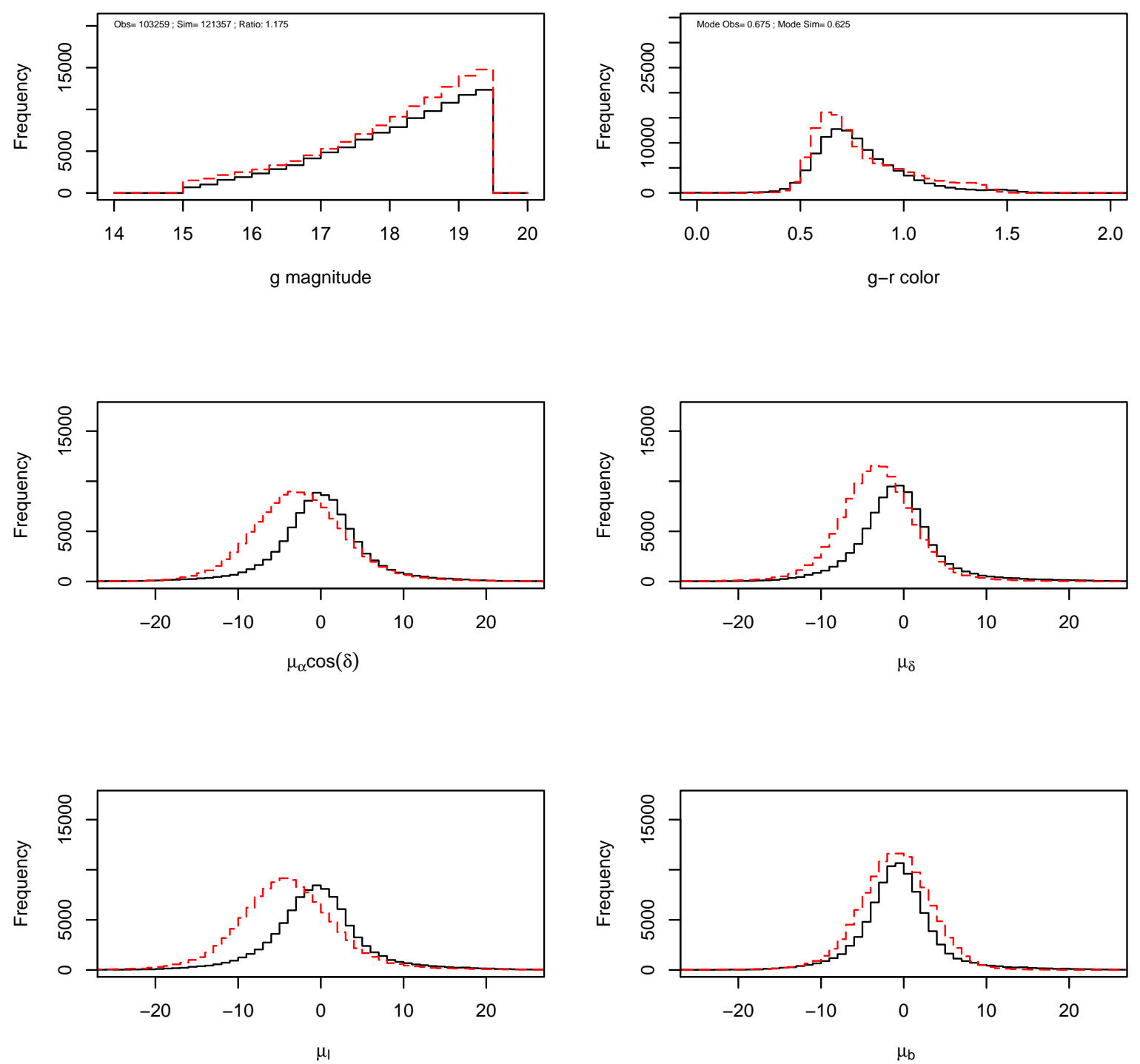

Figure 7.23: Magnitude, color (top panels) and proper motion distributions (middle and bottom panels) for field 2556. Black histograms are observations and the red histograms are simulations. 

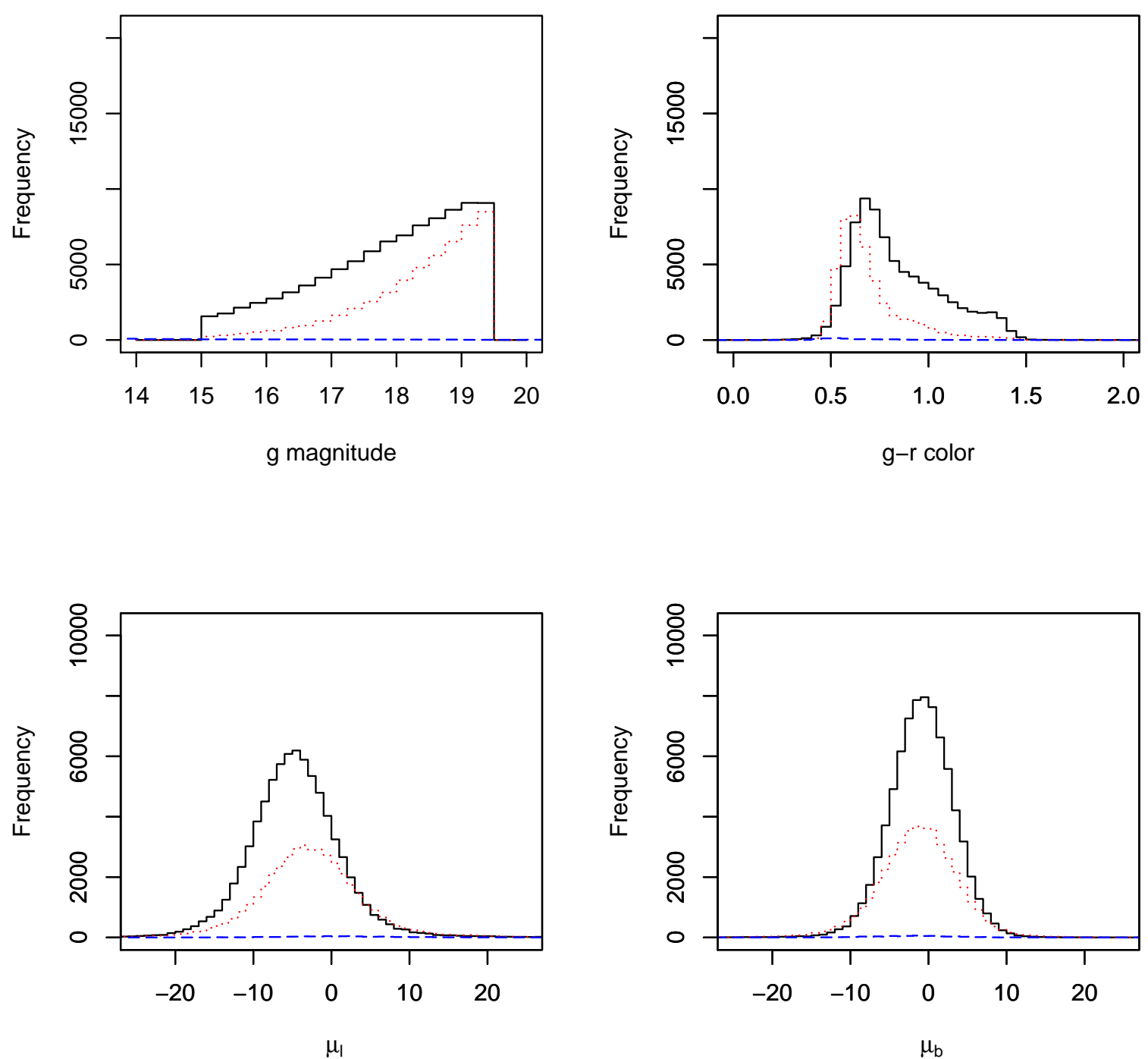

Figure 7.24: Magnitude, color (top panels) and proper motion distributions (bottom panels) separated by populations for field 2556. Thin disc stars: Black solid line; Thick disc stars: Red dotted line; Halo stars: Blue dashed line. 


\subsubsection{Fields 2668, 2678 and 2681}

These fields are located at the anticenter directions and they suffer moderately from extinction $\left(\mathrm{A}_{V}\right)$. Fields $2668\left(l_{2668}=187^{\circ}, b_{2668}=-12^{\circ}\right)$ and $2678\left(l_{2678}=187^{\circ}, b_{2678}=8^{\circ}\right)$ have a range of extinction from 0.5 to 1.0 whereas field $2681\left(l_{2681}=178^{\circ}, b_{2681}=-15^{\circ}\right)$ has extinction values ranging from 0.5 to 1.5 . Figures 7.25 to 7.27 show the magnitude distribution along with number of stars in each catalog and the ratio from each region of each field. Figures 7.28 to 7.30 present the color distribution along with the modes from each region of each field. The three fields present similar characteristics. The magnitude and color simulated distributions are well in agreement with observations for each region in each field. The overall distributions, for each field, presented in figures 7.31, 7.33 and 7.35 show a good agreement between observations and simulations. The proper motions in galactic coordinates are well in agreement even if there is a relatively small shift. Figures 7.32, 7.34 and 7.36 show the larger contribution of the thin disc relatively to the thick disc due to the shorter thick disc scale length assumed in the model. 

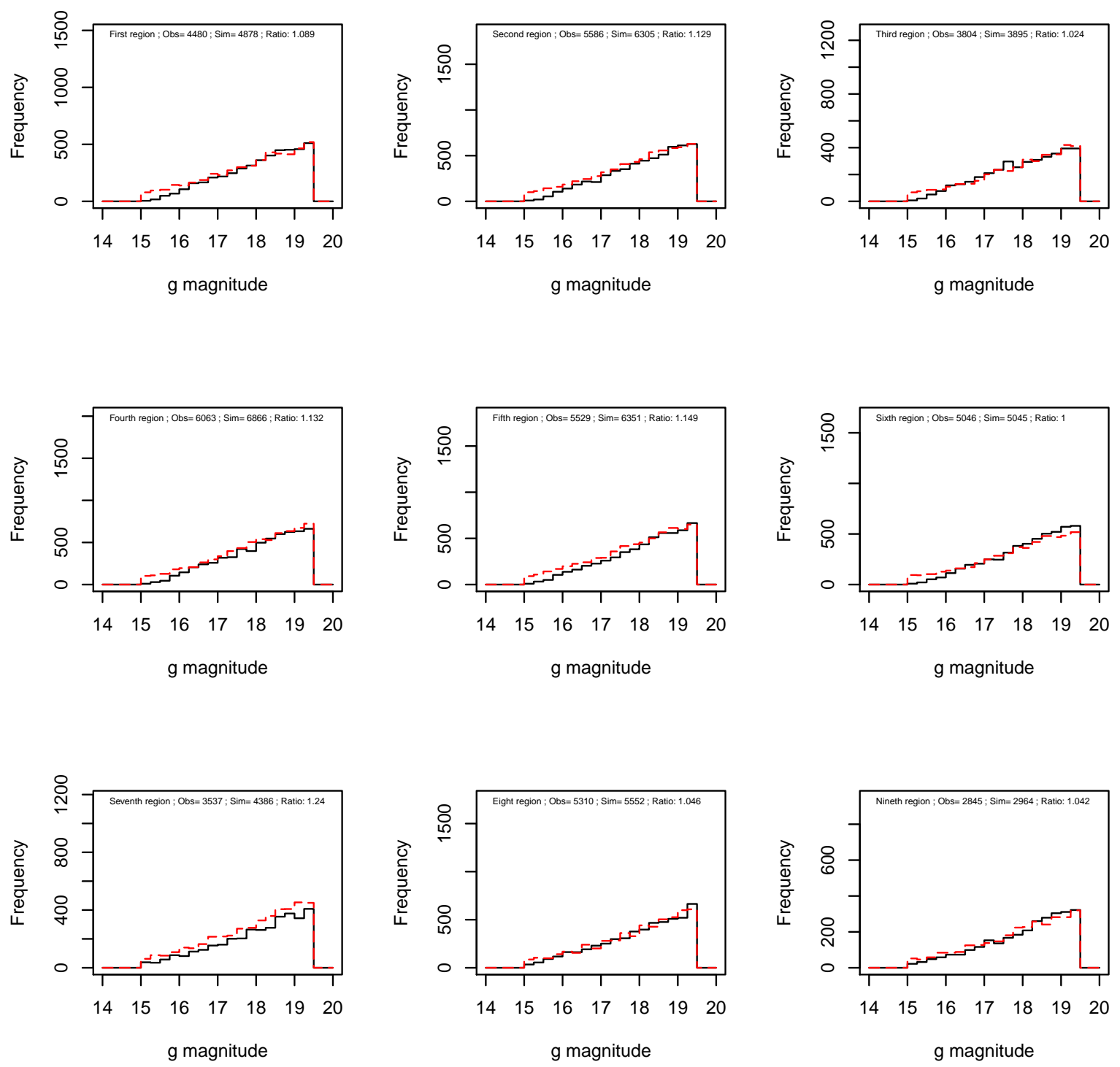

Figure 7.25: Magnitude distribution for each individual region of the field 2668. The black histograms are observations and the red histograms are simulations. 

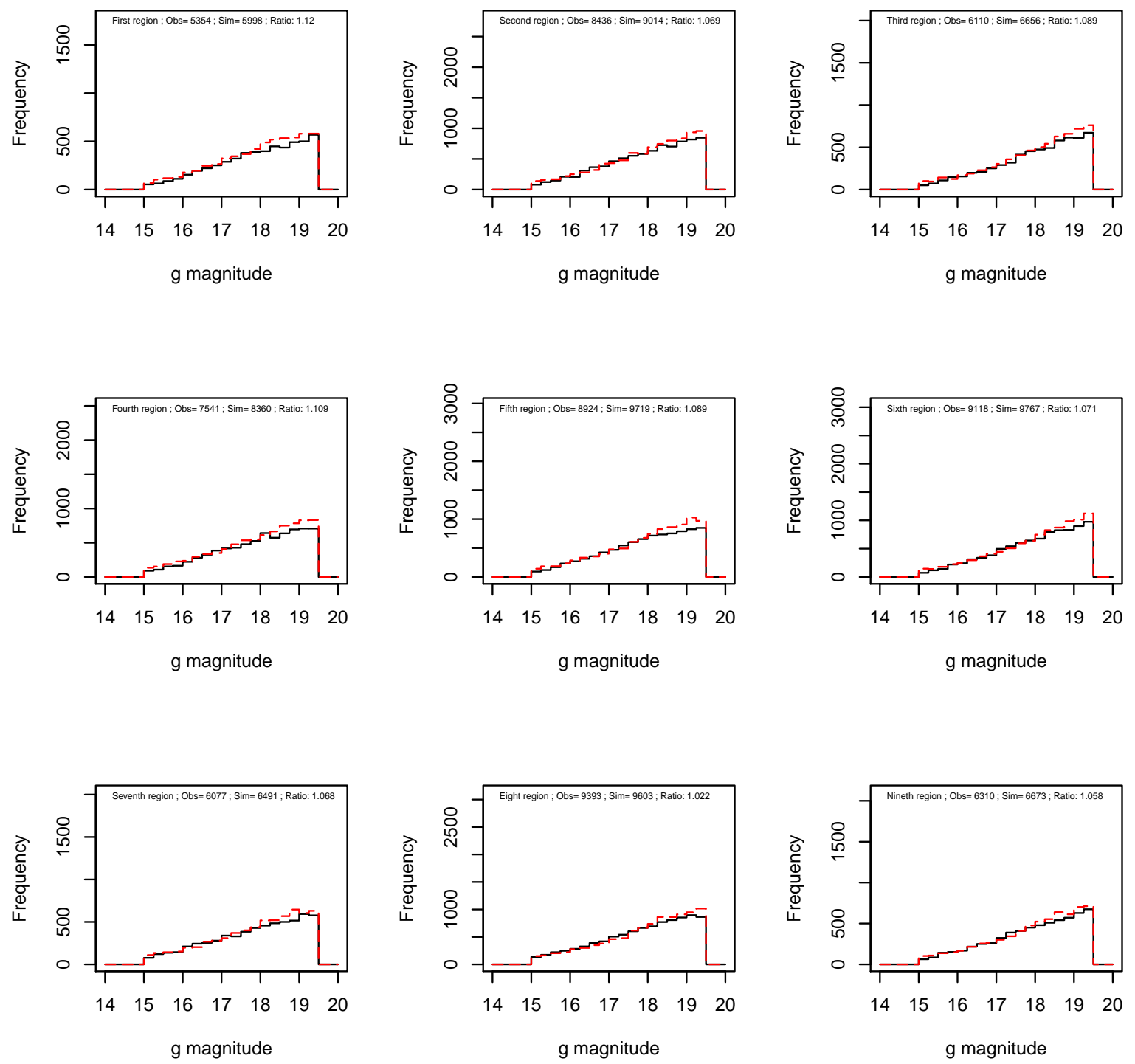

Figure 7.26: Magnitude distribution for each individual region of the field 2678. The black histograms are observations and the red histograms are simulations. 

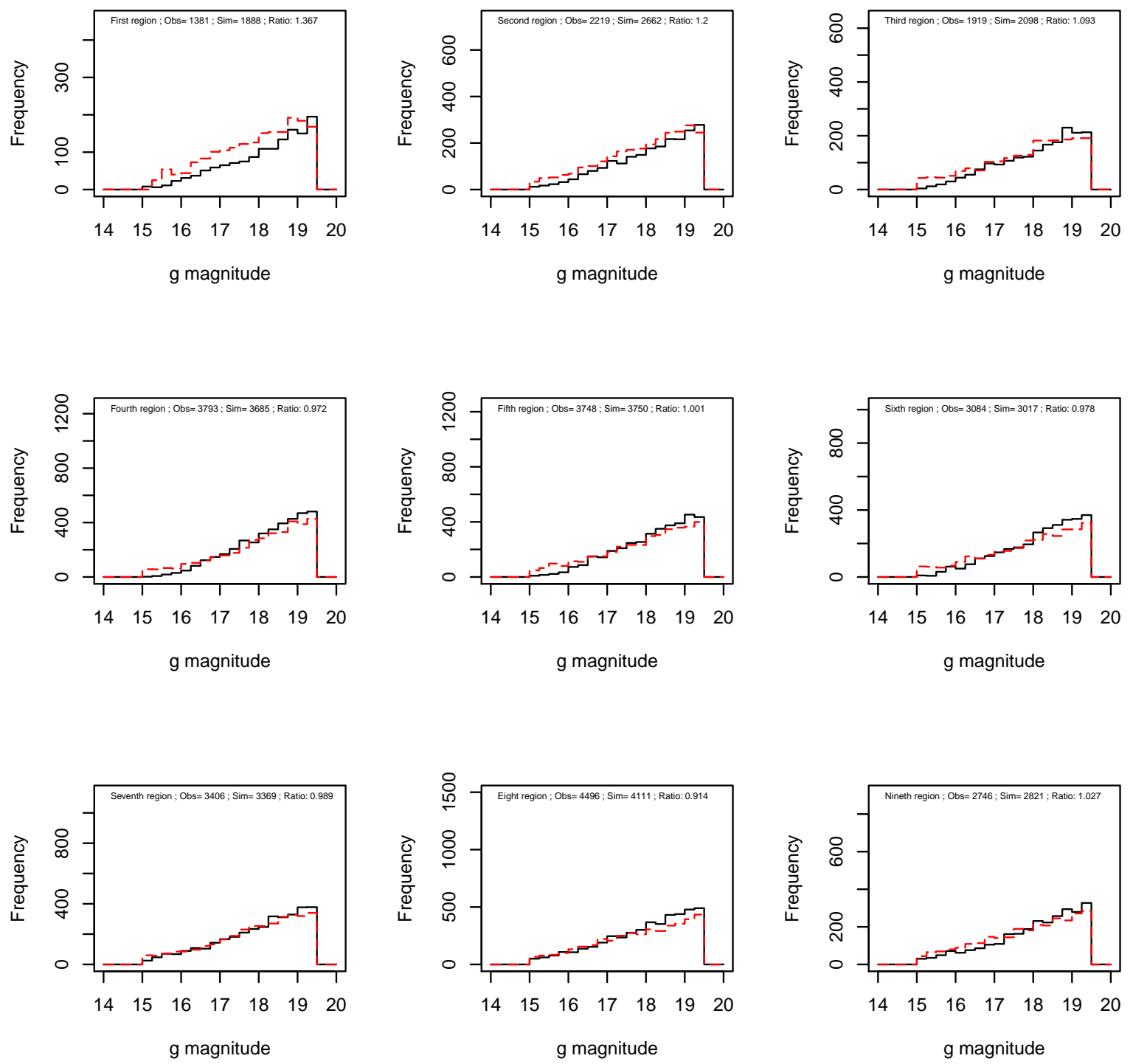

Figure 7.27: Magnitude distribution for each individual region of the field 2681. The black histograms are observations and the red histograms are simulations. 

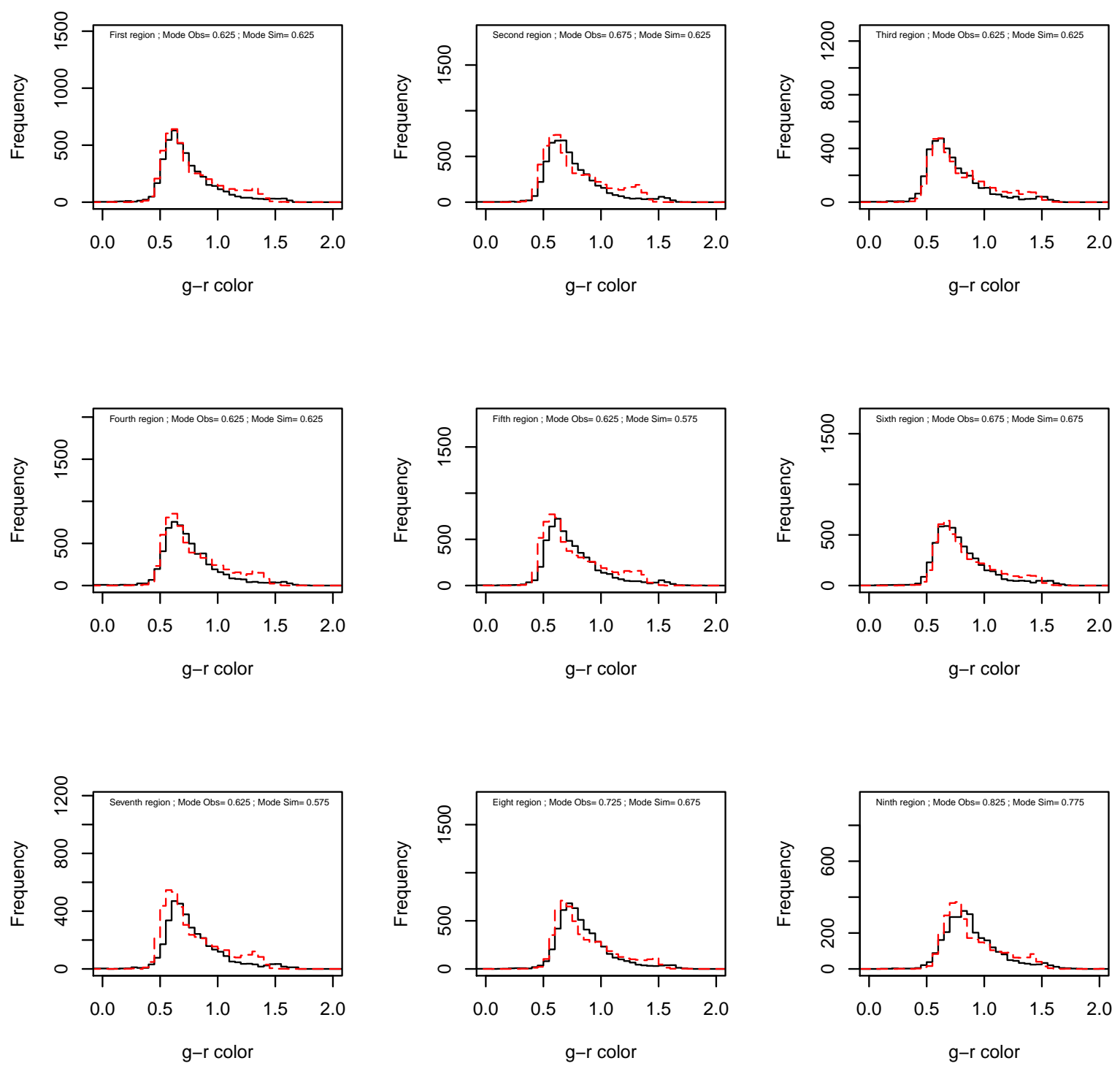

Figure 7.28: Color distribution for each individual region of the field 2668. The black histograms are observations and the red histograms are simulations. 

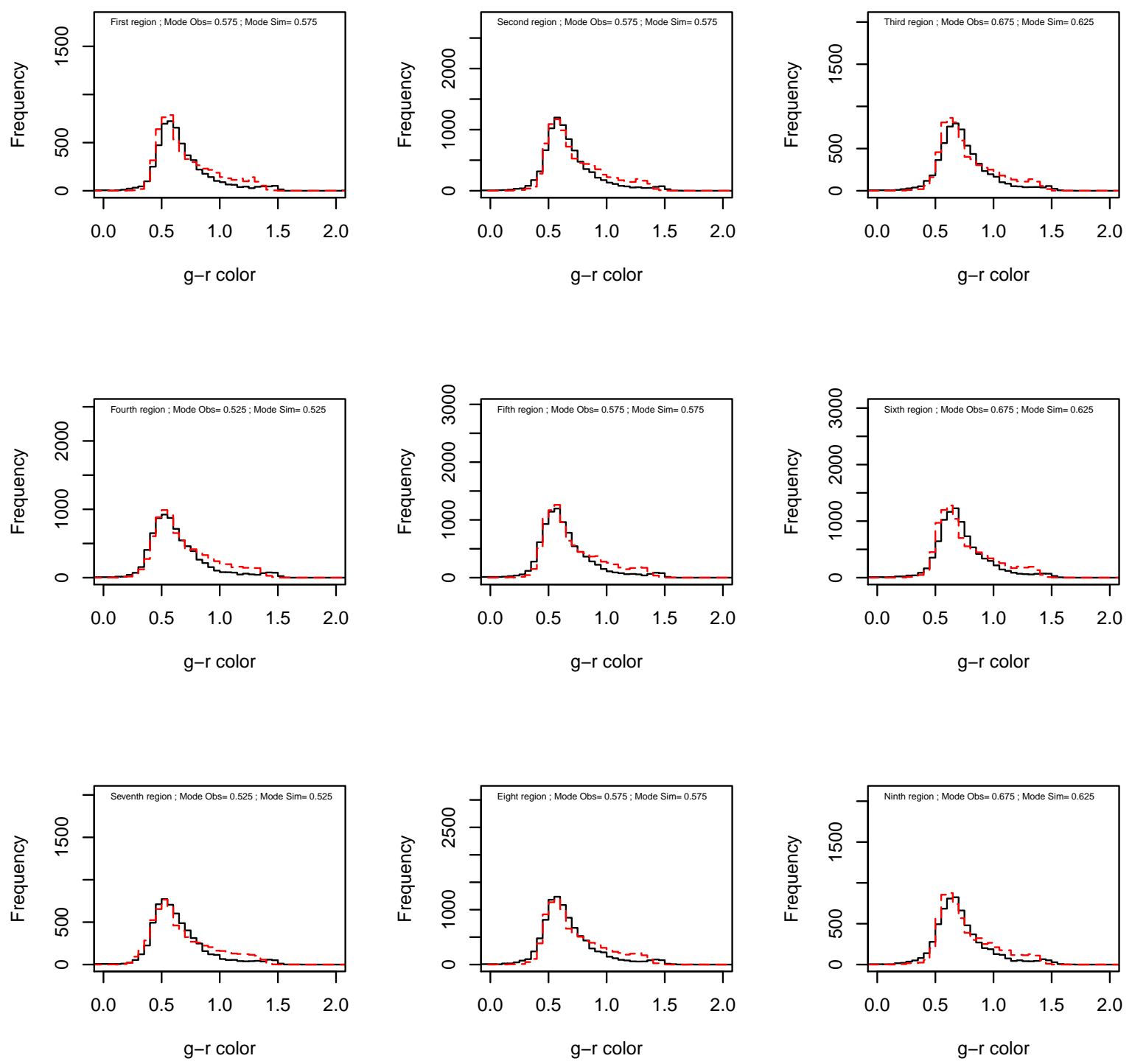

Figure 7.29: Color distribution for each individual region of the field 2678. The black histograms are observations and the red histograms are simulations. 

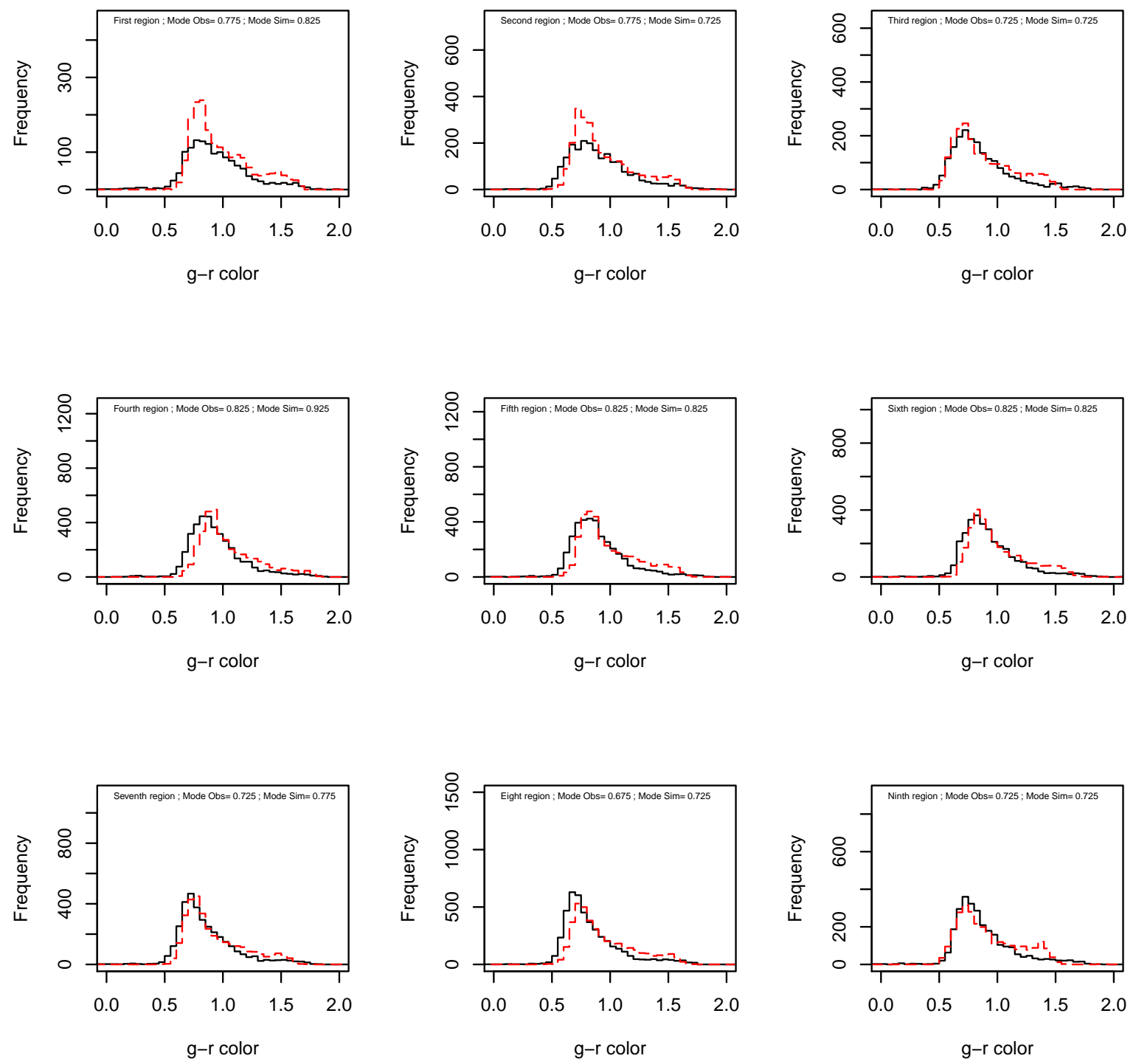

Figure 7.30: Color distribution for each individual region of the field 2681. The black histograms are observations and the red histograms are simulations. 

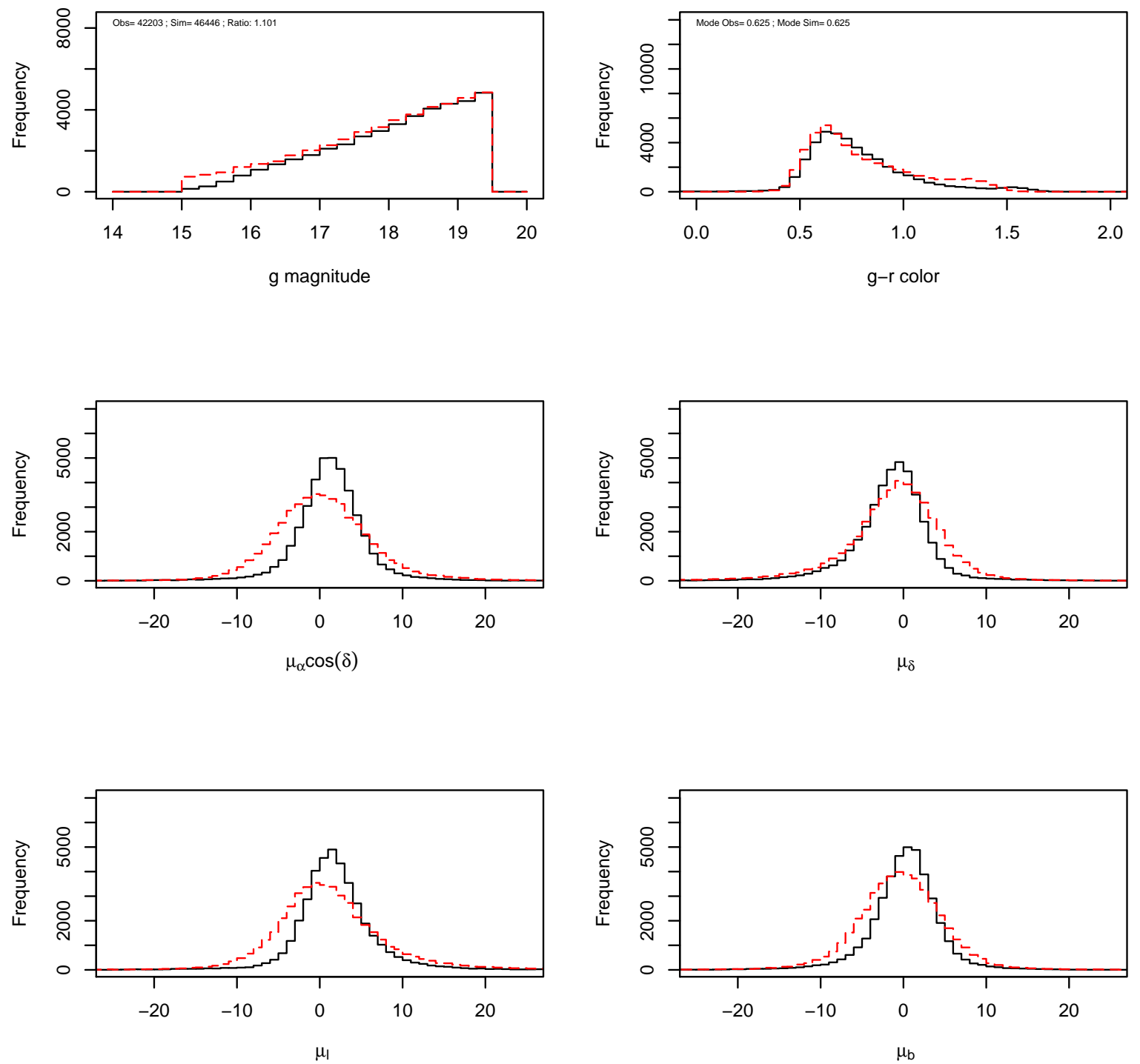

Figure 7.31: Magnitude, color (top panels) and proper motion distributions (middle and bottom panels) for field 2668. Black histograms are observations and the red histograms are simulations. 

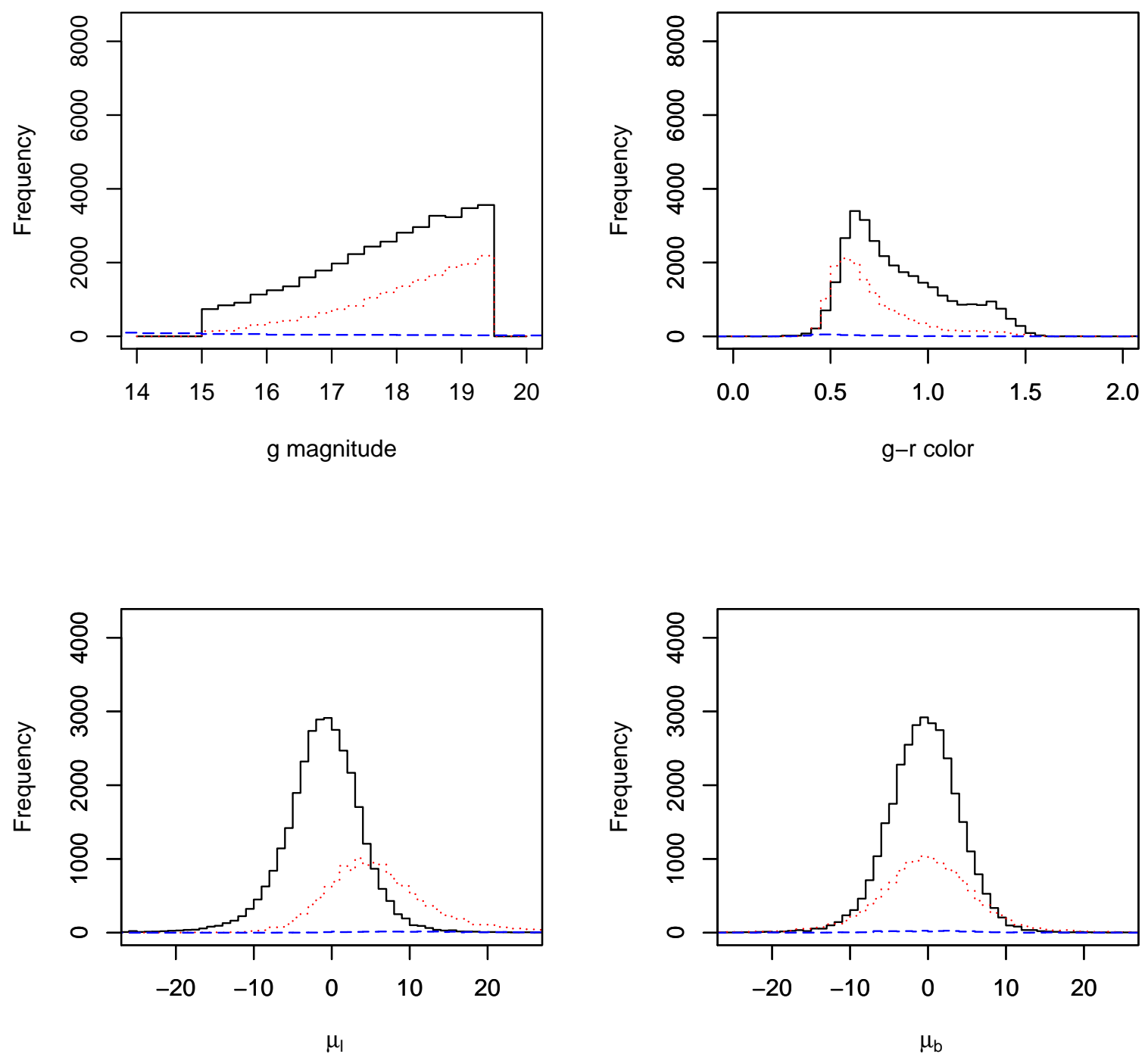

Figure 7.32: Magnitude, color (top panels) and proper motion distributions (bottom panels) separated by populations for field 2668. Thin disc stars: Black solid line; Thick disc stars: Red dotted line; Halo stars: Blue dashed line. 

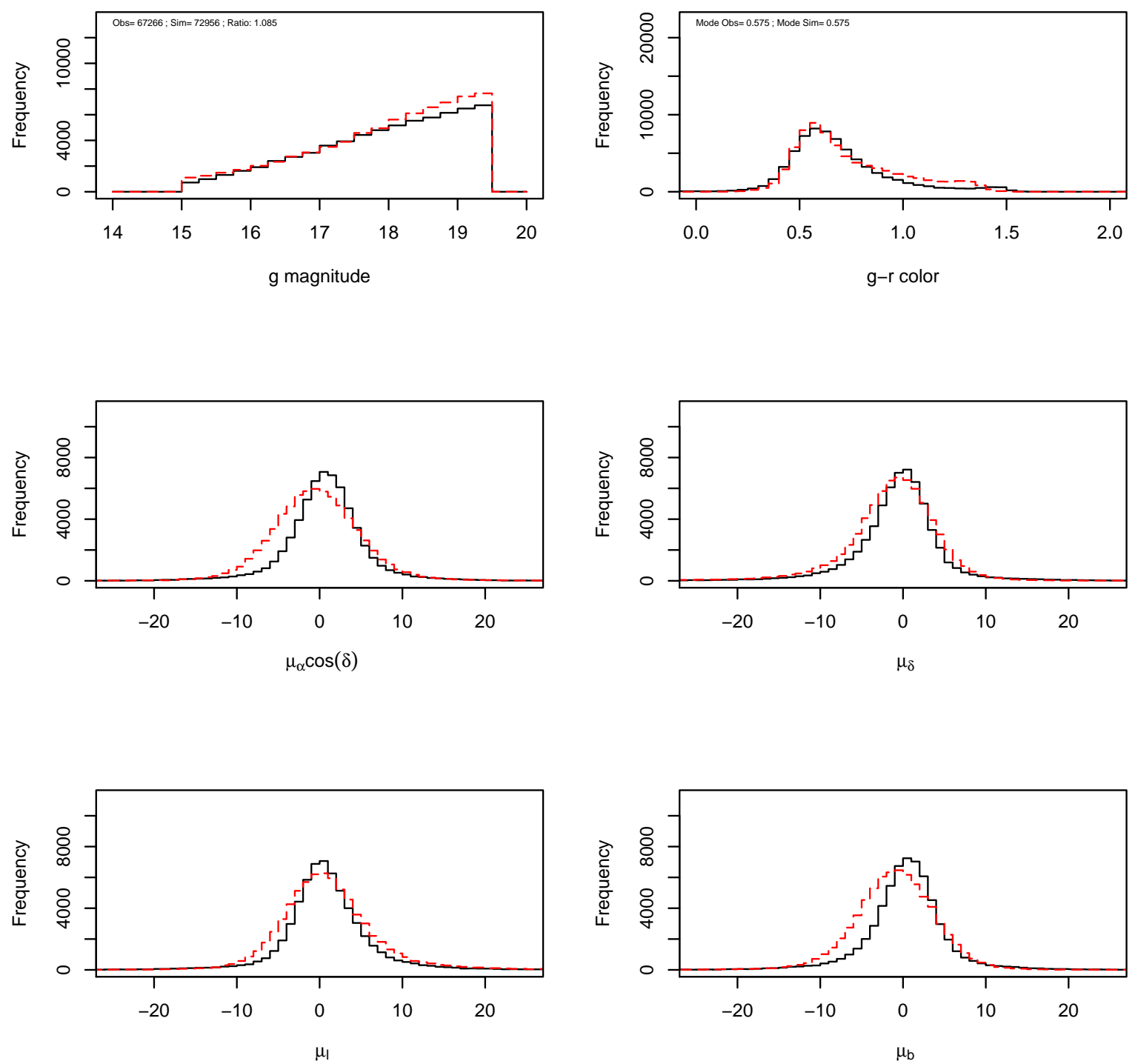

Figure 7.33: Magnitude, color (top panels) and proper motion distributions (middle and bottom panels) for field 2678. Black histograms are observations and the red histograms are simulations 

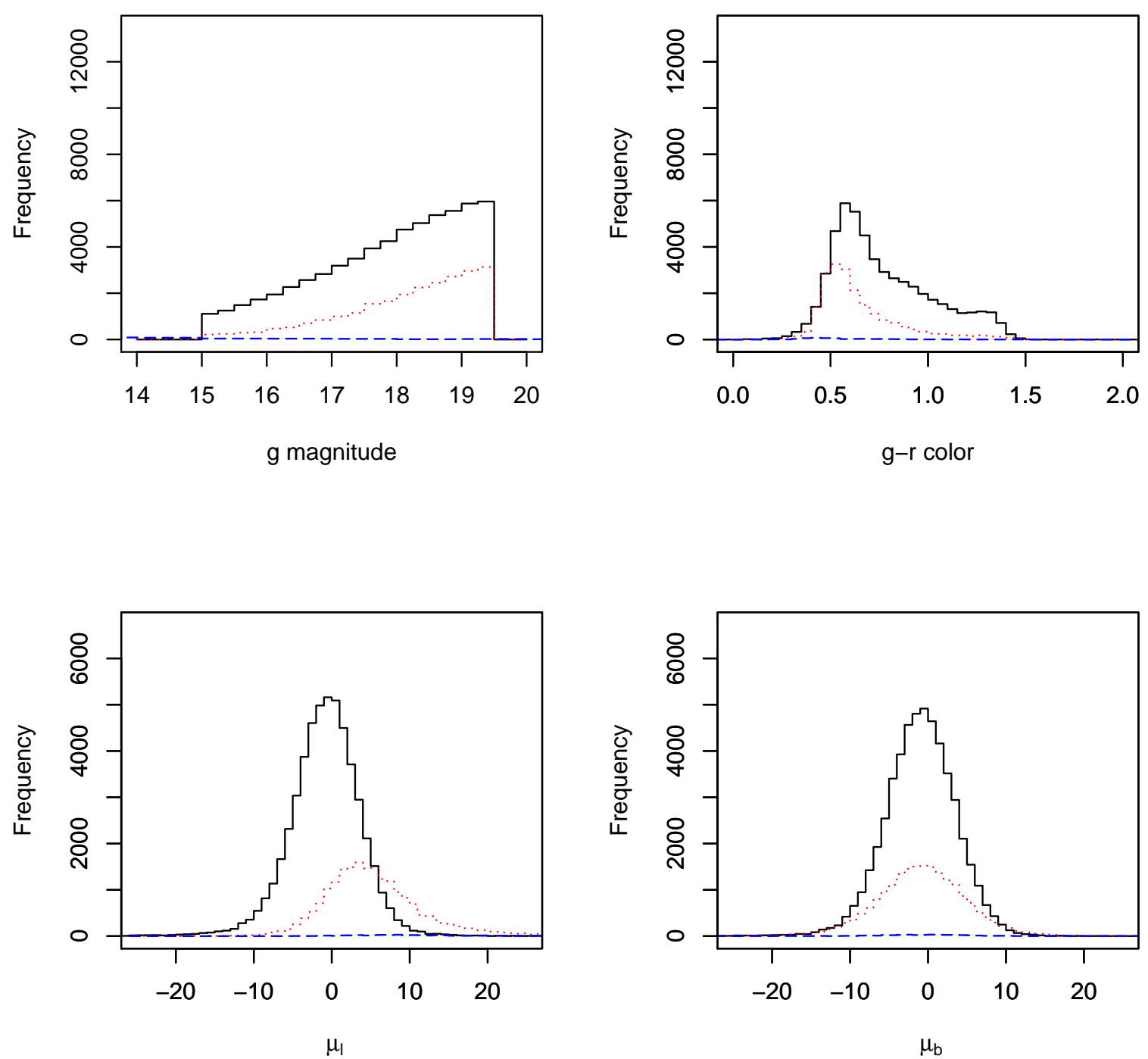

Figure 7.34: Magnitude, color (top panels) and proper motion distributions (bottom panels) separated by populations for field 2678. Thin disc stars: Black solid line; Thick disc stars: Red dotted line; Halo stars: Blue dashed line. 

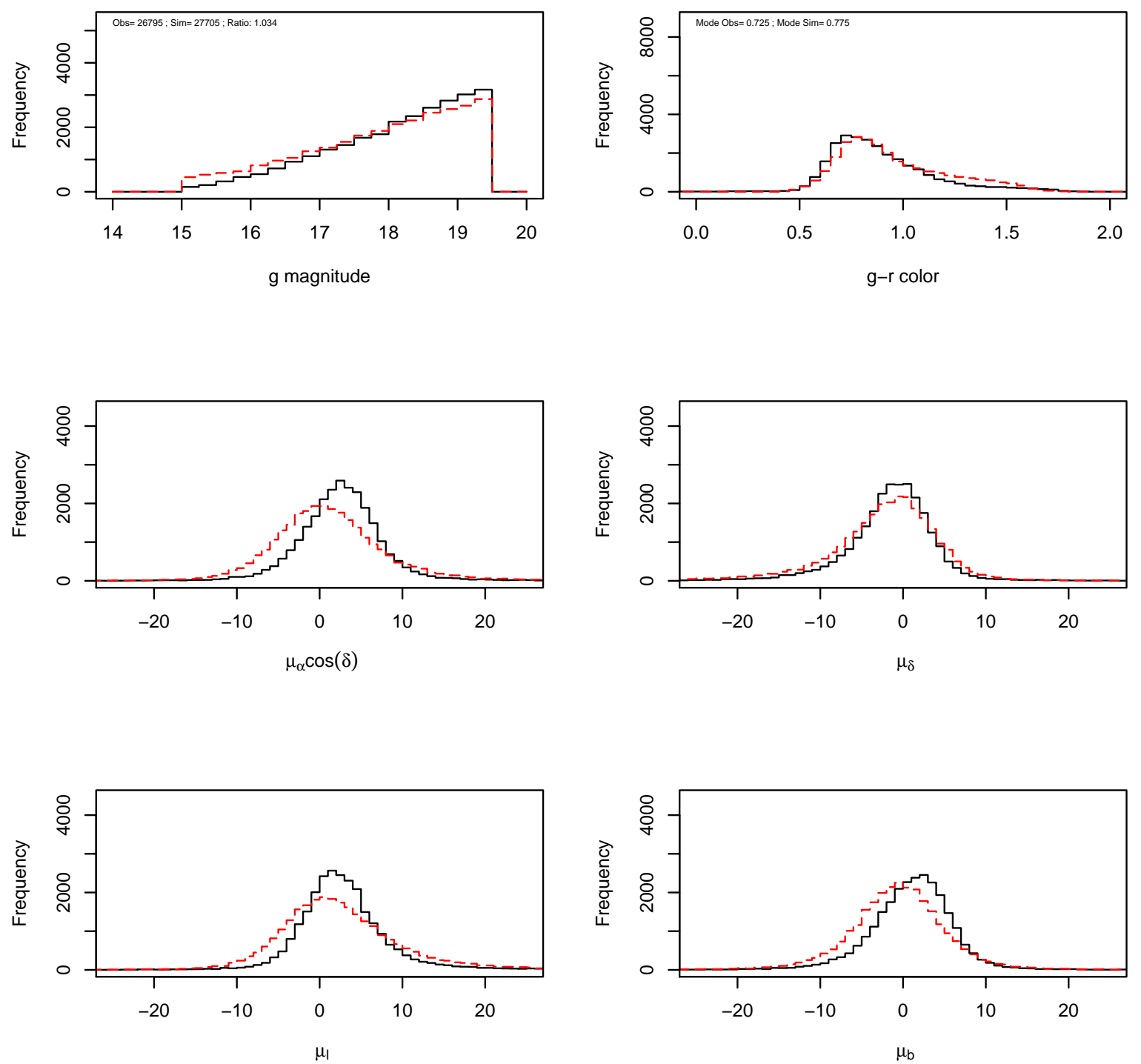

Figure 7.35: Magnitude, color (top panels) and proper motion distributions (middle and bottom panels) for field 2681. Black histograms are observations and the red histograms are simulations. 

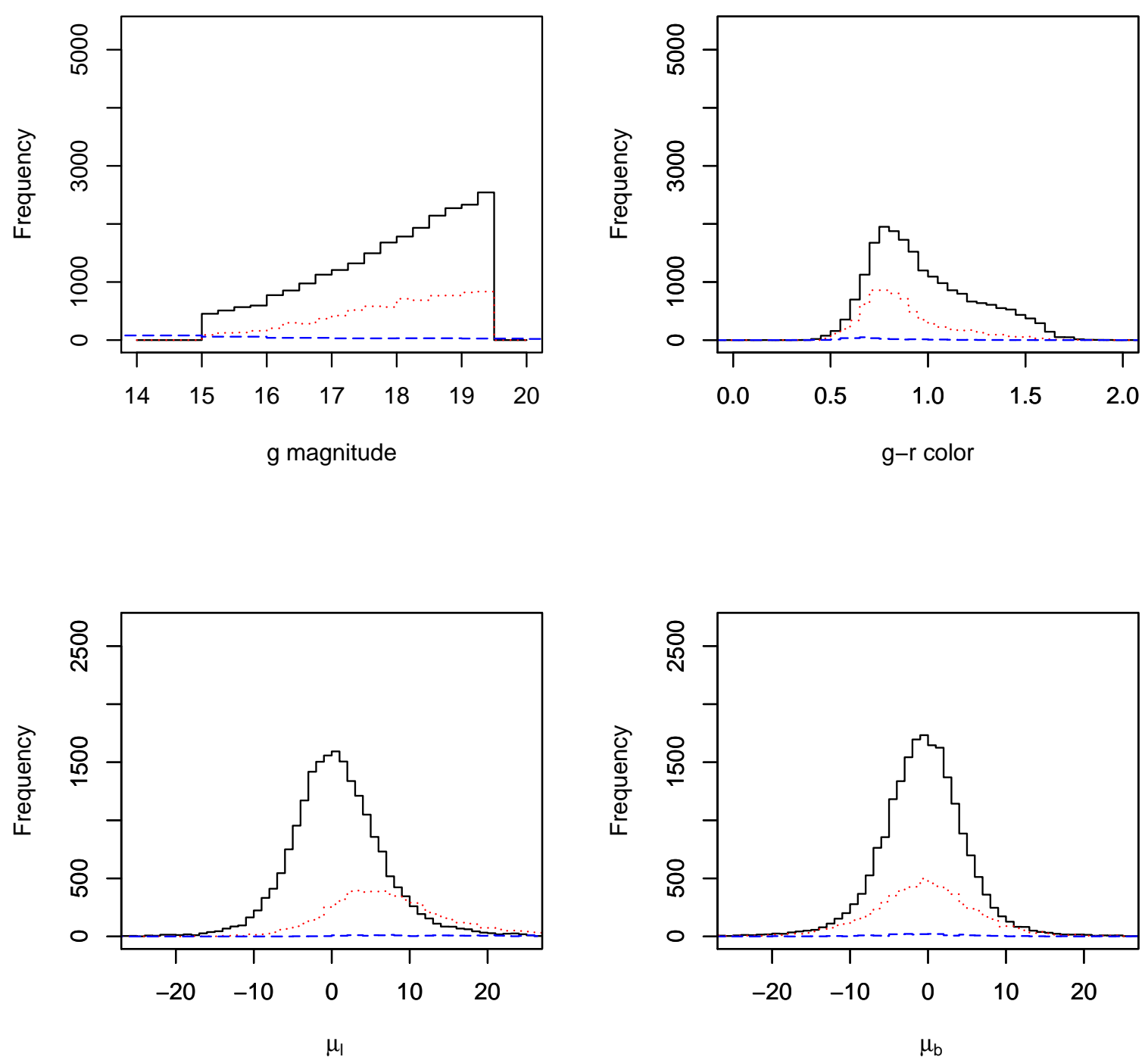

Figure 7.36: Magnitude, color (top panels) and proper motion distributions (bottom panels) separated by populations for field 2681. Thin disc stars: Black solid line; Thick disc stars: Red dotted line; Halo stars: Blue dashed line. 
Table 7.1:

Proper motions measures of central tendency (Mean and Mode) and of dispersion (Standard deviation) for the l-component

\begin{tabular}{|c|c|c|c|c|c|c|}
\hline Field & $\begin{array}{c}\operatorname{Mean}_{o b s} \\
\left({\left.\text { mas } y r^{-1}\right)}^{-1}\right)\end{array}$ & $\begin{array}{c}\operatorname{Mean}_{\text {sim }} \\
\left({\left.\text { mas } y r^{-1}\right)}\right.\end{array}$ & $\begin{array}{l}\text { Mode }_{o b s} \\
\left({\left.\text { mas } y r^{-1}\right)}\right.\end{array}$ & $\begin{array}{c}\text { Mode }_{\text {sim }} \\
\left({\left.\text { mas } y r^{-1}\right)}\right.\end{array}$ & $\begin{array}{c}\sigma_{o b s} \\
m a s y r^{-1}\end{array}$ & $\begin{array}{c}\sigma_{\operatorname{sim}} \\
\left(\operatorname{mas}_{y} r^{-1}\right)\end{array}$ \\
\hline 2534 & -1.77 & -5.33 & -2.35 & -5.45 & 9.04 & 8.46 \\
\hline 2536 & -2.46 & -4.81 & -2.76 & -4.52 & 8.34 & 9.02 \\
\hline 2537 & -0.34 & -2.38 & -1.24 & -3.15 & 9.32 & 9.02 \\
\hline 2538 & 0.03 & -1.30 & 0.27 & -3.11 & 11.41 & 14.50 \\
\hline 2554 & -1.87 & -3.22 & -2.27 & -4.05 & 8.29 & 9.99 \\
\hline 2555 & 0.31 & -3.65 & 0.11 & -4.30 & 9.29 & 10.52 \\
\hline 2556 & -0.46 & -3.87 & -0.63 & -3.76 & 7.54 & 7.92 \\
\hline 2668 & 2.09 & 1.52 & 1.14 & 0.20 & 6.84 & 9.55 \\
\hline 2678 & 0.93 & 1.20 & -0.18 & 0.52 & 8.48 & 8.13 \\
\hline 2681 & 3.11 & 2.44 & 1.87 & -0.08 & 8.73 & 11.66 \\
\hline
\end{tabular}

\subsubsection{Proper motions}

Tables 7.1 and 7.2 show the mean, mode and dispersion for the proper motion components 1 and $\mathrm{b}$ respectively. Tables show that the model reproduces the proper motion distribution, the mean, mode and dispersion. For an easier inspection of the tables we plot in figure 7.37 the proper motion measures of central tendency (Mean and Mode) and dispersion (Standard deviation) as a function of the galactic coordinates. Even if the overall distribution is reproduced, there is a slight shift in the 1 component of the proper motion. This slight shift is particularly clear in the top left panel where observations are systematically larger than simulations. The shifts in the l component of the proper motion depend on the longitude because the anticenter fields show a better agreement than the fields with shorter longitudes which means that the component $\mathrm{V}$, defined as the circular velocity of the UVW triad needs to be revised. The shifts do not depend on the galactic latitude.

The $\mathrm{b}$ component of the proper motion seem also to be shifted by a few amount that is particularly evident for the anticenter fields. It can be due to the presence of some structure or indicates that the $\mathrm{b}$ component of the proper motions needs also to be revised. Analysis and better constrains of the kinematics in the model are being done by other collaborators. 

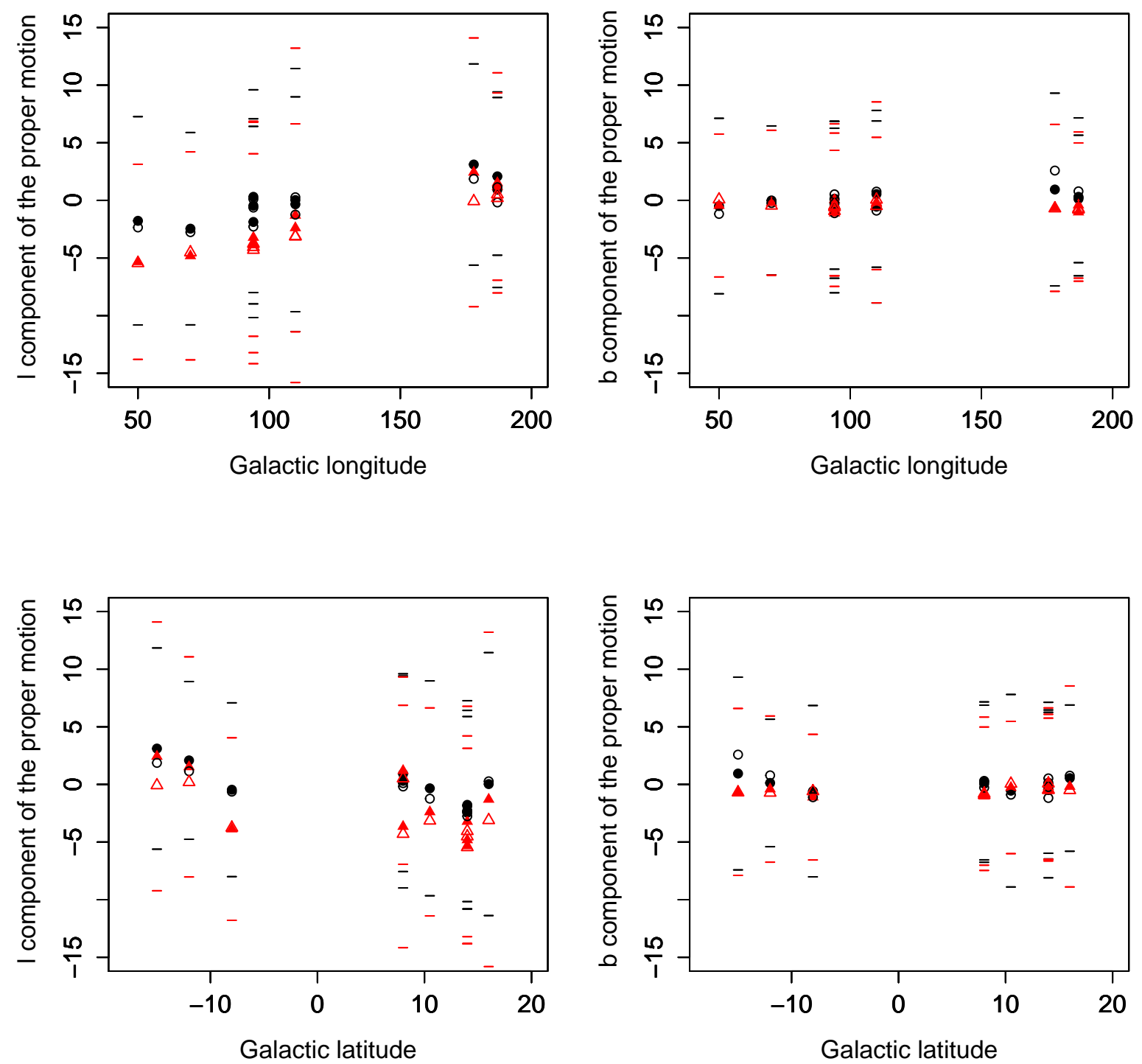

Figure 7.37: Proper motion (in mas $y r^{-1}$ ) measures of central tendency (Mean and Mode) and of dispersion (Standard deviation) as a function of the galactic coordinates. black filled circles: mean of the observations; black open circles: mode of the observations; red filled triangles:

mean of the simulations; red open triangles: mode of the simulations. Top panel: 1 and $b$ components of the proper motion as a function of the galactic longitude. Bottom panel: 1 and $b$ components of the proper motion as a function of the galactic latitude. 
Table 7.2:

Proper motions measures of central tendency (Mean and Mode) and of dispersion (Standard deviation) for the b-component

\begin{tabular}{ccccccc}
\hline \hline Field & $\begin{array}{c}\mathrm{Mean}_{\text {obs }} \\
\left(\text { mas yr }^{-1}\right)\end{array}$ & $\begin{array}{c}\mathrm{Mean}_{\text {sim }} \\
\left(\text { mas yr }^{-1}\right)\end{array}$ & $\begin{array}{c}\mathrm{Mode}_{\text {obs }} \\
\left(\text { mas yr }^{-1}\right)\end{array}$ & $\begin{array}{c}\mathrm{Mode}_{\text {sim }} \\
\left(\text { mas yr }^{-1}\right)\end{array}$ & $\begin{array}{c}\sigma_{\text {obs }} \\
\text { mas yr }\end{array}$ & $\begin{array}{c}\sigma_{\text {sim }} \\
\left({\left.\text { mas } y r^{-1}\right)}\right.\end{array}$ \\
\hline 2534 & -0.49 & -0.45 & -1.18 & 0.08 & 7.61 & 6.20 \\
2536 & -0.01 & -0.21 & -0.23 & -0.46 & 6.46 & 6.29 \\
2537 & -0.55 & -0.27 & -0.89 & 0.06 & 8.35 & 5.74 \\
2538 & 0.55 & -0.18 & 0.76 & -0.50 & 6.34 & 8.72 \\
2554 & 0.14 & 0.05 & 0.53 & -0.48 & 6.11 & 6.59 \\
2555 & 0.06 & -0.81 & -0.28 & -0.93 & 6.81 & 6.65 \\
2556 & -0.59 & -1.10 & -1.11 & -0.59 & 7.43 & 5.45 \\
2668 & 0.13 & -0.41 & 0.79 & -0.72 & 5.53 & 6.34 \\
2678 & 0.31 & -1.01 & 0.24 & -0.80 & 6.85 & 6.00 \\
2681 & 0.94 & -0.65 & 2.59 & -0.70 & 8.36 & 7.24 \\
\hline \hline
\end{tabular}

\subsection{Constraining the Initial Mass Function}

\subsubsection{Method}

The Initial mass function (IMF), is the distribution in mass of the stars at their birth. The IMF is assumed to follow power laws by intervalle of masses. The slopes and mass ranges are given in table 3.1. We developed a program to constrain the IMF slope in the thin disc. We do not include field $2555\left((1, b)=\left(94^{\circ}, 8^{\circ}\right)\right)$ due to the large difference in star counts between observations and simulations. The 9 fields have 608421 stars. This program tries to find the best IMF slope doing a $\chi^{2}$ minimization of the star counts for bins of magnitude $\mathrm{g}(0.5 \mathrm{mag})$ and color $\mathrm{g}-\mathrm{r}$ ( 0.05 mag). The $\chi^{2}$ is defined by

$$
\chi^{2}=\sum_{i} \frac{1}{N-1} \frac{O_{i}-E_{i}}{E_{i}}
$$

where $O_{i}$ and $E_{i}$ are respectively the observed and simulated number of stars in bin i and $\mathrm{N}$ is the number of bins. Each time we test a new IMF slope the number of stars in the simulated bins has to change. For each star we give a weigth $\omega$ that is equal to the difference between the assumed IMF in the model and the IMF slope to test.

$$
\omega(m)=m^{\left(I M F_{[o r i g i n a l]}-I M F_{[t e s t]}\right)}
$$




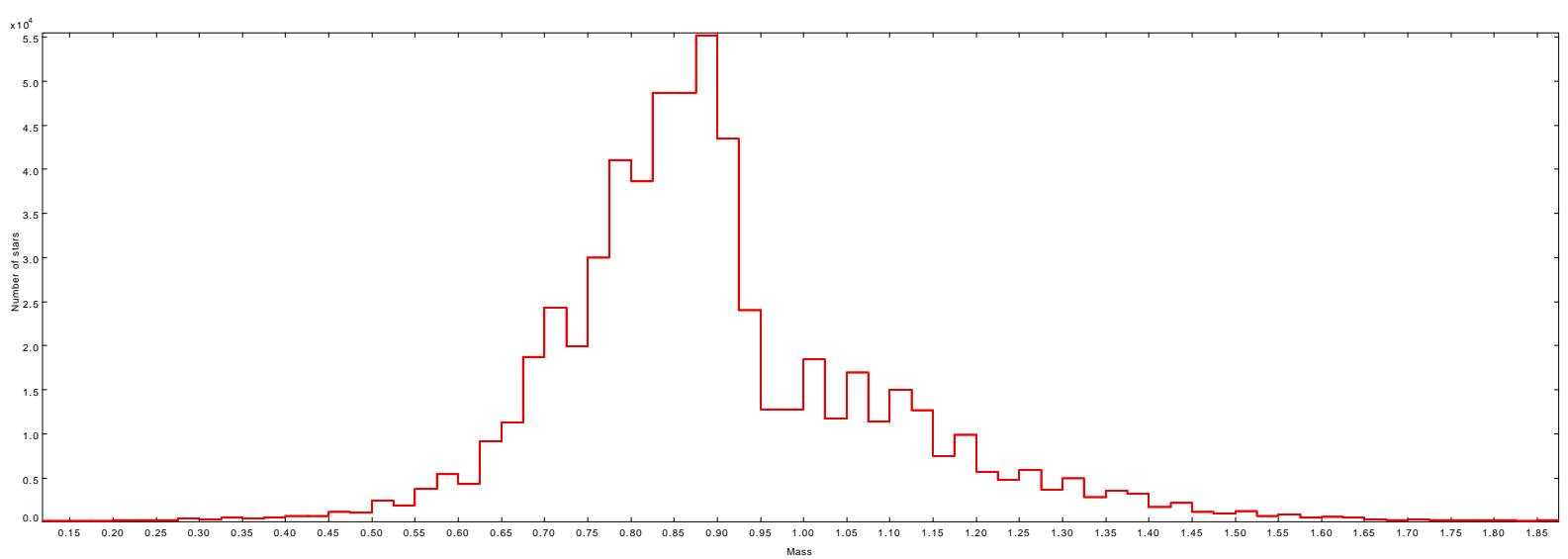

Figure 7.38: Mass distribution for the fields used to fit the IMF as given by the standard model.

We fill the color magnitude bins with the computed weights. The IMF slope $(\alpha=-(x+1))$ is allowed to vary between zero and one for stars in the thin disc where the mass ranges from 0.5 to 1.53 solar masses. Figure 7.38 shows the range of masses at which we are sensible.

\subsubsection{Results}

The best result for the IMF slope was equal to 0.58 , for $\chi^{2} \approx 46.86$ (The original one is $\chi^{2} \approx$ 46.88). Figure 7.39 shows the $\chi^{2}$ values as a function of the IMF slope. Although we have a minimum value the small (in the decimal case) variation of the $\chi^{2}$ values, for different IMF slopes, is not significant as seen in figure 7.39 . We have tried to change the mass range $(0.5$ to 1.0 and 1.0 to 1.53 ) where we want to constrain the IMF slope but the results did not change. We have included, in our fit, the IMF of the thick disc as a variable but results were not obtained. An explanation is that for most of the fields the agreement is already very good and if it not good it is due to something else than the IMF slope. The lesson to learn here is that the IMF is not playing an important role. It seems that these data and this particular range of magnitudes are not much sensitive to the IMF but rather to quantities like the scale length or the warp of the disc. 


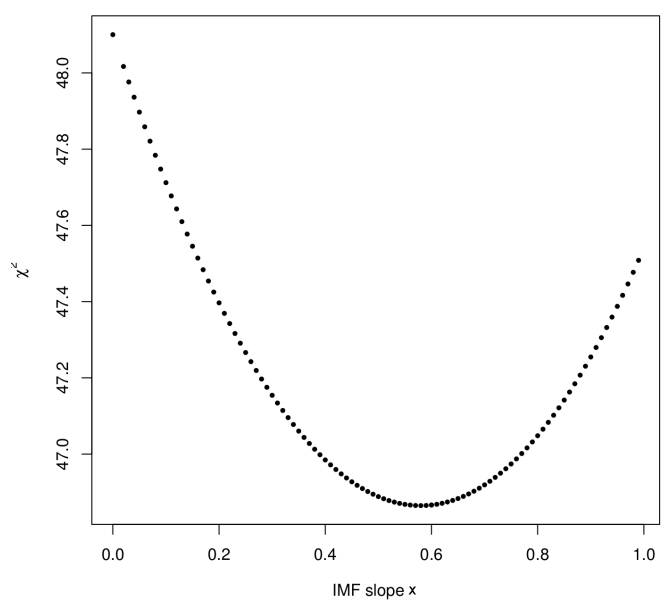

Figure 7.39: $\chi^{2}$ as a function of the IMF slopes.

\subsection{Comparison with a new version of the Besançon galaxy model}

We compare model A and model B of the revised version of the Besancon Galaxy Model (Czekaj et al. 2014), where more flexibility is possible for testing variable SFR history, IMF, and which takes into account a complete treatment of the binarity, with data. We have compared the standard model with two versions (model A and model B) of the revised version explained in section 3.4. We have done 10 simulations and we compute the mean $\chi^{2}$ for each parameter. The $\sigma$ is computed from the standard deviation of the 10 runs and is given for each spectroscopic parameter. The results are given in table 7.3. The degrees of freedom for magnitude, color and color-magnitude are given in table 7.4.

This table shows that the standard model and model B of the revised model are the best models. The Figures 7.40, 7.41 and 7.42 show the $\chi^{2}$ values for the magnitude, color and color magnitude respectively in each field for the original and model B as a function of the galactic longitude. The color code is the galactic latitude. The table and figures indicate that the magnitude distribution for both models is compatible inside errors while for the color and color magnitude distribution model B is slightly better. Model B results are better for the anti center fields and it seems to be slightly better for fields near the plane except field 2555 (See fields with galactic latitudes lower or equal to $14^{\circ}$ ).

In general model A results show a slightly larger $\chi^{2}$ value compared with model B but results are very similar and inside errors. In the anticenter directions model A is even slightly worst than the original model. In the intermediate longitudes model A is compatible with model B and shows the same galactic latitude pattern already explained above for model B. 
Table 7.3:

$\chi^{2}$ values (magnitude $(\mathrm{m})$, color(c) and color-magnitude $(\mathrm{c}-\mathrm{m})$ ) along with dispersion (for the standard model). Last three lines show the sum of $\chi^{2}$ values for all fields.

\begin{tabular}{|c|c|c|c|c|c|c|c|c|c|}
\hline Model & field & $\chi^{2}(\mathrm{~m})$ & $\sigma$ & $\chi^{2}(\mathrm{c})$ & $\sigma$ & $\chi^{2}(\mathrm{c}-\mathrm{m})$ & $\sigma$ & $1(\mathrm{deg})$ & $\mathrm{b}(\mathrm{deg})$ \\
\hline Original & 2534 & 3265.44 & 292.20 & 21039.85 & 2154.79 & 28835.01 & 2687.61 & 50 & 14 \\
\hline Model A & 2534 & 6320.42 & 653.65 & 20919.18 & 3045.92 & 30900.25 & 2989.88 & & \\
\hline Model B & 2534 & 6647.83 & 339.59 & 20766.18 & 2302.89 & 31113.17 & 2758.46 & & \\
\hline Original & 2536 & 1697.94 & 92.01 & 9959.42 & 475.77 & 15467.01 & 578.73 & 70 & 14 \\
\hline Model A & 2536 & 1935.88 & 227.97 & 8284.36 & 1283.17 & 13368.85 & 1424.70 & & \\
\hline Model B & 2536 & 1639.00 & 107.00 & 7471.17 & 441.14 & 12256.37 & 504.47 & & \\
\hline Original & 2537 & 1836.61 & 156.51 & 4408.88 & 556.79 & 8282.27 & 553.98 & 110 & 10.5 \\
\hline Model A & 2537 & 1710.19 & 331.36 & 2480.89 & 275.28 & 6678.06 & 244.29 & & \\
\hline Model B & 2537 & 1501.28 & 206.74 & 2336.22 & 176.35 & 6009.54 & 389.89 & & \\
\hline Original & 2538 & 2146.81 & 188.00 & 1781.97 & 161.72 & 5522.17 & 650.38 & 110 & 16 \\
\hline Model A & 2538 & 1838.94 & 211.77 & 1690.52 & 351.36 & 5533.93 & 864.50 & & \\
\hline Model B & 2538 & 2125.74 & 182.68 & 2027.34 & 173.12 & 5568.46 & 526.91 & & \\
\hline Original & 2554 & 316.90 & 41.46 & 5459.37 & 307.75 & 9508.80 & 401.40 & 94 & 14 \\
\hline Model A & 2554 & 363.37 & 34.28 & 3544.08 & 390.68 & 7115.28 & 511.95 & & \\
\hline Model B & 2554 & 253.73 & 29.68 & 3368.69 & 366.52 & 6611.06 & 433.12 & & \\
\hline Original & 2555 & 8825.03 & 727.44 & 7768.20 & 920.26 & 17436.47 & 2177.05 & 94 & 8 \\
\hline Model A & 2555 & 10557.53 & 1211.90 & 10164.74 & 1314.28 & 18775.38 & 2167.23 & & \\
\hline Model B & 2555 & 11847.37 & 840.84 & 11327.78 & 708.97 & 18551.59 & 1324.34 & & \\
\hline Original & 2556 & 4439.79 & 385.20 & 20053.89 & 635.30 & 27883.31 & 832.51 & 94 & -8 \\
\hline Model A & 2556 & 3209.40 & 1143.53 & 13923.36 & 2141.59 & 18845.17 & 1621.52 & & \\
\hline Model B & 2556 & 1863.66 & 86.28 & 9226.29 & 629.56 & 15088.31 & 849.18 & & \\
\hline Original & 2668 & 5127.29 & 424.42 & 7925.56 & 420.67 & 15888.61 & 1046.29 & 187 & -12 \\
\hline Model A & 2668 & 6430.66 & 392.83 & 9440.03 & 1350.76 & 19693.12 & 2333.91 & & \\
\hline Model B & 2668 & 3757.83 & 374.96 & 6823.93 & 826.05 & 14581.14 & 1380.03 & & \\
\hline Original & 2678 & 1015.57 & 83.38 & 14585.23 & 461.63 & 23804.41 & 539.95 & 187 & 8 \\
\hline Model A & 2678 & 1911.33 & 1592.96 & 7868.66 & 465.81 & 28462.09 & 2749.10 & & \\
\hline Model B & 2678 & 548.01 & 43.62 & 6201.01 & 320.24 & 26934.50 & 1361.78 & & \\
\hline Original & 2681 & 1921.90 & 149.13 & 3144.32 & 284.41 & 7997.68 & 392.73 & 178 & -15 \\
\hline Model A & 2681 & 2565.13 & 248.59 & 5994.60 & 993.20 & 11098.42 & 1181.17 & & \\
\hline Model B & 2681 & 1356.96 & 178.74 & 3409.18 & 573.70 & 7530.02 & 754.41 & & \\
\hline Original & All & 30593.28 & 2539.75 & 96126.69 & 6379.08 & 160625.76 & 9860.63 & & \\
\hline Model A & All & 36842.86 & 6048.83 & 84310.42 & 11612.04 & 160470.55 & 16088.24 & & \\
\hline Model B & All & 31541.42 & 2390.14 & 72957.79 & 6518.54 & 144244.16 & 10282.60 & & \\
\hline
\end{tabular}


Table 7.4:

Deegres of freedom (df) for magnitude(m), color(c) and color-magnitude(c-m).

\begin{tabular}{cccc}
\hline \hline Field & df(m) & df(c) & df(c-m) \\
\hline 2534 & 44 & 36 & 1336 \\
2536 & 44 & 34 & 1305 \\
2537 & 44 & 38 & 1175 \\
2538 & 44 & 37 & 1040 \\
2554 & 44 & 34 & 1268 \\
2555 & 44 & 37 & 1409 \\
2556 & 44 & 36 & 1322 \\
2668 & 44 & 37 & 1181 \\
2678 & 44 & 34 & 1234 \\
2681 & 44 & 39 & 1152 \\
& & & \\
\hline \hline
\end{tabular}

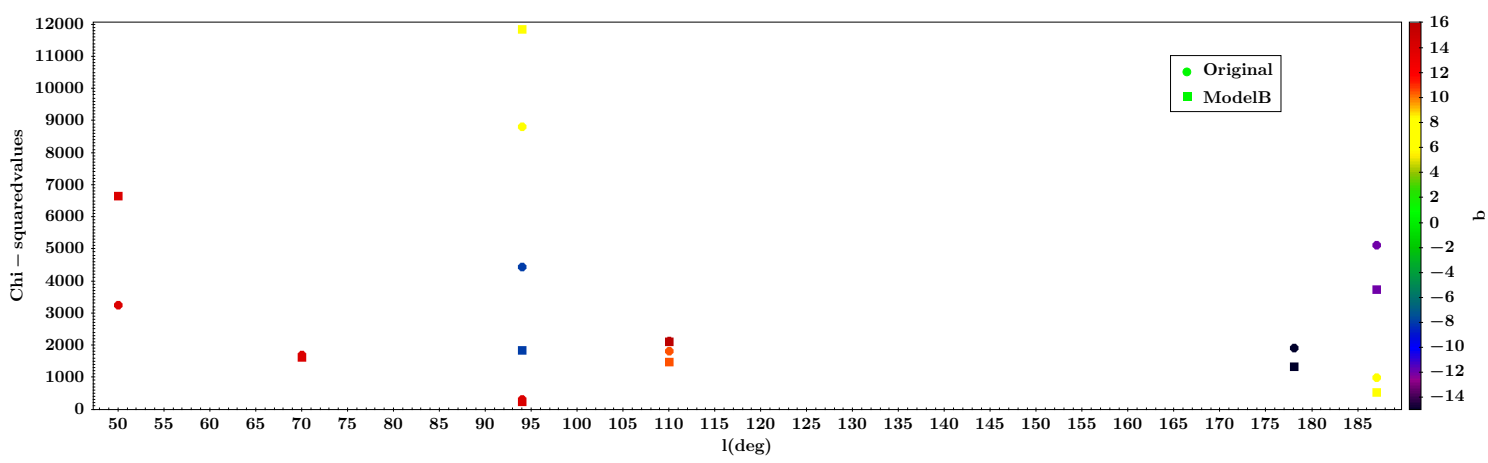

Figure 7.40: $\chi^{2}$ for the magnitude distribution as a function of galactic longitude. The color coding is galactic latitude. Circles are the original simulation and squares are model B of the revised version of the BGM.

These comparisons between the original and revised model have to be done but in general the revised model seems to describe better the magnitude and color distributions near the plane when compared to the original model. To confirm these results a comparison using more data sets in the plane should be done. 


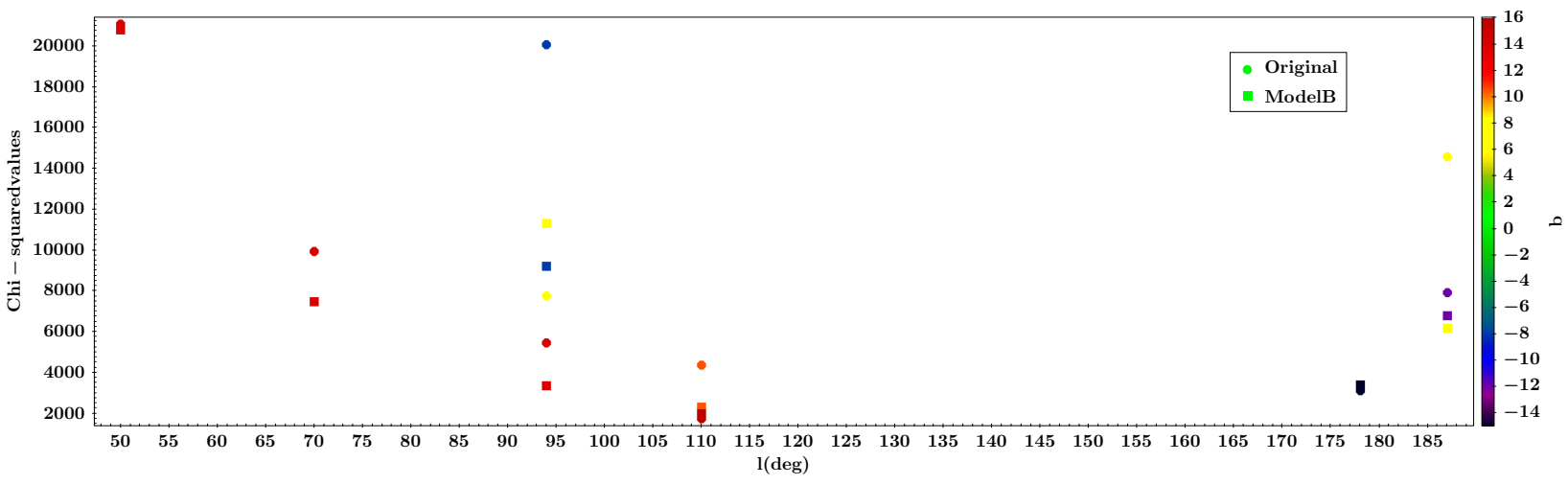

Figure 7.41: $\chi^{2}$ for the color distribution as a function of galactic longitude. The color coding is galactic latitude. Circles are the original simulation and squares are model B of the revised version of the BGM.

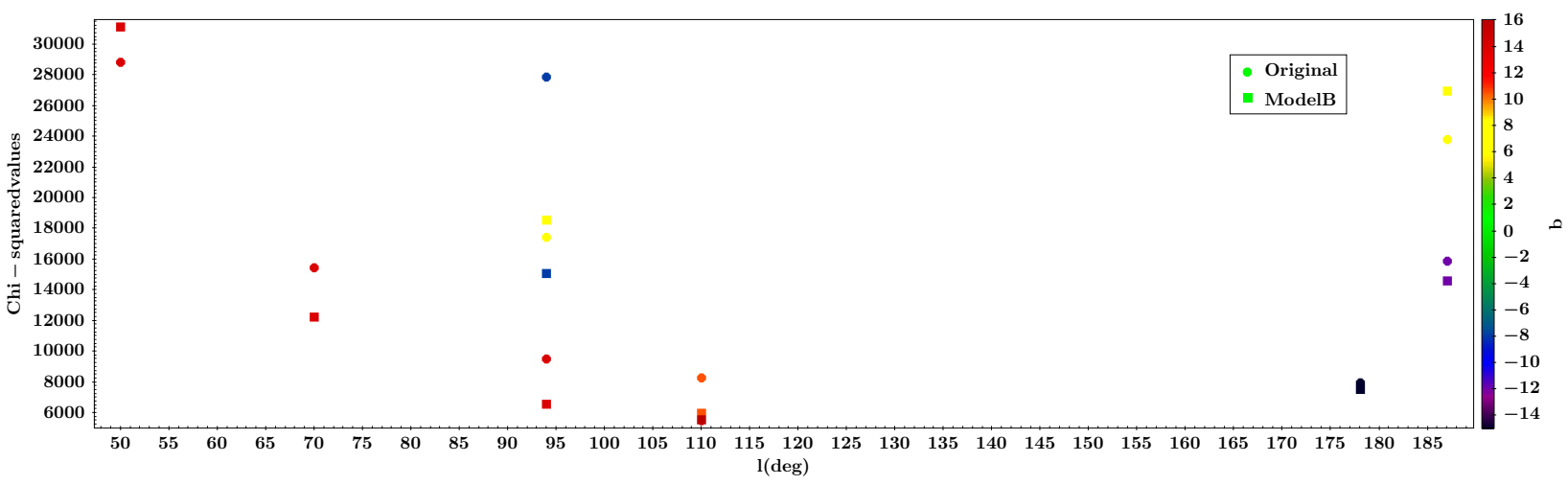

Figure 7.42: $\chi^{2}$ for the color magnitude distribution as a function of galactic longitude. The color coding is galactic latitude. Circles are the original simulation and squares are model B of the revised version of the BGM. 


\section{Chapter 8}

\section{Spectroscopic results from the SEGUE low latitude data}

In this section we present the work done with the spectroscopic samples selected as explained in section 5. Section 8.1 describes the method, discussion and conclusions for analysis made to the Main Sequence Turnoff stars sample while section 8.3 describes the results obtained for the $\mathrm{K}$ giants sample.

\subsection{Main Sequence Turn off stars}

\subsubsection{Comparison between observations and simulations}

In Table 8.1 we show the proportion of thin to thick disc stars, selected from the two populations in the model, for each field in the simulated catalogs. The mass range in the thin disc, for the selected sample, is between $0.9 M_{\odot}$ and $1.5 M_{\odot}$ and for the thick disc is between $0.7 M_{\odot}$ and $0.95 M_{\odot}$. The sample covers a galactocentric distance in the range $6.0<R_{\text {gal }}<15 \mathrm{kpc}$ and a distance above the plane in the range $-1.5<Z<1.5 \mathrm{kpc}$ as shown in figure 8.1.

\subsubsection{Preliminary comparison with the standard model}

We compared the simulated spectral parameters distributions with observed spectrocopic distributions.

Figure 8.2 and 8.3 show, bright and faint plates respectively from field 2537 towards $l=$ $110^{\circ}$ and $b=10.5^{\circ}$, comparisons for three parameters: The metallicity, the temperature and the gravity. The comparison regarding the faint plate of the same field is done in Figure 8.3. 
Table 8.1:

The number of stars of the thick disc/thin disc in the standard model in each plate for the bright (b) and faint (f) plates after applying the selection sample. The number of stars in each plate and the mode of the total extinction $A_{V}$ for each distribution are also indicated.

\begin{tabular}{cccccccc}
\hline \hline Plate & thin/thick (b) & thin/thick (f) & $N^{o}$ of stars (b) & $N^{o}$ of stars (f) & $A_{V}(\mathrm{mag})$ & $1\left(^{\circ}\right)$ & $\mathrm{b}\left({ }^{\circ}\right)$ \\
\hline 2534 & $75 / 102$ & $20 / 249$ & 181 & 285 & 0.605 & 50 & 14 \\
2536 & $67 / 109$ & $29 / 256$ & 183 & 314 & 0.550 & 70 & 14 \\
2537 & $129 / 59$ & $156 / 146$ & 190 & 304 & 0.961 & 110 & 10.5 \\
2538 & $201 / 85$ & $134 / 192$ & 288 & 329 & 1.764 & 110 & 16 \\
2554 & $91 / 125$ & $48 / 262$ & 222 & 332 & 0.691 & 94 & 14 \\
2555 & $153 / 24$ & $128 / 69$ & 177 & 199 & 1.852 & 94 & 8 \\
2556 & $159 / 45$ & $148 / 153$ & 209 & 302 & 0.848 & 94 & -8 \\
2668 & $201 / 73$ & $211 / 180$ & 276 & 393 & 0.683 & 187 & 14 \\
2678 & $170 / 61$ & $205 / 115$ & 232 & 322 & 0.519 & 187 & 8 \\
2681 & $177 / 93$ & $166 / 189$ & 276 & 361 & 0.918 & 178 & -15 \\
\hline \hline
\end{tabular}

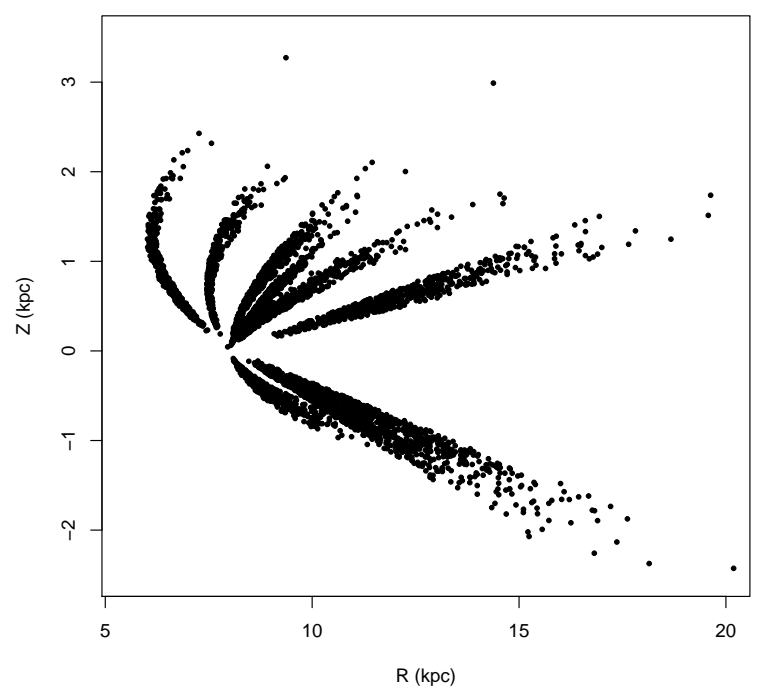

Figure 8.1: Distance from the plane as a function of the galactocentric distance for the MSTO stars sample as given by the simulations. 

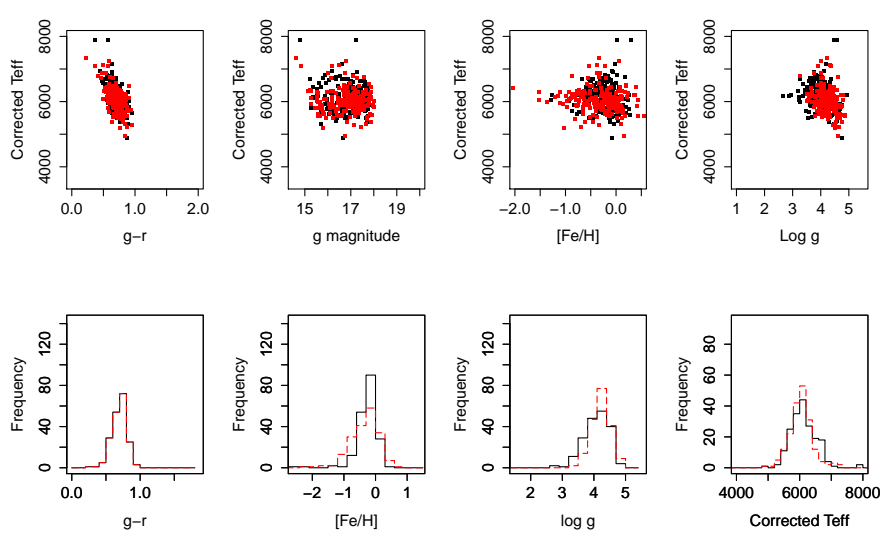

Figure 8.2: Comparison of the spectrocopic observations and simulations for the bright plate $2537, l=110^{\circ}$ and $b=10.5^{\circ}$. Black points and lines are observations. Simulations are in red.
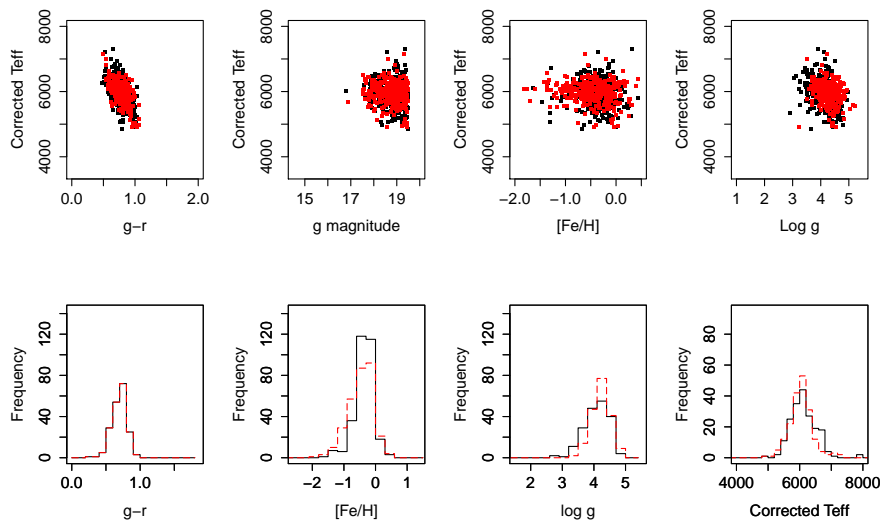

Figure 8.3: Comparison of the spectrocopic observations and simulations for the faint plate $2545, l=110^{\circ}$ and $b=10.5^{\circ}$. Black points and lines are observations. Simulations are in red.

We noticed that the simulated metallicity distribution has a stronger low metallicity tail than the observations. The fields we use in this work have a significant contribution of thick disc stars (see Table 8.1), so the metallicity distribution assumed in the model for this population should be revised. It suggests that the thick disc mean metallicity assumed in the model $([\mathrm{Fe} / \mathrm{H}]=-0.78$ dex) may be too low. In other fields the metallicity distribution is similar. For the temperature and gravity the agreement between simulations and observations is acceptable.

Table 8.2 shows likelihood of the simulations with regard to observations for different spectral parameters. The bright plates and faint plates are treated together. In order to estimate the goodness of fit of the model which will be used for improving the fit, we compute a likelihood. The goodness of fit estimation values are computed from the Bienaymé et al. (1987) reduced log likelihood formula for a binomial statistics (Kendall \& Stuart 1973). 
Table 8.2:

Likelihood values for the spectroscopic parameters (MSTO stars) - original model

\begin{tabular}{ccccccccccc}
\hline \hline Plate & {$[\mathrm{Fe} / \mathrm{H}]$} & $\sigma$ & $\mathrm{T}_{\text {eff }}$ & $\sigma$ & $\log g$ & $\sigma$ & $\mathrm{RV}$ & $\sigma$ & 1 & $\mathrm{~b}$ \\
\hline 2534 & -90.72 & 17.65 & -43.28 & 11.43 & -55.63 & 14.57 & -27.26 & 10.17 & 50 & 14 \\
2536 & -122.71 & 13.85 & -32.64 & 6.77 & -17.82 & 6.16 & -69.99 & 13.29 & 70 & 14 \\
2537 & -119.41 & 25.92 & -43.36 & 10.71 & -54.73 & 8.43 & -96.56 & 13.75 & 110 & 10.5 \\
2538 & -154.28 & 24.88 & -73.68 & 19.62 & -19.42 & 5.02 & -141.21 & 17.69 & 110 & 16 \\
2554 & -177.70 & 16.80 & -14.04 & 4.54 & -45.65 & 11.26 & -50.62 & 8.52 & 94 & 14 \\
2555 & -52.99 & 12.82 & -81.73 & 17.72 & -22.22 & 6.19 & -151.81 & 22.52 & 94 & 8 \\
2556 & -140.26 & 14.39 & -31.77 & 4.98 & -13.24 & 4.84 & -101.26 & 13.79 & 94 & -8 \\
2668 & -182.22 & 20.12 & -145.57 & 17.00 & -84.24 & 16.72 & -71.16 & 21.51 & 187 & -12 \\
2678 & -100.43 & 19.95 & -81.50 & 8.50 & -58.61 & 16.39 & -48.09 & 7.76 & 187 & 8 \\
2681 & -143.03 & 21.35 & -165.20 & 21.17 & -71.78 & 10.70 & -61.28 & 10.87 & 178 & -15 \\
\hline \hline
\end{tabular}

$$
L_{r}=\sum_{i=1}^{N} q_{i}\left(1-R_{i}+\ln \left(R_{i}\right)\right)
$$

where $\mathrm{i}$ is the number of bins and $R_{i}=f_{i} / q_{i}$ is the ratio between the number of stars in bin $\mathrm{i}$ in the model $\left(f_{i}\right)$ and in the data $\left(q_{i}\right)$.We compute ten realizations of each model and compute the mean and dispersion of the likelihood obtained from each realization.

\subsubsection{Metallicity variation with longitudes and latitudes}

Figure 8.4 shows the metallicity modes along the galactic longitude. In black are observations and in red simulations for the original model before fitting. We used the mode rather than the mean because it is more robust in case of skewed distribution. It is visible from the figure that the fields at $l=50^{\circ}$ and $l=70^{\circ}$ show lower metallicities in comparison with the ones at intermediate longitudes. It is even more visible for the faint plates. We have looked at the $\mathrm{S} / \mathrm{N}$ for the inner galaxy fields. The plate 2534, towards $l=50^{\circ}$ and $b=14^{\circ}$, has a lower $\mathrm{S} / \mathrm{N}$ compared with the remaining fields but still above 15 , which is sufficient to retrieve robust spectral parameters from the SSPP (Lee et al. 2008a). 

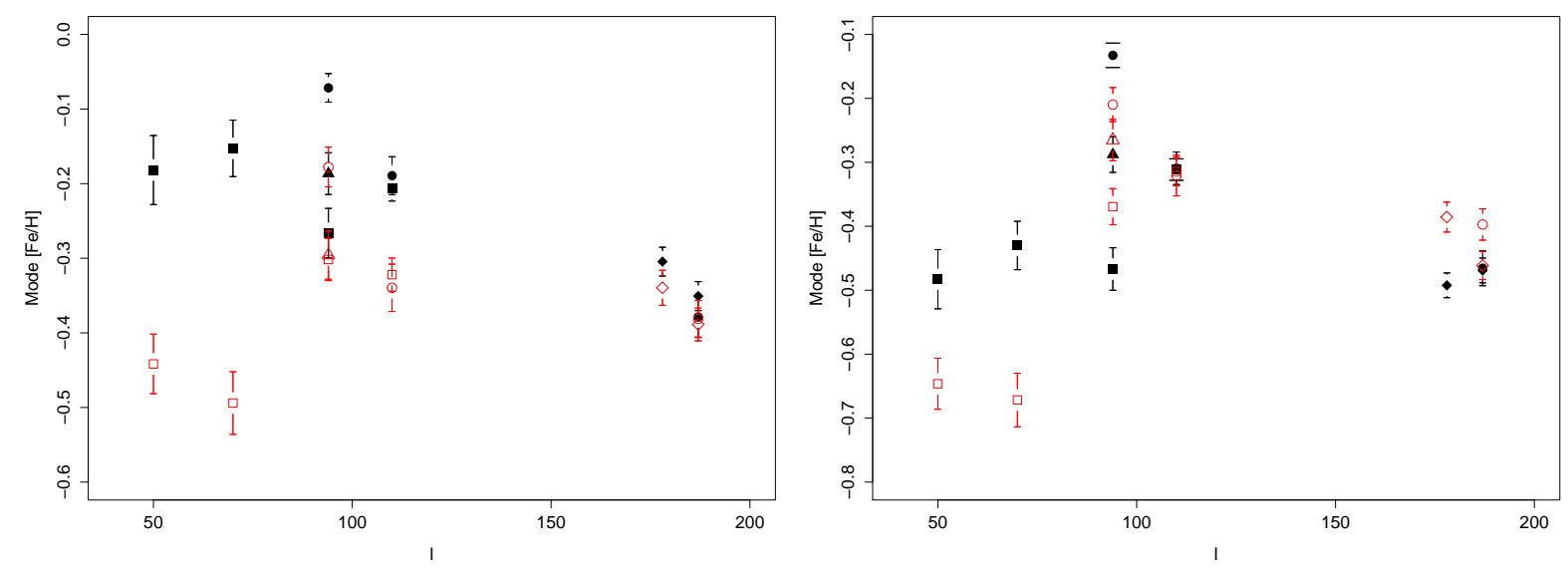

Figure 8.4: Mode of metallicities at different longitudes for the data and the standard model. left panel are the bright plates and right panel are the faint plates. The observations are in black and the simulations in red. The standard deviation is represented by the small bars. Squares are latitudes higher or equal to $14^{\circ}$; Circles are latitudes between $8^{\circ}$ and $10.5^{\circ}$; Triangles are latitudes equal to $-8^{\circ}$; Diamonds correspond to latitudes $-12^{\circ}$ and $-15^{\circ}$.

\subsubsection{Distances}

For this work we are not interested in computing the distances in a completely unbiased way, but in having a relative distance estimator with same biases in the simulated catalogue and in the observations. In the simulated catalogue, because we selected main sequence stars, there is a relation between effective temperature and absolute magnitude. The relation is metallicity dependent so we have divided the sample in three metallicity ranges:

- $[\mathrm{Fe} / \mathrm{H}] \leq-0.5$

- $-0.5<[\mathrm{Fe} / \mathrm{H}]<0.0$

- $[\mathrm{Fe} / \mathrm{H}] \geq 0.0$

For the two first metallicity ranges we estimate absolute magnitude as a function of temperature as a second order polynomial based on model simulations. For the third metallicity range we fitted a simple first order because the second order coefficient is null. Figure 8.5 shows the relations for the three ranges of metallicities. The fitted relations are given in equation 8.2. 

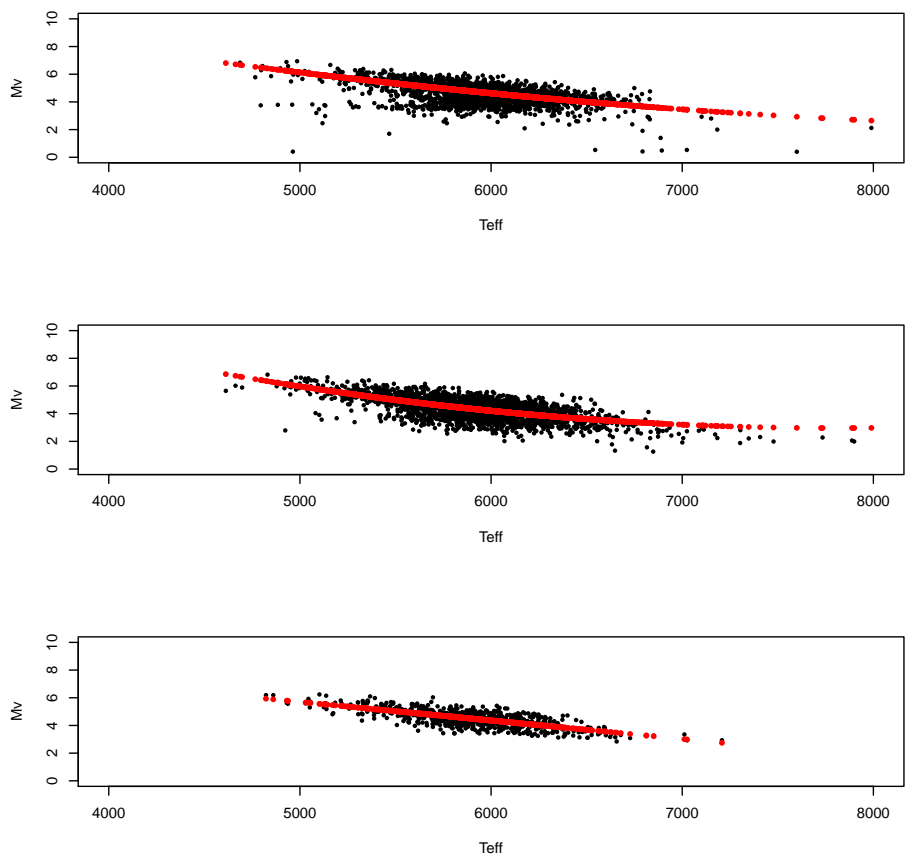

Figure 8.5: Relation between effective temperature and absolute magnitude for the different metallicity ranges. Top panel: $[\mathrm{Fe} / \mathrm{H}] \leq-0.5$. Middle panel: $-0.5<[\mathrm{Fe} / \mathrm{H}]<0.0$. Bottom panel: $[\mathrm{Fe} / \mathrm{H}] \geq 0.0$. Black points are simulations and the red line is the fit.

$$
M_{V}\left(T_{\text {eff }}\right)=\left\{\begin{array}{l}
18.80-0.003 T_{\text {eff }}+1.715 \times 10^{-07} T_{\text {eff }}^{2} \\
\text { if }[\mathrm{Fe} / \mathrm{H}] \leq-0.5 \\
26.24-0.006 T_{\text {eff }}+3.821 \times 10^{-07} T_{\text {eff }}^{2} \\
\text { if }-0.5<[\mathrm{Fe} / \mathrm{H}]<0.0 \\
12.39-0.001 T_{\text {eff }} \\
\text { if }[\mathrm{Fe} / \mathrm{H}] \geq 0.0
\end{array}\right.
$$

The V magnitude is computed from the equations (Lupton 2005) ${ }^{1}$ to convert from SDSS to Johnson system. Having the absolute magnitude and the apparent magnitude we can compute the distance modulus for each star. To evaluate the extinction we compute the mean extinction, for each plate (separating bright and faint plates), in the nine different regions already mentioned in the above extinction correction algorithm. Figure 8.6 shows the difference between the new computed distance and the distance given by the model as a function of the distance given by the model for all stars in our sample. There is a clear asymmetry in the y axis for distances larger than $5 \mathrm{kpc}$. This asymmetry is due to the large dispersion for high temperatures in the absolute magnitude temperature relation. This implies that our relation is weaker for high temperatures producing a bias in distances. We have compared the results from our method with the ones

\footnotetext{
${ }^{1}$ http://www.sdss.org/dr4/algorithms/sdssUBVRITransform.html
} 


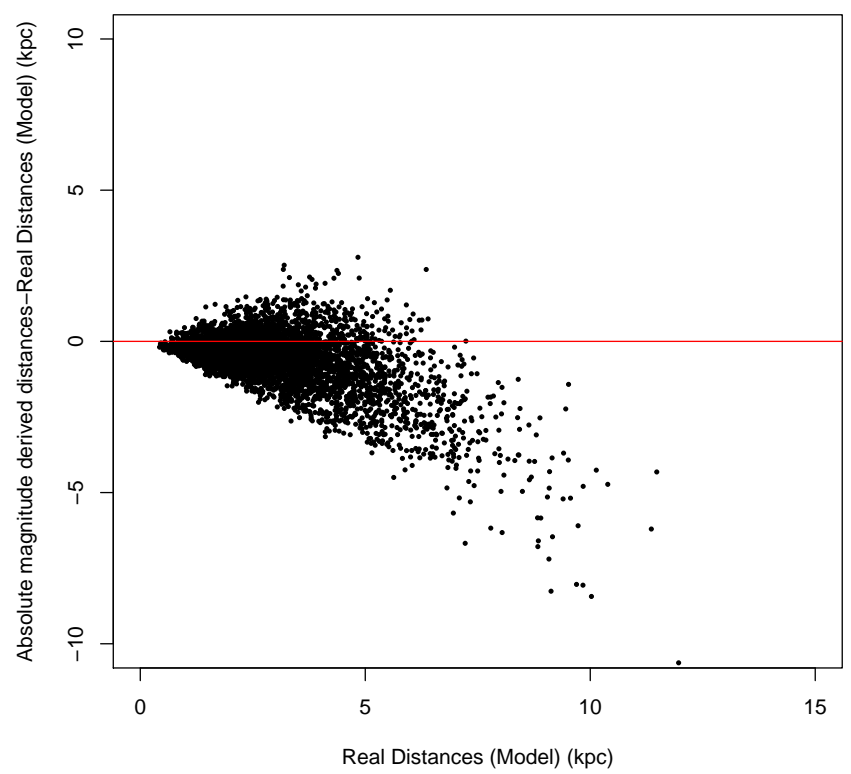

Figure 8.6: Difference between the new computed distance and the distance given by the model as a function of the distance given by the model. The red line is an horizontal line that crosses the $\mathrm{Y}$ axis at zero.

from Ivezić et al. (2008). Figure 8.7 shows the results for the two methods. Ivezić et al. (2008) method computes systematically lower distances, than the ones computed from our method, specially at large distances.

Because we use the same process for observations and simulations, when we proceed with the statistical treatment, in the fitting method, the bias is the same in both catalogs. Therefore our results will be unbiased by the distance computation because we compare two distance distributions having the same bias, avoiding the use of log $\mathrm{g}$ which would add a complex bias to the sample.

\subsubsection{Fitting method}

The objective is to determine the solar neighbourhood (hereafter $\mathrm{SN}$ ) metallicity, the radial metallicity gradient and the dispersion in each population (thin and thick disc populations). It is not possible to compute analytically the likelihood. So, the likelihood we used is an estimate based on the distance between the observations and simulations. The goodness of fit is computed from equation 8.1.5. The data and simulations are binned in the distance estimate (1.0 kpc) metallicity (0.25 dex) space.

We use an ABC/MCMC (Approximate Bayesian Computation) method (Marin et al. 2011), 


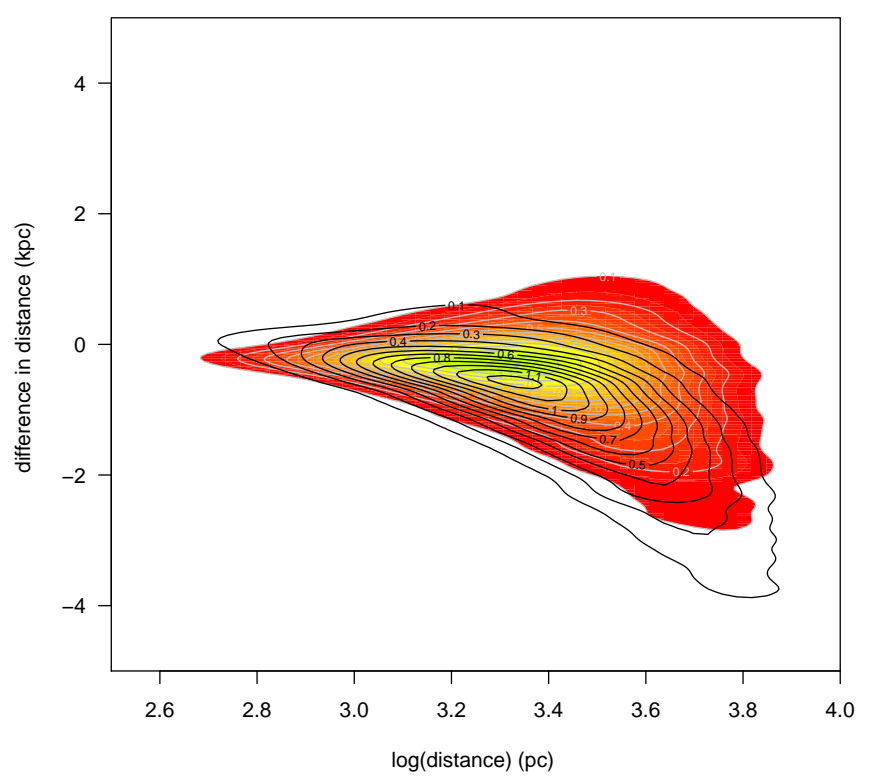

Figure 8.7: Difference between distance estimate and the distance given by the model as a function of the $\log$ (distance) given by the model. Density map and grey contours refer to our method and black contours to Ivezić et al. (2008) method.

where the sampling is done by a Metropolis-Hasting algorithm. The thin disc is composed of 7 different sub-populations of different ages (see section 3.2). We grouped two of these subpopulations in one group, the 'old thin disc', with ages from 5.0 Gyr to $10.0 \mathrm{Gyr}$. We also tried to fit all the subpopulations of the thin disc together. We called this group the 'thin disc'. We also included in the MCMC/ABC method parameters to constrain the vertical metallicity gradient in the thick and thin disc. Unfortunately the low latitude fields do not cover a large range of latitudes so the vertical metallicity gradients are very difficult to constrain.

The range for the parameters is given in Table 8.3. To explore properly the parameter space we perform 10 runs of 10 million iterations each applied on ten different simulations. The errors were computed from the final batch (the final 20\% of the accepted chain) of each Markov chain and by comparison of the different runs. We explored possible correlations between parameters. In the cases where we use different number of parameters we can use the Bayesian information criterion (BIC) (Schwarz 1978) to compare results.

$$
B I C=-2 . \times L_{r}+k \times \ln (n)
$$

where $\mathrm{k}$ is the number of parameters and $\mathrm{n}$ is the number of observations. In our work the 
Table 8.3:

Set of parameter range. The thick disc parameters have the subscript 'Thick', the old thin disc parameters have the subscript 'Old thin' and the thin disc parameters have the subscript 'Thin disc'.

\begin{tabular}{|c|c|c|c|}
\hline Parameter & & Minimum & Maximum \\
\hline$\frac{d[\mathrm{Fe} / \mathrm{H}]}{d R}$ & $(d e x / k p c)$ & -0.15 & 0.15 \\
\hline$[\mathrm{Fe} / \mathrm{H}]_{S N_{\text {Thick }}}$ & $(d e x)$ & -1.0 & -0.2 \\
\hline$(\text { Dispersion })_{\text {Thick }}$ & $(d e x)$ & 0.0 & 0.7 \\
\hline$\frac{d[\mathrm{Fe} / \mathrm{H}]}{d R}$ & $(d e x / k p c)$ & -0.2 & 0.1 \\
\hline$[\mathrm{Fe} / \mathrm{H}]_{S N_{\text {Old Thin }}}$ & (dex) & -0.5 & 0.2 \\
\hline$(\text { Dispersion })_{\text {Old Thin }}$ & $(d e x)$ & 0.0 & 0.6 \\
\hline$\frac{d[\mathrm{Fe} / \mathrm{H}]}{d R}$ & $(d e x / k p c)$ & -0.2 & 0.1 \\
\hline$[\mathrm{Fe} / \mathrm{H}]_{S N_{\text {Thin disc }}}$ & $(d e x)$ & -0.5 & 0.2 \\
\hline$(\text { Dispersion })_{\text {Thin disc }}$ & $(d e x)$ & 0.0 & 0.6 \\
\hline
\end{tabular}

number of observations is $n=5375$.

To allow a more complex variation of metallicities with $R_{g a l}$ we fit separatelly three different cases.

- Case 1: We use all fields in the fit. The sample has 5375 stars.

- Case 2: We do not use the anticenter fields $(2668,2678$ and 2681) in the fit. The sample has 3509 stars.

- Case 3: We do not use the inner fiels (2534 and 2536) in the fit. The sample has 5004 stars.

Case 1 performs a global fit to our sample whereas case 2 and 3 allow an inspection of the metallicity distribution in the inner and outer disc respectively. In the following subsections we will discuss case by case.

\subsubsection{Case 1}

In the general case we fitted the old thin disc and thick disc parameters. Table 8.4 contains the fitted model parameters, along with standard deviation for each parameter. The values are the mean of 10 different likelihood values which allows to check how much the results can change due to different model realisations. These results show that there is a flat gradient for the thick disc $\left(-0.008 \pm 0.015 \mathrm{dex} \mathrm{kpc}^{-1}\right)$ with a SN metallicity of $-0.465 \pm 0.033 \mathrm{dex}$. The correlations between parameters along with the correlation factor are shown in figure 8.8. The figure and the correlation factors show that there is no correlation between the parameters aside the SN 
Table 8.4:

Thick disc and thin disc metallicity mean values, when fitting the thick disc and old thin disc.The SN subscript refers to solar neighbourhood value.

\begin{tabular}{ccccccccc}
\hline \hline case & $\begin{array}{c}{[\mathrm{Fe} / \mathrm{H}]_{S N_{\text {Thick }}}} \\
(\mathrm{dex})\end{array}$ & $\begin{array}{c}\frac{d[\mathrm{Fe} / \mathrm{H}]}{d R} \\
\left(\mathrm{dex} \mathrm{kpc}^{-1}\right)\end{array}$ & $\begin{array}{c}\text { Disp } \\
(\mathrm{dex})\end{array}$ & $\begin{array}{c}{\left[\mathrm{Fe} / \mathrm{H}_{S N_{\text {Old Thin }}}\right.} \\
(\mathrm{dex})\end{array}$ & $\begin{array}{c}\frac{d[\mathrm{Fe} / \mathrm{H}]}{d R} \\
\left(\mathrm{dex} \mathrm{kpc}^{-1}\right)\end{array}$ & $\begin{array}{c}\text { Disp } \\
(\mathrm{dex})\end{array}$ & $\mathcal{L}$ & BIC \\
\hline 1 & -0.465 & -0.008 & 0.319 & -0.116 & -0.079 & 0.135 & -511.05 & \\
& \pm 0.033 & \pm 0.015 & \pm 0.029 & \pm 0.012 & \pm 0.015 & \pm 0.011 & \pm 16.63 & 1084.66 \\
2 & -0.449 & 0.031 & 0.319 & -0.116 & -0.086 & 0.135 & -269.10 & \\
& \pm 0.028 & \pm 0.025 & \pm 0.032 & \pm 0.021 & \pm 0.040 & \pm 0.011 & \pm 9.08 & 587.18 \\
3 & -0.418 & -0.030 & 0.304 & -0.113 & -0.076 & 0.135 & -440.37 & \\
& \pm 0.024 & \pm 0.050 & \pm 0.038 & \pm 0.017 & \pm 0.017 & \pm 0.011 & \pm 16.10 & 931.85 \\
\hline \hline
\end{tabular}

metallicity of the thick disc as a function of the radial metallicity gradient of the thick disc (top right panel in Figure 8.8) and the SN metallicity of the old thin disc as a function of the radial metallicity gradient of the old thin disc (bottom left panel in Figure 8.8).

\subsubsection{Case 2}

Results obtained excluding the anticenter fields $(n=3509)$ are shown in the second group of lines in Table 8.4. The best likelihood is obtained for a SN metallicity of the thick disc of $-0.449 \pm 0.033$ dex and a radial metallicity gradient of this population of $(0.031 \pm 0.025)$ dex $\mathrm{kpc}^{-1}$. The thin disc radial metallicity gradient is found to be $-0.086 \pm 0.040 \mathrm{dex} \mathrm{\textrm {kc } ^ { - 1 }}$ with a $S N$ metallicity of $-0.116 \pm 0.021$ dex. The correlations between parameters along with the correlation factors are shown in Figure 8.9. The gradient of the old thin disc and the SN metallicity of the old thin disc are strongly anticorrelated, the other parameters show almost no correlation. Hence it is difficult to constrain the thin disc radial gradient without anticenter fields with these data.

\section{Considering only the thick disc}

For this case we also considered fitting the thick disc alone and the results are shown in Table 8.5. The best fit is obtained for a SN metallicity of the thick disc of $-0.456 \pm 0.028$ dex and a radial metallicity gradient of this population of $(0.031 \pm 0.025) \mathrm{dex} \mathrm{kpc}{ }^{-1}$, therefore the results are similar, inside errors, to the previous fit. The BIC statistics is similar for both cases.

\section{Fitting the whole thin disc}

Furthermore,for this case we also fit the thick disc along with the whole thin disc (Table 8.6). The results for the thick disc are similar, inside errors, to the previous fits. The results for the thin disc are $-0.087 \pm 0.047 \mathrm{dex} \mathrm{kpc}^{-1}$ with a SN metallicity of $-0.083 \pm 0.030 \mathrm{dex}$. The errors 

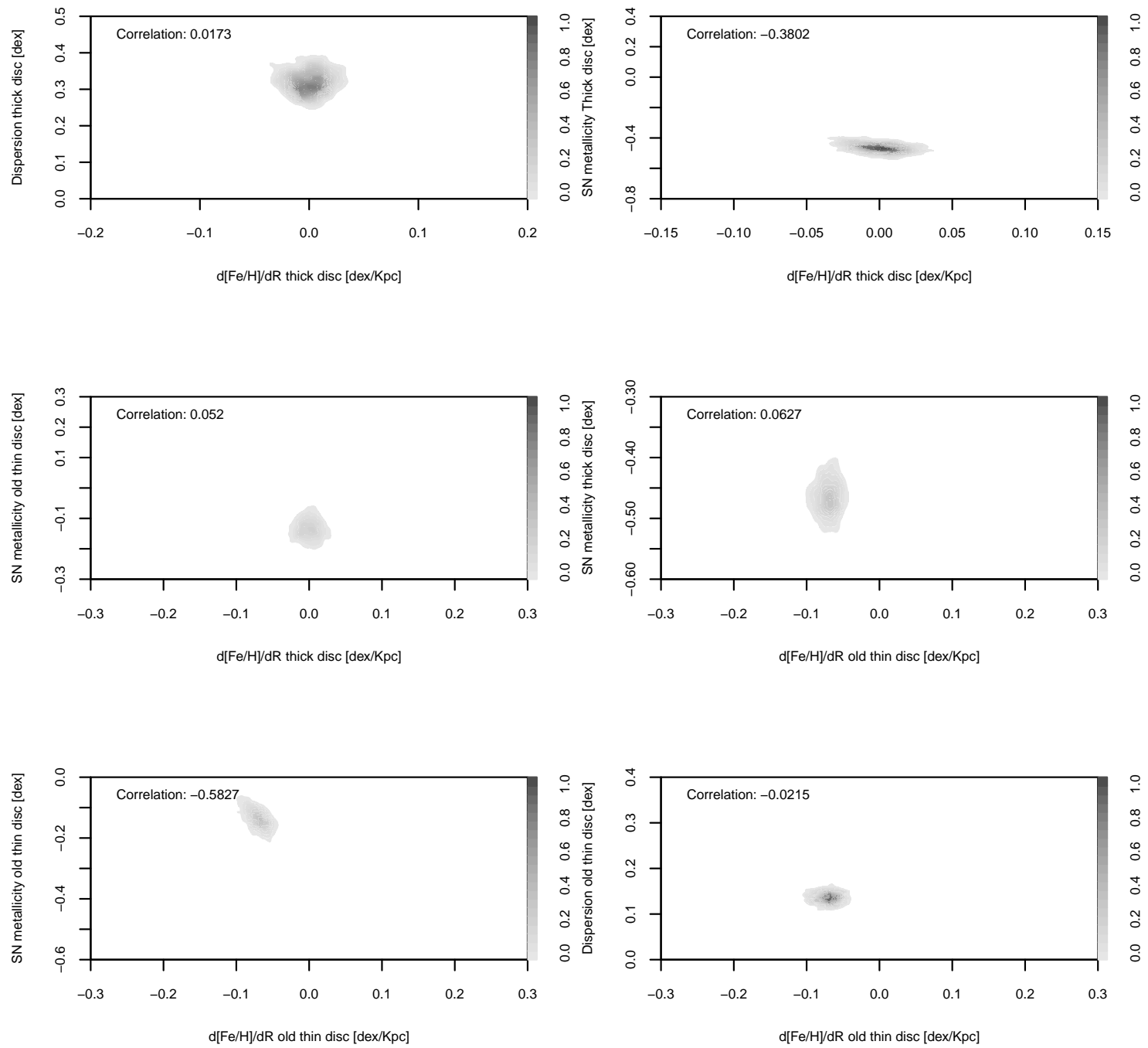

Figure 8.8: Correlations between parameters of the thick disc and old thin disc for case 1. 

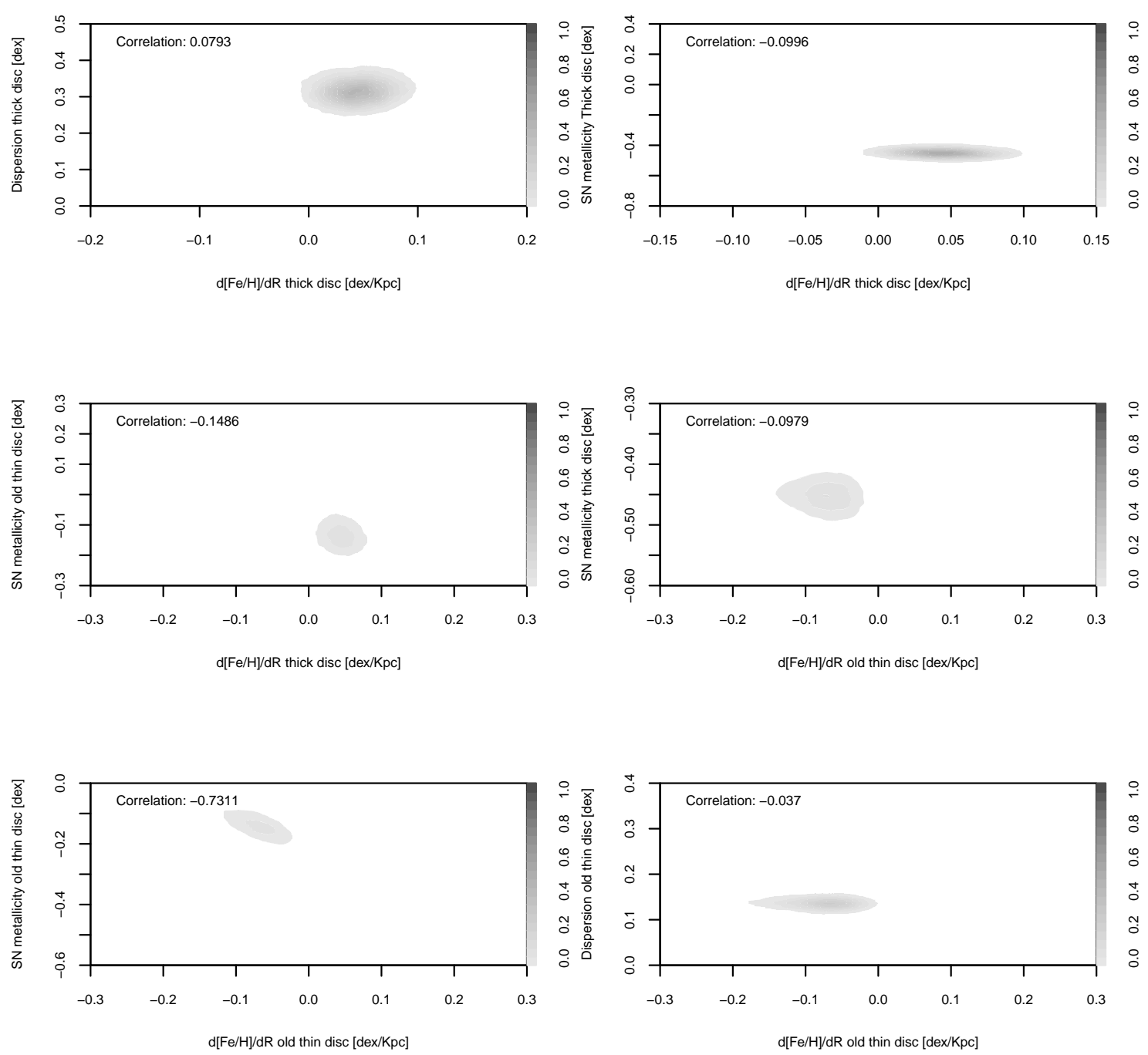

Figure 8.9: Correlations between parameters for the thick disc and old thin disc fitting for case 
Table 8.5:

Thick disc metallicity mean values, when fitting the thick disc alone for case 2

\begin{tabular}{lcccc}
\hline \hline $\begin{array}{l}{[\mathrm{Fe} / \mathrm{H}]_{S N_{\text {Thick }}}} \\
(\mathrm{dex})\end{array}$ & $\begin{array}{c}\frac{d[\mathrm{Fe} / \mathrm{H}]}{d R} \\
\left(\mathrm{dex} \mathrm{kpc}^{-1}\right)\end{array}$ & $\begin{array}{c}\text { Disp } \\
(\mathrm{dex})\end{array}$ & $\mathcal{L}$ & BIC \\
\hline-0.456 & 0.031 & 0.298 & -285.07 & \\
\pm 0.028 & \pm 0.025 & \pm 0.030 & \pm 12.10 & 601.42 \\
\hline \hline
\end{tabular}

Table 8.6:

Thick disc and thin disc metallicity mean set of parameters when fitting the thick disc along with all thin disc - for case 2

\begin{tabular}{lccccccc}
\hline \hline $\begin{array}{l}{[\mathrm{Fe} / \mathrm{H}]_{S N_{\text {Thick }}}} \\
(\mathrm{dex})\end{array}$ & $\begin{array}{c}\frac{d F e / H]}{d R} \\
\left(\mathrm{dex} \mathrm{kpc}^{-1}\right)\end{array}$ & $\begin{array}{c}\text { Disp } \\
(\mathrm{dex})\end{array}$ & $\begin{array}{c}{[\mathrm{Fe} / \mathrm{H}]_{S N_{\text {Thin }}}} \\
(\mathrm{dex})\end{array}$ & $\begin{array}{c}\frac{d[F e / H]}{d R} \\
\left(\mathrm{dex} \mathrm{kpc}^{-1}\right)\end{array}$ & $\begin{array}{c}\text { Disp } \\
(\mathrm{dex})\end{array}$ & $\mathcal{L}$ & $\mathrm{BIC}$ \\
\hline-0.451 & 0.032 & 0.301 & -0.083 & -0.087 & 0.135 & -291.73 & \\
\pm 0.029 & \pm 0.026 & \pm 0.032 & \pm 0.030 & \pm 0.047 & \pm 0.010 & \pm 13.14 & 646.02 \\
\hline \hline
\end{tabular}

for the fit are higher. The likelihood (more negative) and BIC are worst, comparing to the ones from tables 8.4 and 8.5. The young thin disc seems to be unconstrained by these data, probably because the proportion of thin disc stars is too small in these fields (the number of stars in the old thin disc is twice as large compared to the young thin disc). Results for this case point to the possible existence of a positive gradient in the inner thick disc in agreement with Carrell et al. (2012). It will be discussed in section 10.3.1.

\subsubsection{Case 3}

Case 3 exclude inner fields. The results are shown in the third group of lines in Table 8.4. We omit the correlations because they are very similar with the other two cases. There are no correlations between parameters aside radial metallicity gradient of the thin disc as a function of the SN metallicity of the old thin disc which has a correlation factor around -0.6. The old thin disc results are comparable to the ones obtained for the other cases. The SN metallicity increases by one sigma of the previous error $(-0.418 \pm 0.050 \mathrm{dex})$. However we found, for this

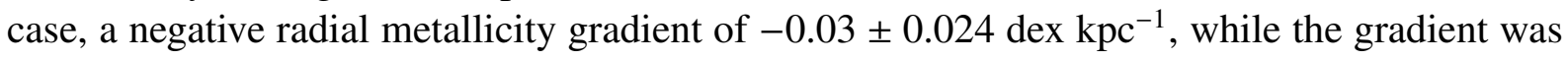
positive when only inner fields were considered (case 2).

The intermediate longitudes have higher metallicity than the anticenter fields and the inner fields. From these data, there is a slight indication that there are two different gradients in the thick disc, one that is positive towards the inner galaxy and one that is negative towards the outer galaxy. Using the same fields (including the anticenter) Cheng et al. (2012b) obtained a flat gradient with a mean metallicity of -0.5 dex. Our results are in agreement with the ones obtained in their work, if we consider all fields together. 

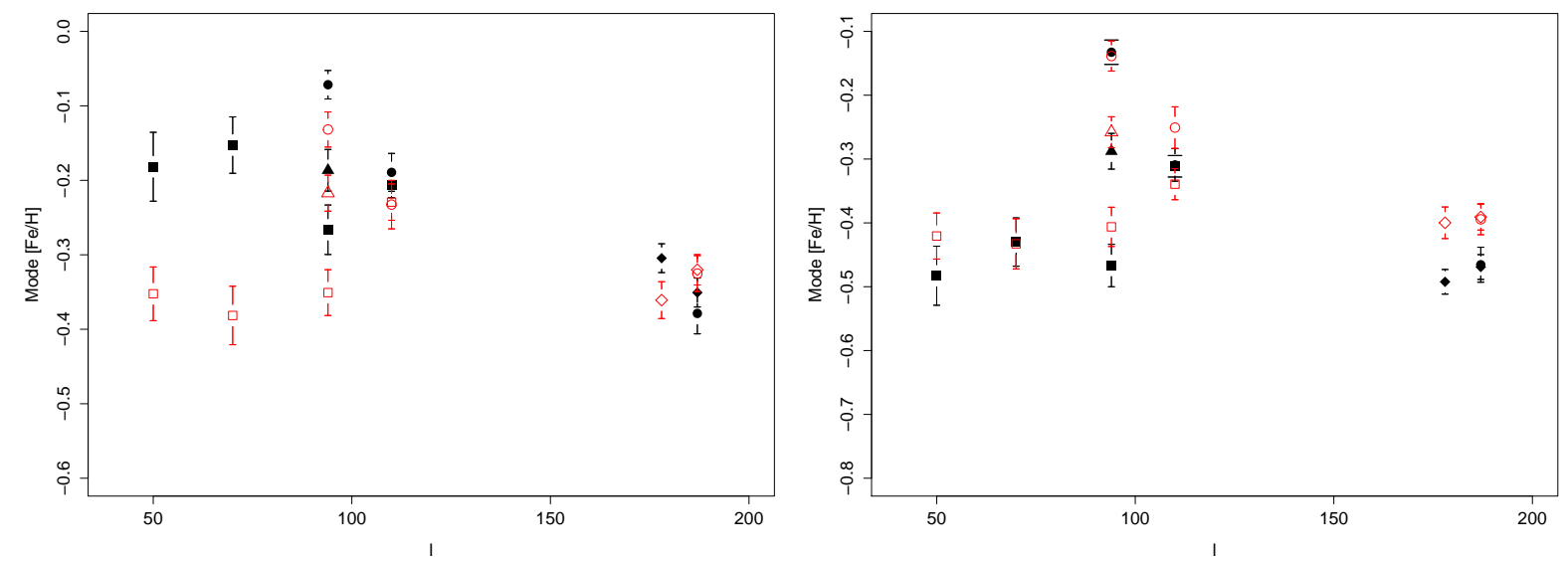

Figure 8.10: Mode of metallicities at different longitudes for the revised model (case 1). Left panel are the bright plates and right panel are the faint plates. The observations are in black and the simulations in red. The standard deviation is represented by the small bars. Squares are latitudes higher or equal to $14^{\circ}$; Circles are latitudes between $8^{\circ}$ and $10.5^{\circ}$; Triangles are latitudes equal to $-8^{\circ}$; Diamonds are latitudes lower than $-8^{\circ}$.

\subsubsection{Revised model}

Simulations were redone using the thick disc results from the table 8.4 and we compared for the different cases the statistics of different astrophysical parameters (metallicity, effective temperature and gravity) of the modified model with the data. We have done 10 simulations and we compute the mean likelihood for each parameter. The sigma is computed from the standard deviation of the 10 runs and is given for each spectroscopic parameter. Results are shown in Table 8.7 for each field. Table 8.8 contains the sum of the $\log$ likelihood in all fields. Figure 8.10 shows the revised model modes for each field as a function of the longitude. In comparison with figure 8.4 the values of the modes of the simulations, are shifted towards higher metallicities, in better agreement with the data but for the bright plates in inner fields the metallicity in the model is still too low.

Comparing the variations in likelihood before and after the fit (Table 8.7 for individual fields and Table 8.8 for all fields together) and for the different fits, we obtain a significant improvement of the model for the metallicity and $\log g$ distribution while the effective temperature distribution is slightly worse, specially for fields with larger longitudes. We show in figure 8.11 the temperature distributions for the original and revised models. There is a clear shift towards lower temperatures from the original to the revised model.

The reason for the disagrement may be due to the isochrone change itself and selection biases from the color selection made to select the MSTO stars. The new isochrone has a higher metallicity which shifts the turnoff position for lower temperatures explaining the lower effective temperature distribution. As we use a different isochrone for the thick disc in the revised 
Table 8.7:

Likelihood values for the spectroscopic parameters with the new sets of parameters

\begin{tabular}{|c|c|c|c|c|c|c|c|c|c|c|}
\hline Fit & Plate & {$[\mathrm{Fe} / \mathrm{H}]$} & $\sigma$ & $\mathrm{T}_{\mathrm{eff}}$ & $\sigma$ & $\log g$ & $\sigma$ & $1(\operatorname{deg})$ & $\mathrm{b}(\operatorname{deg}))$ & Stars \\
\hline Original & 2534 & -90.72 & 17.65 & -43.28 & 11.43 & -55.63 & 14.57 & 50 & 14 & 468 \\
\hline Case 1 & 2534 & -31.12 & 6.97 & -14.11 & 5.40 & -53.74 & 9.25 & & & \\
\hline Case 2 & 2534 & -20.35 & 7.59 & -18.39 & 8.50 & -42.24 & 11.84 & & & \\
\hline Case 3 & 2534 & -31.95 & 6.34 & -16.92 & 6.04 & -50.62 & 14.01 & & & \\
\hline Original & 2536 & -122.71 & 13.85 & -32.64 & 6.77 & -17.82 & 6.15 & 70 & 14 & 497 \\
\hline Case 1 & 2536 & -25.01 & 5.16 & -13.62 & 4.63 & -12.90 & 5.27 & & & \\
\hline Case 2 & 2536 & -26.11 & 9.72 & -13.85 & 5.63 & -13.87 & 2.82 & & & \\
\hline Case 3 & 2536 & -40.72 & 62.79 & -28.69 & 67.76 & -31.41 & 57.76 & & & \\
\hline Original & 2537 & -119.41 & 25.92 & -43.36 & 10.71 & -54.73 & 8.43 & 110 & 10.5 & 494 \\
\hline Case 1 & 2537 & -48.37 & 13.11 & -49.91 & 11.47 & -42.31 & 11.31 & & & \\
\hline Case 2 & 2537 & -45.69 & 10.32 & -54.85 & 7.40 & -46.40 & 9.80 & & & \\
\hline Case 3 & 2537 & -47.35 & 16.95 & -51.34 & 9.40 & -43.16 & 3.94 & & & \\
\hline Original & 2538 & -154.28 & 24.88 & -73.68 & 19.62 & -19.42 & 5.02 & 110 & 16 & 621 \\
\hline Case 1 & 2538 & -50.07 & 11.44 & -111.46 & 25.31 & -17.61 & 5.96 & & & \\
\hline Case 2 & 2538 & -42.21 & 4.66 & -115.96 & 32.84 & -17.13 & 5.45 & & & \\
\hline Case 3 & 2538 & -51.78 & 11.49 & -116.70 & 21.32 & -22.25 & 8.55 & & & \\
\hline Original & 2554 & -177.70 & 16.80 & -14.04 & 4.54 & -45.65 & 11.26 & 94 & 14 & 554 \\
\hline Case 1 & 2554 & -38.77 & 10.92 & -6.27 & 2.73 & -32.92 & 7.59 & & & \\
\hline Case 2 & 2554 & -32.64 & 7.31 & -8.49 & 4.61 & -30.60 & 9.70 & & & \\
\hline Case 3 & 2554 & -41.11 & 12.72 & -8.72 & 2.74 & -29.32 & 11.83 & & & \\
\hline Original & 2555 & -52.99 & 12.82 & -81.73 & 17.72 & -22.22 & 6.19 & 94 & 8 & 381 \\
\hline Case 1 & 2555 & -29.90 & 7.18 & -83.94 & 17.50 & -20.38 & 7.60 & & & \\
\hline Case 2 & 2555 & -22.49 & 7.02 & -91.36 & 21.49 & -20.24 & 6.29 & & & \\
\hline Case 3 & 2555 & -23.19 & 8.81 & -91.76 & 11.65 & -22.89 & 4.76 & & & \\
\hline Original & 2556 & -140.26 & 14.39 & -31.77 & 4.98 & -13.24 & 4.84 & 94 & -8 & 512 \\
\hline Case 1 & 2556 & -54.58 & 11.54 & -44.88 & 7.79 & -8.06 & 3.07 & & & \\
\hline Case 2 & 2556 & -45.44 & 11.70 & -46.89 & 5.86 & -7.27 & 3.68 & & & \\
\hline Case 3 & 2556 & -48.71 & 11.68 & -43.84 & 10.79 & -5.89 & 4.18 & & & \\
\hline Original & 2668 & -182.22 & 20.12 & -145.57 & 17.00 & -84.24 & 16.72 & 187 & -12 & 671 \\
\hline Case 1 & 2668 & -134.63 & 26.26 & -168.22 & 18.84 & -74.16 & 18.66 & & & \\
\hline Case 2 & 2668 & -150.45 & 33.44 & -162.75 & 12.40 & -58.22 & 11.75 & & & \\
\hline Case 3 & 2668 & -138.62 & 15.93 & -169.65 & 14.18 & -72.45 & 20.34 & & & \\
\hline Original & 2678 & -100.43 & 19.95 & -81.50 & 8.50 & -58.61 & 16.39 & 187 & 8 & 555 \\
\hline Case 1 & 2678 & -112.70 & 7.09 & -90.28 & 10.51 & -42.74 & 7.56 & & & \\
\hline Case 2 & 2678 & -128.86 & 29.88 & -82.96 & 17.65 & -48.87 & 11.91 & & & \\
\hline Case 3 & 2678 & -112.74 & 14.35 & -88.81 & 15.77 & -48.44 & 6.15 & & & \\
\hline Original & 2681 & -143.03 & 21.35 & -165.20 & 21.17 & -71.78 & 10.70 & 178 & -15 & 639 \\
\hline Case 1 & 2681 & -80.80 & 17.56 & -207.92 & 30.45 & -56.34 & 14.81 & & & \\
\hline Case 2 & 2681 & -92.72 & 15.39 & -201.74 & 35.19 & -59.73 & 10.84 & & & \\
\hline Case 3 & 2681 & -90.66 & 12.35 & -210.40 & 26.80 & -59.16 & 10.97 & & & \\
\hline
\end{tabular}


Table 8.8:

Sum of the log likelihood values for all fields for the spectroscopic parameters (MSTO stars) with the new set of parameters.

\begin{tabular}{lcccccc}
\hline \hline Fit & {$[\mathrm{Fe} / \mathrm{H}]$} & $\sigma$ & $\mathrm{T}_{\text {eff }}$ & $\sigma$ & $\log g$ & $\sigma$ \\
\hline Original & -1238.75 & 18.77 & -712.76 & 12.46 & -443.33 & 10.03 \\
Case 1 & -605.94 & 11.72 & -790.60 & 13.46 & -361.15 & 9.108 \\
Case 2 & -606.96 & 13.70 & -797.22 & 15.16 & -344.56 & 8.41 \\
Case 3 & -626.82 & 11.50 & -826.82 & 12.29 & -385.58 & 13.09 \\
\hline \hline
\end{tabular}

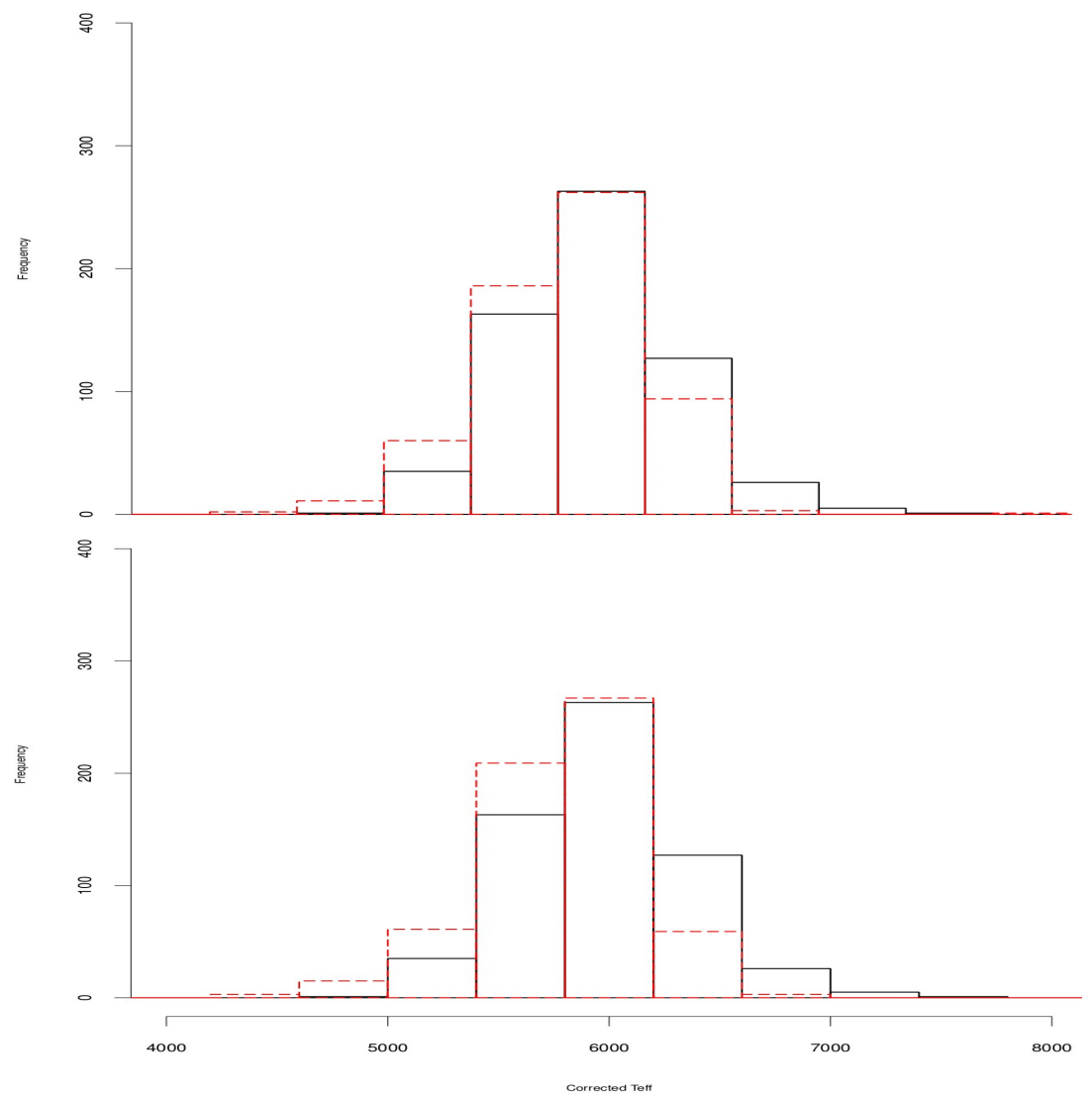

Figure 8.11: Effective temperature distributions for the original model (top panel) and revised model (bottom plot) for plate 2538. Black lines are observations and red lines simulations. 
Table 8.9:

Sum of the likelihood values, for groups of fields of different longitudes, for the spectroscopic parameters (MSTO stars) with the new set of parameters.

\begin{tabular}{lcccccc}
\hline \hline Fit & {$[\mathrm{Fe} / \mathrm{H}]_{g p_{\text {in }}}$} & $\sigma$ & {$[\mathrm{Fe} / \mathrm{H}]_{g p_{\text {no-out }}}$} & $\sigma$ & {$[\mathrm{Fe} / \mathrm{H}]_{g p_{\text {no-in }}}$} & $\sigma$ \\
\hline Case 1 & -56.13 & 6.07 & -277.81 & 9.48 & -549.80 & 13.14 \\
Case 2 & -46.46 & 8.66 & -234.93 & 8.33 & -560.50 & 14.96 \\
Case 3 & -72.67 & 5.34 & -284.80 & 10.33 & -554.15 & 13.03 \\
\hline \hline
\end{tabular}

Table 8.10:

Thick disc and thin disc metallicity mean values, when fitting the thick disc (two slopes) and old thin disc. $R_{\text {change }}$ is the galactocentric radius at which the gradient of the thick disc changes.

\begin{tabular}{|c|c|c|c|c|c|c|c|c|c|}
\hline $\begin{array}{l}{[\mathrm{Fe} / \mathrm{H}]_{S N_{\text {Thick }}}} \\
(\mathrm{dex})\end{array}$ & 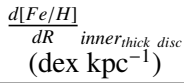 & $\begin{array}{l}\text { Disp } \\
(\operatorname{dex})\end{array}$ & $\begin{array}{c}{[\mathrm{Fe} / \mathrm{H}]_{S N_{\text {oldThin }}}} \\
(\mathrm{dex})\end{array}$ & $\begin{array}{c}\frac{d[F e / H]}{d R} \\
\left(\operatorname{dex~kpc}^{-1}\right)\end{array}$ & $\begin{array}{l}\text { Disp } \\
\text { (dex) }\end{array}$ & $\begin{array}{l}\frac{d[F e / H]}{d R} \text { outer }_{\text {hick }} \text { disc } \\
\left(\operatorname{dex~kpc~}^{-1}\right)\end{array}$ & $\begin{array}{c}R_{\text {change }} \\
\mathrm{kpc}\end{array}$ & $\mathcal{L}$ & BIC \\
\hline-0.453 & 0.007 & 0.318 & -0.121 & -0.079 & 0.135 & -0.032 & 10.12 & -497.70 & 1062.70 \\
\hline \pm 0.036 & \pm 0.033 & \pm 0.031 & \pm 0.017 & \pm 0.017 & \pm 0.011 & \pm 0.046 & \pm 1.33 & \pm 0.51 & \\
\hline
\end{tabular}

model the color distribution of stars is redder because we are considering stars with larger metallicity which changes the extinction correction applied to the simulations. In figure 8.12 we show the total extinction $\left(A_{V}\right)$ as a function of the distance to the Sun and the total extinction $\left(A_{V}\right)$ distribution for the original and revised models. The total extinction applied to the revised version is smaller because, due to the use of the new isochrone, the color distributions is shifted towards red colors. This effect impacts the selection color applied to simulations and consequently the effective temperature distribution.

Table 8.9 shows the likelihoods for the three cases grouped by longitudes. We called $g p_{\text {in }}$ the group of the inner fields (2534 and 2536), $g p_{n o-o u t}$ the group that excludes the anticenter ones and group $g p_{n o-i n}$ excludes the inner fields. $g p_{\text {in }}$ results show that case 2 gives the best likelihood as for the $g p_{n o-o u t}$ confirming the results for case 2 from the MCMC analysis. The $g p_{n o-i n}$ results shows that case 1 and case 2 are compatible inside errors when we fit without the inner fields. This results confirm the possible existence of a positive gradient in the inner disc. For $g p_{n o-i n}$, in all cases the likelihood is similar within error, which means that these fields alone are not sensitive enough to the model parameters.

\subsubsection{Fitting two slopes in the thick disc}

To analyse and test the results obtained for the metallicity distribution and the possible inversion of the metallicity gradient we tried to fit one metallicity distribution associated with a inner and a outer thick disc gradient using all fields as in case 1 incorporating a new parameter $R_{\text {change }}$ which controls the galactocentric radius at which the gradient changes. Along with this parameters we fit also the old thin disc. Table 8.10 shows the results for the fit of the two gradients. 

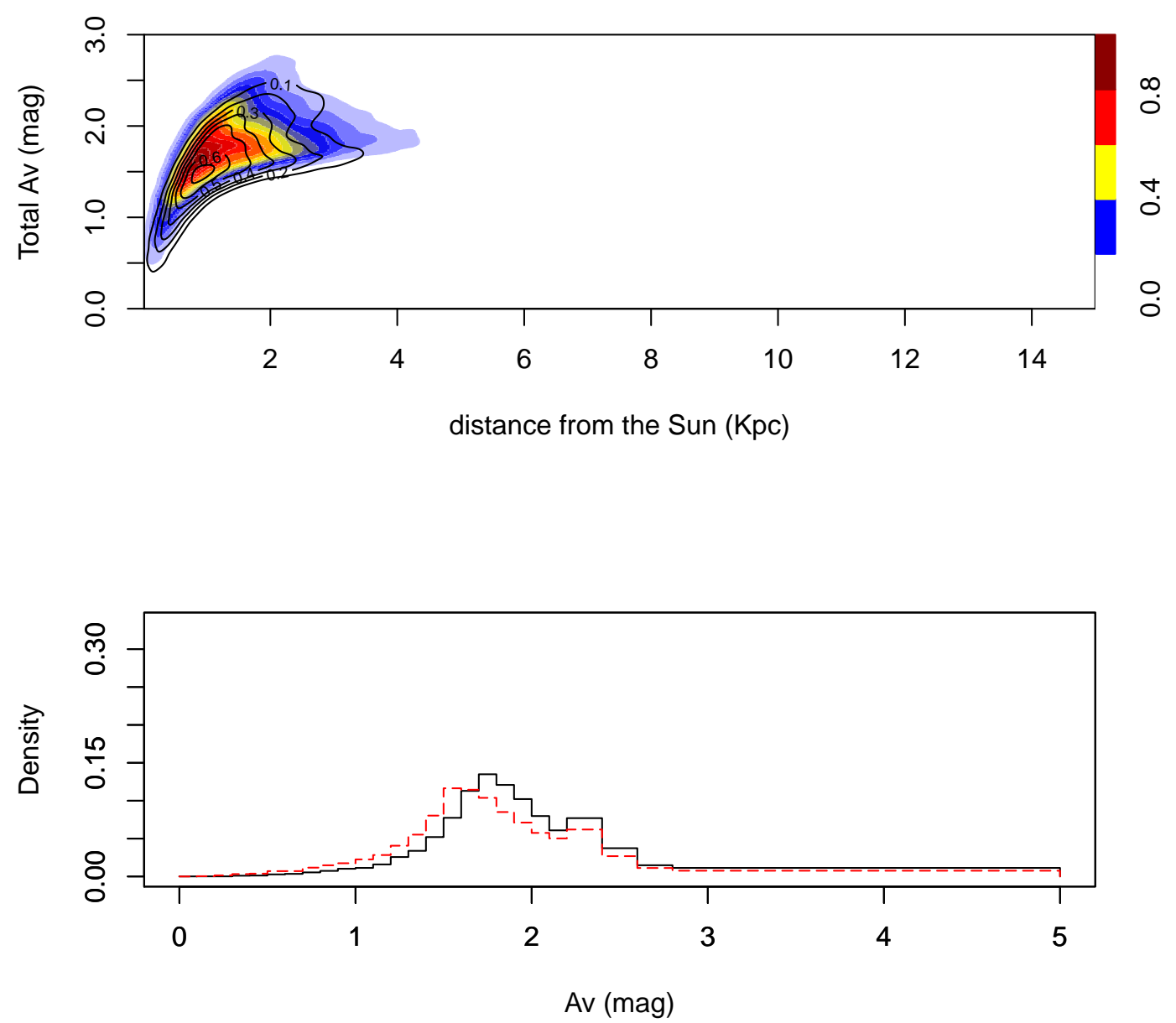

Figure 8.12: Top panel: total extinction applied to the simulations as a function of the distance to the Sun. Density plot refers to the original simulation and black contours to the revised version. Bottom panel: Total extinction distribution applied to the original model (black lines) and to the revised model (red lines). 
Table 8.11:

Sum of the likelihood values, for different ages of the thick disc, for the spectroscopic parameters (MSTO stars) with the fitted parameters.

\begin{tabular}{lcccccc}
\hline \hline Age & {$[\mathrm{Fe} / \mathrm{H}]$} & $\sigma$ & $\mathrm{T}_{\text {eff }}$ & $\sigma$ & $\log g$ & $\sigma$ \\
\hline $8 \mathrm{Gyr}$ & -734.31 & 23.34 & -1133.74 & 49.38 & -424.284 & 17.06 \\
$9 \mathrm{Gyr}$ & -693.68 & 10.78 & -1032.26 & 10.84 & -400.93 & 5.780 \\
$10 \mathrm{Gyr}$ & -695.19 & 15.90 & -990.16 & 39.27 & -449.12 & 14.49 \\
$11 \mathrm{Gyr}$ & -695.84 & 10.09 & -969.23 & 10.22 & -388.55 & 6.63 \\
$12 \mathrm{Gyr}$ & -605.94 & 11.72 & -790.60 & 13.46 & -361.15 & 9.108 \\
$13 \mathrm{Gyr}$ & -706.82 & 13.56 & -1025.64 & 31.84 & -448.57 & 13.79 \\
\hline \hline
\end{tabular}

The table shows that the SN metallicity and dispersion in the thick disc and the SN metallicity and dispersion of the thin disc are well in agreement with the results of table 8.4. The gradient changes at around $10.1 \pm 1.3$ beyond the solar position. The gradient of the outer regions of thick disc is compatible with case 3 of table 8.4 and the best fit for the inner regions is no gradient. The BIC values are inside errors compatible with case 1 of table 8.4. This result reinforces the existence of no gradient in the thick disc. Regarding this result the formation of thick disc may be a combination of the scenarios described in section 10.3.

\subsubsection{The age of the thick disc}

In order to explore the capability of these data to constrain the age of the thick disc we tried different ages for the thick disc isochrones. We use case 1 as the most robust fit. From earlier studies, we assume the range of ages to test to be from $8 \mathrm{Gyr}$ to $13 \mathrm{Gyr}$. We have fitted the simulations, for each age, and the metallicity parameters that we obtain are similar to the ones presented for case 1 (i.e. a SN metallicity of $\sim-0.5$ dex and no radial gradient). We present in Table 8.11 the results taking the mean of the likelihoods in five different simulations. The bright and faint plates are treated together. From these results, assuming that the thick disc has a single epoch formation, we can say that there is an indication that $12 \mathrm{Gyr}$ is the best age for this population or at least that the isochrone from Bergbush and vandenberg (1992) that best fits these data is the one with $\mathrm{Fe} / \mathrm{H}=-0.5$ and age of $12 \mathrm{Gyr}$.

\subsection{The metallicity distribution in the model $B$ revised ver- sion}

To test whether the results depends on the population synthesis hypothesis, we used the revised version model $\mathrm{B}$ of the BGM described in section 3.4 to constrain the gradients and metallicity distributions with the MCMC analysis. The results for case 1 and case 2 shown in table 8.12 
Table 8.12:

Thick disc and thin disc metallicity mean set of parameters - Fitting the old thin disc for the model B of the revised version.

\begin{tabular}{lcccccccc}
\hline \hline $\begin{array}{l}\text { case } \\
(\mathrm{dex})\end{array}$ & $\begin{array}{c}{[\mathrm{Fe} / \mathrm{H}]_{S N_{\text {Thick }}}} \\
\left.(\mathrm{dex} \mathrm{kpc})^{-1}\right)\end{array}$ & $\begin{array}{c}\frac{d[F e / H]}{d R} \\
(\mathrm{dex})\end{array}$ & $\begin{array}{c}\text { Disp } \\
(\mathrm{dex})\end{array}$ & $\begin{array}{c}{[\mathrm{Fe} / \mathrm{H}]_{S_{\text {OUlThin }}}} \\
\left(\mathrm{dex} \mathrm{kpc}^{-1}\right)\end{array}$ & $\begin{array}{c}\frac{d F e / H]}{d R} \\
(\mathrm{dex})\end{array}$ & $\begin{array}{c}\text { Disp } \\
\left(\mathrm{dex} \mathrm{kpc}^{-1}\right)\end{array}$ & $\begin{array}{c}\mathcal{L} \\
\mathrm{kpc}\end{array}$ & $\mathrm{BIC}$ \\
\hline 1 & -0.452 & 0.006 & 0.333 & -0.119 & -0.083 & 0.137 & -527.84 & 1107.21 \\
& \pm 0.034 & \pm 0.022 & \pm 0.029 & \pm 0.018 & \pm 0.021 & \pm 0.027 & & \\
2 & -0.431 & 0.030 & 0.347 & -0.119 & -0.086 & 0.135 & -305.72 & 662.97 \\
& \pm 0.028 & \pm 0.025 & \pm 0.029 & \pm 0.021 & \pm 0.024 & \pm 0.031 & & \\
\hline \hline
\end{tabular}

are the mean and dispersion of $10 \mathrm{MCMC}$ runs for one simulation. Inside errors results are in agreement with case 1 and case 2 for the original model presented in table 8.4 for all parameters. The likelihood and BIC are slightly higher than with the original model.

\subsection{K giants sample}

In figures 8.13 and 8.14 we compare observations and simulations for the $\mathrm{K}$ giants selection in plate 2536 and 2544 respectively. The effective temperatures have been corrected and stars with effective temperatures larger than $4900 \mathrm{k}$ have been selected as explained in section 5.2. The log $\mathrm{g}$ distributions, for both plates, are peaked at $\sim 4.5$ with a weak tail towards the giants, which means that the $\mathrm{K}$ giants selection is too polluted by other stars. For plate 2536 there is a good agreement between the observations and simulations for the metallicity and log g distributions. The effective temperature distribution is too wide in the simulations and the peak is shifted towards larger temperatures. For plate 2544 the metallicity distribution in the simulations is lower than the one from observations and the effective temperature is shifted. Since the K giant sample is dominated by main sequence stars we have decided not to pursue its analysis.

\subsection{Comparison with previous works}

\subsubsection{Thin disc}

Chemical gradients in both radial and vertical directions have been studied using different tracers that have different ages. In open clusters metallicity and distances are well determined which makes these objects good tracers to analyze chemical space distributions (e.g., Carrera \& Pancino 2011; Friel et al. 2002; Frinchaboy et al. 2013). Several works have been using young objects like HII regions (e.g., Rudolph et al. 2006; Quireza et al. 2006) or B stars (Daflon et al. 2010). Older populations like red giants (Hayden et al. 2014)) and Planetary nebulae (e.g., Henry et al. 2010; Stanghellini et al. 2006) have been studied. Nevertheless, cepheid variables (e.g., Luck et al. 2011; Lemasle et al. 2013) remain the most accurate indicator, of present day abundance gradients in the Milky Way, due to the large distances at which they can 

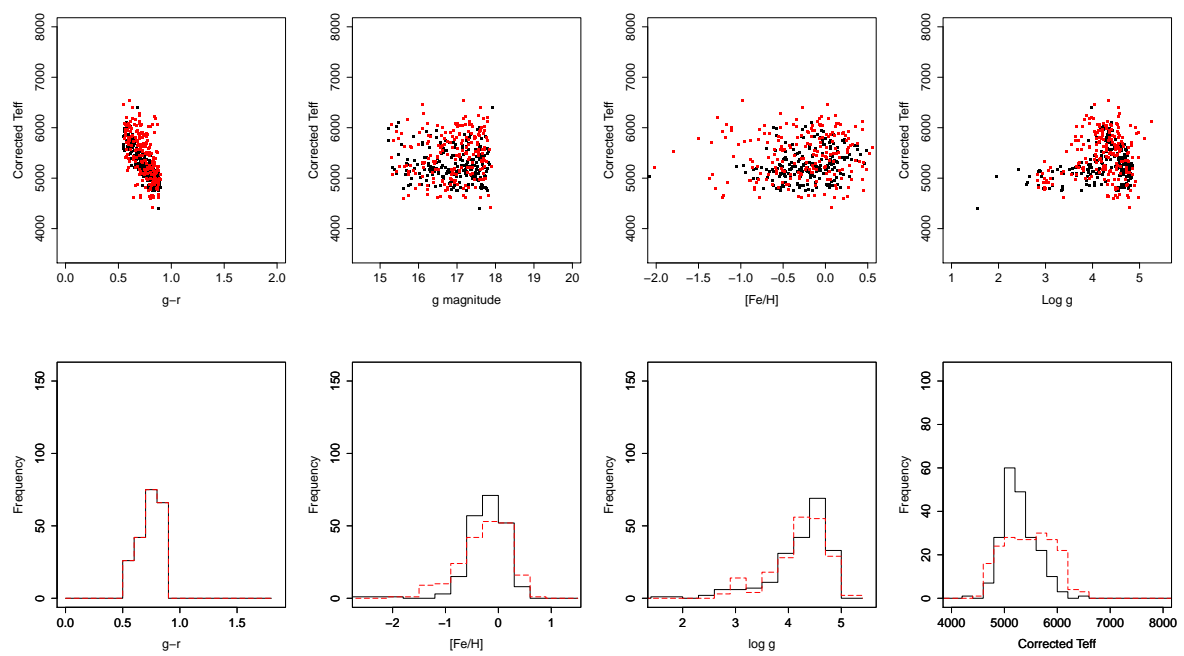

Figure 8.13: Comparison of the spectrocopic observations and simulations for the bright plate 2536.
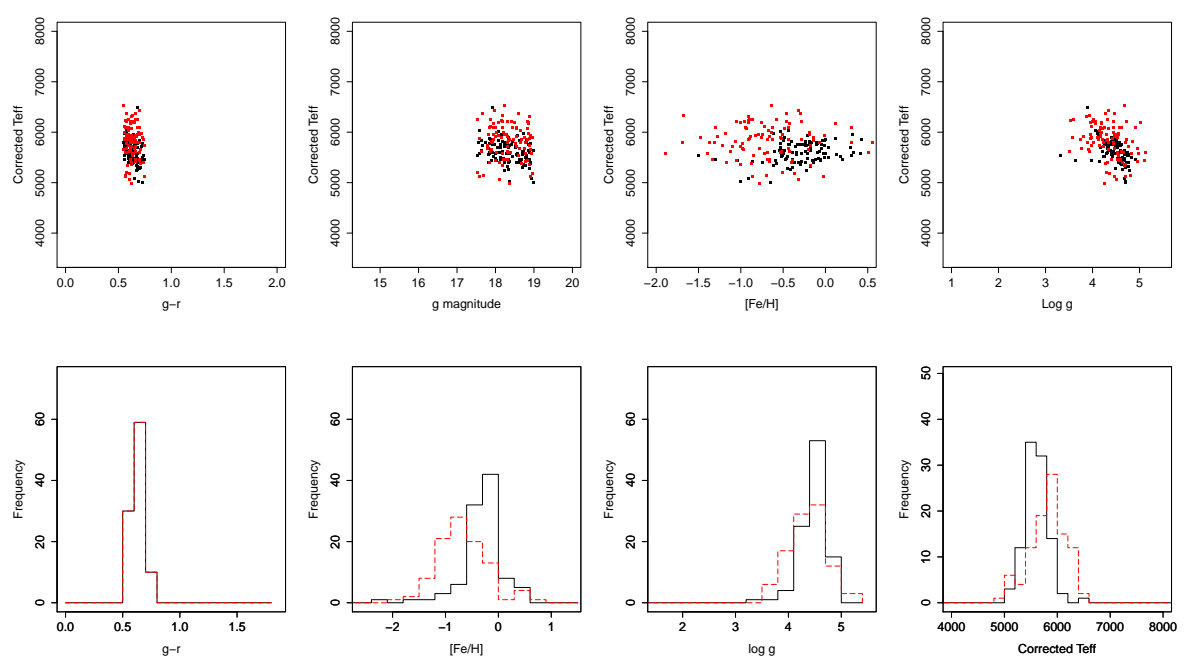

Figure 8.14: Comparison of the spectrocopic observations and simulations for the faint plate 2544. 
Table 8.13:

Thin disc metallicity distribution from the literature

\begin{tabular}{|c|c|c|c|}
\hline Author & $\begin{array}{c}\overline{[\mathrm{Fe} / \mathrm{H}]} \\
\operatorname{dex}\end{array}$ & $\begin{array}{c}\frac{d[\mathrm{Fe} / \mathrm{H}]}{d R} \\
\operatorname{dex} \mathrm{kpc}^{-1}\end{array}$ & Comments \\
\hline Friel et al. (2002) & & -0.06 & Open clusters \\
\hline Soubiran et al. (2003) & $-0.17 \pm 0.01$ & & HR Red clump giants \\
\hline Nordström et al. (2004) & $-0.19 \pm 0.20$ & -0.099 & $\mathrm{~F}$ and $\mathrm{G}$ dwarf stars - GCS \\
\hline Lee et al. (2011) & $\sim-0.2$ & -0.09 & G dwarfs - SEGUE \\
\hline Fuhrmann (2011) & $-0.034 \pm 0.015$ & & 300 nearby solar-type stars \\
\hline Cheng et al. (2012b) & $\sim-0.2$ & $-0.066 \pm 0.044$ & MSTO stars - SEGUE \\
\hline Boeche et al. (2013) & & $-0.065 \pm 0.002$ & Rave dwarf stars \\
\hline Frinchaboy et al. (2013) & & $\begin{array}{c}-0.09 \pm 0.03 \text { for all sample } \\
-0.20 \pm 0.08 \rightarrow 8.0<R_{\text {gal }}<10.0 \mathrm{kpc} \\
-0.02 \pm 0.09 \rightarrow R_{\text {gal }}>10.0 \mathrm{kpc}\end{array}$ & Open clusters - APOGEE \\
\hline Hayden et al. (2014) & $0.02 \pm 0.02$ & $-0.09 \pm 0.02$ & Red giants - APOGEE \\
\hline
\end{tabular}

be found and to their high intrinsic luminosity which allows to measure accurate spectral parameters and distances (See Maciel \& Costa (2010) for a review). Recent spetroscopic surveys (GCS, 2MASS, SDSS, RAVE, GES among others) gave new constrains of metallicity gradients. (e.g., Nordström et al. 2004; Kordopatis et al. 2013b; Recio-Blanco et al. 2014; Anders et al. 2014; Mikolaitis et al. 2014). Table 8.13 summarizes most recent results from literature for the metallicity distribution of the thin disc.

We obtain for the old thin disc a SN metallicity around $-0.116 \pm 0.021$ dex. Our results, inside errors, are in agreement with the literature. In comparison with Cheng et al. (2012b) lower $|z|$ slice we obtain a slightly higher SN metallicity. The discrepancy is probably due to the fact that they compute the mean metallicity for a slice in $|z|$ and not for a pure thin disc population as we do.

A radial gradient around $-0.086 \pm 0.04 \mathrm{dex} \mathrm{kpc}^{-1}$ is obtained for the old thin disc in our study. The results obtained from this work, inside errors, are in agreement with other works.

\subsubsection{Thick disc}

The results presented in Table 8.4 indicate a SN metallicity around $-0.465 \pm 0.033$ dex in agreement with most of the recent works presented in Table 8.14. Few studies obtained different results (e.g., Gilmore et al. 1995; Nordström et al. 2004) because they use different sample and they use different means to distinguish the thick from the thin disc and the halo (i.e. they slice in $|z|$ to select the population) or they use kinematics..

Our null gradient for the thick disc $\left(-0.008 \pm 0.015 \mathrm{dex} \mathrm{kpc}^{-1}\right)$ is in agreement with Cheng et al. (2012b) who obtained $0.0028 \pm 0.0071 \mathrm{dex} \mathrm{kpc}^{-1}$, Hayden et al. (2014) $(-0.003 \pm 0.006 \mathrm{dex}$ $\mathrm{kpc}^{-1}$ ) which used a sample of red giants from APOGEE, Boeche et al. (2013) (0.006 0.015 
Table 8.14:

Thick disc metallicity distribution from the literature

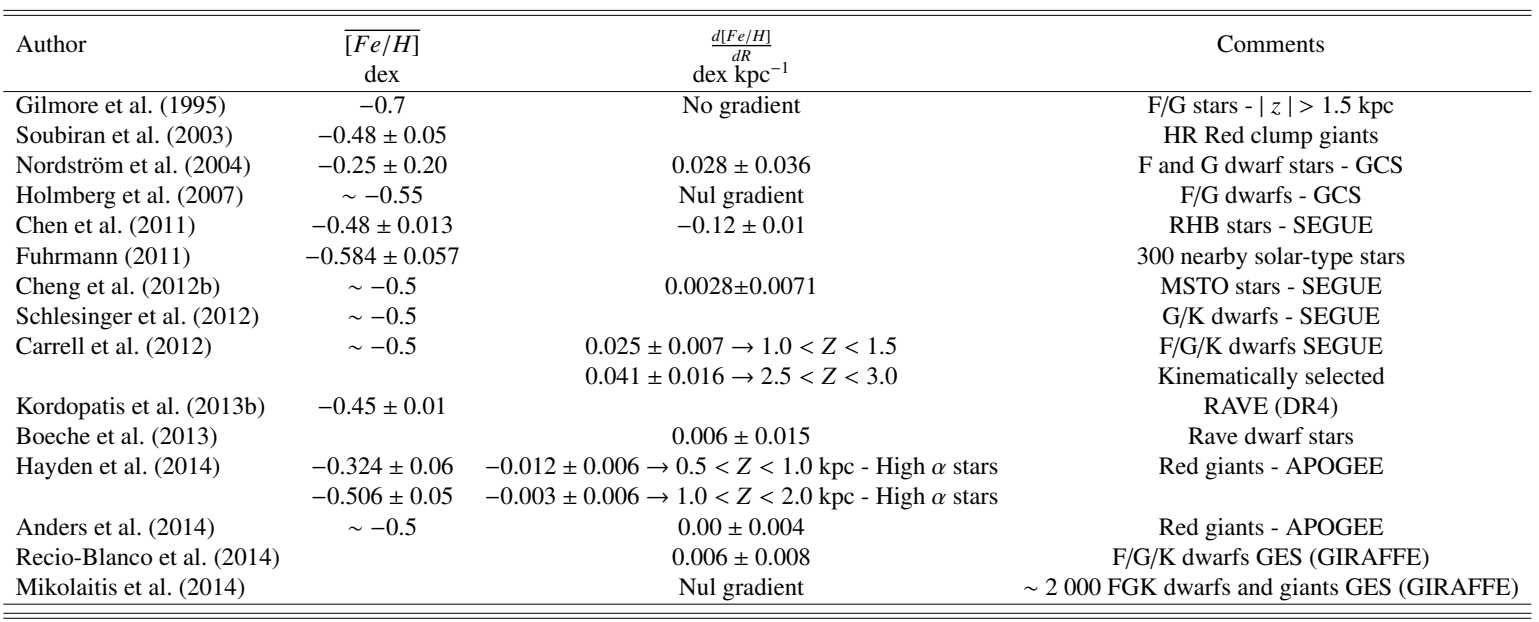

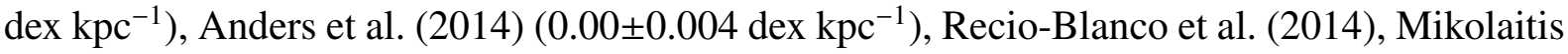
et al. (2014). These works agree about the flattening of the radial metallicity gradient at higher distances from the plane. However these results are in disagreement with Chen et al. (2011) that argued for the existence of a strong negative gradient $\left(-0.12 \pm 0.01 \mathrm{dex} \mathrm{kpc}^{-1}\right)$ in the thick disc from their sample of RHB stars. On the contrary Carrell et al. (2012) found a positive gradient for the thick disc with their kinematically selected sample (in section 10.3.1 we discuss in more detail this result). 


\section{Chapter 9}

\section{Spectroscopic results from the GES data}

For this analysis we use the BGM version which accounts for a longer star formation period during the thick disc phase described in section 3.5. In the following sections we describe the method and analysis done comparing simulations from this version of the BGM with GES data.

\subsection{Photometric errors}

The photometric system given by the BGM is the one used in 2MASS survey so we have used the transformations given by Soto et al. (2013) to convert magnitudes and colors for the VVV photometric system. To simulate the photometric errors in the simulations we have fitted the photometric error as a function of the magnitude for bands $\mathrm{J}, \mathrm{H}$ and $\mathrm{K}$ using an exponential law Error $_{\text {mag }} g_{J K}=a+\exp \left(-b+c * m a g_{J H K}\right)$ for few fields and tooked the mean of each fitted parameter. VISTA magnitudes are given two decimal precisions. Therefore the a parameter is at least 0.01 mags (Private communication from Saito). Table 9.1 shows the mean results. We have included the photometric errors in the simulation by drawing errors from a Gaussian distribution.

Table 9.1:

The value of the parameters used to compute the photometric errors in the simulation

\begin{tabular}{lccc}
\hline \hline Band & $\mathrm{a}$ & $\mathrm{b}$ & $\mathrm{c}$ \\
\hline $\mathrm{J}$ & 0.01 & 21.11 & 0.984 \\
$\mathrm{H}$ & 0.01 & 20.34 & 0.986 \\
$\mathrm{~K}$ & 0.01 & 19.58 & 0.983 \\
\hline \hline
\end{tabular}




\subsection{Extinction}

To compare model with observations we have to apply an extinction model to the simulations from the BGM. In order to test if the extinction from Schlegel extinction maps (Schlegel et al. 1998) can be used for the GES disc fields we have computed the mean reddening for each of the observed disc fields in Gaia-ESO Survey. Table 9.2 shows the results. From the table we see that the GES disc fields suffer from low reddening in average $(\sim 0.05)$ but there are some fields were the reddening is higher (fields with reddening larger than 0.25 are in bold).

Table 9.2:

Mean reddening for each of the observed disc fields in GES

\begin{tabular}{lccc}
\hline \hline Field & $\begin{array}{c}\text { 1 } \\
\left.{ }^{\circ}\right)\end{array}$ & $\begin{array}{c}\mathrm{b} \\
\left(^{\circ}\right)\end{array}$ & $\begin{array}{c}\text { Mean E(B-V) } \\
(\mathrm{mag})\end{array}$ \\
\hline 000400010000 & 97.4333 & -61.5059 & 0.0335 \\
000400470000 & 324.9635 & -68.0406 & 0.0105 \\
002000010000 & 105.5221 & -62.7944 & 0.0367 \\
002000470000 & 318.1591 & -69.1909 & 0.0114 \\
002959033000 & 109.7612 & -65.8181 & 0.0427 \\
002959500600 & 311.6242 & -66.6682 & 0.0117 \\
003240440056 & 314.3819 & -72.6887 & 0.0075 \\
004000010000 & 116.4808 & -63.7293 & 0.0227 \\
004000470000 & 308.6863 & -70.0057 & 0.0113 \\
005000033000 & 122.0503 & -66.3708 & 0.0466 \\
005000500600 & 303.5252 & -67.0218 & 0.012 \\
005959010000 & 127.7875 & -63.7933 & 0.0376 \\
005959470000 & 298.6443 & -70.056 & 0.0131 \\
011959010000 & 138.7815 & -62.9931 & 0.0394 \\
011959470000 & 289.0232 & -69.3523 & 0.0098 \\
014000010000 & 148.9815 & -61.3958 & 0.0308 \\
014000470000 & 280.4699 & -67.9542 & 0.0153 \\
015959010000 & 157.9989 & -59.1308 & 0.0266 \\
015959470000 & 273.2971 & -66.0007 & 0.0205 \\
022000470000 & 267.6228 & -63.5858 & 0.02 \\
023000033000 & 172.1166 & -56.6476 & 0.0288 \\
023959010000 & 172.3794 & -53.1104 & 0.031 \\
023959470000 & 263.2037 & -60.8926 & 0.0186 \\
025556002846 & 176.4049 & -49.9664 & 0.0678 \\
025559003000 & 176.4435 & -49.9749 & 0.0677 \\
030000470000 & 259.8427 & -57.9307 & 0.0129 \\
030959500600 & 263.4263 & -55.0372 & 0.0212 \\
& & & \\
& &
\end{tabular}


continued from previous page

\begin{tabular}{lccc}
\hline \hline Field & l & $\mathrm{b}$ & Mean E(B-V) \\
& $\left(^{\circ}\right)$ & $\left(^{\circ}\right)$ & \\
\hline 031800003000 & 181.9725 & -45.8948 & 0.0651 \\
032000470000 & 257.3047 & -54.8174 & 0.0144 \\
033000500600 & 261.1165 & -52.1336 & 0.0118 \\
033800273000 & 223.3754 & -53.1838 & 0.0117 \\
033959000000 & 186.1927 & -41.2921 & 0.0878 \\
033959470000 & 255.4938 & -51.5834 & 0.0087 \\
035959470000 & 254.261 & -48.2724 & 0.0092 \\
041000500600 & 258.2034 & -46.014 & 0.0159 \\
041959001959 & 193.7336 & -33.2971 & 0.0901 \\
043000500600 & 257.4094 & -42.8559 & 0.0116 \\
044000500600 & 257.1272 & -41.2643 & 0.0133 \\
050000520000 & 259.2574 & -37.9788 & 0.011 \\
051959540000 & 261.598 & -34.8999 & 0.0252 \\
070359423000 & 253.021 & -15.7917 & 0.1032 \\
072048003000 & 216.7397 & 6.3021 & 0.1374 \\
$\mathbf{0 7 4 5 0 0 4 2 3 0 0 0}$ & $\mathbf{2 5 6 . 3 6 8 6}$ & $\mathbf{- 8 . 9 8 2}$ & $\mathbf{0 . 3 6 8 3}$ \\
075600090000 & 228.5067 & 9.964 & 0.0803 \\
075959003000 & 221.3949 & 14.967 & 0.0325 \\
082312052959 & 228.8929 & 17.5586 & 0.0434 \\
083959003000 & 226.56 & 23.691 & 0.032 \\
092758003043 & 233.7991 & 33.8884 & 0.0311 \\
092800003000 & 233.8015 & 33.9044 & 0.031 \\
094753102657 & 246.7813 & 31.7726 & 0.0447 \\
095958410150 & 271.3995 & 11.1151 & 0.2059 \\
100000410000 & 271.3826 & 11.1412 & 0.2043 \\
100759080000 & 248.6133 & 37.0959 & 0.0431 \\
100913412801 & 273.084 & 11.8159 & 0.177 \\
101428405235 & 273.5493 & 12.8694 & 0.175 \\
102800410000 & 275.8022 & 14.1972 & 0.1264 \\
103959410000 & 277.7933 & 15.3714 & 0.0856 \\
105731124726 & 264.5839 & 41.4514 & 0.0363 \\
105808154324 & 266.8911 & 39.0943 & 0.0577 \\
105959410000 & 281.2715 & 17.1368 & 0.1261 \\
110009410232 & 281.3219 & 17.1128 & 0.1281 \\
110053132816 & 266.0057 & 41.3453 & 0.0383 \\
112200100000 & 269.6091 & 47.0036 & 0.0492 \\
112800410000 & 286.399 & 19.1806 & 0.1306 \\
113159435959 & 288.158 & 16.5758 & 0.084 \\
120000090000 & 282.1134 & 51.7844 & 0.0447 \\
120000410000 & 292.5759 & 20.8361 & 0.1492 \\
121159410000 & 294.9616 & 21.2586 & 0.1254
\end{tabular}


continued from previous page

\begin{tabular}{lccc}
\hline \hline Field & 1 & $\mathrm{~b}$ & Mean E(B-V) \\
& $\left(^{\circ}\right)$ & $\left(^{\circ}\right)$ & \\
\hline 122759410000 & 298.1712 & 21.6541 & 0.0882 \\
124224130559 & 299.5267 & 49.7121 & 0.0509 \\
124359060000 & 299.536 & 56.8251 & 0.0257 \\
124359090000 & 299.8108 & 53.8198 & 0.0346 \\
124359410000 & 301.4142 & 21.8501 & 0.1415 \\
125609451238 & 303.8018 & 17.6518 & 0.1023 \\
130047410000 & 304.8281 & 21.8387 & 0.1223 \\
131359410000 & 307.508 & 21.6702 & 0.1002 \\
131359460007 & 307.0158 & 16.6899 & 0.1143 \\
132000050000 & 316.1287 & 57.1295 & 0.0397 \\
132000090000 & 314.7758 & 53.2216 & 0.037 \\
132000130000 & 313.6272 & 49.2724 & 0.0508 \\
132800410000 & 310.3277 & 21.3475 & 0.1029 \\
133026434759 & 310.3363 & 18.5143 & 0.1213 \\
134400410000 & 313.5079 & 20.7907 & 0.0842 \\
140000100000 & 329.3628 & 49.3221 & 0.0435 \\
140000410000 & 316.6275 & 20.0391 & 0.0783 \\
140000450000 & 315.4695 & 16.1944 & 0.0874 \\
142000050000 & 339.9213 & 51.3929 & 0.0462 \\
142000410000 & 320.4204 & 18.8484 & 0.1064 \\
142145440827 & 319.5711 & 15.7946 & 0.1162 \\
142800090000 & 339.2173 & 46.9602 & 0.0576 \\
143959410000 & 324.0767 & 17.3729 & 0.0967 \\
144113400831 & 324.68 & 18.0482 & 0.0914 \\
145800410000 & 327.2323 & 15.8234 & 0.0953 \\
150159100000 & 347.8423 & 41.2106 & 0.0964 \\
150512400000 & 328.9853 & 16.0101 & 0.0904 \\
151114404800 & 329.5627 & 14.7365 & 0.0951 \\
151712413000 & 330.1636 & 13.5388 & 0.1336 \\
152311421200 & 330.7191 & 12.3387 & 0.1509 \\
152936425359 & 331.3066 & 11.0943 & 0.1701 \\
$\mathbf{1 5 4 2 2 4} 4 \mathbf{4 4 1 2 0 0}$ & $\mathbf{3 3 2 . 4 1 6 5}$ & $\mathbf{8 . 6 6 7 5}$ & $\mathbf{0 . 2 6 8 7}$ \\
$\mathbf{1 5 5 4 0 0} 4 \mathbf{1 1 0 0 0 0}$ & $\mathbf{3 3 6 . 1 3 6 4}$ & $\mathbf{9 . 8 1 5 8}$ & $\mathbf{0 . 4 1 7 9}$ \\
155959003000 & 9.4834 & 36.974 & 0.1462 \\
$\mathbf{1 6 0 3 1 2} 455359$ & $\mathbf{3 3 4 . 1 7 6}$ & $\mathbf{5 . 0 1 7 4}$ & $\mathbf{1 . 2 4 3 6}$ \\
$\mathbf{1 7 0 0 2 4} \mathbf{0 5 1 2 0 0}$ & $\mathbf{1 4 . 5 9 1 8}$ & $\mathbf{2 1 . 8 0 6}$ & $\mathbf{0 . 4 7 8 4}$ \\
$\mathbf{1 7 3 3 5 9 4 3 0 0 0 0}$ & $\mathbf{3 4 6 . 8 1 0 4}$ & $\mathbf{- 5 . 4 6 0 4}$ & $\mathbf{0 . 6 3 8 6}$ \\
181959470000 & 347.1854 & -14.5017 & 0.0945 \\
200246454536 & 353.8071 & -31.1154 & 0.0482 \\
201959470000 & 352.7417 & -34.2074 & 0.0419 \\
471200 & 352.6892 & -37.6217 & 0.0366
\end{tabular}




\begin{tabular}{lccc}
\multicolumn{4}{l}{ continued from previous page } \\
\hline \hline Field & $\begin{array}{c}\text { l } \\
\left(^{\circ}\right)\end{array}$ & $\begin{array}{c}\text { b } \\
\left(^{\circ}\right)\end{array}$ & Mean E(B-V) \\
\hline 211000020000 & 48.4331 & -31.4288 & 0.0551 \\
211959480000 & 351.1175 & -44.2975 & 0.0375 \\
212800020000 & 51.2491 & -35.2688 & 0.0588 \\
215200033000 & 53.7985 & -41.1081 & 0.0445 \\
215959480000 & 349.0457 & -50.8337 & 0.0257 \\
224135480000 & 344.574 & -57.2816 & 0.0093 \\
230000470000 & 343.2279 & -60.4697 & 0.0116 \\
232000020000 & 77.9707 & -56.6649 & 0.0422 \\
232000480000 & 337.3118 & -62.5768 & 0.0089 \\
233000050000 & 78.0953 & -60.5843 & 0.0432 \\
234000010000 & 86.6902 & -58.7474 & 0.0319 \\
234000470000 & 333.4433 & -65.6767 & 0.0137 \\
235000050000 & 86.7355 & -63.4716 & 0.035 \\
235600020000 & 92.7531 & -61.5758 & 0.0341 \\
235600480000 & 326.6305 & -66.4947 & 0.0113 \\
\hline \hline
\end{tabular}

We have reddened simulations using the Schlegel extinction maps, because the reddening at these direction is low in average, assuming a relation between the reddening and total extinction in each band given by table 2 of Catelan et al. (2011) (Table 9.3) which uses the Cardelli et al. (1989) extinction law. We have checked where the extinction is higher by comparing color distributions.

Table 9.3:

Total extinction in each filter

\begin{tabular}{lc}
\hline \hline Filter & $\mathrm{A}_{x} / \mathrm{E}(\mathrm{B}-\mathrm{V})$ \\
\hline $\mathrm{J}$ & 0.866 \\
$\mathrm{H}$ & 0.567 \\
$\mathrm{~K}$ & 0.364 \\
\hline \hline
\end{tabular}

Figure 9.1, 9.2 and 9.3 show the reddening (left panel) and color (right panel) distributions for the six fields with $\mathrm{E}(\mathrm{B}-\mathrm{V})>0.25 \mathrm{mag}$. In the figures right panel black lines are observations and red lines simulations. The color distributions and the modes are given in the right panel in the observed and simulated catalogs.

From the color histograms and corresponding modes we consider the field 170024051200 $\left(l=14.6^{\circ}, b=21.8^{\circ}\right)$ as suitable to be included in our analysis because the color distribution is well reproduced and the modes of the observed and simulated distributions are very similar. We do not consider other high extinction fields for further analysis. 

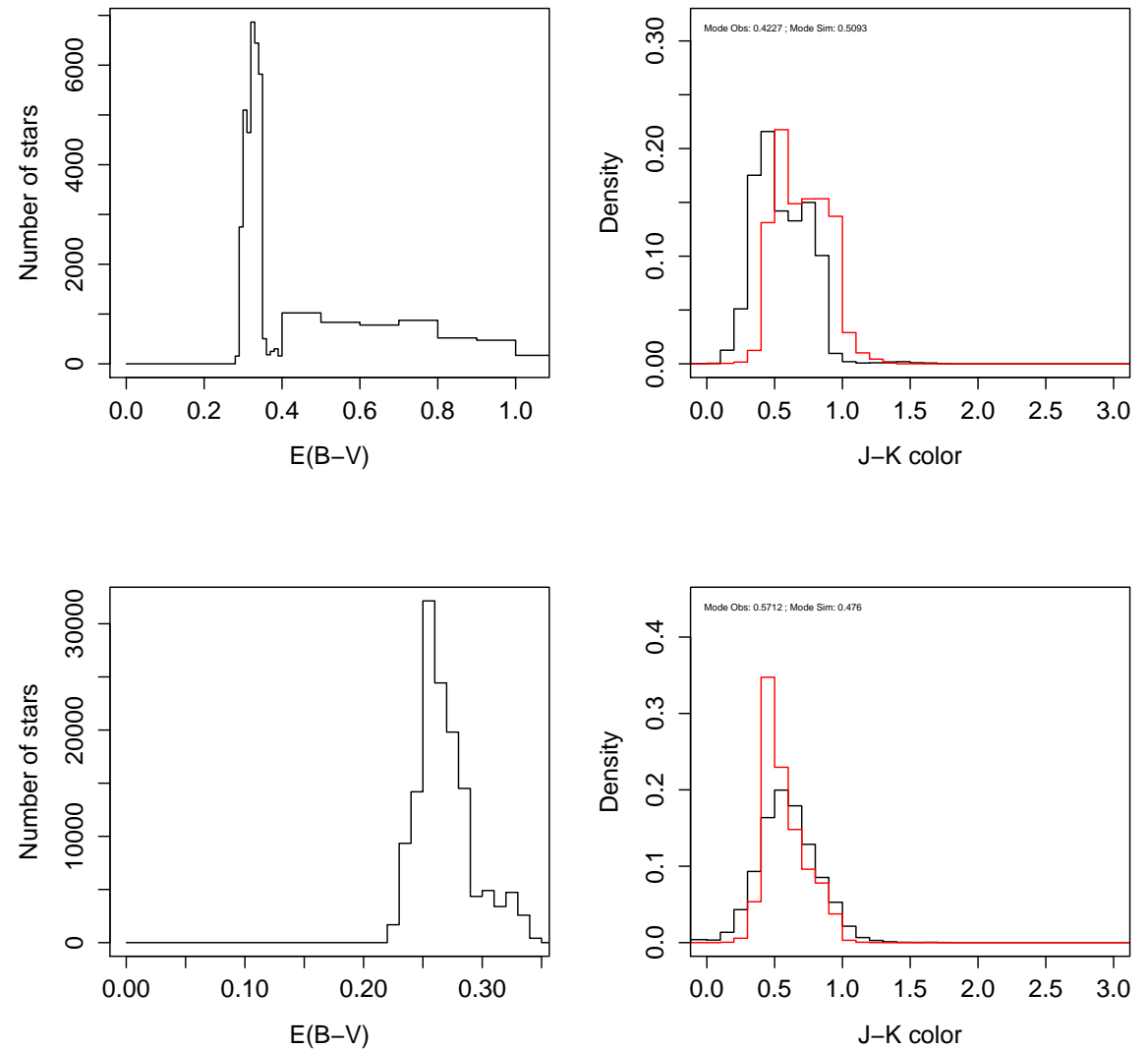

Figure 9.1: Reddening from SFD maps and color distributions from GES data (black lines) and simulations (red lines) for field 074500423000 (top panel) and field 154224441200 (bottom panel). 

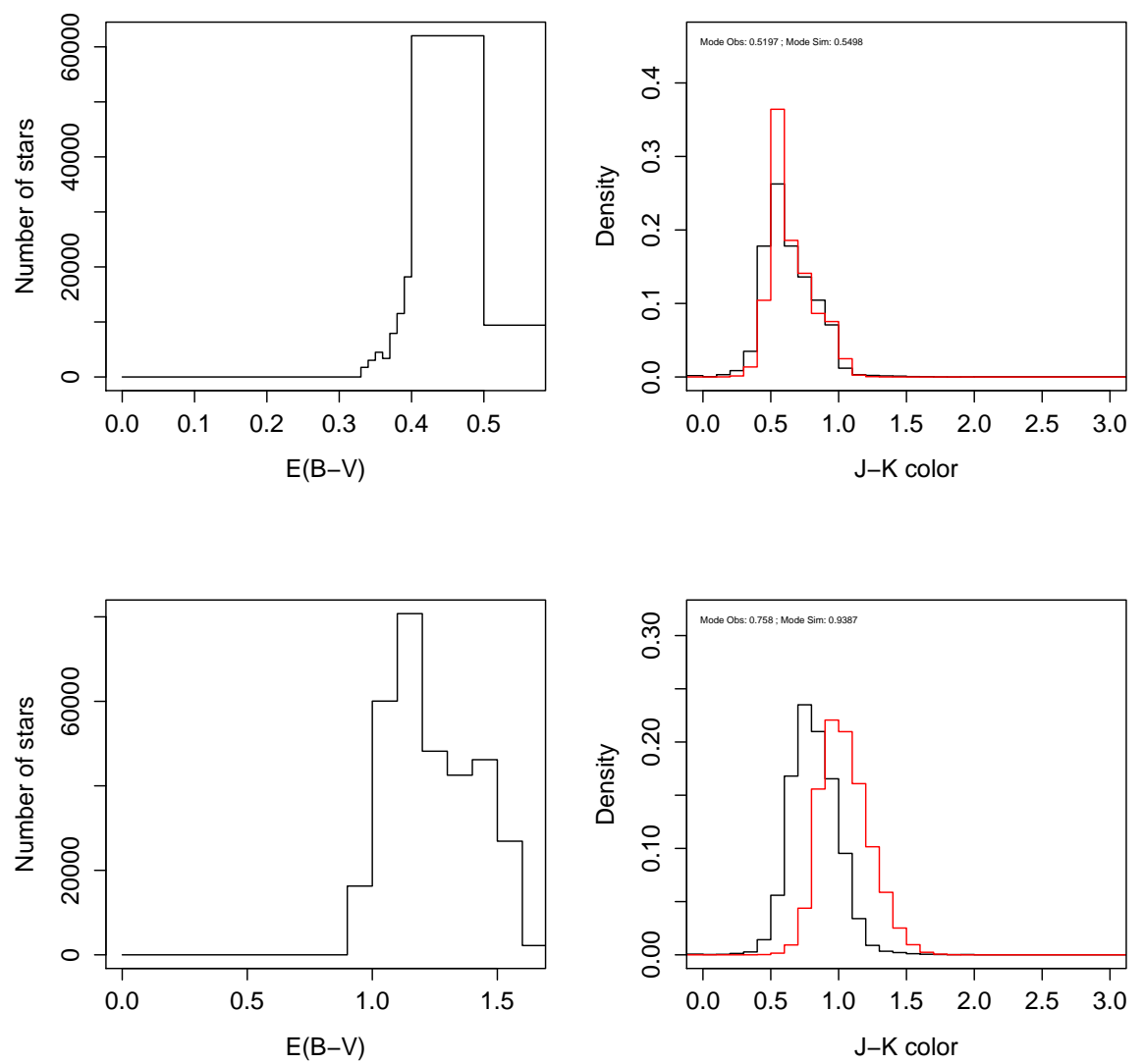

Figure 9.2: Reddening from SFD maps and color distributions from GES data (black lines) and simulations (red lines) for field 155400410000 (top panel) and field 160312455359 (bottom panel). 

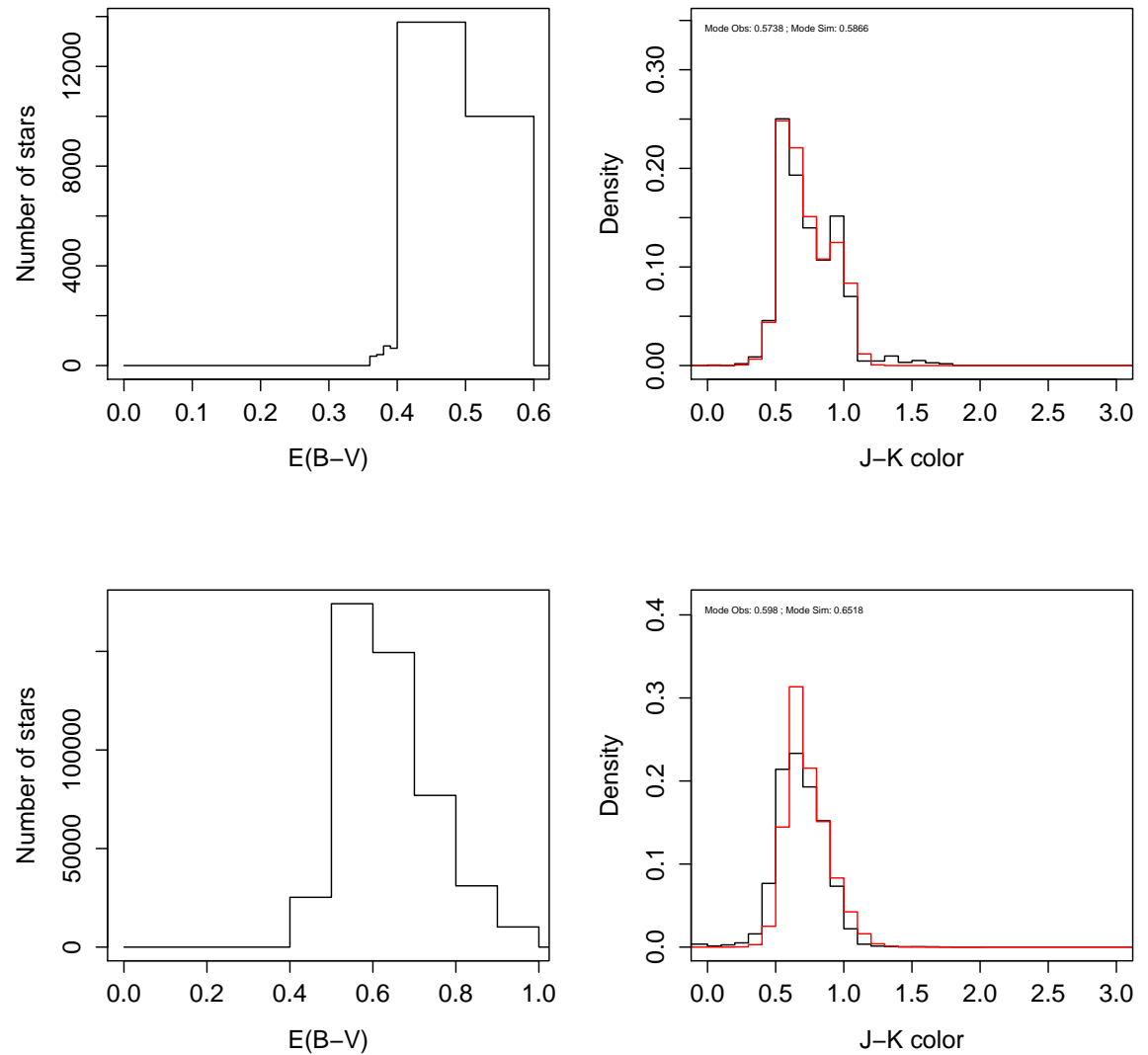

Figure 9.3: Reddening from SFD maps and color distributions from GES data (black lines) and simulations (red lines) for field 170024051200 (top panel) and field 173359430000 (bottom panel). 


\subsection{Spectroscopic errors}

The internal errors in metallicity, effective temperature and gravity are $0.08 \mathrm{dex}, 70 \mathrm{~K}$ and 0.10 dex respectively (Recio-Blanco et al. 2014). If we consider the external errors the uncertainties should be around $100 \mathrm{~K}$ in Teff, 0.15 dex in logg and 0.10 dex for $[\mathrm{M} / \mathrm{H}]$ (GES internal private communication). We have simulated the spectrocopic errors by assuming the external errors drawn from a Gaussian distribution.

\subsection{The selection sample}

We have selected FGK-type stars observed by GIRAFFE from the Gaia-ESO Survey. The selection function is based on the VISTA photometry. The target selection, for the disc fields, explained in Gilmore et al. (2012) is based on the Disc/Halo transition from the Sloan Digital Sky Survey photometry. There are two main boxes, a blue one and a red one

- Blue box: $0.0<(J-K)_{0}<0.45$ and $14.0<J_{0}<17.5$ mag

- Red box: $0.4<(J-K)_{0}<0.70$ and $12.5<J_{0}<15.0 \mathrm{mag}$

- Extended red box: $0.4<(J-K)_{0}<0.85$ and $12.5<J_{0}<17.0$ mag (when the number of stars was not enough to fill all FLAMES fibers)

These boxes are defined according to the Schlegel extinction maps (Schlegel et al. 1998) by shifting the box by an amount corresponding to the average total extinction in the field. The observed GES catalog that we use has been given by the GES consortium (GES internal release DR2).

After applying the extinction to the simulated stars, we selected stars in the simulated catalog by binning in the color (J-K) magnitude $(\mathrm{J})$ space, with bins of 0.05 and 0.1 mags respectively, and selecting the same number of stars present in each bin of the color magnitude bins of the observations. The total number of stars in both catalogs is 7470 . In table 9.4 we show the number of thin, young thick, old thick disc and total number of stars, selected from the populations in the model, for each field in the simulated catalogs.

Table 9.4:

Number of stars, in the model, for the thin, young thick, old thick disc for each field after applying the selection sample. The total number of stars in each field is given in the last column 


\begin{tabular}{|c|c|c|c|c|c|c|}
\hline Field & $1\left(^{\circ}\right)$ & $\mathrm{b}\left({ }^{\circ}\right)$ & thin disc & young thick disc & old thick disc & total \\
\hline 000400010000 & 97 & -61 & 15 & 15 & 15 & 49 \\
\hline 000400470000 & 325 & -68 & 5 & 18 & 11 & 39 \\
\hline 002000010000 & 105 & -63 & 10 & 22 & 14 & 53 \\
\hline 002000470000 & 319 & -69 & 6 & 8 & 10 & 30 \\
\hline 002959033000 & 110 & -66 & 13 & 12 & 16 & 43 \\
\hline 002959500600 & 311 & -67 & 16 & 20 & 20 & 64 \\
\hline 003240440056 & 314 & -73 & 8 & 10 & 11 & 30 \\
\hline 004000010000 & 117 & -64 & 9 & 8 & 7 & 26 \\
\hline 004000470000 & 309 & -70 & 1 & 4 & 6 & 11 \\
\hline 005000033000 & 122 & -66 & 9 & 13 & 10 & 34 \\
\hline 005000500600 & 304 & -67 & 10 & 19 & 16 & 50 \\
\hline 005959010000 & 128 & -64 & 7 & 10 & 8 & 26 \\
\hline 005959470000 & 298 & -70 & 12 & 12 & 19 & 47 \\
\hline 011959010000 & 139 & -63 & 6 & 11 & 10 & 28 \\
\hline 011959470000 & 289 & -69 & 13 & 15 & 14 & 47 \\
\hline 014000010000 & 149 & -61 & 10 & 15 & 15 & 45 \\
\hline 014000470000 & 280 & -68 & 10 & 25 & 17 & 60 \\
\hline 015959010000 & 158 & -59 & 11 & 19 & 11 & 44 \\
\hline 015959470000 & 273 & -66 & 13 & 23 & 15 & 55 \\
\hline 022000470000 & 268 & -64 & 5 & 17 & 9 & 35 \\
\hline 023000033000 & 172 & -57 & 14 & 15 & 10 & 40 \\
\hline 023959010000 & 172 & -53 & 6 & 23 & 6 & 36 \\
\hline 023959470000 & 263 & -61 & 4 & 9 & 9 & 23 \\
\hline 025556002846 & 176 & -50 & 10 & 13 & 10 & 34 \\
\hline 025559003000 & 176 & -50 & 17 & 14 & 5 & 36 \\
\hline 030000470000 & 260 & -58 & 19 & 18 & 11 & 53 \\
\hline 030959500600 & 263 & -55 & 15 & 17 & 26 & 63 \\
\hline 031800003000 & 182 & -46 & 9 & 19 & 9 & 39 \\
\hline 032000470000 & 257 & -55 & 12 & 17 & 23 & 60 \\
\hline 033000500600 & 261 & -52 & 12 & 18 & 15 & 47 \\
\hline 033800273000 & 223 & -53 & 7 & 17 & 18 & 45 \\
\hline 033959000000 & 186 & -41 & 17 & 17 & 10 & 45 \\
\hline 033959470000 & 255 & -52 & 17 & 18 & 16 & 53 \\
\hline 035959470000 & 254 & -48 & 11 & 14 & 21 & 48 \\
\hline 041000500600 & 258 & -46 & 19 & 25 & 9 & 57 \\
\hline 041959001959 & 194 & -33 & 27 & 22 & 27 & 78 \\
\hline 043000500600 & 257 & -43 & 15 & 20 & 16 & 61 \\
\hline 044000500600 & 257 & -41 & 9 & 23 & 17 & 51 \\
\hline 050000520000 & 259 & -38 & 18 & 36 & 25 & 88 \\
\hline 051959540000 & 262 & -35 & 21 & 32 & 20 & 77 \\
\hline
\end{tabular}




\begin{tabular}{|c|c|c|c|c|c|c|}
\hline Field & $1\left(^{\circ}\right)$ & $\overline{\mathrm{b}\left({ }^{\circ}\right)}$ & thin disc & young thick disc & old thick disc & $\overline{\text { total }}$ \\
\hline 070359423000 & 253 & -16 & 36 & 25 & 14 & 76 \\
\hline 072048003000 & 217 & 6 & 73 & 18 & 0 & 91 \\
\hline 075600090000 & 228 & 10 & 55 & 19 & 9 & 83 \\
\hline 075959003000 & 221 & 15 & 52 & 36 & 16 & 106 \\
\hline 082312052959 & 229 & 18 & 29 & 30 & 18 & 78 \\
\hline 083959003000 & 227 & 24 & 20 & 41 & 24 & 85 \\
\hline 092758003043 & 234 & 34 & 22 & 28 & 18 & 69 \\
\hline 092800003000 & 234 & 34 & 22 & 24 & 25 & 74 \\
\hline 094753102657 & 247 & 32 & 4 & 18 & 9 & 34 \\
\hline 095958410150 & 271 & 11 & 55 & 20 & 4 & 79 \\
\hline 100000410000 & 271 & 11 & 36 & 31 & 17 & 86 \\
\hline 100759080000 & 249 & 37 & 20 & 23 & 14 & 58 \\
\hline 100913412801 & 273 & 12 & 38 & 31 & 10 & 80 \\
\hline 101428405235 & 274 & 13 & 36 & 46 & 10 & 94 \\
\hline 102800410000 & 276 & 14 & 36 & 29 & 12 & 77 \\
\hline 103959410000 & 278 & 15 & 36 & 21 & 5 & 63 \\
\hline 105731124726 & 264 & 42 & 9 & 21 & 16 & 48 \\
\hline 105808154324 & 267 & 39 & 3 & 25 & 20 & 51 \\
\hline 105959410000 & 281 & 17 & 39 & 35 & 25 & 101 \\
\hline 110009410232 & 281 & 17 & 39 & 32 & 10 & 82 \\
\hline 110053132816 & 266 & 41 & 3 & 11 & 20 & 41 \\
\hline 112200100000 & 270 & 47 & 10 & 26 & 26 & 66 \\
\hline 112800410000 & 286 & 19 & 21 & 34 & 14 & 71 \\
\hline 113159435959 & 288 & 17 & 34 & 40 & 10 & 86 \\
\hline 120000090000 & 282 & 52 & 8 & 24 & 17 & 52 \\
\hline 120000410000 & 293 & 21 & 20 & 41 & 29 & 91 \\
\hline 121159410000 & 295 & 21 & 22 & 30 & 17 & 69 \\
\hline 122759410000 & 298 & 22 & 15 & 33 & 12 & 62 \\
\hline 124224130559 & 300 & 50 & 7 & 22 & 24 & 55 \\
\hline 124359060000 & 300 & 57 & 11 & 18 & 23 & 57 \\
\hline 124359090000 & 300 & 54 & 11 & 27 & 19 & 61 \\
\hline 124359410000 & 301 & 22 & 25 & 28 & 6 & 59 \\
\hline 125609451238 & 304 & 18 & 31 & 34 & 10 & 75 \\
\hline 130047410000 & 305 & 22 & 18 & 50 & 21 & 93 \\
\hline 131359410000 & 308 & 22 & 17 & 30 & 9 & 56 \\
\hline 131359460007 & 307 & 17 & 15 & 35 & 8 & 61 \\
\hline 132000050000 & 316 & 57 & 13 & 18 & 24 & 62 \\
\hline 132000090000 & 315 & 53 & 9 & 17 & 17 & 52 \\
\hline 132000130000 & 313 & 49 & 13 & 12 & 9 & 37 \\
\hline 132800410000 & 310 & 21 & 21 & 35 & 11 & 69 \\
\hline 133026434759 & 310 & 19 & 15 & 37 & 8 & 61 \\
\hline
\end{tabular}


continued from previous page

\begin{tabular}{|c|c|c|c|c|c|c|}
\hline Field & $\overline{l 1\left(^{\circ}\right)}$ & $\overline{\mathrm{b}\left({ }^{\circ}\right)}$ & thin disc & young thick disc & old thick disc & total \\
\hline 134400410000 & 313 & 21 & 18 & 48 & 17 & 85 \\
\hline 140000100000 & 329 & 49 & 10 & 19 & 23 & 61 \\
\hline 140000410000 & 317 & 20 & 21 & 49 & 19 & 92 \\
\hline 140000450000 & 315 & 16 & 22 & 46 & 11 & 79 \\
\hline 142000050000 & 340 & 51 & 9 & 18 & 13 & 45 \\
\hline 142000410000 & 320 & 19 & 21 & 40 & 12 & 74 \\
\hline 142145440827 & 320 & 16 & 17 & 37 & 8 & 65 \\
\hline 142800090000 & 339 & 47 & 10 & 29 & 31 & 80 \\
\hline 143959410000 & 324 & 17 & 23 & 40 & 21 & 86 \\
\hline 144113400831 & 325 & 18 & 23 & 34 & 23 & 83 \\
\hline 145800410000 & 327 & 16 & 18 & 25 & 12 & 58 \\
\hline 150159100000 & 348 & 41 & 9 & 37 & 34 & 91 \\
\hline 150512400000 & 329 & 16 & 49 & 44 & 9 & 104 \\
\hline 151111404800 & 330 & 15 & 41 & 40 & 17 & 101 \\
\hline 151712413000 & 330 & 14 & 44 & 43 & 15 & 106 \\
\hline 152311421200 & 331 & 12 & 43 & 37 & 14 & 95 \\
\hline 152936425359 & 331 & 11 & 31 & 41 & 13 & 89 \\
\hline 154224441200 & 332 & 9 & 38 & 53 & 14 & 107 \\
\hline 155959003000 & 9 & 37 & 7 & 23 & 22 & 64 \\
\hline 170024051200 & 15 & 22 & 12 & 38 & 7 & 60 \\
\hline 181959470000 & 347 & -14 & 26 & 35 & 14 & 79 \\
\hline 200246454536 & 354 & -31 & 8 & 44 & 36 & 101 \\
\hline 201959470000 & 353 & -34 & 7 & 13 & 6 & 26 \\
\hline 204000471200 & 353 & -38 & 4 & 38 & 26 & 73 \\
\hline 211000020000 & 48 & -31 & 18 & 41 & 24 & 89 \\
\hline 211959480000 & 351 & -44 & 9 & 36 & 33 & 86 \\
\hline 212800020000 & 51 & -35 & 13 & 29 & 18 & 63 \\
\hline 215200033000 & 54 & -41 & 16 & 25 & 24 & 72 \\
\hline 215959480000 & 349 & -51 & 8 & 27 & 36 & 80 \\
\hline 224135480000 & 344 & -57 & 15 & 28 & 17 & 62 \\
\hline 230000470000 & 343 & -60 & 9 & 21 & 16 & 49 \\
\hline 232000020000 & 78 & -57 & 18 & 22 & 16 & 63 \\
\hline 232000480000 & 337 & -63 & 6 & 8 & 5 & 22 \\
\hline 233000050000 & 78 & -61 & 13 & 18 & 20 & 58 \\
\hline 234000010000 & 87 & -59 & 9 & 16 & 20 & 48 \\
\hline 234000470000 & 333 & -66 & 9 & 12 & 16 & 44 \\
\hline 235000050000 & 87 & -63 & 9 & 22 & 19 & 52 \\
\hline 235600020000 & 93 & -62 & 12 & 20 & 7 & 42 \\
\hline 235600480000 & 327 & -66 & 8 & 11 & 9 & 35 \\
\hline
\end{tabular}




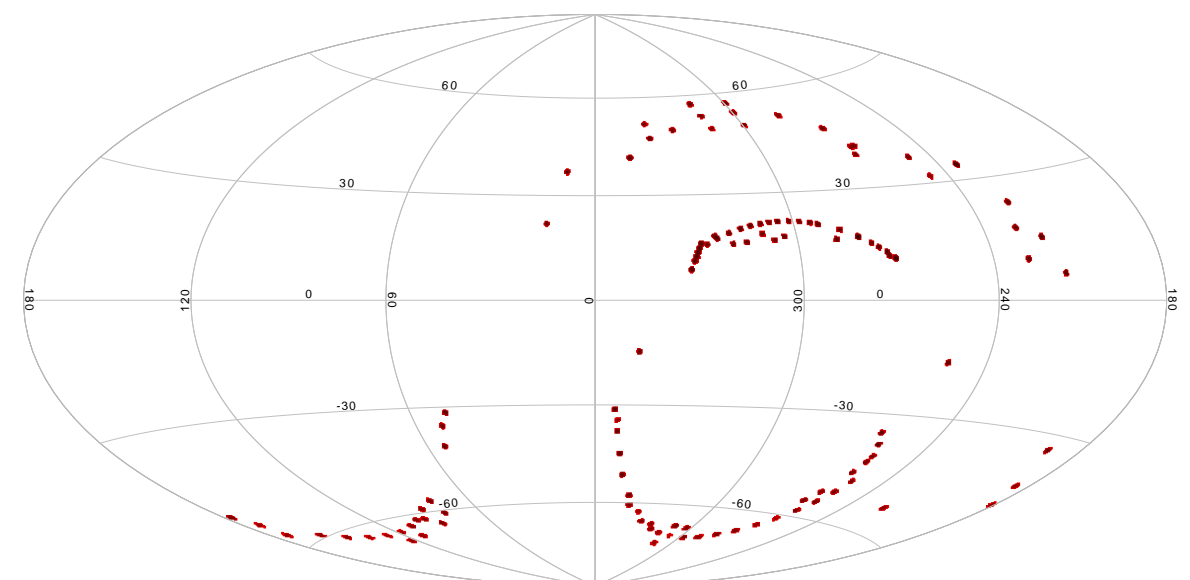

Figure 9.4: Aitoff projection for the iDR2 fields selected for this analysis.

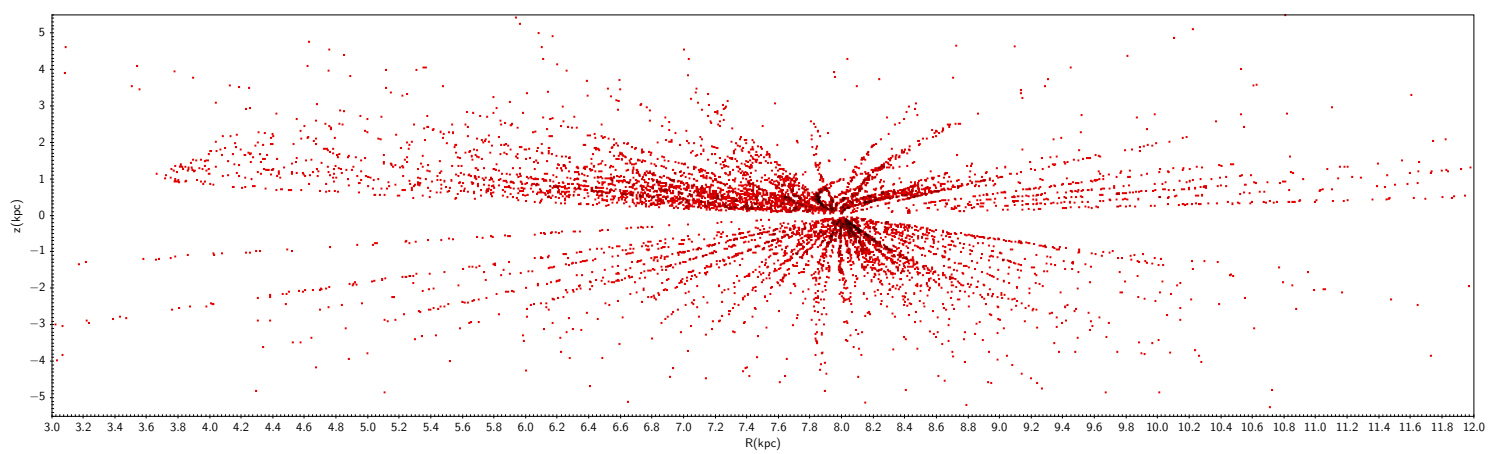

Figure 9.5: Spatial distribution for the F/G/K stars sample from GES as given by the simulations.

Figure 9.4 shows the Aitoff projection for the iDR2 fields selected for this analysis and figure 9.5 shows the spatial coverage in Galactocentric cylindrical coordinates for the $\mathrm{F} / \mathrm{G} / \mathrm{K}$ stars in the GES sample as given by the model. The sample covers a galactocentric distance in the range $4.0<\mathrm{Rgal}<10.5 \mathrm{kpc}$ and a distance above the plane in the range $-3.0<\mathrm{Z}<3.0$ kpc. The SEGUE sample goes further in the outer regions which allows a better analysis of the radial metallicity distribution in the external galaxy while GES sample covers better the inner regions. The $\mathrm{z}$ distribution in GES is larger than in SEGUE which allows a possible study of the vertical metallicity gradients.

\subsection{Distances}

We have calculated distances in a similar way as described in section 8.1.4. While in SEGUE we used the absolute magnitudes in V band for the GES sample we used absolute magnitudes in $\mathrm{J}$ band. From the SEGUE selection we selected MSTO stars while from GES disc selection 
Table 9.5:

Set of parameters used to compute absolute magnitude from the relation with effective temperature for dwarfs $(\operatorname{logg}>4.5 \mathrm{dex})$ and giants $(\operatorname{logg}<4.0 \mathrm{dex})$ and with $\log \mathrm{g}$ for subgiants (4.0 dex $\leq \operatorname{logg} \leq 4.5 \mathrm{dex}$ ). The selection in metallicity and $\log \mathrm{g}$ is given in the first two columns respectively.

\begin{tabular}{lcccc}
\hline \hline $\begin{array}{l}{[\mathrm{Fe} / \mathrm{H}] \text { range }} \\
(\mathrm{dex})\end{array}$ & $\begin{array}{c}\text { log g range } \\
(\mathrm{dex})\end{array}$ & Intercept & First coef & Second coef \\
\hline$[\mathrm{Fe} / \mathrm{H}]<-0.5$ & $\log >4.5$ & 17.384 & -0.004 & $2.29 \times 10^{-07}$ \\
$-0.5 \leq[\mathrm{Fe} / \mathrm{H}] \leq 0.0$ & $\log >4.5$ & 21.307 & -0.006 & $4.41 \times 10^{-07}$ \\
{$[\mathrm{Fe} / \mathrm{H}]>0.0$} & $\log >4.0$ & 20.904 & -0.005 & $3.72 \times 10^{-07}$ \\
{$[\mathrm{Fe} / \mathrm{H}]<-0.5$} & $\log <4.0$ & -39.826 & 0.013 & $-9.94 \times 10^{-07}$ \\
$-0.5 \leq[\mathrm{Fe} / \mathrm{H}] \leq 0.0$ & $\log <4.0$ & -51.826 & 0.018 & $-1.537 \times 10^{-06}$ \\
{$[\mathrm{Fe} / \mathrm{H}]>0.0$} & $\log \leq 4.0$ & -48.973 & 0.018 & $-1.537 \times 10^{-06}$ \\
{$[\mathrm{Fe} / \mathrm{H}] \leq-0.5$} & $4.0 \leq \operatorname{logg} \leq 4.5$ & 14.481 & -7.833 & 1.224 \\
{$[\mathrm{Fe} / \mathrm{H}]>-0.5$} & $4.0 \leq \operatorname{logg} \leq 4.5$ & 3.101 & -3.157 & 0.752 \\
\hline \hline
\end{tabular}

sample we selected in color range which contains a mixture of dwarfs, giants and subgiants. Therefore to compute the distance modulus for this sample, one needs a relation between absolute magnitude and effective temperature for each type of stars. Besides selecting stars in different metallicity ranges we have also to use the log g information to select giants, subgiants and dwarfs. For the giants and dwarfs we fitted the absolute magnitude as a function of temperature for each metallicity selection while for the subgiants we fitted the absolute magnitude as a function of the $\log \mathrm{g}$. We selected stars from which we computed absolute magnitudes in the ranges defined by metallicity and logg. For logg the selected intervals are smaller than 4.0 dex, between 4.0 dex and 4.5 dex, and larger than 4.5 dex. We make 3 groups of metallicities $([\mathrm{Fe} / \mathrm{H}]<-0.5,-0.5 \leq[\mathrm{Fe} / \mathrm{H}] \leq 0.0$ and $[\mathrm{Fe} / \mathrm{H}]>0.0)$, except for the subgiants $(4.0<\operatorname{logg}$ $<4.5)$ for which 2 groups are considered $([\mathrm{Fe} / \mathrm{H}] \leq-0.5$ and $[\mathrm{Fe} / \mathrm{H}]>-0.5)$.

We have fitted with a second order polynomial each of these ranges for each of the luminosity class. Figure 9.6 and 9.7 show the absolute magnitude as a function of effective temperature and 9.8 show the absolute magnitude as a function of the $\log \mathrm{g}$ for the stars in the selected ranges. Table 9.5 shows the results of the fitted relations.

The distance modulus is computed, in $\mathrm{J}$ band, as explained in section 8.1.4 correcting the observed apparent magnitudes with Schlegel extinction values in J band. Figure 9.9 shows the difference between distance estimate and the distance given by the model as a function of the distance given by the model. The figure shows a good agreement between the distances computed from our method and the distances given by the BGM. In comparison with the distances computed from SEGUE there is a clear improvement. We can inspect the accuracy of our method in relation to the BGM distances from the distribution of the difference between distance estimate and the distance given by the model shown in figure 9.10. The results show that the accuracy is good with more than $91 \%$ of the stars inside $1.0 \mathrm{kpc}$ accuracy but there is a 

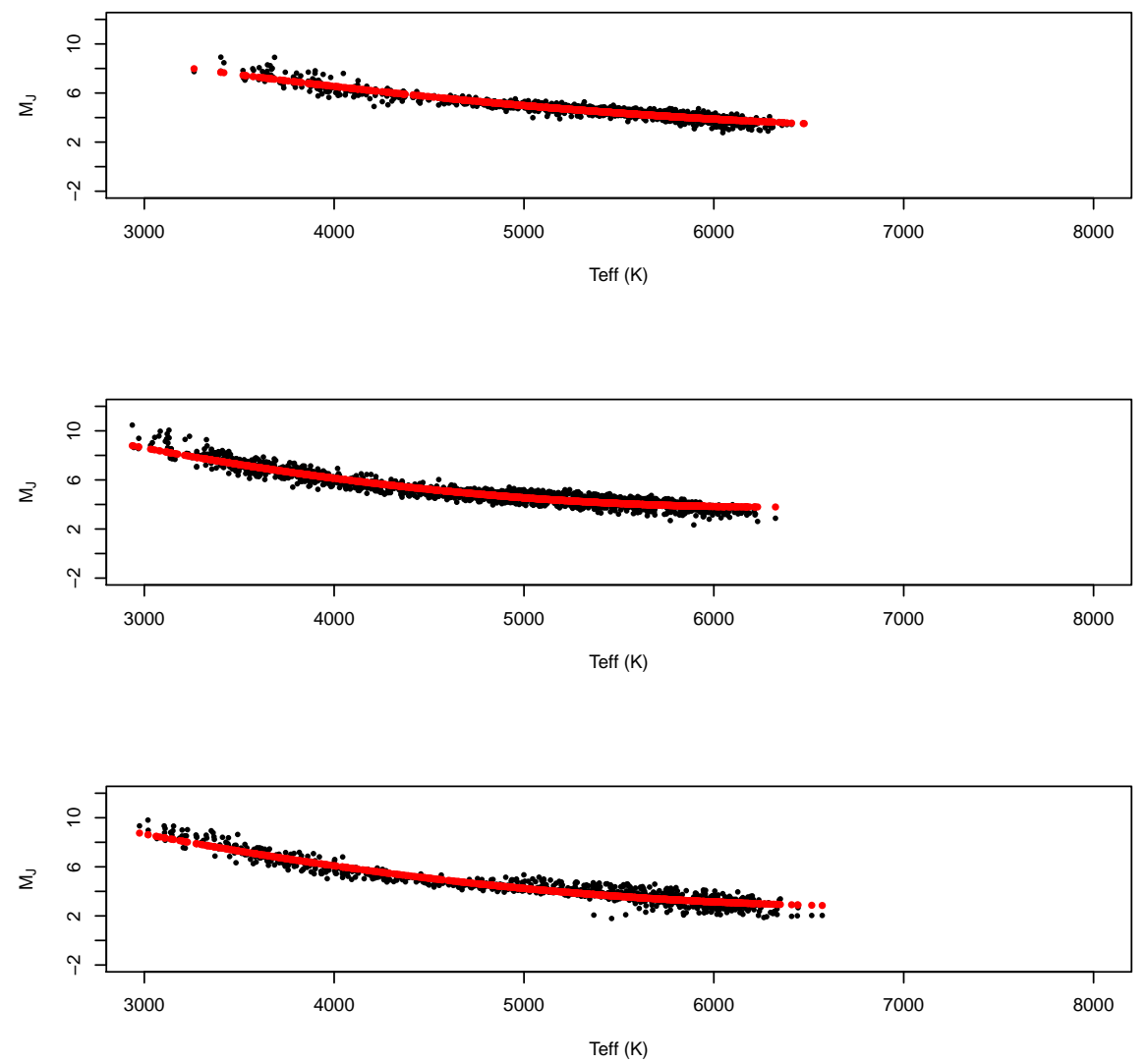

Figure 9.6: Absolute magnitude as a function of the effective temperature for dwarf stars in the three different metallicity ranges. From top to bottom the metallicity ranges are $[\mathrm{Fe} / \mathrm{H}]<-0.5,-0.5 \leq[\mathrm{Fe} / \mathrm{H}] \leq 0.0$ and $[\mathrm{Fe} / \mathrm{H}]>0.0$ respectively. 

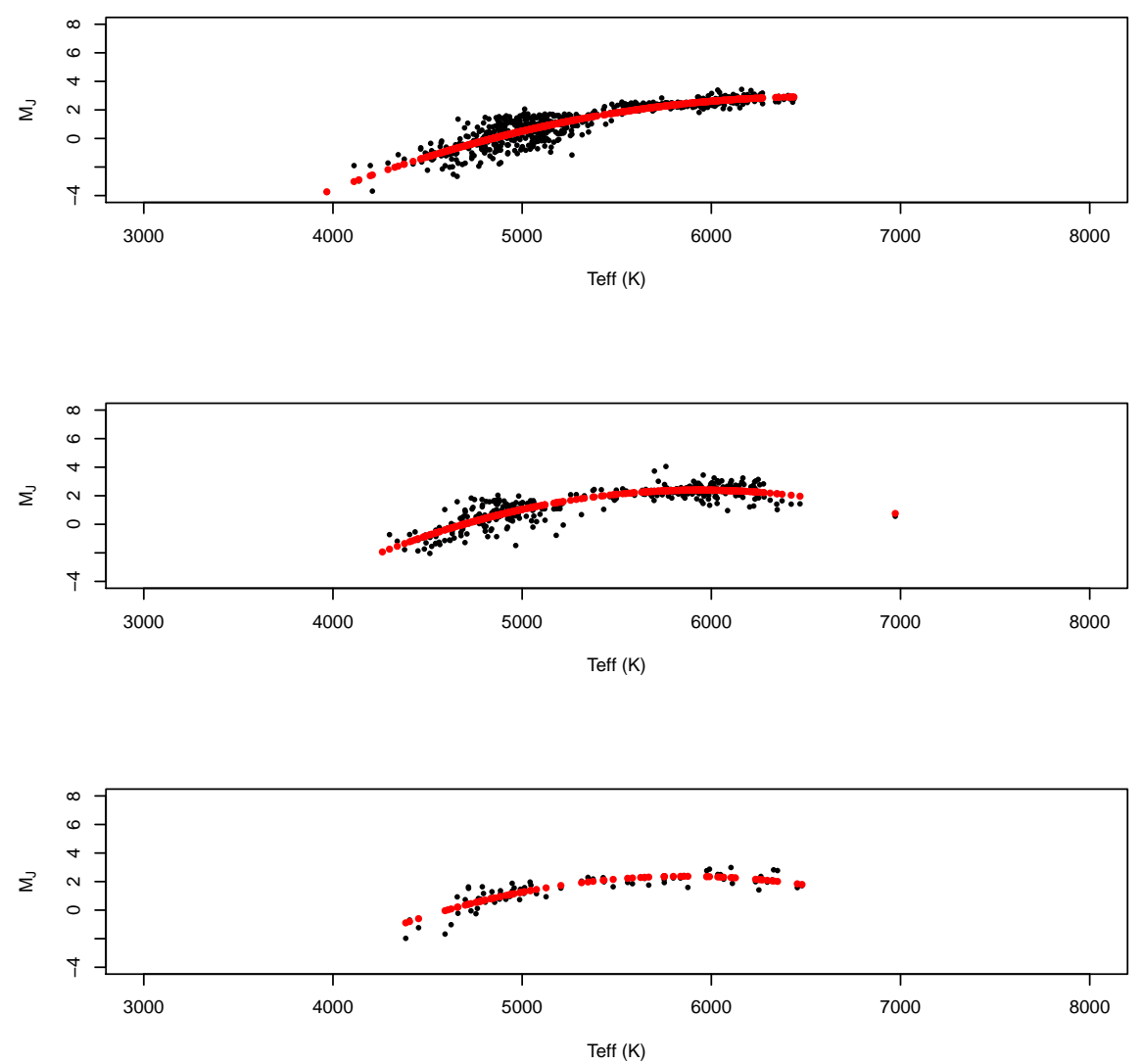

Figure 9.7: Absolute magnitude as a function of the effective temperature for giants stars in the three different metallicity ranges. From top to bottom the metallicity ranges are $[\mathrm{Fe} / \mathrm{H}]<-0.5,-0.5 \leq[\mathrm{Fe} / \mathrm{H}] \leq 0.0$ and $[\mathrm{Fe} / \mathrm{H}]>0.0$ respectively. 

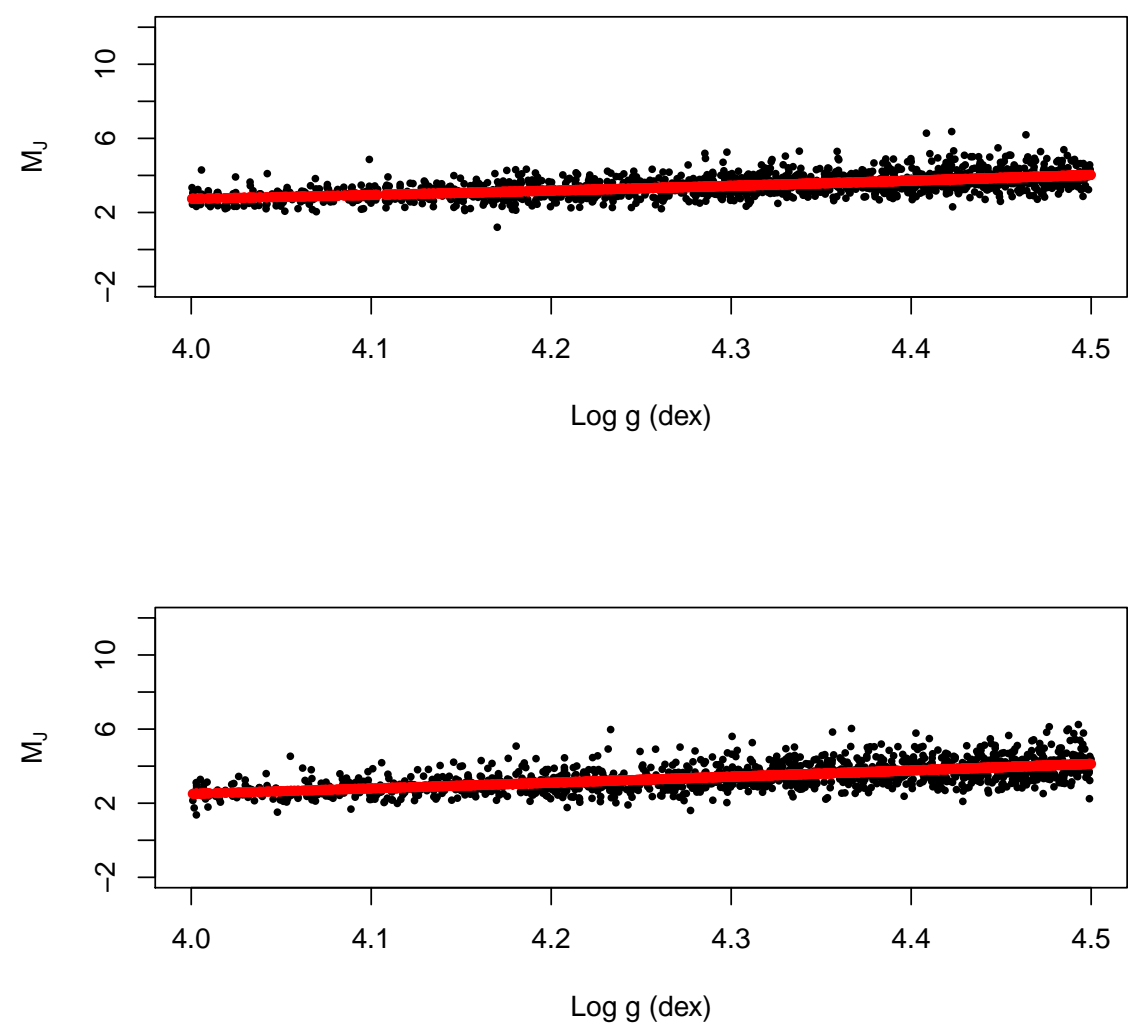

Figure 9.8: Absolute magnitude as a function of the log g for subgiants stars in the two different metallicity ranges. From top to bottom the metallicity ranges are $[\mathrm{Fe} / \mathrm{H}] \leq-0.5$ and $[\mathrm{Fe} / \mathrm{H}]>-0.5$ respectively. 


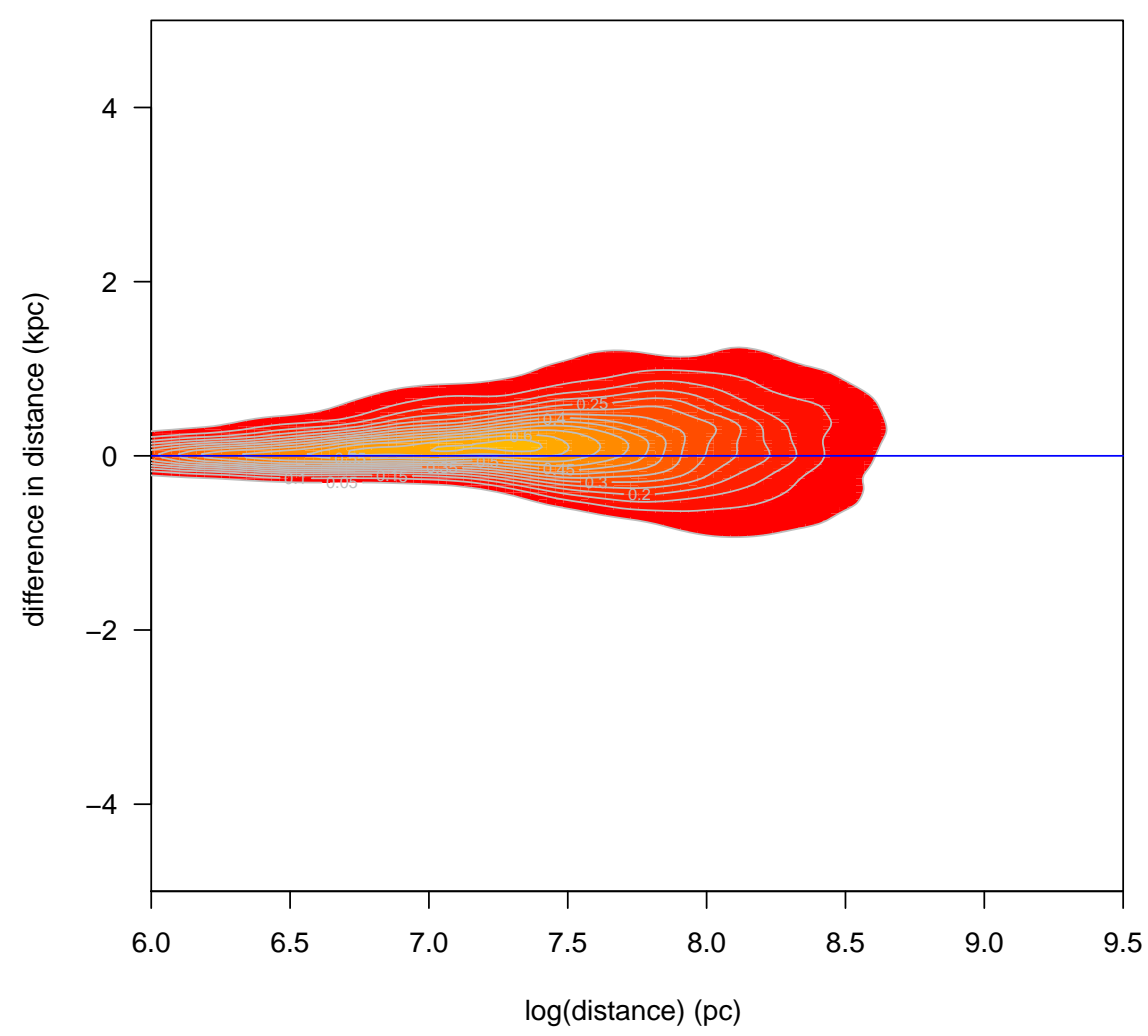

Figure 9.9: Difference between distance estimate and the distance given by the model as a function of the $\log$ (distance) (natural logarithm) given by the model. Density map and grey contours refer to our method.

bias towards larger distances computed from our method.

\subsection{The Metallicity Distribution}

\subsubsection{The observed metallicity distribution}

Figure 9.11 shows the metallicity distribution of the total GES sample. The total metallicity distribution which is the mixture essentially of the thin and thick disc has a mean equal to -0.294 dex with a dispersion of 0.468 dex with a low metallicity tail. 


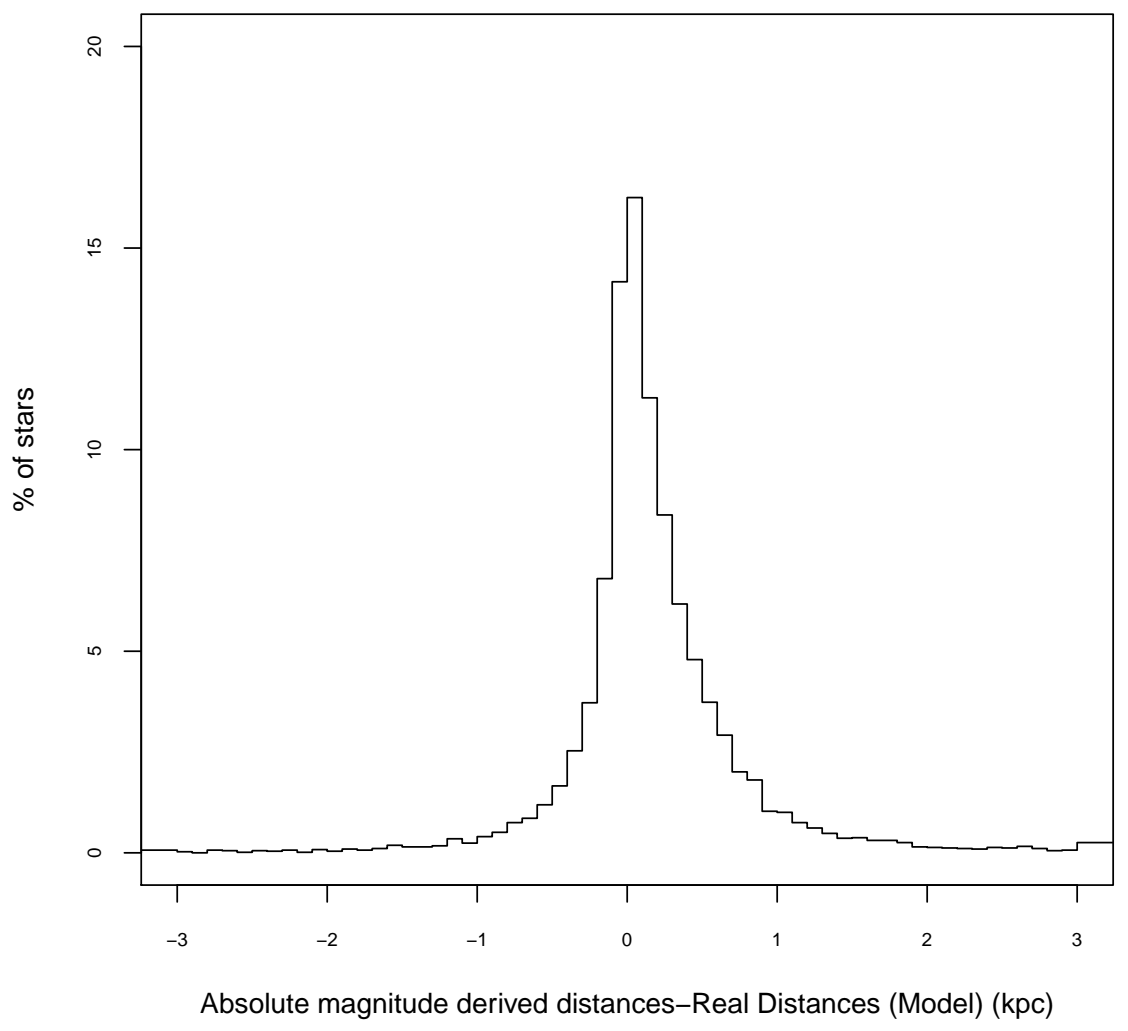

Figure 9.10: Distribution of the differences between distance estimate and the distance given by the model.

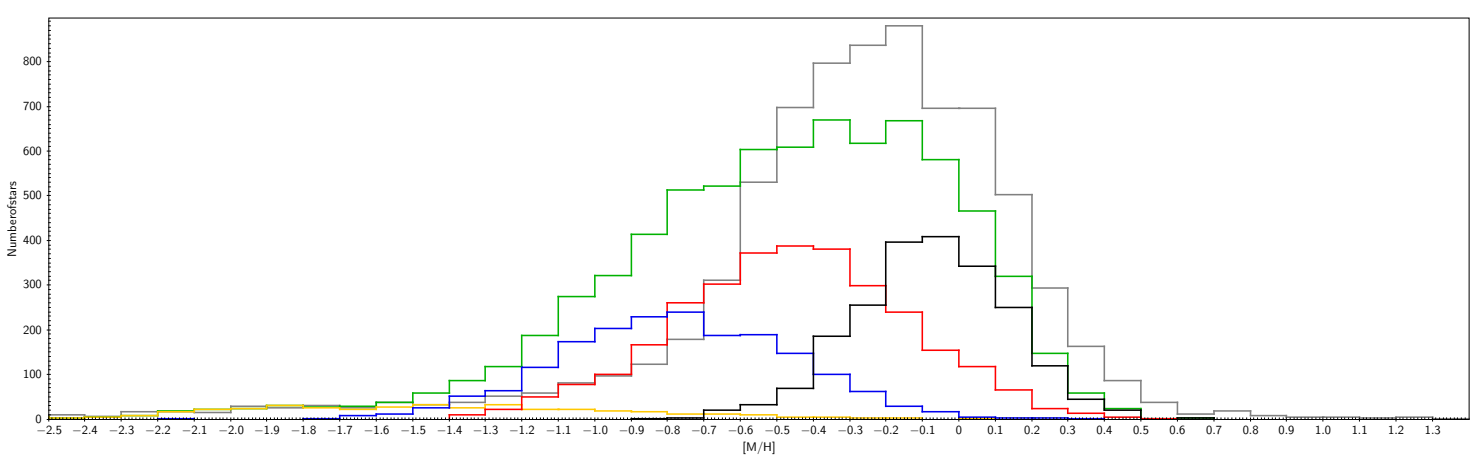

Figure 9.11: Metallicity distribution of the total GES sample from observations (grey lines) and as given by simulations (Green lines). The different components as given by the model are: black lines: Thin disc; red lines: Young thick disc; blue lines: Old thick disc; orange lines: Halo. 

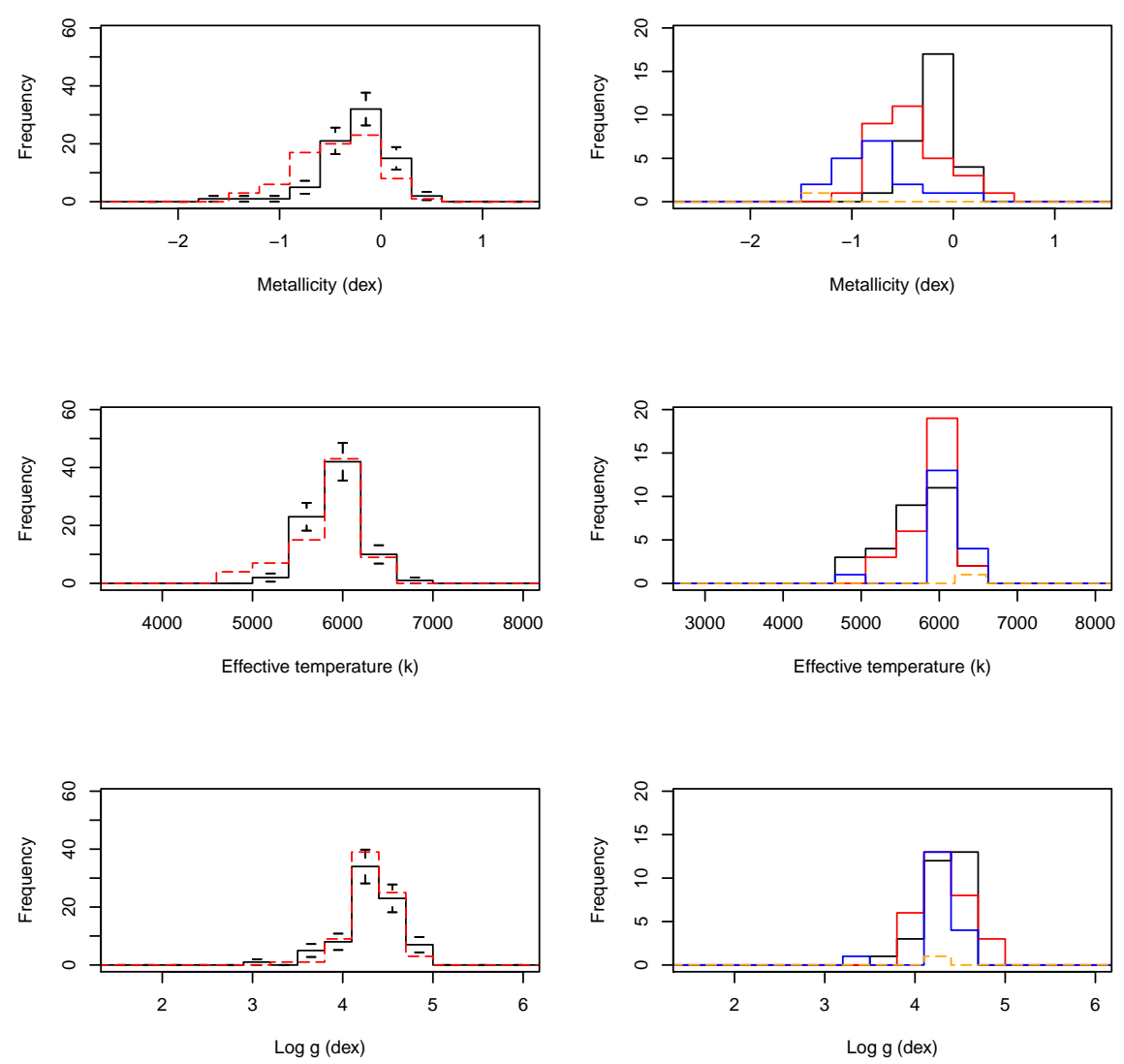

Figure 9.12: Comparison of the spectrocopic observations and simulations for a field at $1=229^{\circ}$ and $b=18^{\circ}$. Left panels: Black lines are observations and simulations are in red. The Poisson noise is represented by the small vertical bars. Right panels: Black lines are thin disc; red lines are young thick disc; blue lines are old thick disc; orange lines are halo.

\subsubsection{Preliminary comparison with the BGM}

The simulated spectral parameters distributions were compared with the observed spectrocopic distributions for the different fields. Figures 9.12 and 9.13 show the parameter distributions for field 082312052959 at the direction $l=229^{\circ}$ and $b=18^{\circ}$ and for field 000400010000 at the direction $1=97^{\circ}$ and $b=-61^{\circ}$ respectively. These two directions are located at nearly opposite places of the Milky Way. Field 082312052959 presents a good agreement for the effective temperature and $\log \mathrm{g}$ distributions. The simulated metallicity distribution is slightly lower than the observed one. Field 000400010000 presented in figure 9.13 presents a metallicity distribution similar to the former one. The effective temperature presents a wide distribution. Figure 9.14 shows the effective temperature as a function of the $\mathrm{J}-\mathrm{K}$ color. The figure shows an existing bias which may have come from the data, from the isochrones, that should be revised, or because the extinction was not corrected properly. The simulted $\log \mathrm{g}$ distribution is in agreement with the observational distribution at the $2 \sigma$ level. 

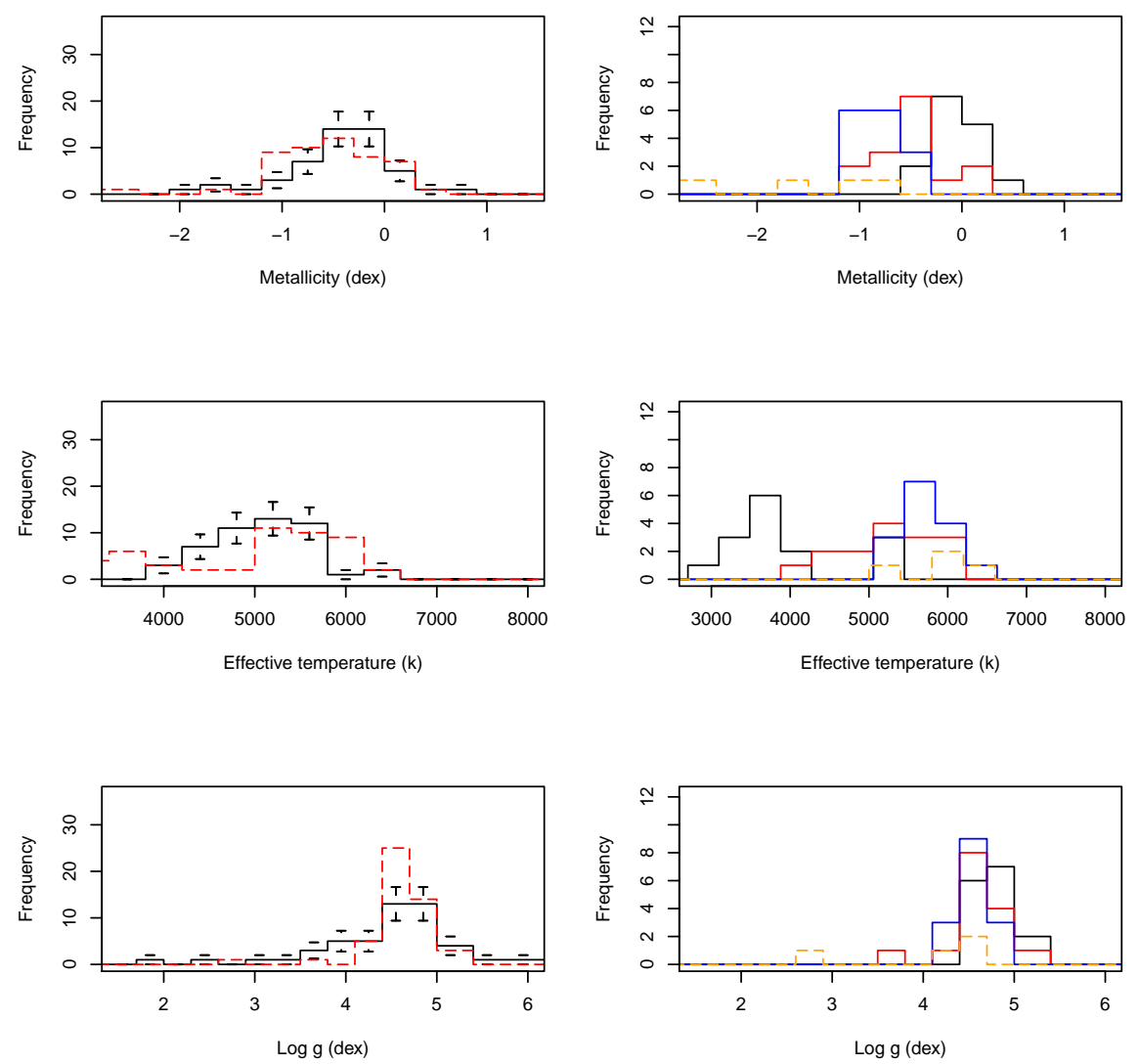

Figure 9.13: Comparison of the spectrocopic observations and simulations for a field at $1=97^{\circ}$ and $b=-61^{\circ}$. Left panels: Black lines are observations and simulations are in red. The Poisson noise is represented by the small vertical bars. Right panels: Black lines are thin disc; red lines are young thick disc; blue lines are old thick disc; orange lines are halo. 


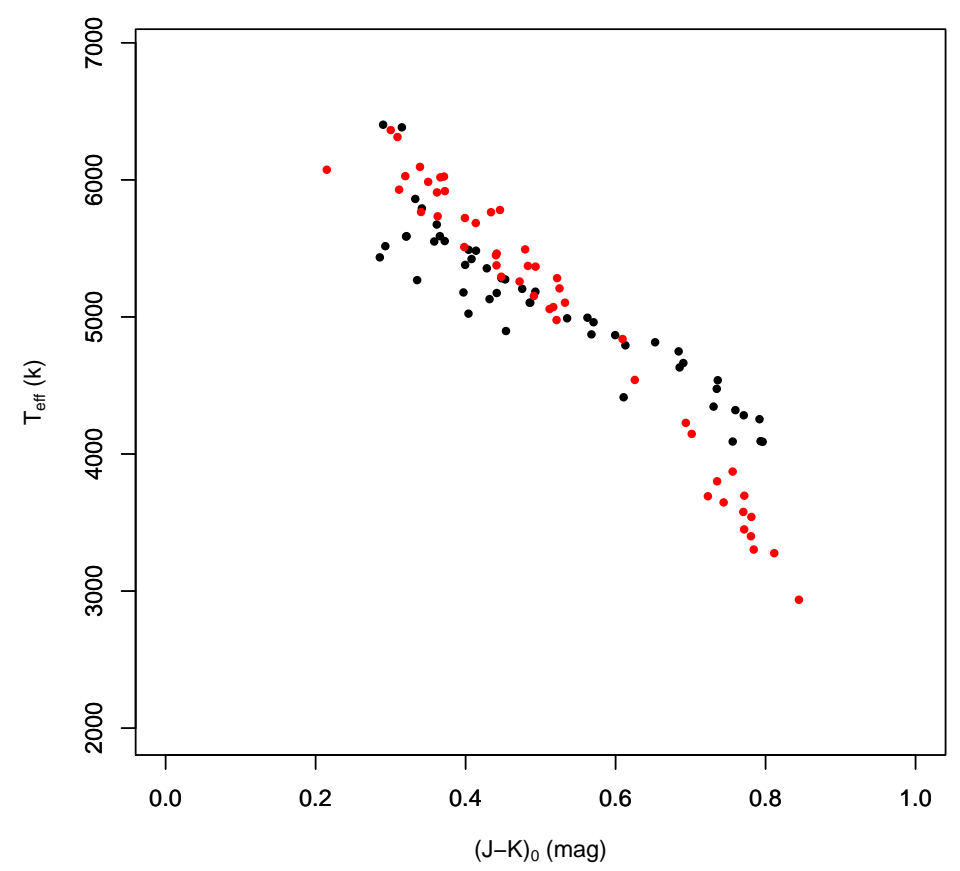

Figure 9.14: Effective temperature as a function of the $\mathrm{J}-\mathrm{K}$ color for a field at $\mathrm{l}=97^{\circ}$ and $\mathrm{b}=-61^{\circ}$. Black points represent observations and red points simulations. 
To inspect the metallicity distributions we computed the means, modes and dispersion in both catalogs for all fields. The results are presented in table 9.6. This table shows that the means and modes for the simulated metallicity distributions are often lower than the ones from the observed metallicity distribution.

Table 9.6:

GES metallicity distribution

\begin{tabular}{|c|c|c|c|c|c|c|c|c|}
\hline Field & $\begin{array}{l}\text { mean obs } \\
\quad(\text { dex })\end{array}$ & $\begin{array}{l}\text { mean sim } \\
\quad(\mathrm{dex})\end{array}$ & $\begin{array}{l}\text { mode obs } \\
\quad(\text { dex })\end{array}$ & $\begin{array}{l}\text { mode sim } \\
\quad(\text { dex })\end{array}$ & $\begin{array}{c}\sigma_{o b s} \\
(\operatorname{dex})\end{array}$ & $\begin{array}{c}\sigma_{\operatorname{sim}} \\
(\mathrm{dex})\end{array}$ & $\begin{array}{c}1 \\
\left(^{\circ}\right)\end{array}$ & $\begin{array}{c}\mathrm{b} \\
\left(^{\circ}\right)\end{array}$ \\
\hline 000400010000 & -0.42 & -0.539 & -0.315 & -0.706 & 0.51 & 0.553 & 97 & -61 \\
\hline 000400470000 & -0.239 & -0.676 & 0.077 & -0.893 & 0.584 & 0.48 & 325 & -68 \\
\hline 002000010000 & -0.39 & -0.63 & -0.464 & -0.446 & 0.382 & 0.482 & 105 & -63 \\
\hline 002000470000 & -0.255 & -0.718 & -0.139 & -0.517 & 0.49 & 0.709 & 319 & -69 \\
\hline 002959033000 & -0.519 & -0.531 & -0.446 & -0.352 & 0.607 & 0.478 & 110 & -66 \\
\hline 002959500600 & -0.428 & -0.553 & -0.426 & -0.531 & 0.581 & 0.504 & 311 & -67 \\
\hline 003240440056 & -0.264 & -0.493 & -0.111 & -0.279 & 0.447 & 0.425 & 314 & -73 \\
\hline 004000010000 & -0.425 & -0.534 & -0.361 & -0.195 & 0.317 & 0.671 & 117 & -64 \\
\hline 004000470000 & -0.236 & -0.636 & -0.264 & -0.454 & 0.545 & 0.42 & 309 & -70 \\
\hline 005000033000 & -0.316 & -0.479 & -0.104 & -0.526 & 0.363 & 0.389 & 122 & -66 \\
\hline 005000500600 & -0.433 & -0.624 & -0.5 & -0.452 & 0.353 & 0.469 & 304 & -67 \\
\hline 005959010000 & -0.247 & -0.523 & -0.381 & -0.757 & 0.37 & 0.389 & 128 & -64 \\
\hline 005959470000 & -0.483 & -0.608 & -0.527 & -0.248 & 0.491 & 0.568 & 298 & -70 \\
\hline 011959010000 & -0.377 & -0.533 & -0.256 & -0.479 & 0.635 & 0.485 & 139 & -63 \\
\hline 011959470000 & -0.443 & -0.544 & -0.319 & -0.356 & 0.366 & 0.531 & 289 & -69 \\
\hline 014000010000 & -0.375 & -0.526 & -0.461 & -0.568 & 0.466 & 0.498 & 149 & -61 \\
\hline 014000470000 & -0.509 & -0.591 & -0.422 & -0.22 & 0.593 & 0.533 & 280 & -68 \\
\hline 015959010000 & -0.352 & -0.482 & -0.382 & -0.133 & 0.32 & 0.441 & 158 & -59 \\
\hline 015959470000 & -0.508 & -0.489 & -0.394 & -0.325 & 0.505 & 0.466 & 273 & -66 \\
\hline 022000470000 & -0.145 & -0.618 & -0.32 & -0.546 & 0.355 & 0.511 & 268 & -64 \\
\hline 023000033000 & -0.261 & -0.386 & -0.343 & -0.269 & 0.373 & 0.361 & 172 & -57 \\
\hline 023959010000 & -0.304 & -0.54 & -0.196 & -0.448 & 0.528 & 0.426 & 172 & -53 \\
\hline 023959470000 & -0.319 & -0.518 & -0.162 & -0.537 & 0.425 & 0.389 & 263 & -61 \\
\hline 025556002846 & -0.275 & -0.415 & -0.363 & -0.374 & 0.389 & 0.338 & 176 & -50 \\
\hline 025559003000 & -0.234 & -0.305 & -0.3 & -0.186 & 0.231 & 0.271 & 176 & -50 \\
\hline 030000470000 & -0.294 & -0.5 & -0.249 & -0.497 & 0.534 & 0.588 & 260 & -58 \\
\hline 030959500600 & -0.395 & -0.629 & -0.33 & -0.245 & 0.487 & 0.579 & 263 & -55 \\
\hline 031800003000 & -0.388 & -0.484 & -0.266 & -0.182 & 0.627 & 0.453 & 182 & -46 \\
\hline 032000470000 & -0.397 & -0.679 & -0.382 & -0.605 & 0.382 & 0.58 & 257 & -55 \\
\hline 033000500600 & -0.273 & -0.475 & -0.169 & -0.195 & 0.424 & 0.356 & 261 & -52 \\
\hline 033800273000 & -0.39 & -0.693 & -0.289 & -0.651 & 0.475 & 0.485 & 223 & -53 \\
\hline
\end{tabular}


continued from previous page

\begin{tabular}{|c|c|c|c|c|c|c|c|c|}
\hline$\overline{\text { Field }}$ & $\begin{array}{c}\text { mean obs } \\
(\text { dex })\end{array}$ & $\begin{array}{l}\text { mean sim } \\
(\text { dex })\end{array}$ & $\begin{array}{l}\text { mode obs } \\
(\text { dex })\end{array}$ & $\begin{array}{l}\text { mode sim } \\
(\text { dex })\end{array}$ & $\begin{array}{c}\sigma_{o b s} \\
(\mathrm{dex})\end{array}$ & $\begin{array}{c}\sigma_{\text {sim }} \\
(\mathrm{dex})\end{array}$ & $\begin{array}{c}1 \\
\left(^{\circ}\right)\end{array}$ & $\begin{array}{l}\mathrm{b} \\
\left(^{\circ}\right)\end{array}$ \\
\hline 033959000000 & -0.292 & -0.439 & -0.1 & -0.331 & 0.398 & 0.505 & 186 & -41 \\
\hline 033959470000 & -0.337 & -0.457 & -0.324 & -0.236 & 0.376 & 0.461 & 255 & -52 \\
\hline 035959470000 & -0.199 & -0.566 & -0.231 & -0.718 & 0.405 & 0.432 & 254 & -48 \\
\hline 041000500600 & -0.406 & -0.476 & -0.271 & -0.188 & 0.533 & 0.462 & 258 & -46 \\
\hline 041959001959 & -0.292 & -0.512 & -0.326 & -0.196 & 0.361 & 0.46 & 194 & -33 \\
\hline 043000500600 & -0.503 & -0.638 & -0.412 & -0.691 & 0.542 & 0.535 & 257 & -43 \\
\hline 044000500600 & -0.271 & -0.532 & -0.232 & -0.634 & 0.3 & 0.49 & 257 & -41 \\
\hline 050000520000 & -0.375 & -0.551 & -0.164 & -0.24 & 0.454 & 0.491 & 259 & -38 \\
\hline 051959540000 & -0.243 & -0.481 & -0.186 & -0.527 & 0.402 & 0.434 & 262 & -35 \\
\hline 070359423000 & -0.149 & -0.398 & -0.082 & -0.17 & 0.328 & 0.395 & 253 & -16 \\
\hline 072048003000 & -0.228 & -0.302 & -0.053 & -0.15 & 0.303 & 0.23 & 217 & 6 \\
\hline 075600090000 & -0.229 & -0.325 & -0.057 & -0.258 & 0.385 & 0.321 & 228 & 10 \\
\hline 075959003000 & -0.227 & -0.363 & -0.051 & -0.24 & 0.417 & 0.32 & 221 & 15 \\
\hline 082312052959 & -0.251 & -0.42 & -0.143 & -0.165 & 0.337 & 0.396 & 229 & 18 \\
\hline 083959003000 & -0.296 & -0.457 & -0.075 & -0.43 & 0.378 & 0.379 & 227 & 24 \\
\hline 092758003043 & -0.272 & -0.503 & -0.082 & -0.254 & 0.39 & 0.416 & 234 & 34 \\
\hline 092800003000 & -0.313 & -0.493 & -0.097 & -0.236 & 0.437 & 0.465 & 234 & 34 \\
\hline 094753102657 & -0.357 & -0.613 & -0.306 & -0.581 & 0.441 & 0.404 & 247 & 32 \\
\hline 095958410150 & -0.124 & -0.275 & -0.011 & -0.084 & 0.367 & 0.334 & 271 & 11 \\
\hline 100000410000 & -0.129 & -0.335 & -0.018 & -0.032 & 0.316 & 0.436 & 271 & 11 \\
\hline 100759080000 & -0.332 & -0.495 & -0.121 & -0.327 & 0.514 & 0.414 & 249 & 37 \\
\hline 100913412801 & -0.131 & -0.276 & 0.073 & -0.119 & 0.346 & 0.362 & 273 & 12 \\
\hline 101428405235 & -0.145 & -0.295 & 0.015 & -0.102 & 0.297 & 0.373 & 274 & 13 \\
\hline 102800410000 & -0.199 & -0.35 & -0.195 & -0.188 & 0.321 & 0.345 & 276 & 14 \\
\hline 103959410000 & -0.167 & -0.284 & 0.006 & -0.069 & 0.328 & 0.36 & 278 & 15 \\
\hline 105731124726 & -0.585 & -0.569 & -0.285 & -0.319 & 0.598 & 0.401 & 264 & 42 \\
\hline 105808154324 & -0.581 & -0.598 & -0.379 & -0.492 & 0.636 & 0.432 & 267 & 39 \\
\hline 105959410000 & -0.171 & -0.37 & -0.091 & -0.188 & 0.396 & 0.421 & 281 & 17 \\
\hline 110009410232 & -0.181 & -0.318 & 0.035 & -0.174 & 0.422 & 0.363 & 281 & 17 \\
\hline 110053132816 & -0.426 & -0.757 & -0.306 & -0.719 & 0.433 & 0.587 & 266 & 41 \\
\hline 112200100000 & -0.445 & -0.61 & -0.302 & -0.658 & 0.597 & 0.376 & 270 & 47 \\
\hline 112800410000 & -0.126 & -0.372 & -0.057 & -0.173 & 0.435 & 0.402 & 286 & 19 \\
\hline 113159435959 & -0.183 & -0.383 & 0.004 & -0.433 & 0.441 & 0.393 & 288 & 17 \\
\hline 120000090000 & -0.476 & -0.578 & -0.388 & -0.417 & 0.447 & 0.457 & 282 & 52 \\
\hline 120000410000 & -0.213 & -0.477 & -0.177 & -0.213 & 0.441 & 0.418 & 293 & 21 \\
\hline 121159410000 & -0.294 & -0.41 & -0.058 & -0.396 & 0.453 & 0.388 & 295 & 21 \\
\hline 122759410000 & -0.129 & -0.427 & 0.086 & -0.139 & 0.35 & 0.399 & 298 & 22 \\
\hline 124224130559 & -0.451 & -0.582 & -0.205 & -0.362 & 0.472 & 0.398 & 300 & 50 \\
\hline 124359060000 & -0.513 & -0.626 & -0.353 & -0.638 & 0.606 & 0.506 & 300 & 57 \\
\hline 124359090000 & -0.488 & -0.544 & -0.238 & -0.645 & 0.653 & 0.453 & 300 & 54 \\
\hline 124359410000 & -0.124 & -0.317 & -0.124 & -0.149 & 0.403 & 0.37 & 301 & 22 \\
\hline
\end{tabular}


continued from previous page

\begin{tabular}{|c|c|c|c|c|c|c|c|c|}
\hline Field & $\begin{array}{c}\text { mean obs } \\
(\text { dex })\end{array}$ & $\begin{array}{c}\text { mean sim } \\
(\text { dex })\end{array}$ & $\begin{array}{c}\text { mode obs } \\
(\text { dex })\end{array}$ & $\begin{array}{c}\text { mode sim } \\
(\text { dex })\end{array}$ & $\begin{array}{c}\sigma_{o b s} \\
(\operatorname{dex})\end{array}$ & $\begin{array}{c}\sigma_{\text {sim }} \\
(\operatorname{dex})\end{array}$ & $\begin{array}{c}1 \\
\left({ }^{\circ}\right)\end{array}$ & $\begin{array}{c}\mathrm{b} \\
\left(^{\circ}\right)\end{array}$ \\
\hline 125609451238 & -0.254 & -0.324 & -0.211 & -0.131 & 0.396 & 0.396 & 304 & 18 \\
\hline 130047410000 & -0.256 & -0.494 & -0.377 & -0.435 & 0.368 & 0.452 & 305 & 22 \\
\hline 131359410000 & -0.298 & -0.434 & -0.209 & -0.175 & 0.47 & 0.365 & 308 & 22 \\
\hline 131359460007 & -0.136 & -0.385 & -0.19 & -0.386 & 0.351 & 0.423 & 307 & 17 \\
\hline 132000050000 & -0.502 & -0.63 & -0.278 & -0.592 & 0.566 & 0.624 & 316 & 57 \\
\hline 132000090000 & -0.509 & -0.63 & -0.226 & -0.457 & 0.605 & 0.479 & 315 & 53 \\
\hline 132000130000 & -0.331 & -0.519 & -0.326 & -0.395 & 0.486 & 0.514 & 313 & 49 \\
\hline 132800410000 & -0.207 & -0.434 & -0.181 & -0.225 & 0.48 & 0.492 & 310 & 21 \\
\hline 133026434759 & -0.194 & -0.444 & 0.003 & -0.392 & 0.509 & 0.433 & 310 & 19 \\
\hline 134400410000 & -0.214 & -0.444 & -0.309 & -0.245 & 0.375 & 0.411 & 313 & 21 \\
\hline 140000100000 & -0.435 & -0.739 & -0.365 & -0.496 & 0.425 & 0.591 & 329 & 49 \\
\hline 140000410000 & -0.174 & -0.427 & -0.087 & -0.452 & 0.334 & 0.443 & 317 & 20 \\
\hline 140000450000 & -0.177 & -0.368 & -0.156 & -0.309 & 0.283 & 0.373 & 315 & 16 \\
\hline 142000050000 & -0.406 & -0.582 & -0.172 & -0.758 & 0.612 & 0.384 & 340 & 51 \\
\hline 142000410000 & -0.173 & -0.391 & -0.263 & -0.393 & 0.32 & 0.398 & 320 & 19 \\
\hline 142145440827 & -0.179 & -0.449 & -0.169 & -0.371 & 0.374 & 0.426 & 320 & 16 \\
\hline 142800090000 & -0.607 & -0.648 & -0.345 & -0.464 & 0.636 & 0.441 & 339 & 47 \\
\hline 143959410000 & -0.12 & -0.414 & -0.143 & -0.577 & 0.395 & 0.44 & 324 & 17 \\
\hline 144113400831 & -0.215 & -0.477 & -0.154 & -0.237 & 0.402 & 0.488 & 325 & 18 \\
\hline 145800410000 & -0.144 & -0.408 & -0.028 & -0.165 & 0.388 & 0.413 & 327 & 16 \\
\hline 150159100000 & -0.468 & -0.665 & -0.314 & -0.523 & 0.575 & 0.535 & 348 & 41 \\
\hline 150512400000 & -0.297 & -0.395 & -0.343 & -0.142 & 0.438 & 0.487 & 329 & 16 \\
\hline 151111404800 & -0.143 & -0.36 & 0.045 & -0.373 & 0.354 & 0.378 & 330 & 15 \\
\hline 151712413000 & -0.206 & -0.371 & -0.024 & -0.187 & 0.391 & 0.425 & 330 & 14 \\
\hline 152311421200 & -0.179 & -0.297 & -0.285 & -0.003 & 0.398 & 0.406 & 331 & 12 \\
\hline 152936425359 & -0.137 & -0.401 & -0.255 & -0.157 & 0.479 & 0.466 & 331 & 11 \\
\hline 154224441200 & -0.178 & -0.336 & 0.022 & -0.078 & 0.367 & 0.45 & 332 & 9 \\
\hline 155959003000 & -0.503 & -0.65 & -0.457 & -0.36 & 0.552 & 0.544 & 9 & 37 \\
\hline 170024051200 & -0.205 & -0.501 & 0.023 & -0.309 & 0.62 & 0.441 & 15 & 22 \\
\hline 181959470000 & -0.136 & -0.361 & 0.099 & 0.051 & 0.402 & 0.485 & 347 & -14 \\
\hline 200246454536 & -0.522 & -0.629 & -0.438 & -0.575 & 0.495 & 0.469 & 354 & -31 \\
\hline 201959470000 & -0.195 & -0.469 & -0.28 & -0.501 & 0.486 & 0.383 & 353 & -34 \\
\hline 204000471200 & -0.411 & -0.596 & -0.477 & -0.544 & 0.483 & 0.433 & 353 & -38 \\
\hline 211000020000 & -0.233 & -0.551 & -0.257 & -0.417 & 0.34 & 0.508 & 48 & -31 \\
\hline 211959480000 & -0.539 & -0.647 & -0.255 & -0.555 & 0.571 & 0.438 & 351 & -44 \\
\hline 212800020000 & -0.306 & -0.521 & -0.225 & -0.422 & 0.52 & 0.457 & 51 & -35 \\
\hline 215200033000 & -0.414 & -0.616 & -0.226 & -0.62 & 0.539 & 0.521 & 54 & -41 \\
\hline 215959480000 & -0.474 & -0.713 & -0.2 & -0.628 & 0.509 & 0.51 & 349 & -51 \\
\hline 224135480000 & -0.419 & -0.571 & -0.3 & -0.656 & 0.449 & 0.448 & 344 & -57 \\
\hline 230000470000 & -0.198 & -0.516 & -0.215 & -0.391 & 0.467 & 0.432 & 343 & -60 \\
\hline 232000020000 & -0.278 & -0.58 & -0.26 & -0.291 & 0.448 & 0.566 & 78 & -57 \\
\hline
\end{tabular}


continued from previous page

\begin{tabular}{lcccccccc}
\hline \hline Field & $\begin{array}{c}\text { mean obs } \\
(\mathrm{dex})\end{array}$ & $\begin{array}{c}\text { mean sim } \\
(\mathrm{dex})\end{array}$ & $\begin{array}{c}\text { mode obs } \\
(\mathrm{dex})\end{array}$ & $\begin{array}{c}\text { mode sim } \\
(\mathrm{dex})\end{array}$ & $\begin{array}{c}\sigma_{\text {obs }} \\
(\mathrm{dex})\end{array}$ & $\begin{array}{c}\sigma_{\text {sim }} \\
(\mathrm{dex})\end{array}$ & $\begin{array}{c}1 \\
\left({ }^{\circ}\right)\end{array}$ & $\begin{array}{c}\mathrm{b} \\
\left(^{\circ}\right)\end{array}$ \\
\hline 232000480000 & -0.234 & -0.468 & -0.281 & -0.19 & 0.398 & 0.519 & 337 & -63 \\
233000050000 & -0.335 & -0.581 & -0.151 & -0.615 & 0.54 & 0.511 & 78 & -61 \\
234000010000 & -0.298 & -0.568 & -0.374 & -0.565 & 0.513 & 0.422 & 87 & -59 \\
234000470000 & -0.392 & -0.692 & -0.378 & -0.674 & 0.625 & 0.559 & 333 & -66 \\
235000050000 & -0.434 & -0.489 & -0.482 & -0.473 & 0.497 & 0.517 & 87 & -63 \\
235600020000 & -0.442 & -0.437 & -0.41 & -0.511 & 0.448 & 0.342 & 93 & -62 \\
235600480000 & -0.427 & -0.667 & -0.362 & -0.349 & 0.612 & 0.678 & 327 & -66 \\
\hline \hline
\end{tabular}

In order to inspect these results we show in figures 9.15 and 9.16 the field distribution in galactic coordinates color coded by the difference in the means and modes respectively. The figures show that for regions around $l=270^{\circ}$ to $330^{\circ}$ and $b=10^{\circ}$ to $25^{\circ}$ there are fields systematically larger by the same amount even if the dispersion is large. In overall the distribution of the means or modes is positive which means that the observed metallicity distributions are systematically more metal rich than the simulated metallicity distributions.

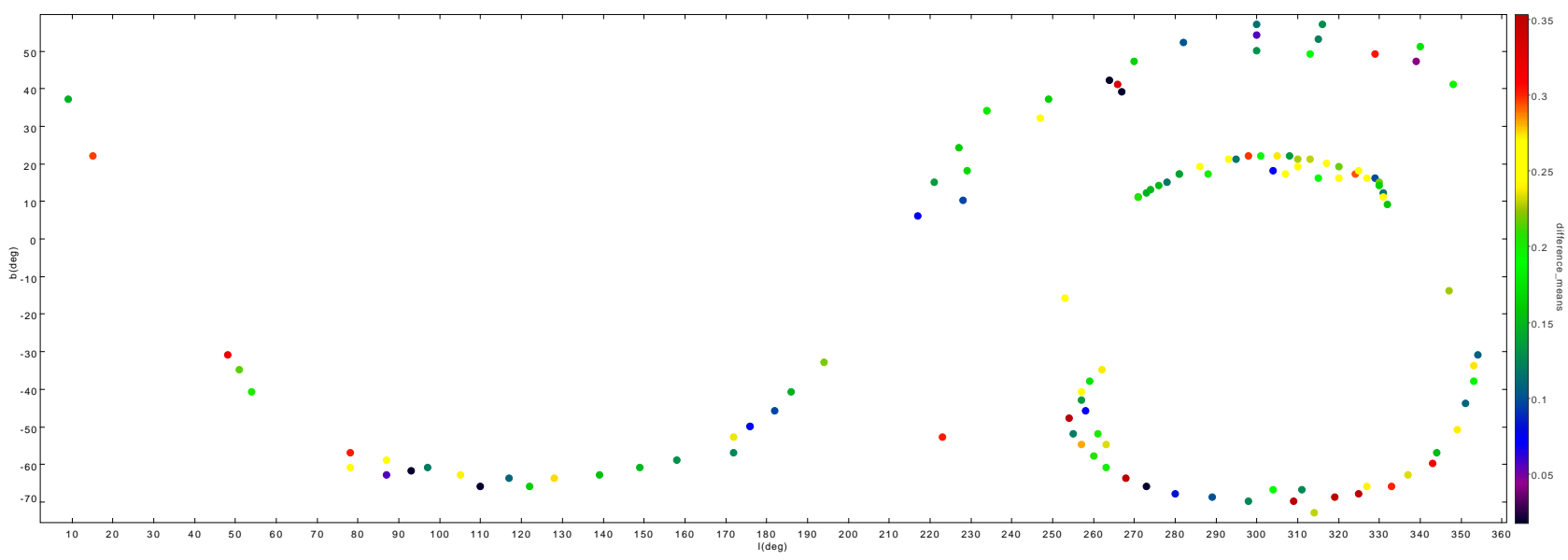

Figure 9.15: Distribution in $(1, b)$ for all fields in GES sample. The color code represents the difference between the mean observation and mean simulation for each field.

\subsubsection{ABC/MCMC analysis for the GES sample}

We used the $A B C / M C M C$ method as explained in section 8.1.5. In this preliminary analysis the fit was done for the three parameters of the young thick disc and the three parameters of the old thin disc. The metallicity distribution of the old thick disc is the one assumed by the model version (i.e. a local value of -0.78 dex, a dispersion of 0.3 and no gradients) (Robin et al. 2014). 


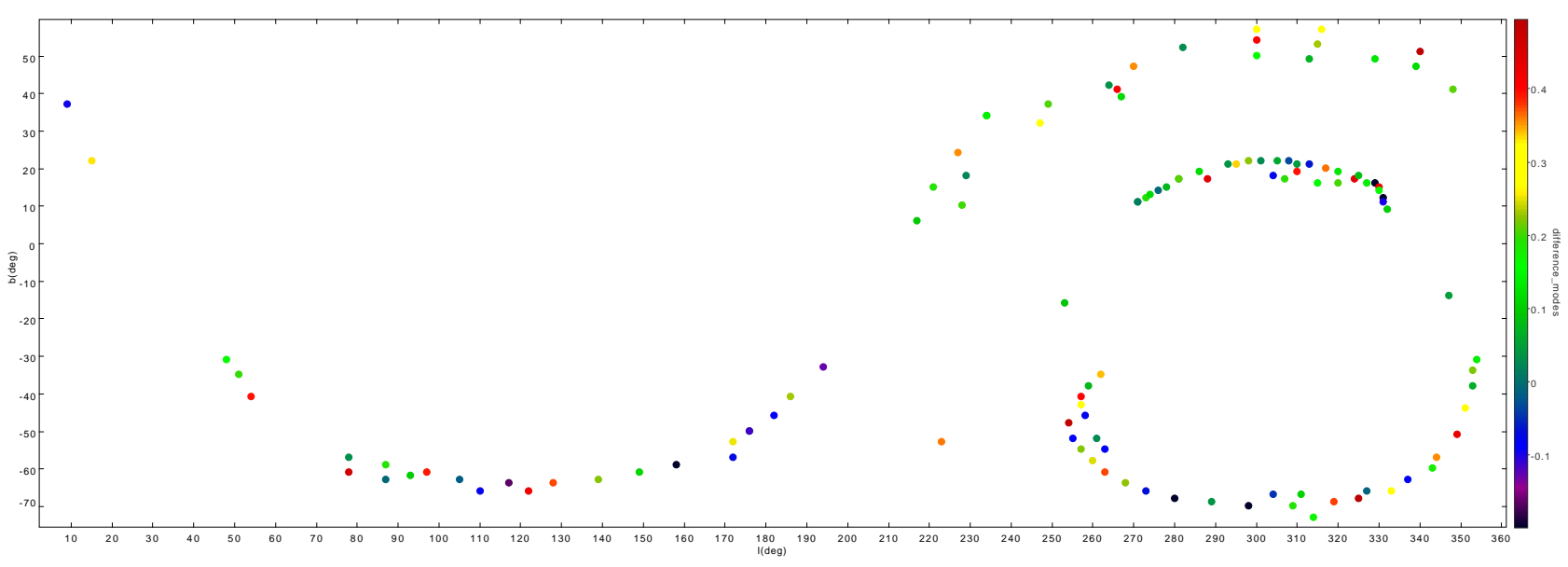

Figure 9.16: Distribution in $(1, b)$ for all fields in GES sample. The color code represents the difference between mode of the observed distribution in metallicity and the mode of the simulated distribution.

Table 9.7:

Set of parameter range. The thick disc parameters have the subscript 'Thick' and refer to the young thick disc. The subscript 'Old Thin' refers to the older ages of the thin disc as explained in section 8.1.5.

\begin{tabular}{lccc}
\hline \hline Parameter & & Minimum & Maximum \\
\hline$\frac{d[F e / H]}{d R}$ & $(d e x / k p c)$ & -0.15 & 0.15 \\
{$[F e / H]_{S N_{\text {Thick }}}$} & $(d e x)$ & -1.0 & -0.1 \\
$(\text { Dispersion })_{\text {Thick }}$ & $(d e x)$ & 0.0 & 0.7 \\
\hline$\frac{d[F e / H]}{d R}$ & $(d e x / k p c)$ & -0.2 & 0.1 \\
{$[\mathrm{Fe} / \mathrm{H}]_{S N_{\text {Old Thin }}}$} & $(d e x)$ & -0.5 & 0.25 \\
$(\text { Dispersion })_{\text {Old Thin }}$ & $($ dex $)$ & 0.0 & 0.6 \\
\hline \hline
\end{tabular}

The parameters range used for this analysis are given in table 9.7. As explained in section 8.1.5 the errors were computed from the final batch of each Markov Chain and by comparison of the different runs for each single simulation. In SEGUE analysis we have performed 10 different simulations and the results of each different simulation were well inside errors given by the $\mathrm{ABC} / \mathrm{MCMC}$ analysis. Therefore, in this preliminary analysis, we assume that the errors due to different simulations are smaller than the ones given by the method but further analysis is needed to confirm it. We have explored the different possible correlations between parameters. In this analysis we used all the above listed fields from GES survey.

Table 9.8 gives the fitted model parameters, along with standard deviation for each parameter. The values are the mean of 10 different $\mathrm{ABC} / \mathrm{MCMC}$ runs which allowed to check if the chain has converged. These results are compatible with a flat radial gradient for the thick disc $\left(0.011 \pm 0.024 \mathrm{dex} \mathrm{kpc}^{-1}\right)$ as in SEGUE analysis but a SN metallicity of $-0.233 \pm 0.038 \mathrm{dex}$ is found which is higher than the one found for SEGUE. The dispersion in the thick disc $(0.173$ 
Table 9.8:

Young thick disc and old thin disc metallicity mean values, when fitting the young thick disc and old thin disc with the GES survey

\begin{tabular}{lccccccc}
\hline \hline $\begin{array}{l}{[\mathrm{Fe} / \mathrm{H}]_{S N_{\text {Thick }}}} \\
(\mathrm{dex})\end{array}$ & $\begin{array}{c}\frac{d[\mathrm{Fe} / \mathrm{H}]}{d R} \\
\left(\mathrm{dex} \mathrm{kpc}^{-1}\right)\end{array}$ & $\begin{array}{c}(\text { Disp })_{\text {Thick }} \\
(\mathrm{dex})\end{array}$ & $\begin{array}{c}{[F e / H]_{S N_{\text {Old Thin }}}} \\
(\mathrm{dex})\end{array}$ & $\begin{array}{c}\frac{d[F e / H]}{d R} \\
\left(\mathrm{dex} \mathrm{kpc}^{-1}\right)\end{array}$ & $\begin{array}{c}(\text { Disp })_{\text {Thin }} \\
(\mathrm{dex})\end{array}$ & $\mathcal{L}$ & $\mathrm{BIC}$ \\
\hline-0.233 & 0.011 & 0.173 & 0.128 & -0.033 & 0.135 & -490.55 & 1033.51 \\
\pm 0.038 & \pm 0.024 & \pm 0.024 & \pm 0.045 & \pm 0.045 & \pm 0.012 & \pm 1.150 & \\
\hline \hline
\end{tabular}

\pm 0.024 dex) is also smaller than the one found with SEGUE analysis.

Regarding the thin disc, results point to a SN metallicity around $0.128 \pm 0.045$ dex which is larger than the one obtained with SEGUE and a dispersion well in agreement with SEGUE data. The radial metallicity gradient is shallower $\left(-0.033 \pm 0.045 \mathrm{dex} \mathrm{kpc}^{-1}\right)$ than the one given by the SEGUE analysis. The dispersion around $0.135 \pm 0.011 \mathrm{dex}$ is well in agreement with the SEGUE analysis and presents no correlation with other parameters.

In figure 9.17 we show the correlations for the ABC/MCMC analysis of the GES sample. The parameters do not show correlations apart of the correlation thick disc local metallicity and gradient. Comparing with SEGUE correlations (Figure 8.8) we noticed that there is no more correlation between the gradient of the old thin disc and the $\mathrm{SN}$ of the old thin disc for the GES sample while the correlation between the gradient and SN metallicity of the thick disc changed sign. The anticorrelation visible in SEGUE results is now a positive correlation with GES sample. We will discuss this result in section 10.2.

\subsection{Discussion}

\subsubsection{The thin disc}

The local metallicity value is still under debate and the errors associated to the measurements from different analysis may be large. The SN metallicity in GES sample is found to be $0.128 \pm 0.045$ dex which is larger than the one obtained from the analysis of SEGUE sample. Both results are well in agreement with the local sample results from Adibekyan et al. (2013) which obtained a value around $-0.06 \pm 0.22$ dex and with figure 9 of Haywood et al. (2013) (which used the same data) but is slightly higher than values around zero as Fuhrmann (2011) which obtained $-0.034 \pm 0.015$ dex from a local sample of stars and Hayden et al. (2014) which obtained $0.02 \pm 0.02$ dex with a APOGEE red giants sample. Other analysis have obtained solar values of $[\mathrm{M} / \mathrm{H}] \sim 0.00$ dex (Haywood 2001, 2002; Taylor \& Croxall 2005; Luck \& Heiter 2006; Fuhrmann 2008; Ivezić et al. 2008; Casagrande et al. 2011). Even lower values, around -0.2 dex, have been obtained (Wyse \& Gilmore 1995; Rocha-Pinto \& Maciel 1996; Soubiran et al. 2003; Nordström et al. 2004; Allende Prieto et al. 2004; Holmberg et al. 2007; Lee et al. 2011). 

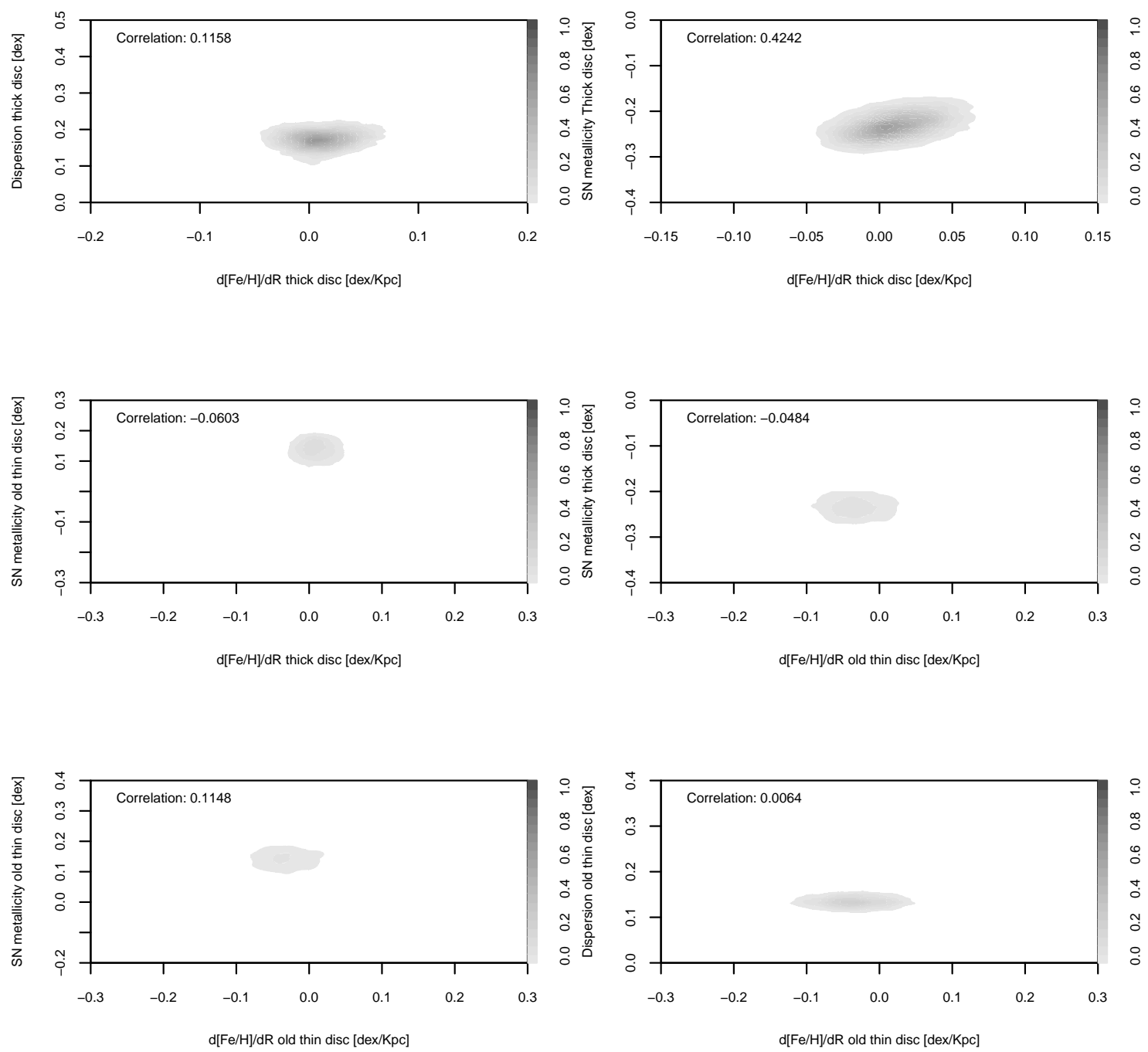

Figure 9.17: Correlations between parameters for the young thick disc and old thin disc fitting for the GES sample analysis. 
Table 8.13 summarizes a selection of literature values.

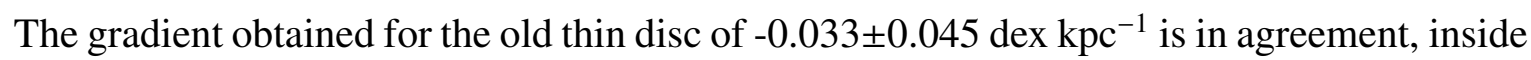
errors, with results obtained from the SEGUE analysis but has a larger error compared with the SEGUE analysis of case 1. Recent literature values from the GES DR1 analysis of $\mathrm{F} / \mathrm{G} / \mathrm{K}$ stars are $-0.058 \pm 0.008 \mathrm{dex} \mathrm{kpc}^{-1}$ (Recio-Blanco et al. 2014) and $-0.044 \pm 0.009 \mathrm{dex} \mathrm{kpc}^{-1}$ (Mikolaitis et al. 2014) which are flatter that the ones obtained from the red giants in APOGEE survey $-0.066 \pm 0.006 \mathrm{dex} \mathrm{kpc}^{-1}$ (Anders et al. 2014) and $-0.09 \pm 0.02 \mathrm{dex} \mathrm{kpc}^{-1}$ (Hayden et al. 2014) and in better agreement with our results. Values for the thin disc gradient are shown in table 8.13.

The dispersion around $0.135 \pm 0.011 \mathrm{dex}$ is in agreement with our SEGUE analysis and with determinations from simulations which obtained values around 0.15 dex (Minchev et al. 2013).

\subsubsection{The thick disc}

As for the thin disc the local metallicity is difficult to constrain and different analysis point to different values. The local thick disc metallicity is found to be $-0.233 \pm 0.038$ higher than the one obtained from the SEGUE analysis. The value is higher than the one obtained by Adibekyan et al. (2013) for the chemically selected thick disc sample but in agreement with their sample of thick disc + high $\alpha$ metal rich stars. The results are also in agreement with local sample from the GCS around $-0.25 \pm 0.20$ (Gilmore et al. 1995) and the most recent analysis of APOGEE red giants in the range $0.5<\mathrm{z}<1.0 \mathrm{kpc}$ which obtained $-0.324 \pm 0.06$ (Hayden et al. 2014). Nevertheless in most of the recent analysis the local metallicity measures are around $-0.5 \mathrm{dex}$ (table 8.14) as obtained in SEGUE analysis.

The gradient in the thick disc, as in SEGUE analysis is found to be flat which is in agreement with literature presented in table 8.14 and discussed in section 8.4.2. 


\section{Chapter 10}

\section{Conclusions and perspectives}

We have analysed a photometric sample of the SEGUE survey, and two spectroscopic samples, a sample of MSTO stars from the SEGUE survey and a sample of F/G/K stars from the GES survey. In the following sections we will analyse the implications of our results on the formation and evolution of the thin and thick disc and give future perspectives to the following work.

\subsection{The photometric sample}

We have analysed the SEGUE low latitudes photometric sample and we noticed that in few fields there was a star count difference between simulations and observations. Assuming that the extinction is properly taken into account this can be due to the presence of a structure or the an indication that the warp slope or the warp model used should be revised.

The preliminary analysis done to the proper motions shown that there is a shift and a larger dispersion in simulations than in observations. The larger dispersion can be due to overestimations of errors or that the velocity dispersion implementation should be revised. The new implementation could take into account smaller age bins and new age-velocity dispersions, even if Gomez et al. (1997) has already been compared with Holmberg et al. (2009) and the former has presented better results (Czekaj et al. 2014).

The analysis done to the revised versions A and B (Czekaj et al. 2014) have shown that model B produces simulations in better agreement with the data.

\subsection{Thin/Thick disc - SEGUE vs GES}

Figures 10.1 and 10.2 show the $\mathrm{R}$ and $\mathrm{Z}$ distribution for the GES and SEGUE samples. The SEGUE sample covers better larger galactocentric distances than the inner galaxy while GES covers better the inner galaxy. The GES sample covers better than SEGUE the vertical distribution. 


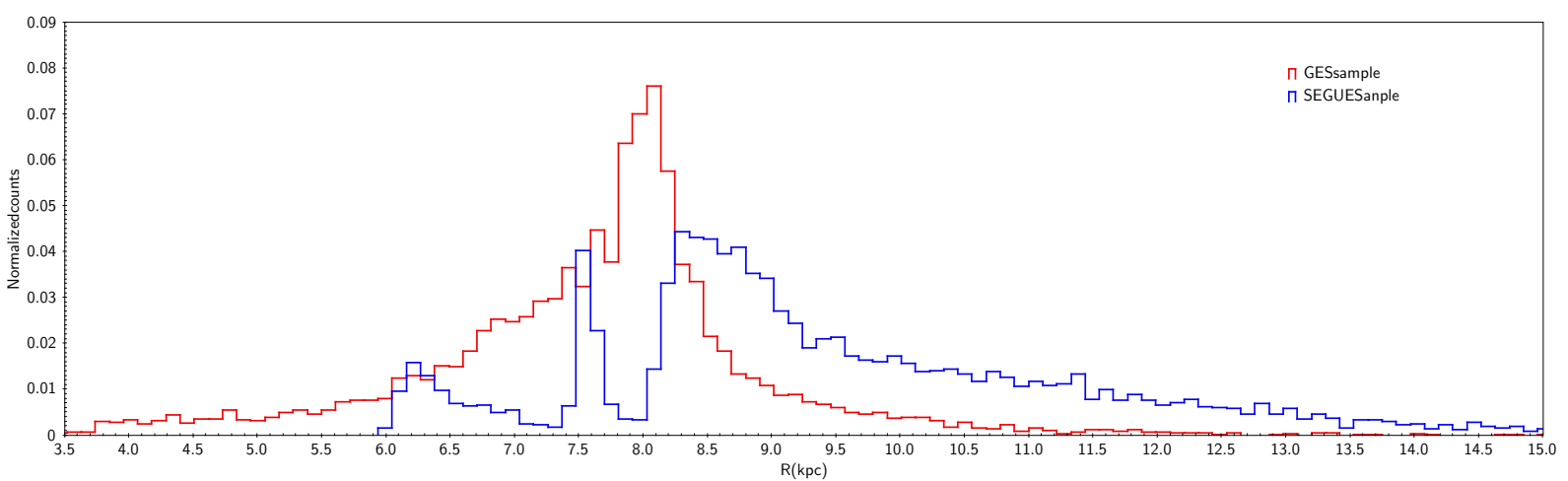

Figure 10.1: Galactocentric radius distribution for the F/G/K stars sample from GES (red line) and MSTO from SEGUE (blue line) as given by the simulations.

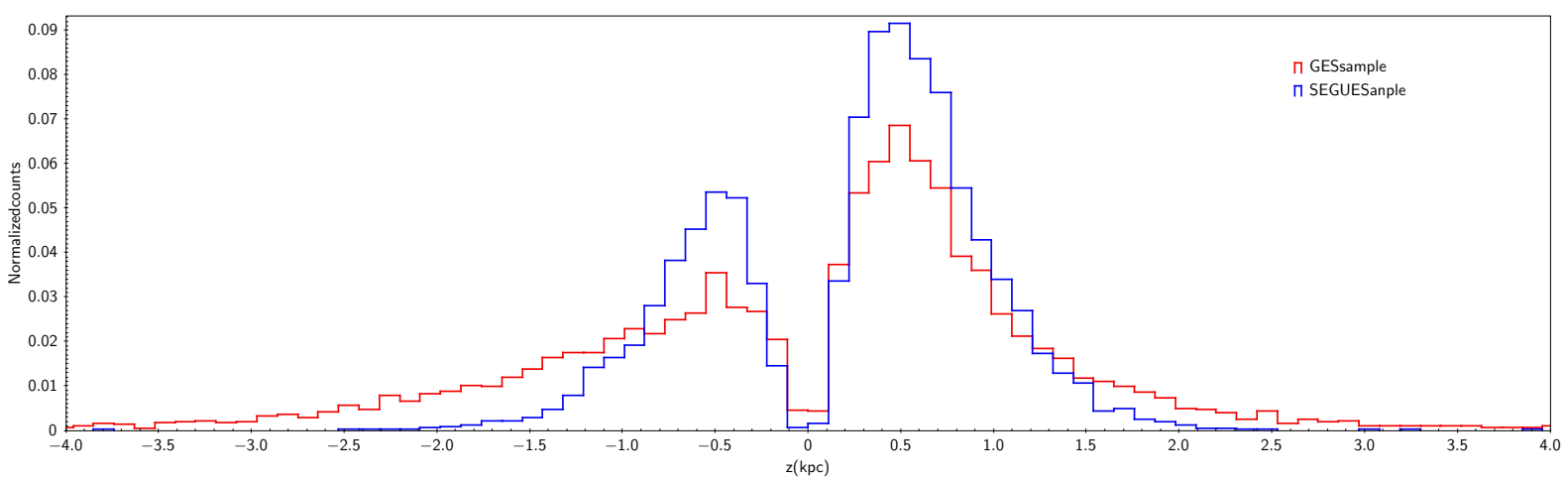

Figure 10.2: Distance from the plane distribution for the $\mathrm{F} / \mathrm{G} / \mathrm{K}$ stars sample from GES (red line) and MSTO from SEGUE (blue line) as given by the simulations. 


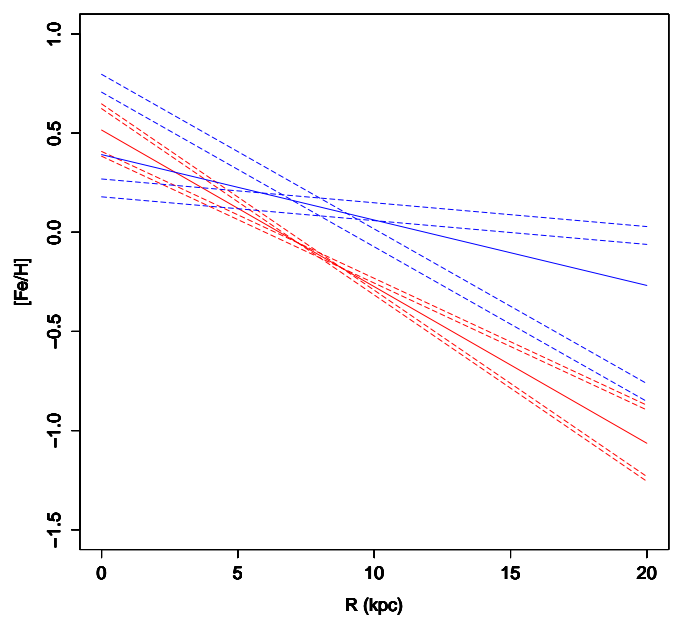

Figure 10.3: Metallicity as a function of the galactocentric radius for the case 1 SEGUE results (red lines) and GES SN metallicity and gradient results for the old thin disc. The continuous lines are the best values obtained and the dashed lines are the combination of the different results taking into account the maximum and minimum values for the errors.

In figure 10.3 we show the range of possible results, in one $\sigma$, of the old thin disc fit for the two different samples. The radial metallicity gradients are compatible but there is a shift in the local value. This shift is in agreement with the GES metallicity distribution in figure 9.11 which peaks at higher metallicities. The use of $\alpha$ element abundances would ease the separation of thin and thick disc samples. In comparison with literature values it is probable that GES sample is slightly shifted towards higher metallicities but further analysis should be done.

Figure 10.4 presents the range of possible results, in one $\sigma$ errors, obtained for the thick disc analysis for SEGUE and GES samples. The radial metallicity gradients are in agreement but as for the thin disc the SN metallicity is higher in the GES sample. As for the thin disc this value seem slightly higher than most of the analysis found in literature. To confirm these results, as for the thin disc, we should make use of abundances in other elements in order to understand better the observational sample and use the information to analyse better possible selection bias.

In SEGUE sample analysis the radial metallicity gradient is anti-correlated with the local metallicity, while in GES sample analysis these parameters are correlated. The SEGUE sample covers better the large galactocentric radius while the GES sample covers better the inner galaxy, as visible in figure 10.1, which means that the correlation between these two parameters is dependent of the mean galactocentric radius of the analysed sample. To make correlations vanish larger number of fields should be use covering more homogeneously the Milky Way. 


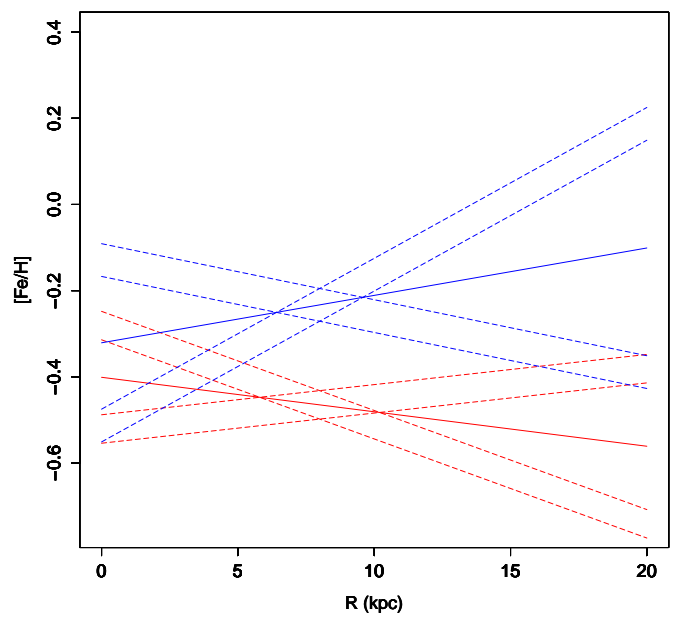

Figure 10.4: Metallicity as a function of the galactocentric radius for the SEGUE results (red lines) and GES SN metallicity and gradient results for the thick disc. The continuous lines are the best values obtained and the dashed lines are the combination of the different results taking into account the maximum and minimum values for the errors.

\subsection{If the thick disc has no radial gradient?}

The indication of a negligible radial gradient, in our best fit, provides some constrains to the thick disc formation and evolution. The thick disc has a SN metallicity around $-0.465 \pm 0.033$ dex and no radial gradient.

The GES preliminary analysis confirms the results obtained with the SEGUE sample of a flat gradient in the thick disc. This result reinforces the indication of a thick disc which formed in an early epoch from a highly turbulent, well mixed gas (scenario (3); see section 2.5.2) producing a chemically homogeneous thick disc (e.g., Brook et al. 2004; Bournaud et al. 2009) or that heavy mixing has occured since this epoch . Radial migration (scenario (5)) can also flatten gradients but Minchev et al. (2011) and Minchev et al. (2012) show that secular radial migration probably has minor effects on building a thick disc. Our results cannot rule out this scenario and it remains open to further discussion. The radial mixing in a disc can be also a consequence of the minor merger bombardment (scenario (2)) (Kazantzidis et al. 2008), (Quillen et al. 2009) and (Bird et al. 2012). In this scenario the radial mixing is stronger at higher $|Z|$ explaining the lack of gradient in the thick disc. The direct accretion of stars (scenario (1)) cannot be ruled out by a null gradient but this scenario predicts orbits with large eccentricities which are not observed in works like Dierickx et al. (2010) from 3D velocities in SDSS and Wilson et al. (2011) from RAVE data. 


\subsubsection{If the thick disc has a positive/negative gradient?}

Our result, from SEGUE analysis, is compatible with a positive gradient in the inner regions

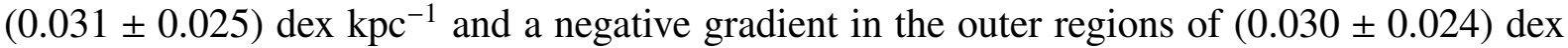
$\mathrm{kpc}^{-1}$. The preliminary GES analysis cannot rule out the gradient inversion but further analysis, such as fitting separately the inner and outer galaxy or testing a two slope gradient in the thick disc as we did in section 8.1.10, are required.

The existence of positive or negative gradients has already been proposed in the literature. Carrell et al. (2012) kinematically selected a sample of F, G and K dwarfs from SDSS DR8 (7.0 $\left.\mathrm{kpc}<R_{\text {gal }}<10.5 \mathrm{kpc}\right)$. The authors sliced the sample in bins of $0.5 \mathrm{kpc}$ in $|Z|$ from $|Z|=1.5$ to $|Z|=3.0$ and obtained radial gradients between $0.025 \pm 0.007 \mathrm{dex} \mathrm{kpc}^{-1}$ and $0.041 \pm 0.016$ dex $\mathrm{kpc}^{-1}$ for the higher distance from the plane (Isochrone Distance Method). Curir et al. (2014) studied the radial metallicity gradient, induced by secular evolution using a N-body simulation to confront their results. The authors find positive gradients of $0.0112 \pm 0.0007 \mathrm{dex}$ $\mathrm{kpc}^{-1}$ for $2.5 \mathrm{kpc}<|Z|<3.0 \mathrm{kpc}$. They conclude that if a positive radial metallicity gradient in the solar neighbourhood is confirmed it is consistent with an early positive radial metallicity gradient in the inner disc $\left(R_{\text {gal }}<10 \mathrm{kpc}\right)$ and a negative in the outer disc $\left(R_{\text {gal }}>10 \mathrm{kpc}\right)$. More recently Hayden et al. (2014) using the APOGEE sample DR10 (1st year of observations) found a gradient inversion using their sample sliced in $0.5 \mathrm{kpc}<|Z|<1.0 \mathrm{kpc}$. The authors found a positive gradient of $0.021 \pm 0.009 \mathrm{dex} \mathrm{kpc}^{-1}$ for inner regions $\left(R_{\text {gal }}<8 \mathrm{kpc}\right)$ and a negative gradient of $-0.053 \pm 0.004 \mathrm{dex} \mathrm{kpc}^{-1}$ for outer regions $\left(R_{\text {gal }}>8 \mathrm{kpc}\right)$. In an inside-out context (Spitoni \& Matteucci (2011), Mott et al. (2013)) the gradient inversion is explained by three different evolution stages. (1) At an early epoch the SFR is higher in the inner regions, but there is a high rate of infalling primordial gas which dilutes more the gas in the inner regions than in outer regions. (2) At later epochs, in the inner regions, the SFR is still strong and the infall of the primordial gas declines which increases the quantity of metals in the interstellar medium. (3) At later epochs the SFR in the inner regions is higher than in the outer regions where there is still a small rate of gas infall. If confirmed, the gradient inversion shows that the direct accretion of stars (scenario (1)) is not the main mechanism that builts the thick disc.

\subsubsection{Perspectives}

In this Phd project we have created a handful number of statistical tools that allowed to analyse large scale surveys and compare it with the BGM which enables a deep study of the structure, formation and evolution of the thick of the Milky Way. In order to constrain the ingredients of the BGM we used two surveys: The SEGUE/SDSS and the GES surveys. We have divided our work in three major parts.

In the first part of the work we analysed the low latitude fields from the SEGUE/SDSS survey. We have devoloped tools to implement correctly the extinction model in the simulations and compare it with observations, by comparing qualitatively and quantitatively, magnitude, color, color-magnitude and proper motions distributions. Even though many work has been 
done to constrain the Milky Way using photometry there are analysis about shape and structure which are worth to do as constrain the warp, flare, disc truncations, scale length among other structures. For this kind of study we could use Red Giant Branch stars as tracers due to their lower dependence of the extinction and because they can reach further distances (Momany et al. 2006).

Further and deeper analysis of proper motions and radial velocities should be done as mentioned before. This analysis are being done by other colleagues who try to improve the model kinematics and compare the model with proper motions and radial velocities.

To further test the revised version (model A and B) (Czekaj et al. 2014) of the BGM larger photometric samples, near the plane, should be used to compare magnitude, color and color magnitude distributions which can give new constraints on the IMF and SFR of each component and SFH in general.

A deeper analysis to the Gaia-ESO sample have to be done to confirm our results which indicate a higher metallicity distribution and a flatter gradient for the thin disc and a higher metallicity distribution for the thick disc compared with literature values and the SEGUE analysis. The same data may be used to confirm a possible inversion of the gradient in the thick disc and constrain the vertical metallicity gradients in thin disc and thick disc, age of the thick disc or even different metallicity distributions for the thin disc components. This data may be used to compute the vertical metallicity gradients in the thin and thick discs as fitting different thin disc gradients according to age. A combination of the SEGUE with Gaia-ESO survey may improve the results of the work and decrease the correlations that exist due to the bias given by using only inner/outer galaxy samples. The analysis of the APOGEE data will provide new constrains on the vertical metallicity gradients and thin disc metallicity distributions because of the high resolution infrared spectroscopy it provides. The combination of large surveys which cover different regions of the galaxy is a step to cover better the space of parameters and possible make disappear possible correlations. The use of kinematical data combined with metallicity distributions would be a further step to understand the thick disc formation. 


\section{Publications}

- André M. M. Martins, Annie C. Robin, Young Sun Lee and Christian P. Robert, Metallicity distribution of the thick disc of the Milky Way from SEGUE low latitude fields Submitted to A\&A

- A. C. Robin, C. Reylé, J. Fliri, M. Czekaj, C. P. Robert, and A. M. M. Martins 2014, A\&A, 569, A13, Constraining the thick disc formation scenario of the Milky Way

- Yun, J. L., Elia, D., Palmeirim, P. M., Gomes, J. I., \& Martins, A. M. 2009, A\&A, 500, 833, Molecular gas and a new young stellar cluster in the far outer Galaxy 


\section{Bibliography}

Abadi, M. G., Navarro, J. F., Steinmetz, M., \& Eke, V. R. 2003, ApJ, 597, 21

Adibekyan, V. Z., Figueira, P., Santos, N. C., et al. 2013, A\&A, 554, A44

Aguerri, J. A. L., Balcells, M., \& Peletier, R. F. 2001, A\&A, 367, 428

Ahn, C. P., Alexandroff, R., Allende Prieto, C., et al. 2014, ApJS, 211, 17

Ahn, C. P., Alexandroff, R., Allende Prieto, C., et al. 2012, ApJS, 203, 21

Aihara, H., Allende Prieto, C., An, D., et al. 2011, ApJS, 193, 29

Alard, C. 2000, ArXiv Astrophysics e-prints

Allard, F. \& Hauschildt, P. H. 1995, ApJ, 445, 433

Allen, C. \& Santillan, A. 1991, 22, 255

Allende Prieto, C., Barklem, P. S., Lambert, D. L., \& Cunha, K. 2004, A\&A, 420, 183

Allende Prieto, C., Sivarani, T., Beers, T. C., et al. 2008, AJ, 136, 2070

Anders, F., Chiappini, C., Santiago, B. X., et al. 2014, A\&A, 564, A115

Arenou, F. 2011, in American Institute of Physics Conference Series, Vol. 1346, American Institute of Physics Conference Series, ed. J. A. Docobo, V. S. Tamazian, \& Y. Y. Balega, $107-121$

Arnett, D. 1996, Space Sci. Rev., 78, 559

Athanassoula, E. 2003, MNRAS, 341, 1179

Aumer, M. \& Binney, J. J. 2009, MNRAS, 397, 1286

Baade, W. 1944, ApJ, 100, 137

Baade, W. 1958, Ricerche Astronomiche, 5, 3

Babusiaux, C., Gómez, A., Hill, V., et al. 2010, A\&A, 519, A77

Bahcall, J. N. \& Soneira, R. M. 1984, ApJS, 55, 67 
Bailin, J. 2003, ApJ, 583, L79

Bailin, J. \& Steinmetz, M. 2003, Ap\&SS, 284, 701

Barbanis, B. \& Woltjer, L. 1967, ApJ, 150, 461

Battaglia, G., Helmi, A., Morrison, H., et al. 2005, MNRAS, 364, 433

Battaglia, G., Helmi, A., Morrison, H., et al. 2006, MNRAS, 370, 1055

Beers, T. C., Carollo, D., Ivezić, Ž., et al. 2012, ApJ, 746, 34

Belokurov, V., Evans, N. W., Irwin, M. J., et al. 2007, ApJ, 658, 337

Bensby, T., Alves-Brito, A., Oey, M. S., Yong, D., \& Meléndez, J. 2011, ApJ, 735, L46

Bensby, T., Feltzing, S., \& Lundström, I. 2003, A\&A, 410, 527

Bensby, T., Feltzing, S., \& Lundström, I. 2004a, Origin and Evolution of the Elements

Bensby, T., Feltzing, S., \& Lundström, I. 2004b, A\&A, 421, 969

Bensby, T., Feltzing, S., \& Lundström, I. 2004c, A\&A, 415, 155

Bensby, T., Zenn, A. R., Oey, M. S., \& Feltzing, S. 2007, ApJ, 663, L13

Bergbusch, P. A. \& Vandenberg, D. A. 1992, ApJS, 81, 163

Bertelli, G., Bressan, A., Chiosi, C., Fagotto, F., \& Nasi, E. 1994, A\&AS, 106, 275

Bertelli, G., Girardi, L., Marigo, P., \& Nasi, E. 2008, A\&A, 484, 815

Bertelli, G., Nasi, E., Girardi, L., \& Marigo, P. 2009, A\&A, 508, 355

Bessell, M. S., Brett, J. M., Wood, P. R., \& Scholz, M. 1989, A\&AS, 77, 1

Bienaymé, O., Robin, A. C., \& Crézé, M. 1987, A\&A, 180, 94

Bilir, S., Karaali, S., Ak, S., Yaz, E., \& Hamzaoğlu, E. 2006, New Astron., 12, 234

Binney, J. \& Tremaine, S. 2008a, Galactic Dynamics: Second Edition (Princeton University Press)

Binney, J. \& Tremaine, S. 2008b, Galactic Dynamics: Second Edition (Princeton University Press)

Bird, J. C., Kazantzidis, S., \& Weinberg, D. H. 2012, MNRAS, 420, 913

Bissantz, N. \& Gerhard, O. 2002, MNRAS, 330, 591

Blumenthal, G. R., Faber, S. M., Primack, J. R., \& Rees, M. J. 1984, Nature, 311, 517

Boeche, C., Siebert, A., Piffl, T., et al. 2013, A\&A, 559, A59 
Boggess, N. W., Mather, J. C., Weiss, R., et al. 1992, ApJ, 397, 420

Bosma, A. 1991, in Warped Disks and Inclined Rings around Galaxies, ed. S. Casertano, P. D. Sackett, \& F. H. Briggs, 181

Boughn, S. \& Crittenden, R. 2004, Nature, 427, 45

Bournaud, F. \& Elmegreen, B. G. 2009, ApJ, 694, L158

Bournaud, F., Elmegreen, B. G., \& Martig, M. 2009, ApJ, 707, L1

Bovy, J. \& Rix, H.-W. 2013, ApJ, 779, 115

Bovy, J., Rix, H.-W., \& Hogg, D. W. 2012a, ApJ, 751, 131

Bovy, J., Rix, H.-W., Liu, C., et al. 2012b, ApJ, 753, 148

Brook, C. B., Governato, F., Roškar, R., et al. 2011, MNRAS, 415, 1051

Brook, C. B., Kawata, D., Gibson, B. K., \& Freeman, K. C. 2004, ApJ, 612, 894

Brunthaler, A., Reid, M. J., Menten, K. M., et al. 2011, Astronomische Nachrichten, 332, 461

Burton, W. B. 1988, The structure of our Galaxy derived from observations of neutral hydrogen, ed. K. I. Kellermann \& G. L. Verschuur, 295-358

Burton, W. B. \& te Lintel Hekkert, P. 1986, A\&AS, 65, 427

Buser, R. 2000, Science, 287, 69

Buser, R., Rong, J., \& Karaali, S. 1999, A\&A, 348, 98

Caldwell, J. A. R. \& Ostriker, J. P. 1981, ApJ, 251, 61

Cardelli, J. A., Clayton, G. C., \& Mathis, J. S. 1989, ApJ, 345, 245

Carlberg, R. G. \& Sellwood, J. A. 1985, ApJ, 292, 79

Carney, B. W., Latham, D. W., \& Laird, J. B. 1990, AJ, 99, 572

Carollo, D., Beers, T. C., Chiba, M., et al. 2010, ApJ, 712, 692

Carollo, D., Beers, T. C., Lee, Y. S., et al. 2007, Nature, 450, 1020

Carollo, D., Freeman, K., Beers, T. C., et al. 2014, ApJ, 788, 180

Carr, B. J. \& Lacey, C. G. 1987, ApJ, 316, 23

Carraro, G. 2014, in IAU Symposium, Vol. 298, IAU Symposium, ed. S. Feltzing, G. Zhao, N. A. Walton, \& P. Whitelock, 7-16

Carraro, G., Beletsky, Y., \& Marconi, G. 2013, MNRAS, 428, 502 
Carrell, K., Chen, Y., \& Zhao, G. 2012, AJ, 144, 185

Carrera, R. \& Pancino, E. 2011, A\&A, 535, A30

Casagrande, L., Schönrich, R., Asplund, M., et al. 2011, A\&A, 530, A138

Castellani, V., Chieffi, A., \& Straniero, O. 1992, ApJS, 78, 517

Catelan, M., Minniti, D., Lucas, P. W., et al. 2011, in RR Lyrae Stars, Metal-Poor Stars, and the Galaxy, ed. A. McWilliam, 145

Cayrel, R., Hill, V., Beers, T. C., et al. 2001, Nature, 409, 691

Chabrier, G. \& Baraffe, I. 1997, A\&A, 327, 1039

Chang, C.-K., Ko, C.-M., \& Peng, T.-H. 2011, ApJ, 740, 34

Chen, B., Figueras, F., Torra, J., et al. 1999, A\&A, 352, 459

Chen, Y. Q., Zhao, G., Carrell, K., \& Zhao, J. K. 2011, AJ, 142, 184

Cheng, J. Y., Rockosi, C. M., Morrison, H. L., et al. 2012a, ApJ, 752, 51

Cheng, J. Y., Rockosi, C. M., Morrison, H. L., et al. 2012b, ApJ, 746, 149

Chiba, M. \& Beers, T. C. 2000, AJ, 119, 2843

Cirasuolo, M., Afonso, J., Bender, R., et al. 2012, in Society of Photo-Optical Instrumentation Engineers (SPIE) Conference Series, Vol. 8446, Society of Photo-Optical Instrumentation Engineers (SPIE) Conference Series

Clewley, L., Warren, S. J., Hewett, P. C., et al. 2005, MNRAS, 362, 349

Combes, F. \& Sanders, R. H. 1981, A\&A, 96, 164

Cox, J. 2008, Astronomy \& Geophysics, 49, 1.7

Crézé, M., Chereul, E., Bienayme, O., \& Pichon, C. 1998, A\&A, 329, 920

Cui, X.-Q., Zhao, Y.-H., Chu, Y.-Q., et al. 2012, Research in Astronomy and Astrophysics, 12, 1197

Curir, A., Serra, A. L., Spagna, A., et al. 2014, ApJ, 784, L24

Czekaj, M. A., Robin, A. C., Figueras, F., Luri, X., \& Haywood, M. 2014, A\&A, 564, A102

Daflon, S., Cunha, K., de la Reza, R., Holtzman, J., \& Chiappini, C. 2010, in IAU Symposium, Vol. 265, IAU Symposium, ed. K. Cunha, M. Spite, \& B. Barbuy, 358-359

Dalton, G., Trager, S. C., Abrams, D. C., et al. 2012, in Society of Photo-Optical Instrumentation Engineers (SPIE) Conference Series, Vol. 8446, Society of Photo-Optical Instrumentation Engineers (SPIE) Conference Series 
Dame, T. M. 1993, in American Institute of Physics Conference Series, Vol. 278, Back to the Galaxy, ed. S. S. Holt \& F. Verter, 267-278

De Angeli, F., Piotto, G., Cassisi, S., et al. 2005, AJ, 130, 116

de Blok, W. J. G. 2010, Advances in Astronomy, 2010

de Jong, J. T. A., Yanny, B., Rix, H.-W., et al. 2010, ApJ, 714, 663

de Jong, R. S., Bellido-Tirado, O., Chiappini, C., et al. 2012, in Society of Photo-Optical Instrumentation Engineers (SPIE) Conference Series, Vol. 8446, Society of Photo-Optical Instrumentation Engineers (SPIE) Conference Series

Debattista, V. P. 2006, in Astronomical Society of the Pacific Conference Series, Vol. 352, New Horizons in Astronomy: Frank N. Bash Symposium, ed. S. J. Kannappan, S. Redfield, J. E. Kessler-Silacci, M. Landriau, \& N. Drory, 161

Debattista, V. P., Mayer, L., Carollo, C. M., et al. 2006, ApJ, 645, 209

Debattista, V. P. \& Sellwood, J. A. 2000, ApJ, 543, 704

Dehnen, W. \& Binney, J. J. 1998, MNRAS, 298, 387

Dekel, A., Sari, R., \& Ceverino, D. 2009, ApJ, 703, 785

Del Popolo, A. 2009, ApJ, 698, 2093

Del Popolo, A., Lima, J. A. S., Fabris, J. C., \& Rodrigues, D. C. 2014, 4, 21

Deng, L.-C., Newberg, H. J., Liu, C., et al. 2012, Research in Astronomy and Astrophysics, 12, 735

Dennis, T. R. 1966, ApJ, 146, 581

Derriere, S. \& Robin, A. C. 2001, in Astronomical Society of the Pacific Conference Series, Vol. 232, The New Era of Wide Field Astronomy, ed. R. Clowes, A. Adamson, \& G. Bromage, 229

Dierickx, M., Klement, R., Rix, H.-W., \& Liu, C. 2010, ApJ, 725, L186

Diplas, A. \& Savage, B. D. 1991, ApJ, 377

Djorgovski, S. \& Sosin, C. 1989, ApJ, 341, L13

Drimmel, R. \& Spergel, D. N. 2001, ApJ, 556, 181

Du, C., Ma, J., Wu, Z., \& Zhou, X. 2006, MNRAS, 372, 1304

Dubinski, J. \& Carlberg, R. G. 1991, ApJ, 378, 496

Dwek, E., Arendt, R. G., Hauser, M. G., et al. 1995, ApJ, 445, 716 
Eggen, O. J., Lynden-Bell, D., \& Sandage, A. R. 1962, ApJ, 136, 748

Eisenstein, D. J., Weinberg, D. H., Agol, E., et al. 2011, AJ, 142, 72

Epchtein, N., Deul, E., Derriere, S., et al. 1999, A\&A, 349, 236

Finkbeiner, A. 2012, Nature, 490, 24

Fluks, M. A., Plez, B., The, P. S., et al. 1994, A\&AS, 105, 311

Flynn, C., Holmberg, J., Portinari, L., Fuchs, B., \& Jahreiß, H. 2006, MNRAS, 372, 1149

Frebel, A., Christlieb, N., Norris, J. E., et al. 2007, ApJ, 660, L117

Freeman, K. \& Bland-Hawthorn, J. 2002, ARA\&A, 40, 487

Freudenreich, H. T. 1998, ApJ, 492, 495

Freudenreich, H. T., Berriman, G. B., Dwek, E., et al. 1994, ApJ, 429, L69

Friel, E. D., Janes, K. A., Tavarez, M., et al. 2002, AJ, 124, 2693

Frinchaboy, P. M., Thompson, B., Jackson, K. M., et al. 2013, ApJ, 777, L1

Fuhrmann, K. 2008, MNRAS, 384, 173

Fuhrmann, K. 2011, MNRAS, 414, 2893

Fux, R. 1997, A\&A, 327, 983

Fux, R. 1999, A\&A, 345, 787

Gardner, E., Debattista, V. P., Robin, A. C., Vásquez, S., \& Zoccali, M. 2014, MNRAS, 438, 3275

Georgelin, Y. M. \& Georgelin, Y. P. 1976, A\&A, 49, 57

Gerhard, O. 2006, in EAS Publications Series, Vol. 20, EAS Publications Series, ed. G. A. Mamon, F. Combes, C. Deffayet, \& B. Fort, 89-96

Gilmore, G., Randich, S., Asplund, M., et al. 2012, The Messenger, 147, 25

Gilmore, G. \& Reid, N. 1983, MNRAS, 202, 1025

Gilmore, G., Wyse, R. F. G., \& Jones, J. B. 1995, AJ, 109, 1095

Girard, T. M., Korchagin, V. I., Casetti-Dinescu, D. I., et al. 2006, AJ, 132, 1768

Girardi, L., Groenewegen, M. A. T., Hatziminaoglou, E., \& da Costa, L. 2005, A\&A, 436, 895

Gomez, A. E., Grenier, S., Udry, S., et al. 1997, in ESA Special Publication, Vol. 402, Hipparcos - Venice '97, ed. R. M. Bonnet, E. Høg, P. L. Bernacca, L. Emiliani, A. Blaauw, C. Turon, J. Kovalevsky, L. Lindegren, H. Hassan, M. Bouffard, B. Strim, D. Heger, M. A. C. Perryman, \& L. Woltjer, 621-624 
Gould, A., Flynn, C., \& Bahcall, J. N. 1998, ApJ, 503, 798

Grabelsky, D. A., Cohen, R. S., Bronfman, L., \& Thaddeus, P. 1988, ApJ, 331, 181

Gunn, J. E., Siegmund, W. A., Mannery, E. J., et al. 2006, AJ, 131, 2332

Gyuk, G., Flynn, C., \& Evans, N. W. 1999, ApJ, 521, 190

Hammersley, P. L., Garzon, F., Mahoney, T., \& Calbet, X. 1995, MNRAS, 273, 206

Hänninen, J. \& Flynn, C. 2002, MNRAS, 337, 731

Hänninen, J. \& Flynn, C. 2004, A\&A, 421, 1001

Hansen, B. M. S., Kalirai, J. S., Anderson, J., et al. 2013, Nature, 500, 51

Hauser, M. G., Kelsall, T., Moseley, Jr., S. H., et al. 1991, in American Institute of Physics Conference Series, Vol. 222, After the first three minutes, ed. S. S. Holt, C. L. Bennett, \& V. Trimble, $161-178$

Hayden, M. R., Holtzman, J. A., Bovy, J., et al. 2014, AJ, 147, 116

Haywood, M. 2001, MNRAS, 325, 1365

Haywood, M. 2002, MNRAS, 337, 151

Haywood, M. 2006a, MNRAS, 371, 1760

Haywood, M. 2006b, MNRAS, 371, 1760

Haywood, M. 2008, MNRAS, 388, 1175

Haywood, M., Di Matteo, P., Lehnert, M. D., Katz, D., \& Gómez, A. 2013, A\&A, 560, A109

Haywood, M., Robin, A. C., \& Crézé, M. 1997a, A\&A, 320, 428

Haywood, M., Robin, A. C., \& Crézé, M. 1997b, A\&A, 320, 440

Helmi, A. 2008, A\&A Rev., 15, 145

Henderson, A. P., Jackson, P. D., \& Kerr, F. J. 1982, ApJ, 263, 116

Henry, R. B. C., Kwitter, K. B., Jaskot, A. E., et al. 2010, ApJ, 724, 748

Hill, V., Lecureur, A., Gómez, A., et al. 2011, A\&A, 534, A80

Holmberg, J., Nordström, B., \& Andersen, J. 2007, A\&A, 475, 519

Holmberg, J., Nordström, B., \& Andersen, J. 2009, A\&A, 501, 941

Ibata, R. A. \& Gilmore, G. F. 1995, MNRAS, 275, 591

Ibukiyama, A. \& Arimoto, N. 2002, A\&A, 394, 927 
Immeli, A., Samland, M., Gerhard, O., \& Westera, P. 2004, A\&A, 413, 547

Ivezić, Ž., Beers, T. C., \& Jurić, M. 2012, ARA\&A, 50, 251

Ivezić, Ž., Sesar, B., Jurić, M., et al. 2008, ApJ, 684, 287

Jahreiß, H. \& Wielen, R. 1997, in ESA Special Publication, Vol. 402, Hipparcos - Venice '97, ed. R. M. Bonnet, E. Høg, P. L. Bernacca, L. Emiliani, A. Blaauw, C. Turon, J. Kovalevsky, L. Lindegren, H. Hassan, M. Bouffard, B. Strim, D. Heger, M. A. C. Perryman, \& L. Woltjer, 675-680

Jenkins, A. 1992, MNRAS, 257, 620

Jenkins, A. \& Binney, J. 1990, MNRAS, 245, 305

Jurić, M., Ivezić, Ž., Brooks, A., et al. 2008, ApJ, 673, 864

Kalirai, J. S. 2012, Nature, 486, 90

Kapteyn, J. C. 1922, ApJ, 55, 302

Kautsch, S. J., Grebel, E. K., Barazza, F. D., \& Gallagher, III, J. S. 2006, A\&A, 445, 765

Kazantzidis, S., Bullock, J. S., Zentner, A. R., Kravtsov, A. V., \& Moustakas, L. A. 2008, ApJ, 688,254

Kendall, M. \& Stuart, A. 1973, The Advanced Theory of Statistics, Vol 2. (Griffin (London)), Ch. 8

Kent, S. M. 1992, ApJ, 387, 181

Kent, S. M., Dame, T. M., \& Fazio, G. 1991, ApJ, 378, 131

Kerr, F. J. \& Lynden-Bell, D. 1986, MNRAS, 221, 1023

Klypin, A., Zhao, H., \& Somerville, R. S. 2002, ApJ, 573, 597

Koch, D., Fazio, G. G., Traub, W. A., et al. 1982, Optical Engineering, 21, 141

Kordopatis, G., Gilmore, G., Steinmetz, M., et al. 2013a, AJ, 146, 134

Kordopatis, G., Gilmore, G., Wyse, R. F. G., et al. 2013b, MNRAS, 436, 3231

Kordopatis, G., Recio-Blanco, A., de Laverny, P., et al. 2011, A\&A, 535, A107

Kormendy, J. \& Fisher, D. B. 2005, in Revista Mexicana de Astronomia y Astrofisica Conference Series, Vol. 23, Revista Mexicana de Astronomia y Astrofisica Conference Series, ed. S. Torres-Peimbert \& G. MacAlpine, 101-108

Kormendy, J. \& Kennicutt, Jr., R. C. 2004, ARA\&A, 42, 603

Kroupa, P. 2002, MNRAS, 330, 707 
Kunder, A., Koch, A., Rich, R. M., et al. 2012, AJ, 143, 57

Kurucz, R. L. 1995, in Astronomical Society of the Pacific Conference Series, Vol. 78, Astrophysical Applications of Powerful New Databases, ed. S. J. Adelman \& W. L. Wiese, 205

Lacey, C. G. \& Ostriker, J. P. 1985, ApJ, 299, 633

Lang, K. R. 2006, A companion to astronomy and astrophysics (Springer Science+Business Media, LLC, )

Laurikainen, E., Salo, H., Athanassoula, E., Bosma, A., \& Herrera-Endoqui, M. 2014, MNRAS, 444, L80

Lee, Y. S., Beers, T. C., An, D., et al. 2011, ApJ, 738, 187

Lee, Y. S., Beers, T. C., Sivarani, T., et al. 2008a, AJ, 136, 2022

Lee, Y. S., Beers, T. C., Sivarani, T., et al. 2008b, AJ, 136, 2050

Lejeune, T., Cuisinier, F., \& Buser, R. 1998, VizieR Online Data Catalog, 413, 65

Lemasle, B., François, P., Genovali, K., et al. 2013, A\&A, 558, A31

Levine, E. S., Blitz, L., \& Heiles, C. 2006, ApJ, 643, 881

Loebman, S. R., Roškar, R., Debattista, V. P., et al. 2011, ApJ, 737, 8

López-Corredoira, M., Cabrera-Lavers, A., Garzón, F., \& Hammersley, P. L. 2002, A\&A, 394, 883

Lopez-Corredoira, M. \& Molgo, J. 2014, ArXiv e-prints

LSST Science Collaboration, Abell, P. A., Allison, J., et al. 2009, ArXiv e-prints

Luck, R. E., Andrievsky, S. M., Kovtyukh, V. V., Gieren, W., \& Graczyk, D. 2011, AJ, 142, 51

Luck, R. E. \& Heiter, U. 2006, AJ, 131, 3069

Maciel, W. J. \& Costa, R. D. D. 2010, in IAU Symposium, Vol. 265, IAU Symposium, ed. K. Cunha, M. Spite, \& B. Barbuy, 317-324

Majewski, S. R., Wilson, J. C., Hearty, F., Schiavon, R. R., \& Skrutskie, M. F. 2010, in IAU Symposium, Vol. 265, IAU Symposium, ed. K. Cunha, M. Spite, \& B. Barbuy, 480-481

Marin, J.-M., Pudlo, P., Robert, C. P., \& Ryder, R. 2011, ArXiv e-prints

Marshall, D. J., Robin, A. C., Reylé, C., Schultheis, M., \& Picaud, S. 2006, A\&A, 453, 635

Martinez-Valpuesta, I. \& Shlosman, I. 2004, ApJ, 613, L29

Mashonkina, L. \& Gehren, T. 2001, A\&A, 376, 232 
Mateo, M. L. 1998, ARA\&A, 36, 435

Matsumoto, T., Hayakawa, S., Koizumi, H., et al. 1982, in American Institute of Physics Conference Series, Vol. 83, The Galactic Center, ed. G. R. Riegler \& R. D. Blandford, 48-52

McMillan, P. J. 2011, MNRAS, 414, 2446

McWilliam, A. \& Zoccali, M. 2010, ApJ, 724, 1491

Melnick, G. J., Fazio, G. G., Koch, D. G., et al. 1987, in American Institute of Physics Conference Series, Vol. 155, The Galactic Center, ed. D. C. Backer, 157-161

Mendez, R. A. \& Guzman, R. 1998, A\&A, 333, 106

Mihalas, D. \& Binney, J. 1981, Galactic astronomy: Structure and kinematics

Mikolaitis, Š., Hill, V., Recio-Blanco, A., et al. 2014, ArXiv e-prints

Minchev, I., Chiappini, C., \& Martig, M. 2013, A\&A, 558, A9

Minchev, I. \& Famaey, B. 2010, ApJ, 722, 112

Minchev, I., Famaey, B., Combes, F., et al. 2011, A\&A, 527, A147

Minchev, I., Famaey, B., Quillen, A. C., et al. 2012, A\&A, 548, A127

Minchev, I. \& Quillen, A. C. 2006, MNRAS, 368, 623

Minniti, D. 1996, ApJ, 459, 175

Minniti, D., Saito, R. K., Alonso-García, J., Lucas, P. W., \& Hempel, M. 2011, ApJ, 733, L43

Minniti, D., White, S. D. M., Olszewski, E. W., \& Hill, J. M. 1992, ApJ, 393, L47

Minniti, D. \& Zoccali, M. 2008, in IAU Symposium, Vol. 245, IAU Symposium, ed. M. Bureau, E. Athanassoula, \& B. Barbuy, 323-332

Momany, Y., Zaggia, S., Gilmore, G., et al. 2006, A\&A, 451, 515

Moore, B., Ghigna, S., Governato, F., et al. 1999, ApJ, 524, L19

Mott, A., Spitoni, E., \& Matteucci, F. 2013, MNRAS, 435, 2918

Munn, J. A., Monet, D. G., Levine, S. E., et al. 2004, AJ, 127, 3034

Nakanishi, H. \& Sofue, Y. 2003, PASJ, 55, 191

Nataf, D. M., Udalski, A., Gould, A., Fouqué, P., \& Stanek, K. Z. 2010, ApJ, 721, L28

Navarro, J. F. \& Benz, W. 1991, ApJ, 380, 320

Navarro, J. F., Frenk, C. S., \& White, S. D. M. 1996, ApJ, 462, 563 
Navarro, J. F., Frenk, C. S., \& White, S. D. M. 1997, ApJ, 490, 493

Navarro, J. F. \& Steinmetz, M. 1997, ApJ, 478, 13

Navarro, J. F. \& Steinmetz, M. 2000, ApJ, 538, 477

Ness, M., Freeman, K., Athanassoula, E., et al. 2013, MNRAS, 430, 836

Neugebauer, G., Habing, H. J., van Duinen, R., et al. 1984, ApJ, 278, L1

Newberg, H. J., Yanny, B., Rockosi, C., et al. 2002, ApJ, 569, 245

Nomoto, K., Iwamoto, K., Nakasato, N., et al. 1997, Nuclear Physics A, 621, 467

Nomoto, K., Yamaoka, H., Shigeyama, T., Kumagai, S., \& Tsujimoto, T. 1994, in Supernovae, ed. S. A. Bludman, R. Mochkovitch, \& J. Zinn-Justin, 199

Nordström, B., Mayor, M., Andersen, J., et al. 2004, A\&A, 418, 989

Ojha, D. K. 2001, MNRAS, 322, 426

Ojha, D. K., Bienaymé, O., Mohan, V., \& Robin, A. C. 1999, A\&A, 351, 945

Ojha, D. K., Bienayme, O., Robin, A. C., Crézé, M., \& Mohan, V. 1996, A\&A, 311, 456

Ojha, D. K., Bienayme, O., Robin, A. C., \& Mohan, V. 1994, A\&A, 290, 771

Ortolani, S., Renzini, A., Gilmozzi, R., et al. 1995, Nature, 377, 701

Perryman, M., Spergel, D. N., \& Lindegren, L. 2014, ApJ, 789, 166

Perryman, M. A. C., de Boer, K. S., Gilmore, G., et al. 2001, A\&A, 369, 339

Perryman, M. A. C., Lindegren, L., Kovalevsky, J., et al. 1997, A\&A, 323, L49

Pettinger, M. M., Bernkopf, J., Fuhrmann, K., Korn, A. J., \& Gehren, T. 2001, in Astronomische Gesellschaft Meeting Abstracts, Vol. 18, Astronomische Gesellschaft Meeting Abstracts, ed. E. R. Schielicke, 166

Pfenniger, D. \& Norman, C. 1990, ApJ, 363, 391

Pichardo, B., Martos, M., \& Moreno, E. 2004, ApJ, 609, 144

Polido, P., Jablonski, F., \& Lépine, J. R. D. 2013, ApJ, 778, 32

Porcel, C., Battaner, E., \& Jimenez-Vicente, J. 1997, A\&A, 322, 103

Purcell, C. W., Kazantzidis, S., \& Bullock, J. S. 2009, in Astronomical Society of the Pacific Conference Series, Vol. 419, Galaxy Evolution: Emerging Insights and Future Challenges, ed. S. Jogee, I. Marinova, L. Hao, \& G. A. Blanc, 248

Quillen, A. C., Minchev, I., Bland-Hawthorn, J., \& Haywood, M. 2009, MNRAS, 397, 1599 
Quinn, P. J., Hernquist, L., \& Fullagar, D. P. 1993, ApJ, 403, 74

Quireza, C., Rood, R. T., Bania, T. M., Balser, D. S., \& Maciel, W. J. 2006, ApJ, 653, 1226

Raha, N., Sellwood, J. A., James, R. A., \& Kahn, F. D. 1991, Nature, 352, 411

Rattenbury, N. J., Mao, S., Sumi, T., \& Smith, M. C. 2007, MNRAS, 378, 1064

Read, J. I., Lake, G., Agertz, O., \& Debattista, V. P. 2008, MNRAS, 389, 1041

Recio-Blanco, A., de Laverny, P., Kordopatis, G., et al. 2014, ArXiv e-prints

Reid, I. N., Gizis, J. E., \& Hawley, S. L. 2002, AJ, 124, 2721

Reid, M. J. 1993, ARA\&A, 31, 345

Reylé, C., Marshall, D. J., Robin, A. C., \& Schultheis, M. 2009, A\&A, 495, 819

Rich, R. M., Reitzel, D. B., Howard, C. D., \& Zhao, H. 2007, ApJ, 658, L29

Rix, H.-W. \& Bovy, J. 2013, A\&A Rev., 21, 61

Robin, A. \& Crézé, M. 1986, A\&A, 157, 71

Robin, A. C., Crézé, M., \& Mohan, V. 1992, ApJ, 400, L25

Robin, A. C., Haywood, M., Creze, M., Ojha, D. K., \& Bienayme, O. 1996, A\&A, 305, 125

Robin, A. C., Marshall, D. J., Schultheis, M., \& Reylé, C. 2012, A\&A, 538, A106

Robin, A. C., Reylé, C., Derrière, S., \& Picaud, S. 2003, A\&A, 409, 523

Robin, A. C., Reylé, C., Fliri, J., et al. 2014, A\&A, 569, A13

Robin, A. C., Rich, R. M., Aussel, H., et al. 2007, ApJS, 172, 545

Rocha-Pinto, H. J. \& Maciel, W. J. 1996, MNRAS, 279, 447

Roediger, J. C., Courteau, S., Graves, G., \& Schiavon, R. P. 2014, ApJS, 210, 10

Rojas-Arriagada, A., Recio-Blanco, A., Hill, V., et al. 2014, ArXiv e-prints

Rubin, V. C., Ford, W. K. J., \& . Thonnard, N. 1980, ApJ, 238, 471

Rubin, V. C., Thonnard, N., \& Ford, Jr., W. K. 1978, ApJ, 225, L107

Rudolph, A. L., Fich, M., Bell, G. R., et al. 2006, ApJS, 162, 346

Ruphy, S., Robin, A. C., Epchtein, N., et al. 1996, A\&A, 313, L21

Saha, K. \& Gerhard, O. 2013, MNRAS, 430, 2039

Saha, K., Martinez-Valpuesta, I., \& Gerhard, O. 2012, MNRAS, 421, 333 
Saito, R. K., Zoccali, M., McWilliam, A., et al. 2011, AJ, 142, 76

Samland, M. \& Gerhard, O. E. 2003, A\&A, 399, 961

Santiago, B. X., Gilmore, G., \& Elson, R. A. W. 1996, MNRAS, 281, 871

Scannapieco, C. \& Tissera, P. B. 2003, MNRAS, 338, 880

Schaller, G., Schaerer, D., Meynet, G., \& Maeder, A. 1992, A\&AS, 96, 269

Schiavon, R. P., Rose, J. A., Courteau, S., \& MacArthur, L. A. 2005, ApJS, 160, 163

Schlegel, D., White, M., \& Eisenstein, D. 2009, in Astronomy, Vol. 2010, astro2010: The Astronomy and Astrophysics Decadal Survey, 314

Schlegel, D. J., Finkbeiner, D. P., \& Davis, M. 1998, ApJ, 500, 525

Schlesinger, K. J., Johnson, J. A., Rockosi, C. M., et al. 2012, ApJ, 761, 160

Schönrich, R., Asplund, M., \& Casagrande, L. 2011, MNRAS, 415, 3807

Schönrich, R. \& Binney, J. 2009a, MNRAS, 396, 203

Schönrich, R. \& Binney, J. 2009b, MNRAS, 399, 1145

Schultz, C., Oñorbe, J., Abazajian, K. N., \& Bullock, J. S. 2014, MNRAS, 442, 1597

Schultz, G. V. \& Wiemer, W. 1975, A\&A, 43, 133

Schuster, W. J., Moitinho, A., Márquez, A., Parrao, L., \& Covarrubias, E. 2006, A\&A, 445, 939

Schwarz, G. 1978, Ann. Stat., 6, 461

Sellwood, J. A. \& Binney, J. J. 2002, MNRAS, 336, 785

Sellwood, J. A. \& Carlberg, R. G. 1984, ApJ, 282, 61

Sharma, S., Bland-Hawthorn, J., Johnston, K. V., \& Binney, J. 2011, ApJ, 730, 3

Siebert, A., Williams, M. E. K., Siviero, A., et al. 2011, AJ, 141, 187

Siegel, M. H., Majewski, S. R., Reid, I. N., \& Thompson, I. B. 2002, ApJ, 578, 151

Silverberg, R. F., Hauser, M. G., Boggess, N. W., et al. 1993, in Society of Photo-Optical Instrumentation Engineers (SPIE) Conference Series, Vol. 2019, Infrared Spaceborne Remote Sensing, ed. M. S. Scholl, 180-189

Simmerer, J., Sneden, C., Cowan, J. J., et al. 2004, ApJ, 617, 1091

Simmons, B. D., Melvin, T., Lintott, C., et al. 2014, ArXiv e-prints

Skrutskie, M. F., Cutri, R. M., Stiening, R., et al. 2006, AJ, 131, 1163 
Smolinski, J. P., Lee, Y. S., Beers, T. C., et al. 2011, AJ, 141, 89

Sneden, C., McWilliam, A., Preston, G. W., et al. 1996, ApJ, 467, 819

Sofue, Y., Honma, M., \& Omodaka, T. 2009, PASJ, 61, 227

Sommer-Larsen, J., Gelato, S., \& Vedel, H. 1999, ApJ, 519, 501

Soto, M., Barbá, R., Gunthardt, G., et al. 2013, A\&A, 552, A101

Soubiran, C. 1994, in IAU Symposium, Vol. 161, Astronomy from Wide-Field Imaging, ed. H. T. MacGillivray, 435

Soubiran, C., Bienaymé, O., \& Siebert, A. 2003, A\&A, 398, 141

Spagna, A., Lattanzi, M. G., Lasker, B. M., et al. 1996, A\&A, 311, 758

Spergel, D. N., Bean, R., Doré, O., et al. 2007, ApJS, 170, 377

Spitoni, E. \& Matteucci, F. 2011, A\&A, 531, A72

Spitzer, Jr., L. \& Schwarzschild, M. 1951, ApJ

Spitzer, Jr., L. \& Schwarzschild, M. 1953, ApJ, 118, 106

Springel, V., Frenk, C. S., \& White, S. D. M. 2006, Nature, 440, 1137

Springel, V. \& Hernquist, L. 2003, MNRAS, 339, 312

Stanek, K. Z., Mateo, M., Udalski, A., et al. 1994, ApJ, 429, L73

Stanghellini, L., Guerrero, M. A., Cunha, K., Manchado, A., \& Villaver, E. 2006, ApJ, 651, 898

Statler, T. S. 1988, ApJ, 331, 71

Steinmetz, M., Zwitter, T., Siebert, A., et al. 2006, AJ, 132, 1645

Stewart, K. R., Bullock, J. S., Wechsler, R. H., Maller, A. H., \& Zentner, A. R. 2008, ApJ, 683, 597

Stoughton, C., Lupton, R. H., Bernardi, M., et al. 2002, AJ, 123, 485

Sumi, T., Woźniak, P. R., Udalski, A., et al. 2006, ApJ, 636, 240

Taylor, B. J. \& Croxall, K. 2005, MNRAS, 357, 967

Terndrup, D. M., Sadler, E. M., \& Rich, R. M. 1995, AJ, 110, 1774

The Fermi-LAT Collaboration, :, Ackermann, M., et al. 2013, ArXiv e-prints

Toth, G. \& Ostriker, J. P. 1992, ApJ, 389, 5 
Tsikoudi, V. 1980, ApJS, 43, 365

Twarog, B. A. 1980, ApJS, 44, 1

Vallee, J. P. 2014, ArXiv e-prints

Vallée, J. P. 2014, MNRAS, 442, 2993

Vallenari, A., Pasetto, S., Bertelli, G., et al. 2006, A\&A, 451, 125

van den Bosch, F. C. 2001, MNRAS, 327, 1334

van Leeuwen, F. 2007, A\&A, 474, 653

Velazquez, H. \& White, S. D. M. 1999, MNRAS, 304, 254

Veltz, L., Bienaymé, O., Freeman, K. C., et al. 2008, A\&A, 480, 753

Villalobos, Á. \& Helmi, A. 2008, MNRAS, 391, 1806

Wang, Y., Zhao, H., Mao, S., \& Rich, R. M. 2012, MNRAS, 427, 1429

Weinberg, M. D. \& Blitz, L. 2006, ApJ, 641, L33

Weiner, B. J. \& Sellwood, J. A. 1999, ApJ, 524, 112

Westera, P., Lejeune, T., Buser, R., Cuisinier, F., \& Bruzual, G. 2002, A\&A, 381, 524

Wielen, R. 1974a, Highlights of Astronomy, 3, 395

Wielen, R. 1974b, Highlights of Astronomy, 3, 395

Wielen, R. 1977, A\&A, 60, 263

Wilson, M. L., Helmi, A., Morrison, H. L., et al. 2011, MNRAS, 413, 2235

Wyse, R. F. G. \& Gilmore, G. 1995, AJ, 110, 2771

Yanny, B., Rockosi, C., Newberg, H. J., et al. 2009, AJ, 137, 4377

Yong, D., Carney, B. W., \& Friel, E. D. 2012, AJ, 144, 95

York, D. G., Adelman, J., Anderson, Jr., J. E., et al. 2000, AJ, 120, 1579

Zhao, G., Zhao, Y.-H., Chu, Y.-Q., Jing, Y.-P., \& Deng, L.-C. 2012, Research in Astronomy and Astrophysics, 12, 723

Zhao, H. \& Mao, S. 1996, MNRAS, 283, 1197

Zoccali, M. 2010, in IAU Symposium, Vol. 265, IAU Symposium, ed. K. Cunha, M. Spite, \& B. Barbuy, 271-278

Zoccali, M., Hill, V., Lecureur, A., et al. 2008, A\&A, 486, 177 
Zoccali, M., Renzini, A., Ortolani, S., et al. 2003, A\&A, 399, 931

Zucker, D. B., de Silva, G., Freeman, K., Bland-Hawthorn, J., \& Hermes Team. 2012, in Astronomical Society of the Pacific Conference Series, Vol. 458, Galactic Archaeology: NearField Cosmology and the Formation of the Milky Way, ed. W. Aoki, M. Ishigaki, T. Suda, T. Tsujimoto, \& N. Arimoto, 421

Zwitter, T., Siebert, A., Munari, U., et al. 2008, AJ, 136, 421 